Florida International University FIU Digital Commons

\title{
A Digital Signal Processing Approach for Affective Sensing of a Computer User through Pupil Diameter Monitoring
}

Ying Gao

Florida International University, YingCindyGao@gmail.com

DOI: $10.25148 /$ etd.FI09120828

Follow this and additional works at: https://digitalcommons.fiu.edu/etd

\section{Recommended Citation}

Gao, Ying, "A Digital Signal Processing Approach for Affective Sensing of a Computer User through Pupil Diameter Monitoring" (2009). FIU Electronic Theses and Dissertations. 132.

https://digitalcommons.fiu.edu/etd/132 


\title{
FLORIDA INTERNATIONAL UNIVERSITY \\ Miami, Florida
}

\section{A DIGITAL SIGNAL PROCESSING APPROACH FOR AFFECTIVE SENSING OF A COMPUTER USER THROUGH PUPIL DIAMETER MONITORING}

\author{
A dissertation submitted in partial fulfillment of the \\ requirements for the degree of \\ DOCTOR OF PHILOSOPHY \\ in \\ ELECTRICAL ENGINEERING \\ by \\ Ying Gao \\ 2009
}


To: Dean Amir Mirmiran

College of Engineering and Computing

This dissertation, written by Ying Gao, and entitled A Digital Signal Processing Approach for Affective Sensing of a Computer User through Pupil Diameter Monitoring, having been approved in respect to style and intellectual content, is referred to you for judgment.

We have read this dissertation and recommend that it be approved.

Malek Adjouadi

Jean Andrian

Wei-Chiang Lin

Armando Barreto, Major Professor

Date of Defense: June 16, 2009

The dissertation of Ying Gao is approved.

Dean Amir Mirmiran

College of Engineering and Computing

Dean George Walker University Graduate School

Florida International University, 2009 
(C) Copyright 2009 by Ying Gao

All rights reserved. 


\section{DEDICATION}

I dedicate this $\mathrm{Ph}$. D. dissertation to my dear parents and my loving boyfriend, for without their patience, understanding, support, and love, the completion of this endeavor would never have been possible. 


\section{ACKNOWLEDGMENTS}

I would like to thank my major Professor, Dr. Armando Barreto, for his excellent guidance and friendship. The National Science Foundation supported the research described in this dissertation through grants CNS-0520811, HRD-0833093, CNS-0426125, IIS-0308155 and HRD-0317692. I would also like to thank the Dissertation Year

Fellowship and Presidential Enhanced Assistantship from Florida International University for their financial support and encouragement during my four years study. Finally, I would like to thank my committee members Dr. Malek Adjouadi, Dr. Jean Andrian and Dr. Wei-Chiang Lin for their expertise and advice. 


\title{
ABSTRACT OF THE DISSERTATION
}

\section{A DIGITAL SIGNAL PROCESSING APPROACH FOR AFFECTIVE SENSING OF A COMPUTER USER THROUGH PUPIL DIAMETER MONITORING}

\author{
by \\ Ying Gao \\ Florida International University, 2009 \\ Miami, Florida

\section{Professor Armando Barreto, Major Professor}

Recent research has indicated that the pupil diameter (PD) in humans varies with their affective states. However, this signal has not been fully investigated for affective sensing purposes in human-computer interaction systems. This may be due to the dominant separate effect of the pupillary light reflex (PLR), which shrinks the pupil when light intensity increases.

In this dissertation, an adaptive interference canceller (AIC) system using the $\mathrm{H}^{\infty}$ time-varying (HITV) adaptive algorithm was developed to minimize the impact of the PLR on the measured pupil diameter signal. The modified pupil diameter (MPD) signal, obtained from the AIC was expected to reflect primarily the pupillary affective responses (PAR) of the subject. Additional manipulations of the AIC output resulted in a processed MPD (PMPD) signal, from which a classification feature, PMPDmean, was extracted. This feature was used to train and test a support vector machine (SVM), for the identification of stress states in the subject from whom the pupil diameter signal was recorded, achieving an accuracy rate of $77.78 \%$. 
The advantages of affective recognition through the PD signal were verified by comparatively investigating the classification of stress and relaxation states through features derived from the simultaneously recorded galvanic skin response (GSR) and blood volume pulse (BVP) signals, with and without the PD feature. The discriminating potential of each individual feature extracted from GSR, BVP and PD was studied by analysis of its receiver operating characteristic (ROC) curve. The ROC curve found for the PMPDmean feature encompassed the largest area (0.8546) of all the single-feature ROCs investigated.

The encouraging results seen in affective sensing based on pupil diameter monitoring were obtained in spite of intermittent illumination increases purposely introduced during the experiments. Therefore, these results confirmed the benefits of using the AIC implementation with the HITV adaptive algorithm to isolate the PAR and the potential of using PD monitoring to sense the evolving affective states of a computer user. 


\section{TABLE OF CONTENTS}

CHAPTER

PAGE

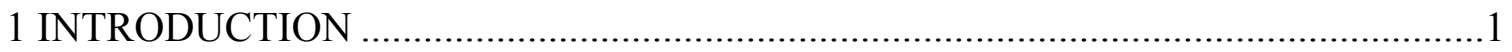

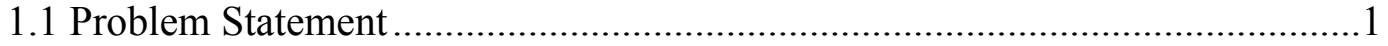

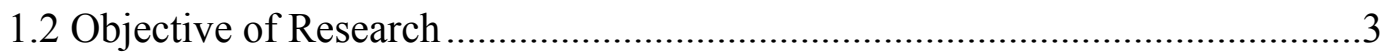

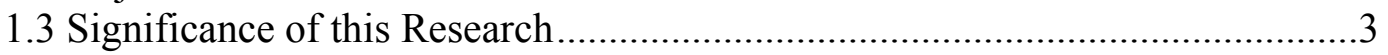

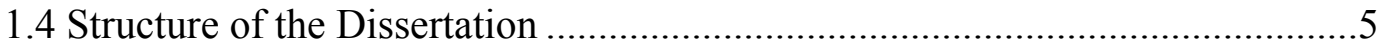

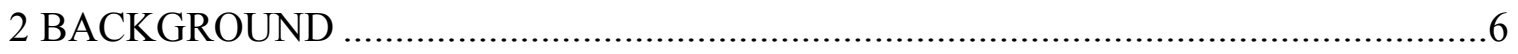

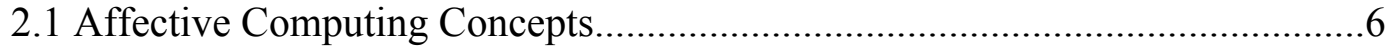

2.2 Autonomic Nervous System ………...........................................................

2.3 Model of Domain Mapping between Psychology and Physiology ...................10

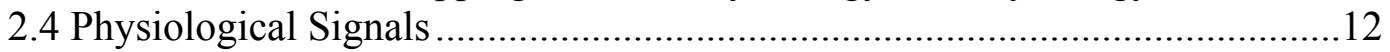

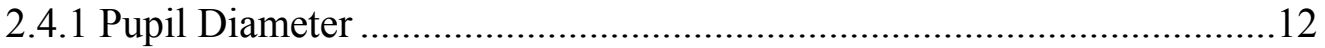

2.4.1.1 Pupillary Light Reflex ...............................................................13

2.4.1.2 Pupillary Affective Response ………………….......................15

2.4.1.3 Pupil Diameter Measurement ....................................................16

2.4.2 Galvanic Skin Response ....................................................................17

2.4.3 Blood Volume Pulse .........................................................................19

2.4.4 Additional Sensing Modalities...........................................................20

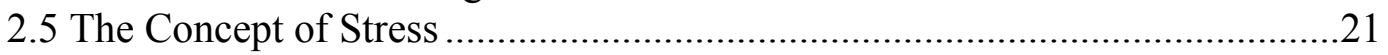

2.6 Previous Experiment Designs for Affective Sensing........................................23

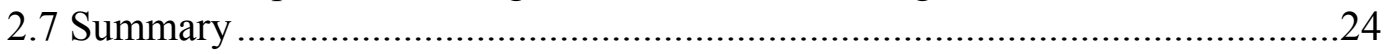

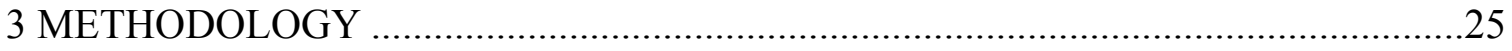

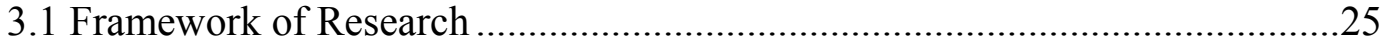

3.2 Adaptive Interference Canceller System …………....................................28

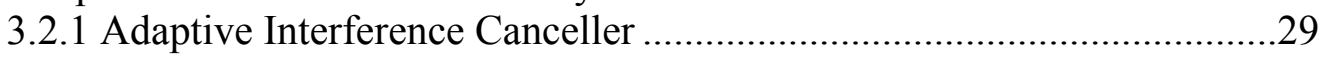

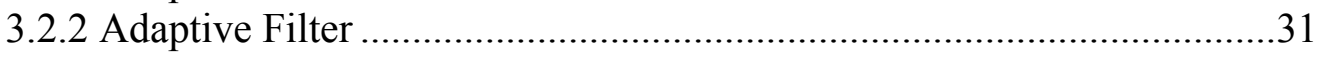

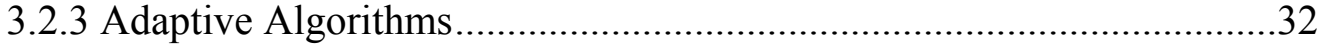

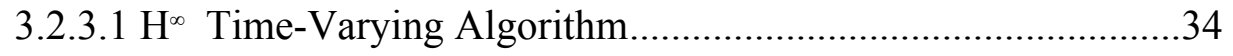

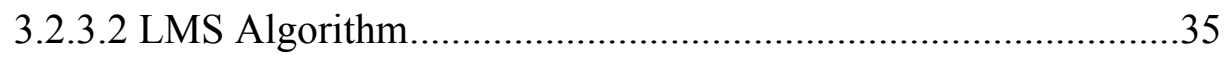

3.2.3.3 Comparison of HITV and LMS Algorithms ................................35

3.3 PD Signal Processing and Feature Extraction Method ....................................40

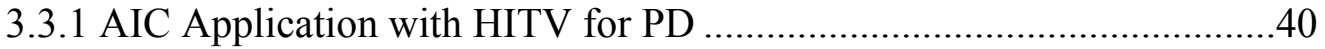

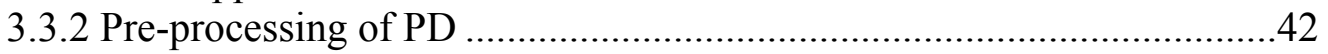

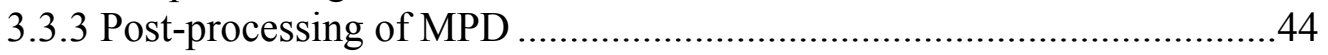

3.3.4 Features Extraction from the PMPD Signal...........................................45

3.4 GSR Signal Processing and Feature Extraction Method ..................................45

3.5 BVP Signal Processing and Feature Extraction Method ....................................4

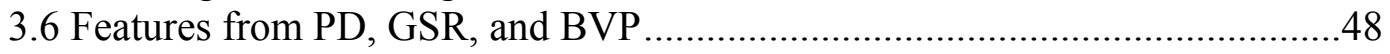

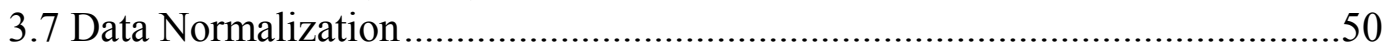

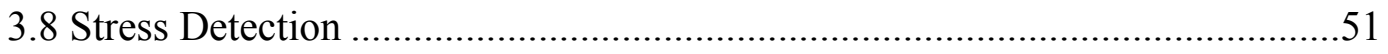


3.8.1 Classification Fundamentals ................................................................51

3.8.2 Support Vector Machine (SVM) .......................................................52

3.8.3 Receiver Operating Characteristic (ROC) …………………..................55

3.9 Overall Research Method Design ..............................................................57

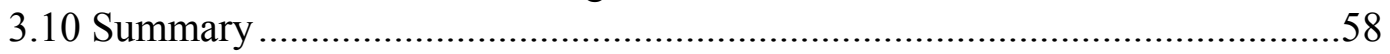

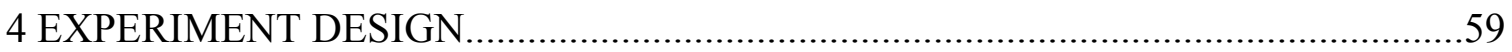

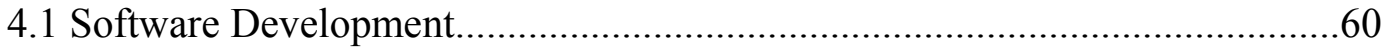

4.1.1 Computer-Based Stroop Test...............................................................60

4.1.2 Stimuli Schedule in Test ..................................................................62

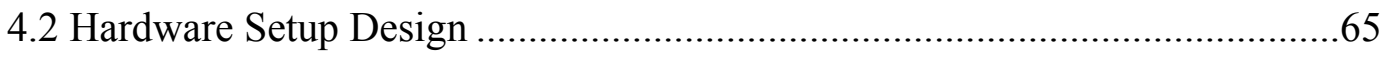

4.2.1 PD Measurement System …………………….................................66

4.2.2 IL Measurement System …………………………...............................68

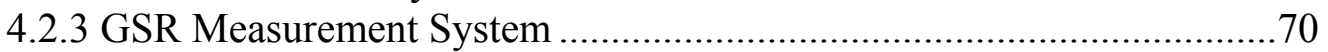

4.2.4 BVP Measurement System ..................................................................72

4.2.5 Synchronization of the Recording of All Signals Measured ...................73

4.2.6 Overall Instrumental Setup .................................................................75

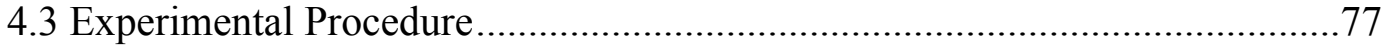

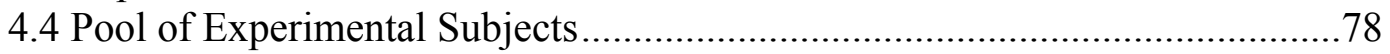

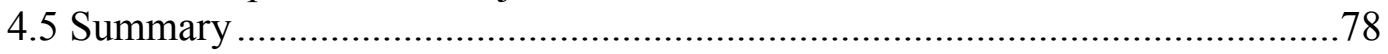

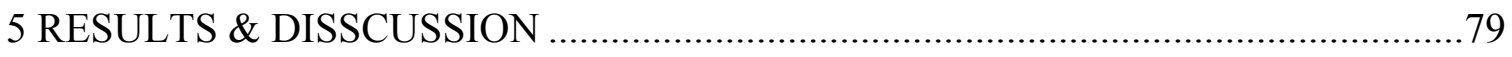

5.1 Signal Processing Results for the PD Signal ................................................79

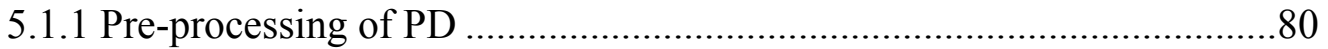

5.1.2 AIC Implementation using the HITV Algorithm.....................................83

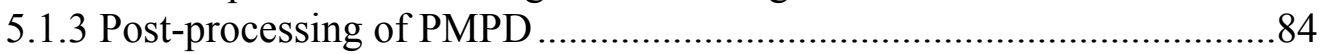

5.1.4 Discussion of PMPD \& PD Signals ........................................................86

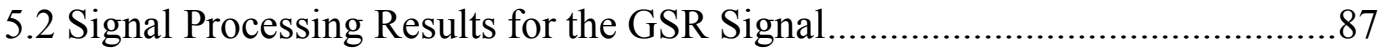

5.3 Signal Processing Results from the BVP Signal............................................89

5.4 Physiological Features Extracted.................................................................93

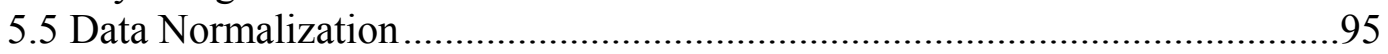

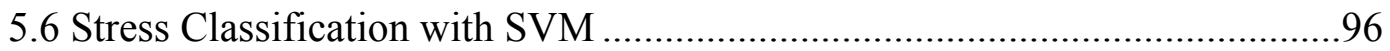

5.7 Discussion of Stress Classification with SVM ..............................................98

5.8 Further Discussion of Affective Sensing through a PD Feature with SVM ....99

5.9 Discussion of Single Feature Discriminating Capability with ROC .............102

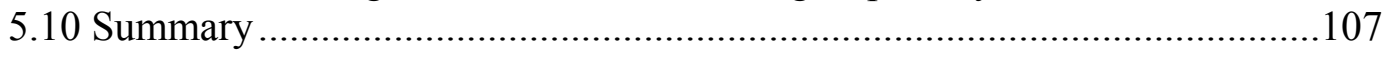

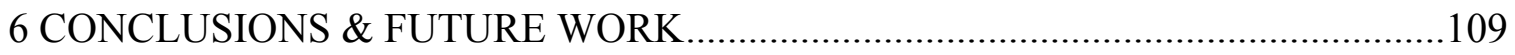

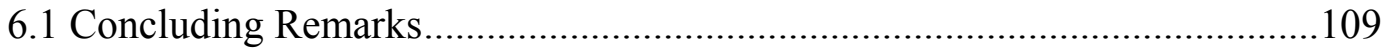

6.2 Limitations of Affective Recognition Approaches Based on Physiological

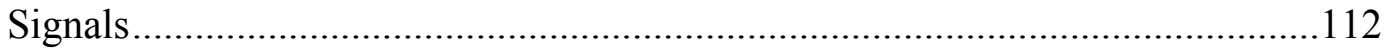

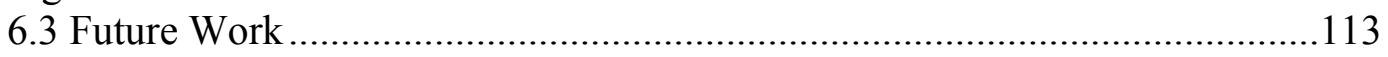

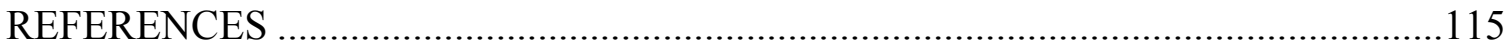

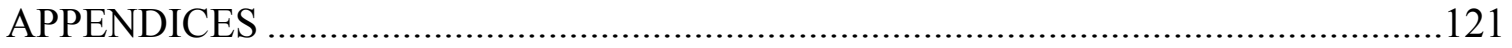

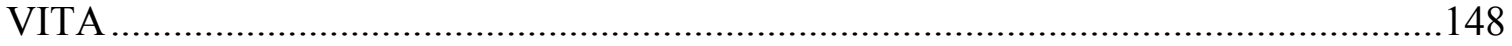




\section{LIST OF TABLES}

TABLE

PAGE

Table 2-1: Functional comparison of SNS and PSNS divisions of the ANS...................10

Table 3-1: Compare of HITV \& LMS adaptive algorithms - AIC results.......................37

Table 3-2: Features extracted from the bio-signals.................................................49

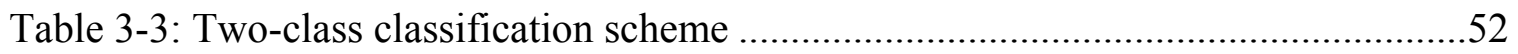

Table 4-1: Possible values of the validity code from the TOBII system .........................68

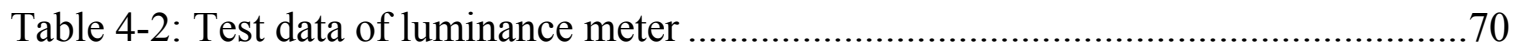

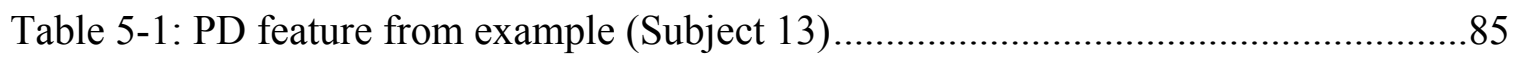

Table 5-2: Five GSR features from example (Subject 13 Segment C2).........................89

Table 5-3: Four BVP features from example (Subject 13 Segment C2) .........................93

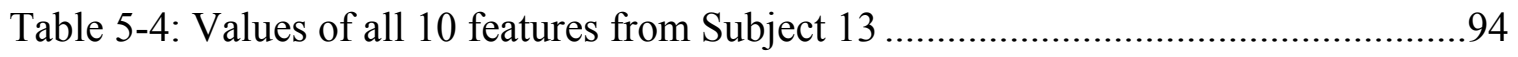

Table 5-5: Results of stress classification by support vector machine ..........................98

Table 5-6: Four groups of PD feature (PMPDmean) for each subject ..........................100

Table 5-7: SVM results for four groups of the PMPDmean feature..............................100

Table 5-8: SVM results for four groups of the PMPDmean and GSRmean features ......101

Table 5-9: SVM results for four groups of the GSRmean feature alone .......................102

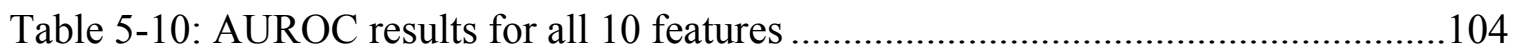




\section{LIST OF FIGURES}

FIGURE

PAGE

Figure 2-1: Affective computing process diagram ..............................................

Figure 2-2: Pathway of the autonomic nervous system .........................................

Figure 2-3: Relationship map of physiological events and psychological processes ........11

Figure 2-4: Functional representation of the PLR system. ........................................ 14

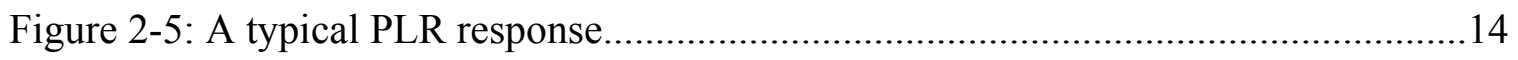

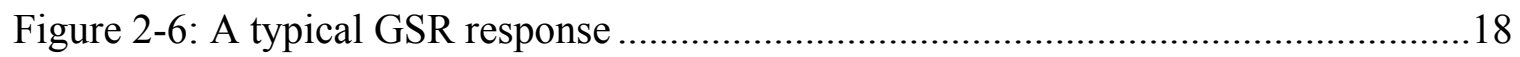

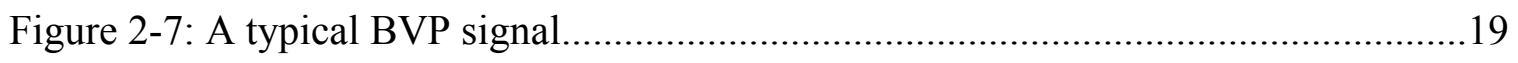

Figure 3-1: Human-computer interaction framework ..............................................26

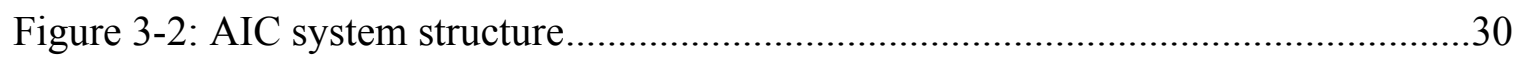

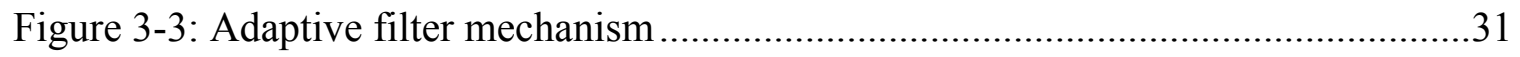

Figure 3-4: Adaptive algorithms compare by PLR system modeling with AIC...............33

Figure 3-5: Adaptive algorithm comparison - blink removal .....................................36

Figure 3-6: Adaptive algorithm comparison - AIC implementation with HITV \& LMS

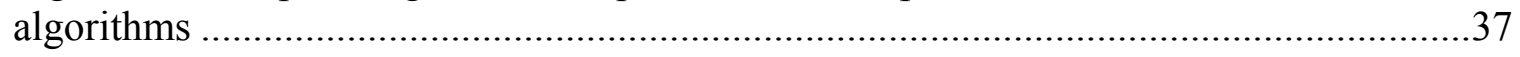

Figure 3-7: Adaptive algorithm comparison - final weights after HITV and LMS

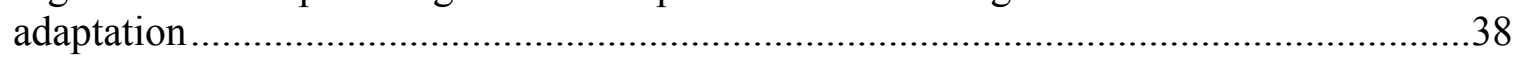

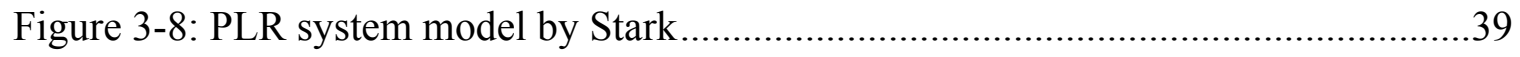

Figure 3-9: PLR system model by Clarke, Zhang and Gamlin.......................................39

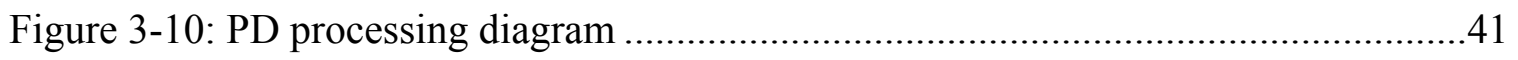

Figure 3-11: HITV parameter RHO changed along with Measured IL ..........................42

Figure 3-12: Frequency response of the FIR filter used for PD pre-processing ...............44

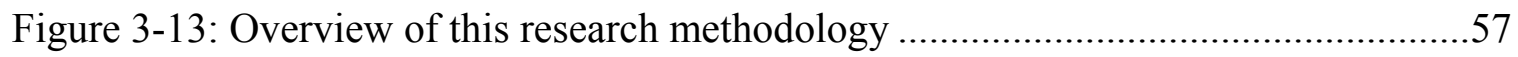


Figure 4-1: Sample Stroop Test interface (Congruent section) ...................................61

Figure 4-2: Sample Stroop Test interface (Incongruent section) ..................................61

Figure 4-3: Sample Stroop Test interface (IC2 with changed button position) .................62

Figure 4-4: Sample Stroop Test interface (IC3 with changed button position) ................62

Figure 4-5: Complete experiment sequence .......................................................63

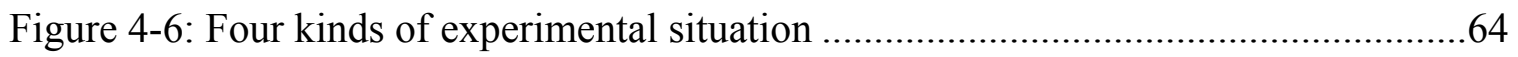

Figure 4-7: DAQ system (PCI-DAS6023 from MCC) .............................................65

Figure 4-8: PD measurement system device - TOBII T60 eye tracker...........................66

Figure 4-9: TOBII T60 eye tracker interface .....................................................67

Figure 4-10: Illumination measurement function ...............................................69

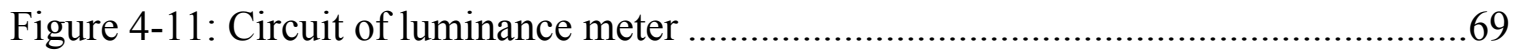

Figure 4-12: Linear relation between luminance and voltage ..................................... 70

Figure 4-13: GSR2 module for GSR measurement system ..................................... 71

Figure 4-14: Frequency to voltage converter circuit for GSR measurement system.........71

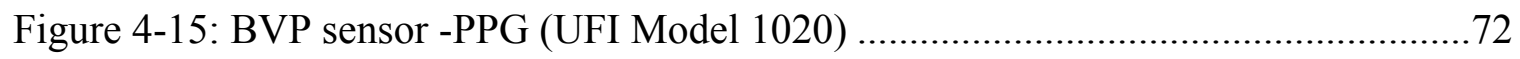

Figure 4-16: The circuit design for the BVP measurement system .............................73

Figure 4-17: Start and Stop button in the Track section of TOBII control program .........74

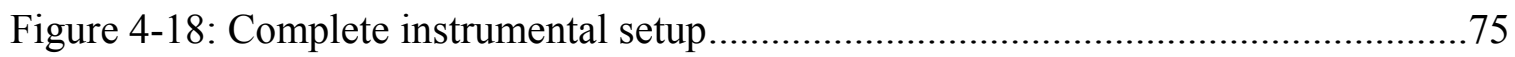

Figure 4-19: An example of participant in the experiment.......................................76

Figure 4-20: PD, IL, GSR and BVP signals in a complete experimental session ............77

Figure 5-1: PD signals and validity codes of both eyes recorded by the TOBII system ...81

Figure 5-2: PD signal before (Measured PD) \& after blink-removal .............................82

Figure 5-3: AIC implementation with HITV algorithm ..........................................84 


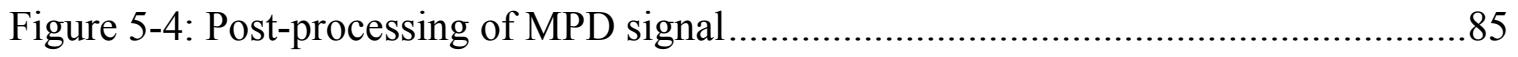

Figure 5-5: Comparison of PMPD with sliding window median analysis on PD .............86

Figure 5-6: Comparison of GSR signal before and after the low pass FIR filter .............87

Figure 5-7: Z-score normalized GSR \& 7-point interpolator application........................88

Figure 5-8: Comparison of BVP signal before and after the low pass FIR filter .............90

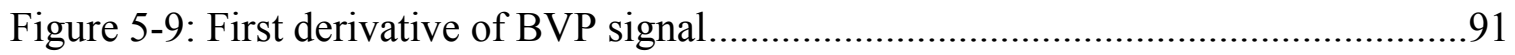

Figure 5-10: Heart beat intervals in samples and milliseconds ..................................92

Figure 5-11: Signal processing results for Subject 13.........................................93

Figure 5-12: The distribution of PMPDmean values for both types of segments ............95

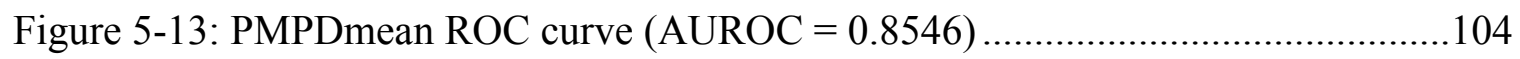

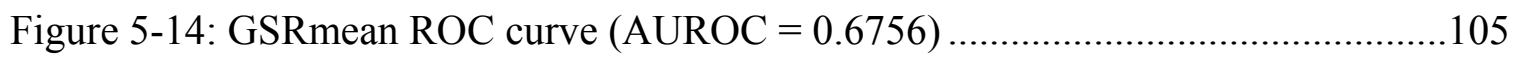

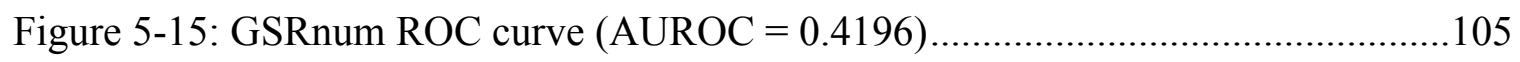

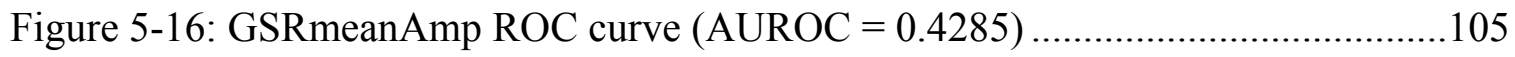

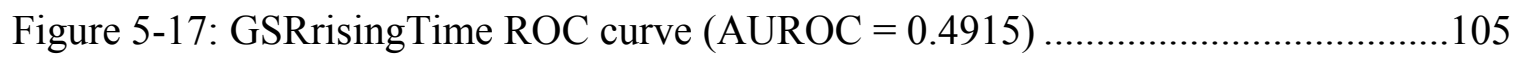

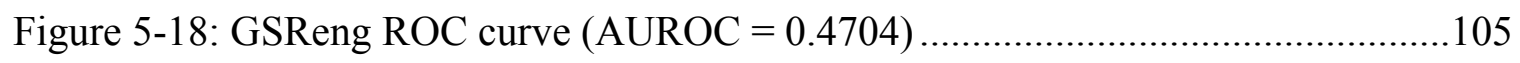

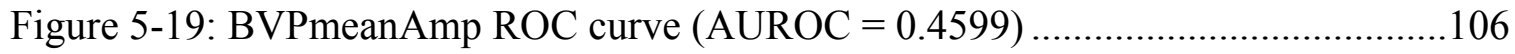

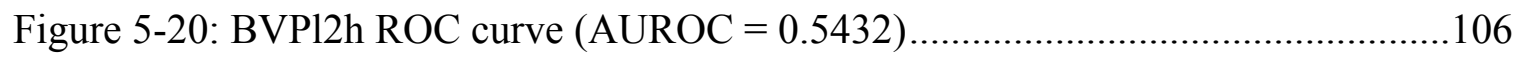

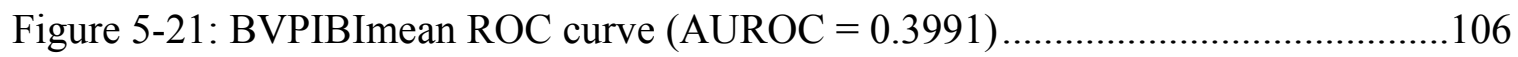

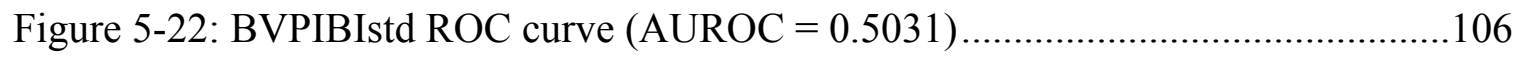

Figure 5-23: Comparison of AUROC values for all 10 features ................................107 


\section{LIST OF SYMBOLS}

SYMBOLS

A:

AccuracyRate The overall success rate for the SVM classifier.

B:

$\mathrm{b}(\mathrm{n})$

BVPmeanAmp The mean value of the amplitude of each BVP beat. (BVP feature)

BVP12h The low frequency to high frequency ratio of the Power Spectral Density of the inter-beat interval (IBI) (the time in milliseconds between two normal, consecutive peaks in the BVP) sequence. (BVP feature)

BVPIBImean The mean value of the IBI sequence. (BVP feature)

BVPIBIstd The standard deviation of the IBI sequence. (BVP feature)

C:

$\mathrm{C}$ The Congruent segment in the Stroop Test, composed of 45 word presentations (font color matches the meaning of the word), which are not expected to elicit significant stress in the subject.

C1 The first Congruent segment in the Stroop Test. (With constant illumination)

C2 The second Congruent segment in the Stroop Test. (With constant illumination) 

illumination)

D: The primary input (measurement) vector of the AIC. (The observed PD signal)

F: The output sequence of the forward difference calculation performed on the BVP signal.

FN False Negative - one of the four outcomes of a classifier. (When the classification result of the classifier is negative, but the actual value is positive)

FP False Positive - one of the four outcomes of a classifier. (When the classification result of the classifier is positive, but the actual value is negative)

G:

The normalized GSR samples for the 7-point Lagrangian interpolation of the GSR signal processing.

$\mathrm{g}^{\prime}[\mathrm{n}] \quad$ The first derivative of $\mathrm{g}(\mathrm{n})$ signal calculated by 3-point Lagrangian interpolation. (The $1^{\text {st }}$ step of the 7-point Lagrangian interpolation of GSR signal processing ) 
The second derivative of $\mathrm{g}(\mathrm{n})$ signal calculated by 7-point Lagrangian interpolation.

ga(k) The gain factor of the HITV adaptive algorithm.

GSRmean The average value of the GSR samples. (GSR feature)

GSRnum The number of the GSR responses. (GSR feature)

GSRmeanAmp The mean value of the amplitude of each GSR response. (GSR feature)

GSRrisingTime The rising time of each GSR response. (GSR feature)

GSReng The energy of GSR (total area under the rising time curve). (GSR feature)

H:

$\mathrm{h}$ The sampling interval in the calculation of the 7-point Lagrangian interpolation.

I:

i

The input (GSR) signal for z-score normalization.

IC The Incongruent segment in the Stroop Test, in which the font color and the meaning of the 30 words presented differ, which is expected to induce stress in the subject.

IC1 The first Incongruent segment in the Stroop Test. (With constant illumination) 

illumination)

IC3 The third Incongruent segment in the Stroop Test. (With constant illumination) The median value in the IL vector.

$\mathrm{IL}_{\text {norm }}$ The normalized IL vector for the calculation of the time-varying parameter $\rho$ in the HITV adaptive algorithm for AIC.

$\mathrm{IL}_{\max }$ The maximum value in the IL vector.

IS The Introductory Segment to let the subject get used to the task environment, in order to establish an appropriate initial level for his/her psychological state, according to the law of initial values.

K:

K The kernel function used in a SVM implementation.

L:

$\mathrm{L}$ The length of AIC input and output signal.

M:

$\mathrm{m}$ The order of the ATF. (Number of weights in the ATF)

N: $\mathrm{n}(\mathrm{k})$ The actual source of the interference for AIC. (The actual IL changes) 
P:

$\mathrm{p}$

PMPDmean The average value of the non-negative PMPD signal. (PD feature)

R:

$\mathrm{r}(\mathrm{k})$

The reference input (interference) vector in the AIC. (The observed IL changes)

RS The resting segment in the Stroop Test to let the subject return to a baseline affective state.

S: The signal of interest in the AIC structure. (PAR)

T: True Negative - one of the four outcomes of a classifier. (When the classification result is negative and the actual value is also negative) TP True Positive - one of the four outcomes of a classifier. (When the classification result is positive and the actual value is also positive)

U: The measurement noise of the reference input of the AIC. (Assumed to be zero in this study) 
V:

$\mathrm{v}(\mathrm{k}) \quad$ The measurement noise vector for the state space model of the ATF. (Including s(k) and model uncertainties $v(\mathrm{k})$ ).

W:

$\mathrm{w}(\mathrm{k}) \quad$ The system state vector for the state space model of the ATF, (The ATF coefficient or weight vector)

$\Delta \mathrm{w}(\mathrm{k}) \quad$ The process noise vector for the state space model of the ATF. (Representing the time variation of the ATF weights)

X:

X

A set of given examples for the SVM classifier.

$\mathrm{X}_{\mathrm{r}} \quad$ The value of a feature during the preliminary period, prior to the effective test. This is used in the data normalization algorithm. (This part of the normalization was done only for GSR and BVP features)

$\mathrm{X}_{\mathrm{s}} \quad$ The raw feature value during relaxation and stress segments in the data normalization algorithm.

Y:

Y

The known labels (targets) used for training a SVM classifier.

$Y_{\text {norm }}$ The output of the last step in the data normalization algorithm. (All features were normalized to the range of $[0,1]$ with max-min normalization) 
$\mathrm{Y}_{\mathrm{s}} \quad$ The output of the first step in the data normalization algorithm. (Features were referenced to their baseline level)

$\mathrm{Y}_{\mathrm{s}}^{\prime} \quad$ The output of the second step in the data normalization algorithm. (Each feature was divided by the sum of all segment values to minimize the impact of individual subject responses).

$\mathrm{Y}_{\mathrm{s} \max }^{\prime}$

$\mathrm{Y}_{\mathrm{s} \min }^{\prime}$

Z:

$\mathrm{Zn}$

$\mathrm{z}(\mathrm{k})$

Others:

$\gamma$

$\varepsilon_{\mathrm{g}}$

$\eta$
The dot product of two vectors.

Convergence rate parameter for LMS adaptive algorithm.

The adjustable parameter of the RBF and Gaussian kernels used for SVM operation.

Parameter for HITV adaptive algorithm. (Positive constant)

Parameter for HITV adaptive algorithm. (Positive constant, reflecting the a priori knowledge of how reliable the initial estimate available for the state vector $\mathrm{w}(0)$ is) 
The mean of the (GSR) signal for z-score normalization.

$\rho$

The time-varying parameter for the HITV adaptive algorithm. (Variable positive constant, reflecting a priori knowledge of how rapidly the state vector $\mathrm{w}(\mathrm{k})$ varies with time).

$\sigma$ The standard deviation of the (GSR) signal for z-score normalization.

$v(\mathrm{k}) \quad$ The model uncertainty vector for the state space model of the ATF. 


\section{CHAPTER 1 \\ INTRODUCTION}

\subsection{Problem Statement}

In the modern world, computers play a role of increasing importance in many areas of society, such as e-Shopping, e-Learning and other everyday tasks. However, the interactions between computers and humans are still limited and mechanistic. Most

commonly, computers react in rigid, prescribed ways to input from humans which, in turn, requires computer users to distort their intuitive interaction patterns, in trying to accommodate the responsive style of the computer. But in today's dynamic social environment and within the current global information era, computers are expected to work more conveniently and efficiently. In order to improve the relationship between human and computers, there seem to be two fundamental alternatives. The first one is to create a computer system that is designed taking into account how the human mind works, using that information to attempt to create a machine matched, from its conception, to that pre-requisite [1]. In contrast, the other alternative is to create a computer system that would not require the user to adapt to the computer, and instead would make the computer tailor its responses differently under different circumstances, aiming to always remain in coordination with the user's emotional state [2].

With regard to the second choice, Picard [2] developed the concept of "affective computing" as "computing that relates to, arises from, or deliberately influences emotions", in 1997. Affective computing pursues the exploration of the techniques, which may lead to the development of a computer-adapting system with the ability to 
observe and understand human emotion. In this context, affective sensing, i.e., the assessment of the user's affective state by the computer, has been considered as one of the key challenges to overcome for improvement of the relationship between human and computers.

Over the last decade, many researchers have carried out studies addressing this affective sensing challenge, such as the attempts at emotion identification through facial expression [3, 4], body gesture [5, 6], and speech processing [7-9] in isolation or in combinations $[10,11]$. Among the diverse affective sensing approaches, the monitoring and analysis of physiological signals is considered as a particularly promising method for affective assessment, since these signals are inherently controlled by the subject's autonomic nervous system (ANS), which means they are less susceptible to environmental interference or voluntary masking [2].

There are several physiological signals that can be chosen for affective state monitoring. Along with other physiological parameters such as the blood volume pulse (BVP), the galvanic skin response (GSR), the skin temperature response (ST), the electromyogram (EMG), etc., the pupil diameter (PD) has been found to be a physiological variable that changes with the subject's mental and emotional state. However, it has not been fully investigated in its potential towards a solution to the affective sensing challenge, which may be due to the well-known confounding effect that the "pupillary light reflex" or "PLR" (i.e., the pupil diameter changes in response to light intensity variation of the environment), could have. 


\subsection{Objective of Research}

This dissertation attempts to explore a way to visualize and evaluate the emotional state identified as "stress" of computer users, through non-invasive and non-obtrusive PD monitoring. An adaptive filtering system is developed and implemented with a selected adaptation algorithm aiming at the minimization of the impact of the PLR on the measured pupil diameter of a computer user, with the hope that, the resulting "modified pupil diameter" (MPD), further enhanced by additional post-processing (PMPD), can be used as an indicator of the PD changes derived solely from the affective response of the subject, which has been termed the pupillary affective response (PAR).

The success of the proposed adaptive filtering system is verified by the individual classification performance of features extracted from the PMPD signal. On the other hand, the stress detection results obtained through features derived from the GSR and BVP with and without the PMPD signal are fully investigated by the implementation and testing of multi-feature classifiers based on the support vector machine (SVM) approach. This comparative study provides an opportunity to determine the advantage of affective sensing through PD vs. other physiological signals (GSR \& BVP). In addition, the individual detection power of each of the 10 features extracted from the signals measured from computer users in this project ( 1 from PD, 5 from GSR and 4 from BVP) is studied through the corresponding receiver operating characteristic (ROC) curve.

\subsection{Significance of this Research}

The pursuit of this research is to explore the techniques which may lead to the development of computer systems enriched with affective awareness. This affective 
recognition will enable the computers to select proper strategies as their responses in the course of human-computer interactions. While this ability could seem somewhat superfluous in some computer systems, it may be critical for the fulfillment of the main purpose of some computer-based systems. For example, it has been clearly established that intelligent tutoring systems (ITS) would benefit from recognizing the emotional state of the learner, as there are emotional conditions that will lead to more efficient learning[12, 13].

In addition, the implementation of affective computing system may contribute to the enhancement of the overall human-computer interaction experience, which may lead to important benefits for the user. For example, working for long periods in front of a computer may cause stress and even fatigue to the computer user. These negative emotions are suspected to be associated with illness and body deterioration. As an example, Jones points out that stressful life events seem to have an association with the onset of breast cancer amongst both the general public and medical professionals [14] . Besides, a large number of studies using different types of methodologies also demonstrated a relationship between stressors and colds, influenza and other infectious diseases $[15,16]$. If the computers are able to become aware of the emergence of this "stress", they could adjust their interaction patterns so as to curb it at an early stage and, therefore, minimize its negative effects.

In summary, enabling computers with the capability of identifying their users' affective states will make it possible for them to respond more appropriately to human emotions, which will greatly enhance the interaction between human and computers. 


\subsection{Structure of the Dissertation}

This proposal is structured in the following manner. Chapter 2 introduces the background information for this dissertation and is concerned with describing the basic properties of some physiological signals and the ways in which other researchers have attempted to use those signals for affective sensing. An overview of affective computing concepts and the emotion model used for this research is also introduced at the beginning of Chapter 2. It covers an outline of the PD, GSR, BVP and other affective monitoring modalities. Chapter 3 states the framework of this research, presents an overview of the adaptive noise-canceling system model, and details the $\mathrm{H}^{\infty}$ time-varying (HITV) adaptive algorithm used for this research. This chapter also includes, the basic ideas associated with the support vector machine (SVM) classifier and receiver operating characteristic (ROC) analysis. Chapter 4 describes the experimental methods followed for this project, from the stress elicitation software to the mechanism of signal acquisition. Chapter 5 presents the PD signal processing results, the total 10 important features extracted from the signals monitored, and the results obtained using machine learning approaches for the recognition of "stress" states from these features. Chapter 5 also includes a discussion of the results obtained. Chapter 6 concludes this dissertation and summarizes further plans suggested for the amplification of this research. 


\section{CHAPTER 2}

\section{BACKGROUND}

This chapter provides the background information for this dissertation topic. It begins with the introduction of the affective computing concepts, followed by the description of the autonomic nervous system (ANS), which controls the physiological response related to a subject's emotional status. The third section outlines the model of emotion used in this research and presents selected examples of prior work in recognizing emotion from physiological changes. This chapter also presents three different types of physiological signals used in this research for the purpose of affective sensing: the pupil diameter (PD), the galvanic skin response (GSR) or electrodermal activity, and the blood volume pulse (BVP). Additionally, a brief introduction to the study of stress and the experimental methods to analyze the stress phenomenon is included. The final section shows some examples of other prior investigations related to experiments designed to detect emotion from physiological signals.

\subsection{Affective Computing Concepts}

Affective computing, one of the emerging areas in the field of human-machine interaction studies, is the exploration of techniques that enable computer systems to sense and adapt to the affective state of their user. According to Picard [2], affective computing involves not only emotion detection, but also extends to the implementation of emotions, and attempts to give the computer the ability to recognize and express emotions. Barreto [17] identified three key components in the affective computing process initially proposed by Hudlicka [18] in 2003: 
(i) Affect Sensing and Recognition;

(ii) User Affect Modeling / Machine Affect Modeling;

(iii) Machine Affect Expression.

The interaction of these key processes involved in an Affective Computing implementation is shown in Figure 2-1.

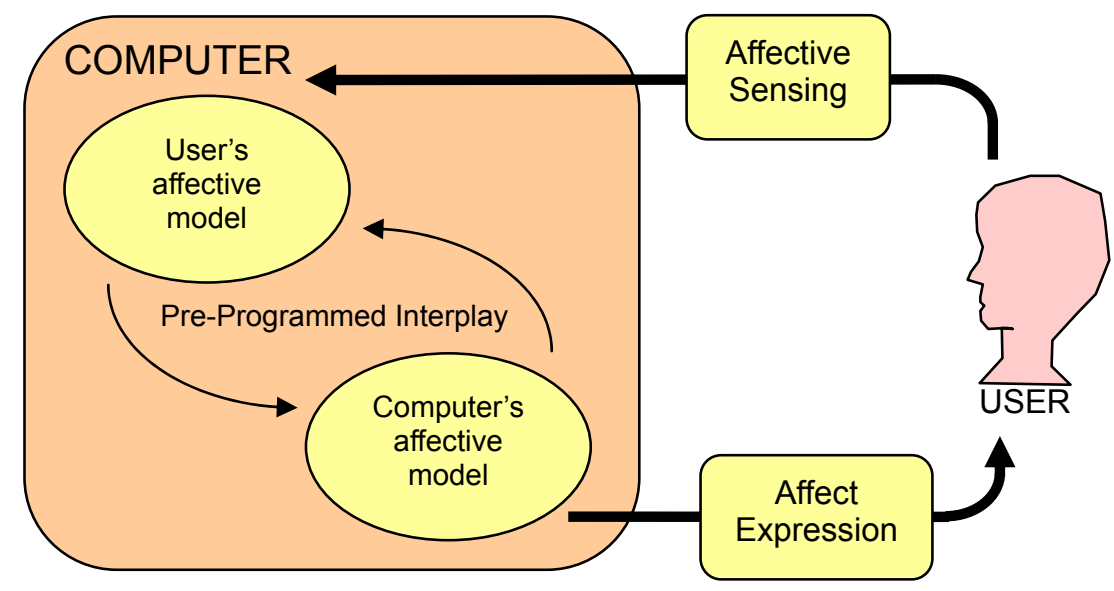

Figure 2-1: Affective computing process diagram [17]

From the figure above, it is clear that affective sensing is a core aspect of affective computing in general. To date, a variety of approaches have been proposed to meet the challenge of affect recognition, including the use of speech processing; facial expressions; body gesture; and psychophysiological measures, etc.. This dissertation focuses on affective reorganization by monitoring of psychophysiological signals, with the advantage that they reflect autonomic nervous system (ANS) reactions and are thus difficult to intentionally distort or supress. Thanks to recent improvements in wearable remote sensors and complex pattern recognition algorithms, the real-time differentiation of user affective states may be applicable to real-world human-machine collaboration in a near future. 


\subsection{Autonomic Nervous System}

Generally, the autonomic nervous system (ANS) is a regulatory structure transmitting impulses from the central nervous system to peripheral organ systems, which helps human adapt to changes in their environment. On the basis of functional differences, the ANS is mainly divided into three separate systems, the sympathetic, parasympathetic, and enteric nervous systems. The enteric nervous system is generally accepted today as a separate system whose main function is to innervate the gut region of the body, although traditionally it was not included as a major division of the ANS[19]. Knowledge about the basic structure and function of the ANS is very important to understand psychophysiological phenomena. The ANS affects organs, such as the heart and the sweat glands, which are the focus of psychophysiology. In addition, the ANS activity mediates stress responses and emotional arousal, which are two core concepts of the mind-body interface as well as many psychosomatic diseases.

The sympathetic nervous system (SNS), as a part of the ANS, originates in the thoracic and lumbar regions of the spinal cord, and regulates many homeostatic mechanisms in the human body. Fibers from the SNS innervate tissues in almost every organ system, providing some regulatory function to phenomena as diverse as pupil diameter, gut motility, and urinary output. When fully activated, this division readies the body for a crisis that may require sudden, intense physical activity, which is commonly known as the "fight-or-flight" response, and corresponds with arousal and energy generation. 
On the other hand, the parasympathetic nervous system (PSNS) of the ANS originates in the brain stem and the lower part of the spinal cord, and typically functions in opposition to the SNS. This division stimulates visceral activity and is associated with the relaxation of the body, which is known as a "rest and digest" response. Its activation promotes a return of several organs to regular function, and enhances digestion.

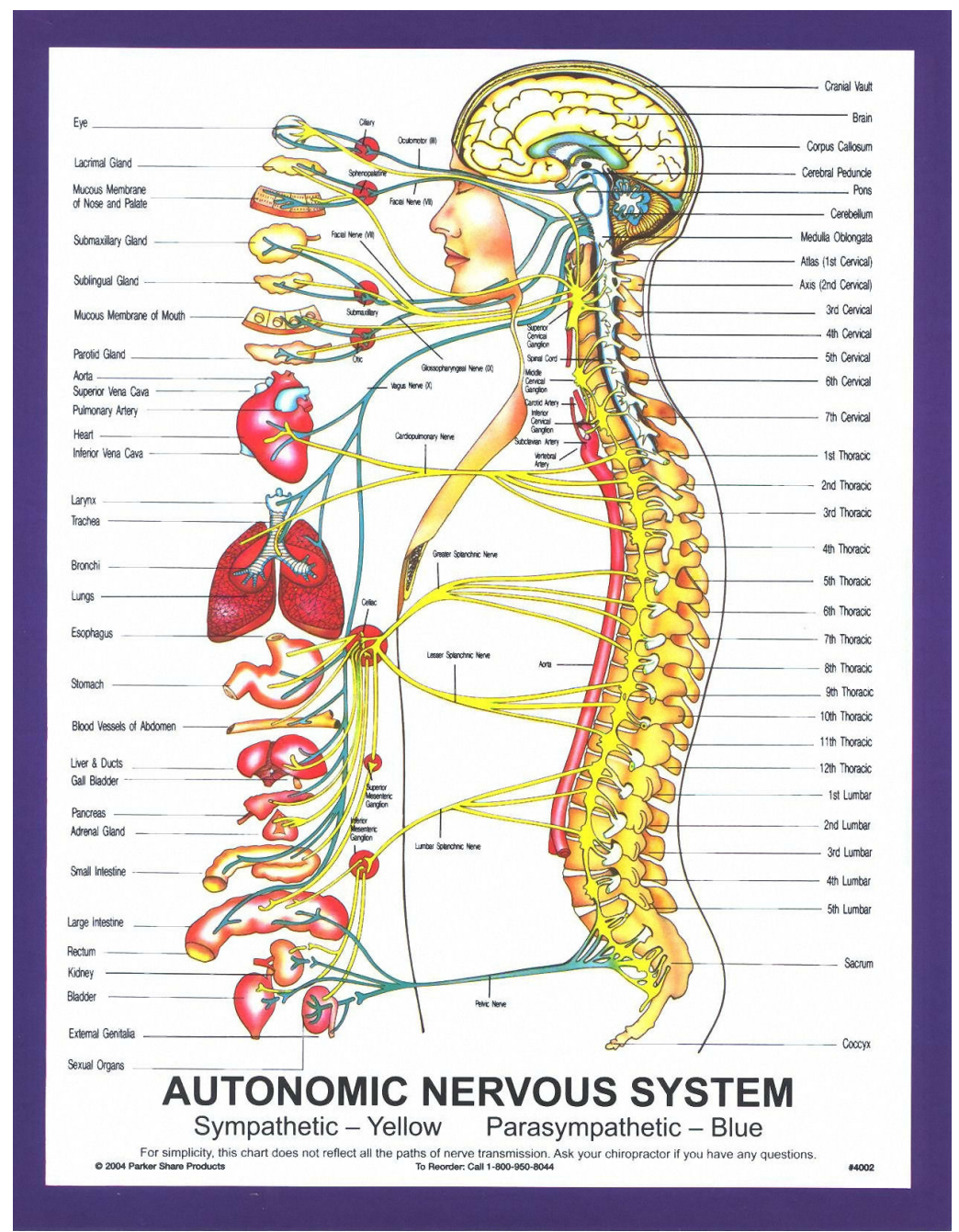

Figure 2-2: Pathway of the autonomic nervous system

The SNS and PSNS systems are shown in the Figure 2-2 above, which may work independently with some structures innervated by only one division or the other. However, they may also work jointly to control a given stage of a complex process. In 
general, the sympathetic division typically functions in actions requiring quick responses such as emergency, stress or exertion. The parasympathetic division functions with actions that do not require immediate reaction (e.g., under resting conditions). A general comparison between these two divisions is summarized in Table 2-1 (from[20]), where $\alpha$ and $\beta$ are two classes of sympathetic receptors.

Table 2-1: Functional comparison of SNS and PSNS divisions of the ANS

\begin{tabular}{lll}
\hline Structure & Sympathetic stimulation & Parasympathetic stimulation \\
\hline Heart & Increased rate & Decreased rate \\
& Increased contractile force & Decreased contractile force \\
Lungs & Bronchodilation & Bronchoconstriction \\
Kidneys & Decreased output & No change \\
$\underline{\text { Systemic blood vessels }}$ & & \\
\multicolumn{1}{c}{ Abdominal } & Constricted & None \\
\multicolumn{1}{c}{ Muscle } & Constricted $(\alpha)$ & None \\
\multicolumn{1}{c}{ Skin } & Dilated $(\beta)$ & None \\
Liver & Constricted & None \\
Blood glucose & Glucose release & Slight glycogen synthetic \\
$\underline{\text { Pupils }}$ & Increased & None \\
$\underline{\text { Sweat glands }}$ & Dilated & Constricted \\
Basal metabolism & Copious sweating & None \\
Skeletal muscle & Increased up to $100 \%$ & None \\
\hline
\end{tabular}

\subsection{Model of Domain Mapping between Psychology and Physiology}

Half a century ago, John Lacey (1959) [21] published an influential review, which pioneered research on the use of psychophysiological approaches to evaluate 
psychotherapeutic outcomes. These studies have been amplified and expanded by more recent investigations. In 1990, Cacioppo and Tassinary [22] proposed a model for the relationship between physiological events and psychological or behavioral processes, which maps physiological variables to an emotion space. The development of such model is essential for the affective recognition through unique ANS patterns.

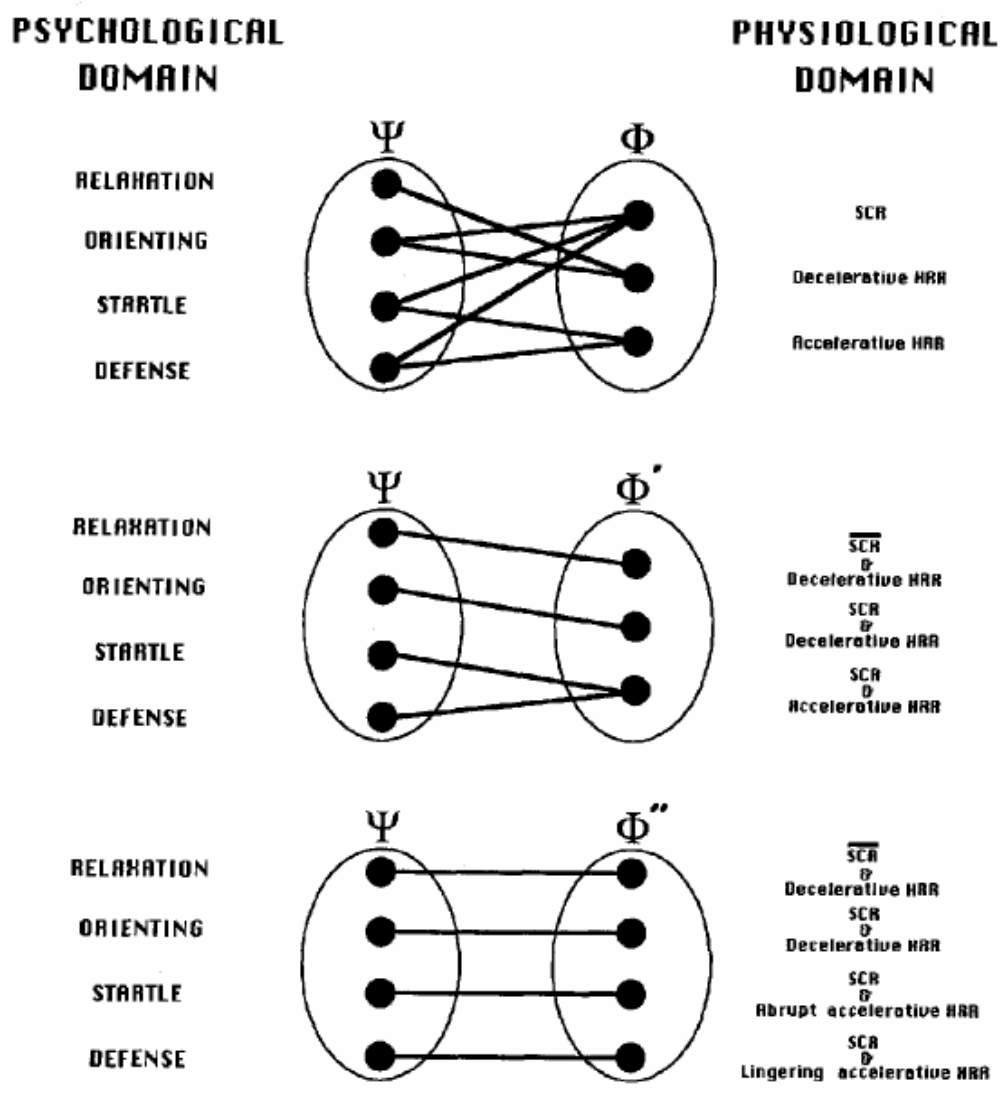

Figure 2-3: Relationship map of physiological events and psychological processes [23]

Figure 2-3, above, shows a depiction of relations between the psychological constructs of relaxation, orienting, startle, and defense and the physiological measures of heart rate (HR) and skin conductance response (SCR). Later, Cacioppo proposed that the use of mathematical combinations of physiological features could lead to a better discrimination of psychological states [23]. 
This dissertation assumed a mapping of psychological processes to the physiological domain and investigated affective assessment through features extracted from single signals individually, and through features mathematically combined from multiple physiological signals, using digital signal processing methods, and machine learning classification algorithms.

\subsection{Physiological Signals}

The autonomic nervous system, as a part of the peripheral nervous system, has far reaching effects, including its control of: pupil diameter, skin resistance, heart rate, digestion, respiration rate, breathing, etc.. Therefore, observation of these physiological variables is a critical mechanism to detect a user's affective state.

For the research work developed in this dissertation, the pupil diameter (PD) was chosen to be monitored in a non-invasive way for affective sensing. Two other physiological signals, galvanic skin response (GSR) and blood volume pulse (BVP) were also measured simultaneously for comparison purposes. Other physiological signals, such as the electrocardiogram (EKG) and the electroencephalogram (EEGs) were not chosen for this study due to the difficulty of measuring them within the "normal" human-computer interaction environment.

\subsubsection{Pupil Diameter}

The human pupil is a circular aperture at the center of the iris of the eye, through which light passes to the retina. Generally, the human pupil has a rapid reaction to stimuli (within 2 seconds). The pupil can constrict to $1.5 \mathrm{~mm}$, and dilate to as much as $9 \mathrm{~mm}$. 
These constriction and dilation are controlled by two opposing sets of muscles in the iris, the sphincter and dilator pupillae, which are governed by the sympathetic and parasympathetic divisions of the autonomic nervous system (ANS)[24]. The sympathetic ANS division, mediated by posterior hypothalamic nuclei, produces enlargement of the pupil by direct stimulation of the dilator muscles, which causes them to contract [25]. Pupil constriction is caused by excitation of the circular pupillary constriction muscles innervated by fibers from the parasympathetic division. The motor nucleus for these muscles is the Edinger-Westphal nucleus located in the midbrain. Sympathetic activation brings about pupillary dilation via two mechanisms [26]:

(i) An active component arising from activation of radial pupillary dilator muscles along sympathetic fibers;

(ii) A passive component involving inhibition of the Edinger-Westphal nucleus.

\subsubsection{Pupilllary Light Reflex}

For years, light intensity has been considered as the major determinant of the pupillary response. The pupil controls the amount of light coming into the eye by varying its own size (diameter). The light penetrating the retina travels through all the optic media in the eye, undergoing refraction and reflection at each medium boundary.

The pupil diameter or pupil size generally defines the optical properties of eye and also determines the amount of light falling on the retinal surface. The pupillary light reflex (PLR) designates the mechanism by which the human pupil changes its diameter inversely to the amount of light striking on the retina [26]. For example: under normal conditions, when the light intensity increases, an stronger activation of the 
parasympathetic division of the ANS will cause the constriction of the circularly arranged pupillary sphincter muscle (with a delay), which will result in a compensatory reduction of the pupil diameter [27]. Consequently, the reduced PD will cause a smaller pupil area, which will also reduce the light flux impinging upon the retina. The basic scheme of this behavior is shown as a negative feedback system in Figure 2-4.

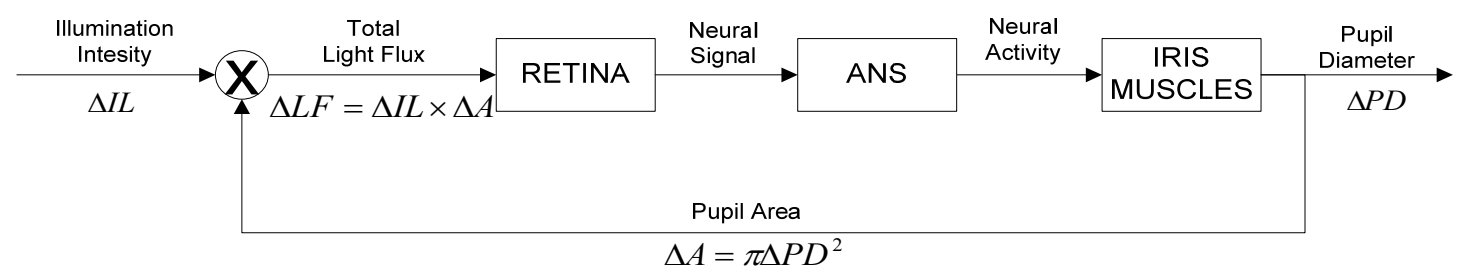

Figure 2-4: Functional representation of the PLR system.

In this way, the pupillary light reflex mainly functions to help the eyes adapt rapidly to variable lighting conditions. A typical PLR response for a step illumination increase can be shown in the following Figure 2-5.
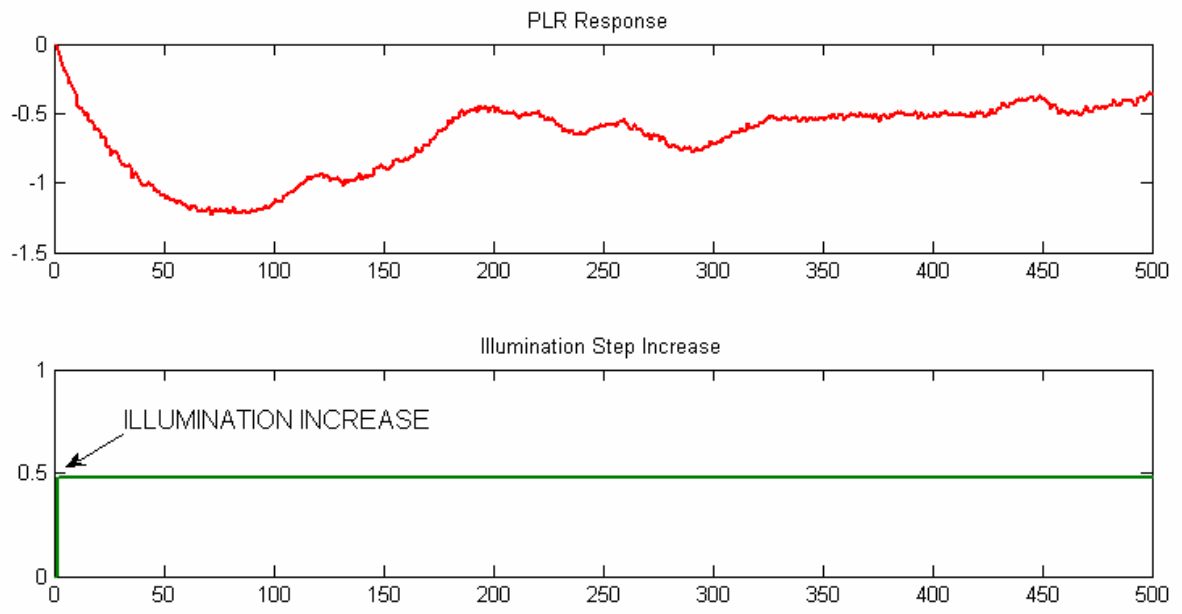

Figure 2-5: A typical PLR response 


\subsubsection{Pupillary Affective Response}

Several studies have pointed out that there are multiple factors that can cause pupil diameter increase. Anticipation of solving difficult problems, even thinking of performing muscular exertion, etc., will cause slight increases in pupil size.

As early as the 1850 s, Charles Darwin related pupil dilation to fear and other emotions in animals, in his book [28]. In 1978, Hess referred that other kinds of anticipation may also produce considerable pupil dilation [29]. Grings and Dawson also suggested that pupil size variation was also related to cognitive information processing. This, in turn, relates to emotional states (such as "stress") since the cognitive factors play an important role in emotions [30]. By the end of the 20th century, Kennedy and his colleagues have also proved that the pupil size increases as the mental workload does [31]. More recently, Partala and Surakka found, using auditory emotional stimulation, that the pupil size variation can be seen as an indication of affective processing [32].

The physiological foundation for the pupillary response to affective stimulation is that the pupil diameter is controlled by both the sympathetic and the parasympathetic divisions of the autonomic nervous system (ANS) [25]. Depending on a person's physical or mental state or the kind and intensity of stimulus applied to that person, the nervous system transmits neuronal signals that either cause the pupil to dilate or to contract [32]. Particularly, when the sympathetic division of the ANS is activated (e.g., due to stress), the pupil diameter is increased, and while the parasympathetic division predominates (e.g., during relaxation), the pupil size is reduced [33, 34]. As mentioned before, parasympathetic activation produces reduction of the pupil diameter by direct excitation 
of the circular pupillary constrictor muscle. In contrast, the pupil dilation promoted by the sympathetic division is contributed by central inhibition of the Edinger-Westphal complex of the oculomotor nucleus in the midbrain, which is the motor center for the parasympathetic pathway. Inhibition of this complex results in relaxation of the pupillary sphincter muscle, which leads to enlargement of the pupil [25]. In addition, sympathetic activation produces pupil dilation directly by contraction of the radial pupillary dilator muscle.

From the physiological description presented above, it is clear that pupil size variation can be also related to a subject's emotional state, since it impacts his/her ANS. This has led to the prospective monitoring of the pupil diameter variations to evaluate the affective state changes during human-computer interaction. In this study, a computer game was used to elicit "stress" in computer users, and the degree to which the pupil size can be associated with other physiological reactions relating to the appearance of this emotion were investigated.

\subsubsection{Pupil Diameter Measurement}

There are several techniques available for the pupil diameter measurement, which can be mainly classified into two types, intrusive and non-intrusive techniques. The intrusive techniques require attaching some device, such as contact lenses or electrodes, in physical contact with the subject. However, intrusive techniques would not be practical to implement during ordinary human-computer interactions. Therefore, this research focused on the use of non-intrusive pupil diameter measurement techniques. 
For over a century, the non-intrusive assessment of pupil diameter relied on photographic techniques, which are limited by their temporal resolution and lack of real-time capability. The eye of the subject would be first filmed during the experiment. Then, the film would be projected on a screen and the size of the pupil in each frame would be measured manually with a ruler or electronically with a photocell [30]. Beginning in the second half of the 20th century, electronic systems for imaging the pupil, including TV-based systems, were interfaced with newer computer technologies to provide more accurate assessment of pupil diameter. Currently, automatic vision-based instruments such as eye-tracking systems can be used to measure pupil size variations, which make use of illumination devices and cameras for image acquisition and processing.

In this study, the subject's eyes were monitored with a desk-mounted pupil diameter measurement eye tracking system, TOBII T60 (Figure 4-8) running on a PC to extract the values of pupil diameter with the sampling rate of $60 \mathrm{~Hz}$. The TOBII system estimates the diameter of both eyes every $1 / 60$ second, and the average (left + right / 2 ) was used as the instantaneous pupil diameter value, in each case.

\subsubsection{Galvanic Skin Response}

It is well known that when a person is experiencing stress or nervous tension, the palms of his/her hands tend to become moist. This is because of the activated sweat glands in these areas of the body, which have been filled with sweat (a hydrate solution of water and salt). Skin with higher water content will conduct an electric current more easily than dry skin, since a hydrated sweat gland provides less resistance for the passage 
of an electric current. In other words, increased activity in the sympathetic nervous system will result in increased hydration in the sweat ducts and on the surface of the skin, which will result in a decrease of skin resistance (increase in conductance). This emotional sweating can be measured by recording the conductance of a low-level electrical current as it is affected by changes in perspiration in a skin zone such as the hand or fingers [35] . This kindof change in electrodermal conductance has been termed as the galvanic skin response (GSR).

A single GSR response is shown in Figure 2-6.

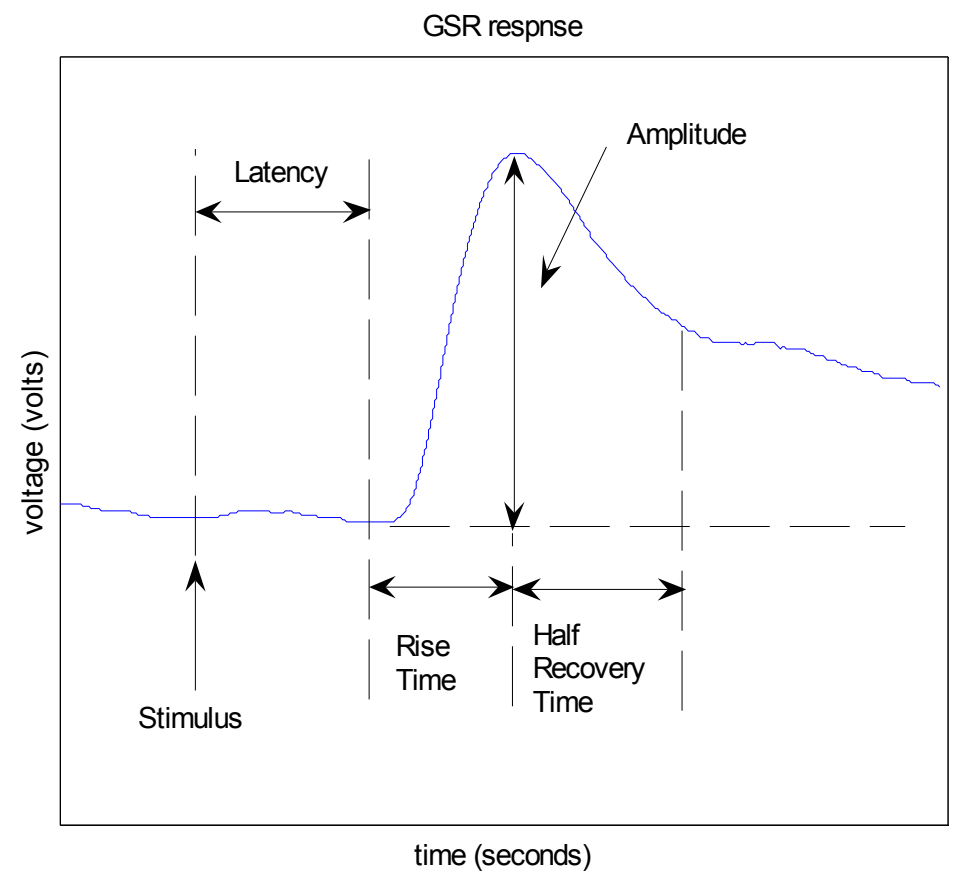

Figure 2-6: A typical GSR response

The GSR sensor selected for this research is a GSR2 module, by Thought Technology LTD (West Chazy, New York), which was placed at the middle and ring fingers of the subject. The GSR signal from this sensor was recorded at the sampling rate of 360 samples/second, with a data acquisition system. 


\subsubsection{Blood Volume Pulse}

As early as 1963, researchers found that the heart rate (HR) is a very selective autonomic indicator of psychological stress [36]. Normally, the HR can be measured by monitoring the blood volume pulse (BVP) or the electrocardiogram (ECG) of the subject.

Although, the ECG measurement is able to give a more precise estimate of instantaneous heart rate, by detecting sharp R-wave peaks, the application of ECG electrodes would be impractical for normal computer users. In contrast, the BVP measurement can be achieved merely by using a back-scatter photoplethysmograph (PPG) in the finger, to measure the blood volume in skin capillary beds. Therefore, in this study, BVP was selected to obtain the heart activity information with the advantage that it only requires the subject to attach a comfortable finger clip with a built-in BVP sensor.

A typical example of the BVP signal is shown in Figure 2-7

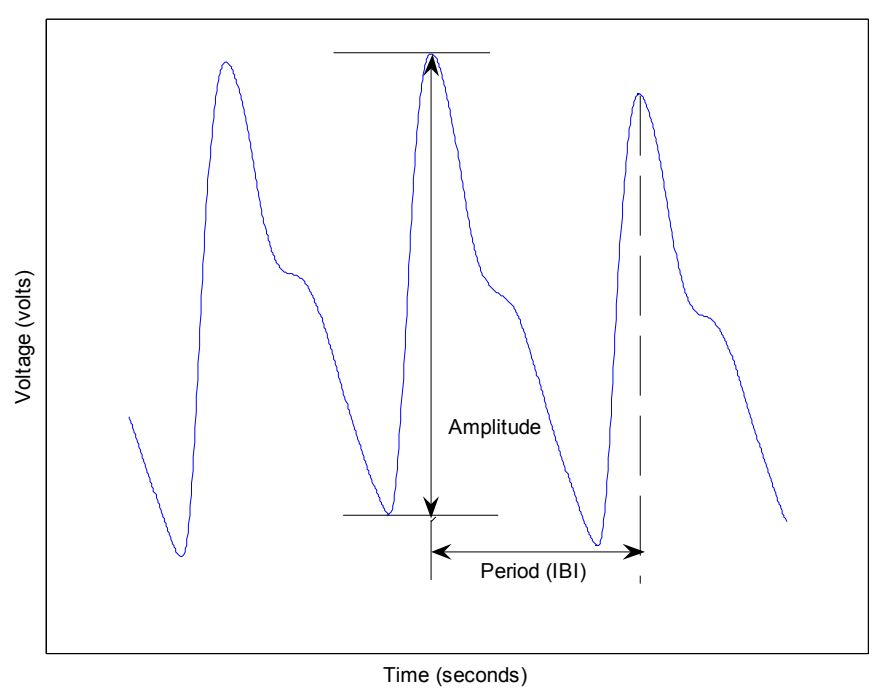

Figure 2-7: A typical BVP signal 
For the experiments performed in this research a Finger Clip Photoplethysmograph (UFI Model 1020-FC) was selected. This sensor was attached to the tip of the left index finger to measure the subject's BVP signal, which was sampled at the rate of 360 samples/second to ensure a high enough resolution.

\subsubsection{Additional Sensing Modalities}

Besides the PD, GSR and BVP signals, there are other sensing modalities that can be monitored for affective sensing, such as skin temperature (ST), blood pressure (BP), respiration, electromyogram (EMG) etc..

It has been proven that under sympathetic stimulation, the blood volume in the finger vessels is expected to decrease due to the vasoconstriction in the hairless areas of the hand but not in the hairy skin of the hand [37]. Accordingly, the finger temperature should exhibit a decrease as a response to stress, which implies that the finger temperature could be treated as an indicator of sympathetic activation.

Moreover, in 1980, Selye [38] mentioned in his book "Guide to Stress Research" that raised blood pressure was associated with the increase of stress. Therefore, the blood pressure could also be considered as another potential signal to use for affective sensing.

Similarly, faster and deeper respiration can be caused by both physical activity and emotional arousal, whereas slower and shallower respiration is expected to be observed during relaxation periods of study that include emotional changes in the subjects [39]. 
The electromyogram (EMG), detects surface voltages that occur when a muscle is contracted. It has been used in the studies of emotional valence and emotional stress by Lang, Cacioppo and Tassinary $[22,40]$.

In this dissertation, these signals were not selected for the purpose of affective sensing due to the fact that most of them require complex monitoring systems which are very impractical for application in the context of human computer interaction. The monitoring techniques used in this research are all non-invasive and fairly non-intrusive methods.

\subsection{The Concept of Stress}

Nowadays, "stress" has become a very popular word all over the world. However, its definition is still somewhat inconsistent across some disciplines (psychology, biomedicine or sociology). Although the term "stress" has appeared in the index of Psychological Abstracts since 1944 [41], the evolution in the study of psychology since then has not necessarily helped to fully clarify the meaning of this term.

Generally, modern stress research is based on the work of the American physiologist Walter B. Cannon and his colleagues, who considered stress as a syndrome of "fight-or-flight" activity in the sympathetic nervous system [42]. In 1970, McGrath suggested that stress was "a (perceived) substantial imbalance between demand and response capability, under conditions where failure to meet demand has important (perceived) consequences" [43]. The debates surrounding the characterization of stress have never stopped over the years. For example, Cox [44] stated that "Stress can be 
defined as a psychological state which is part of and reflects a wider process of interaction between individuals and their work environment".

There have been numerous experimental paradigms used for the investigation of the stress phenomenon. The following is a brief review of key relevant studies.

One approach is the scanning test [45], which presents participants with pictures that have sexual, aggressive or neutral content and records the subject's eye movement. It is proposed that anxious people are likely to be hyper-vigilant for threatening stimuli, which means that they will scan the environment for threatening information more rapidly and more thoroughly than non-anxious people. On the other hand, the distractibility task was also used to investigate the distracting influence of threatening and neutral stimuli by Mathews and MacLeod [46] in a dichotic listening experiment, which requires participants to "shadow" (speak aloud) spoken passages that are played through one channel of a pair of stereo headphones. They found that stressed people were more easily distracted by threatening stimuli than by non-threatening stimuli, and that this occurs through pre-conscious processes.

Many of these stress studies appear to suggest that stressed or anxious people perform cognitive tasks differently from non-stressed people. For the experimentation involved in this dissertation a Stroop Color-Word Interference Test was chosen. This paradigm allows for the elicitation of both "relaxation" and "stress" during the experiment, which will be described in detail in chapter 4. Ultimately, the goal of this research is the differentiation of these two states from each other through the processing of the monitored pupil diameter signal. 


\subsection{Previous Experiment Designs for Affective Sensing}

Previous sections have outlined why a psychophysiological signal (e.g. pupil size, skin conductance, heart rate, respiration, blood volume pressure) is expected to reflect involuntary ANS reactions. To the extent that distinct emotions prepare the organism for distinct behavior, they ought to result in distinct physiological features. This is the fundamental rationale for using specific indicators derived from physiological signals to recognize a particular affective state.

Recently, a variety of different research approaches have been developed for affective sensing under experimental conditions, and there is now an abundant literature on this topic. The following is a brief review, focusing on stress studies.

A implicit human-robot cooperation system was developed by Rani and her colleagues in 2002, which is based on psychophysiological measures [47]. The participants in their experiment played video games with varying difficulty levels of mathematical problems and performed an anagram task with varying difficulty to stimulate mental stress.

Heart rate, blood volume pulse, and skin conductance signals were monitored by Ward and Marsden [48] to assess user's responses within web page interaction cases. The specific experimental context focused on interactions with "poorly designed" software.

Researchers from Prendinger's group implemented a mathematical quiz game where subjects were instructed to sum up five successively displayed numbers and were then asked to subtract the $i$-th number of the sequence ( $i \leq 4)$. Subjects competed for 
the best score in terms of correct answers and time while their GSR and BVP signals were monitored for the purpose of emotion recognition.

Partala and Surakka [32] presented an empirical study of the affective sensing, via a specific psychophysiological measure, pupil size, using the international affective digitized sounds (IADS) system to elicit different affective status including stress. Their study demonstrated that the pupil size in human subjects increases following emotionally significant auditory stimuli. These findings, coupled with the increasing effectiveness of eye-tracking systems, show promise for the development of real-time user affect assessment methods.

\subsection{Summary}

In this chapter, the physiological background related to the signals monitored in the project (PD, GSR and BVP) has been described, followed by the introduction of the affective computing concepts and a basic description of the autonomic nervous system. In addition, some modalities used in other research studies were presented as well. The literature related to the concept of stress was briefly reviewed. Finally, some experiments related to emotion recognition (especially those related to stress detection) were summarized. 


\section{CHAPTER 3}

\section{METHODOLOGY}

The aim of this research is the development of a system for computer user stress detection through pupil diameter monitoring, to fulfill the affective sensing requirements of a prospective affective computing system. It is known that both illumination intensity and affective state changes can modify the size of the pupil. However, for this study, only the pupil diameter variations associated with the computer user's affective state are of interest. Therefore, we propose to use an AIC system with the HITV adaptive algorithm to remove the PLR component from the measured PD signal of the subject, by involving an independent measurement of the light variations experienced by the user. Accordingly, the output of the AIC, the MPD signal, will be an estimation of the PAR. To implement the actual detection of stress, additional post-processing is applied to the MPD signal obtained from the AIC, resulting in the PMPD signal. Ultimately, a feature derived from the PMPD signal is normalized and applied to a classification algorithm for stress recognition. On the other hand, classification of the same states of the computer user based on features extracted from the simultaneously monitored GSR and BVP signals was also investigated, to compare the performance of these two approaches to the affective assessment.

\subsection{Framework of Research}

The framework for the research is summarized in Figure 3-1 below, which provides a schematic overview of the human-machine interaction context where affect recognition is attempted. 
The complete affective sensing system is described in the big box on the left region in this figure. If the affect sensing process is able to determine the emotional state of the computer user, the next affective controlling action would be decided based on the affective state information along with other environmental inputs. The activity between the human being and the machine is shown as an interaction loop.

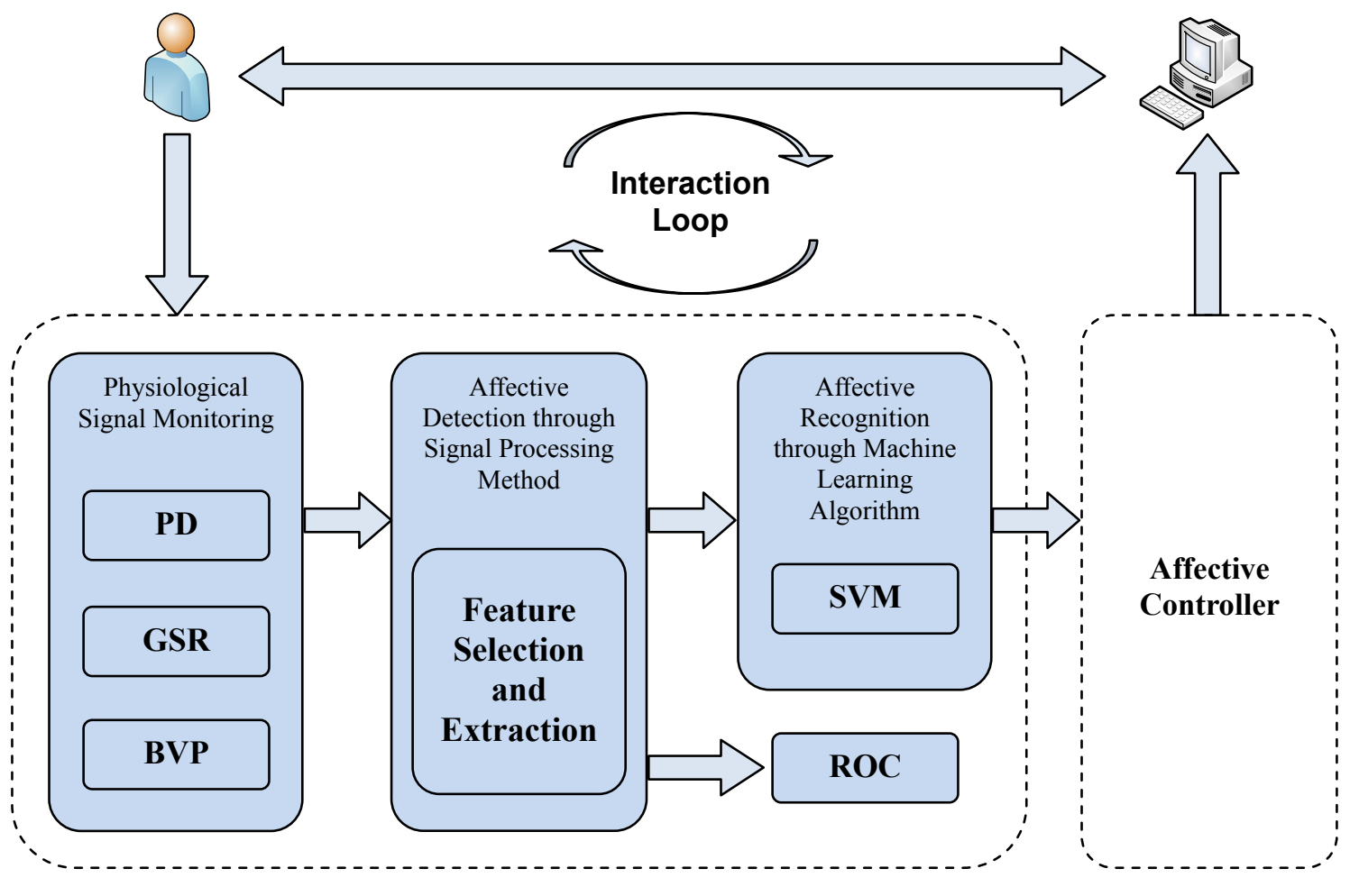

Figure 3-1: Human-computer interaction framework. This doctoral research focuses on the section of this diagram enclosed by the biggest box, to the left of the diagram.

As indicated in this diagram, the scope of the work developed in this dissertation is composed of three modules:

(i) Physiological signal monitoring;

(ii) Affective detection through signal processing methods;

(iii) Affective recognition through machine learning algorithm. 
In the first module, the physical changes of the computer user are recorded by sensing the physiological signals chosen: PD, GSR and BVP. Although affective sensing through the PD signal is the main concern of this study, the other two signals were recorded simultaneously to provide a basis for comparison between the novel approach (PD) and approaches previously proposed by other researchers (GSR and BVP). After signal collection, these 3 signals were analyzed with different digital signal processing techniques, in the second module, to extract important emotion-related features. The physiological features obtained are the input for the third module, which is the actual affective recognition system. An SVM pattern recognition algorithm was evaluated for the implementation of this module, to identify and classify the probable affective state associated with the physiological signals recorded. And a ROC analysis of these feature indicators was performed, as well.

The focus of this dissertation is on affective sensing rather than on affective controlling, (block shown in bottom-right of Figure 3-1), where the development of an "affective controller" would modify the way in which the machine participates in the interaction, based on the results of the affective sensing block.

Additionally, it should be pointed out that the emphasis of this project was on answering the following questions, in a general sense:

Q1 Is it feasible to process the pupil diameter signal to minimize the impact of illumination intensity on pupil diameter changes? 
Q2 Can an Adaptive Interference Canceller (fed with the measured PD and the illumination intensity signal) yield a modified pupil diameter signal that is less impacted by the pupillary light reflex?

Q3 Will the PD signal provide a better assessment of the affective status of the subject than other physiological signals such as GSR and BVP?

To obtain an initial answer to these questions, which could provide the directions for future developments and implementations, the experiments in this dissertation were performed under artificially controlled conditions, constraining the influence of environmental changes or additional effects on the subjects to just those planned as part of the experimental protocol. It is clear that the conditions for everyday human-computer interaction are not normally controlled in these ways.

Finally, it should also be noted that in this attempt to provide initial answers to the questions posed above, no specific emphasis has been placed on the possibility of implementing the analysis methods developed for on-line, real-time performance. All the processing mechanisms described in the following chapters were implemented off-line, on data recorded during experimental sessions that took place before the processing.

\subsection{Adaptive Interference Canceller System}

An AIC system is an adaptive mechanism known to be able to remove an unwanted component from a signal, under the assumption that a sample of the unwanted component can be obtained. The kernel of this mechanism is an adaptive filter, which processes the adaptive algorithm to implement the function of noise canceling. 


\subsubsection{Adaptive Interference Canceller}

The basic AIC is a two-input-one-output system, whose structure is illustrated in Figure 3-2. An uncorrelated noise signal transmitted over the top channel is the primary input, $d(k)$, and the bottom channel receiving the signal, which is uncorrelated with the desired signal $s(k)$, but correlated with the interference $n(k)$, is the reference input $r(k)$. The output of AIC, $e(k)$, is the channel for the expected noise-removed signal.

As described in the previous paragraph, the mathematic schemes of the AIC can be written as:

Primary Input:

$$
\begin{gathered}
d(k)=s(k)+z(k) \\
r(k)=n(k)+u(k) \\
e(k)=d(k)-y(k)=d(k)-\hat{z}(k)=\hat{s}(k)
\end{gathered}
$$

Reference Input:

System Output:

where,

$\checkmark s(k)$ is the signal of interest;

$\checkmark z(k)$ is the interference in the primary sensor;

$\checkmark n(k)$ is the actual source of the interference;

$\checkmark u(k)$ is the measurement noise.

The practical objective of this AIC is to produce a system output $e(k)$, which is a best estimation of the interested signal $s(k)$. This objective is accomplished by feeding the system output (error) back to the adaptive filter and using it to adjust the filter through an adaptive algorithm to minimize the error signal. 


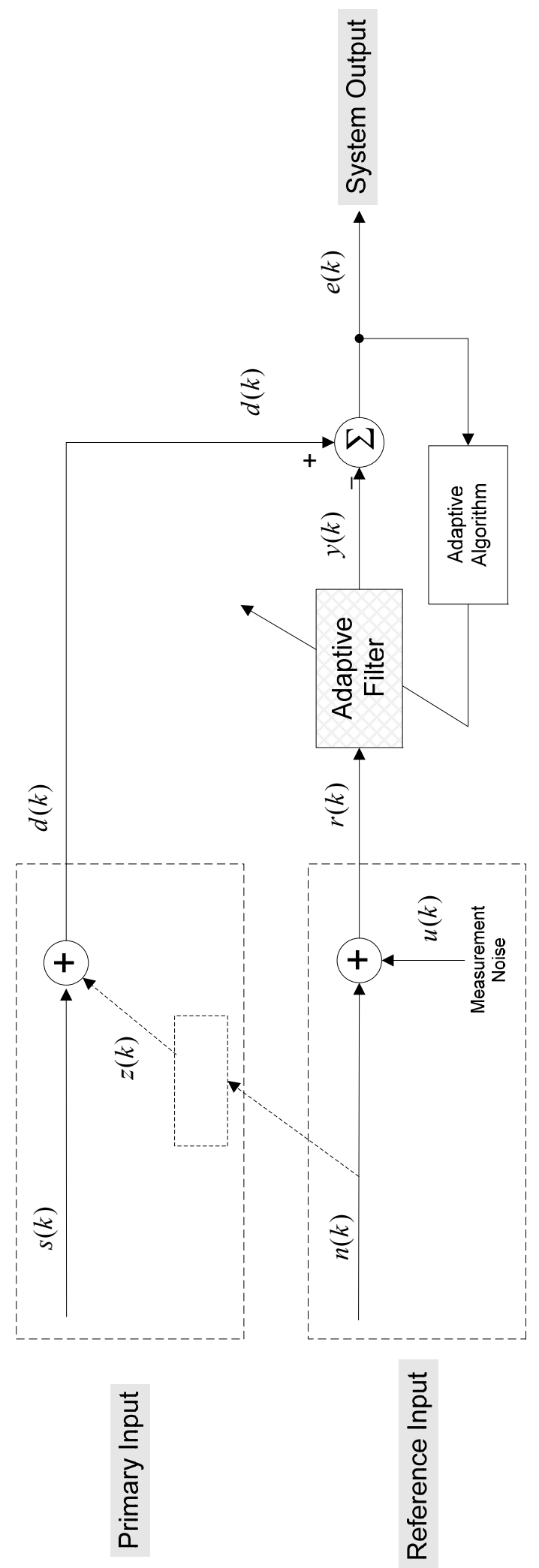

Figure 3-2: AIC system structure 


\subsubsection{Adaptive Filter}

In the AIC system, the essential part is an adaptive transversal filter (ATF), which is similar to a finite impulse response (FIR) filter. However, its coefficients are adjustable weights, $w(k)$ that are changed with every new sample acquired and processed (shown in Figure 3-3 below).

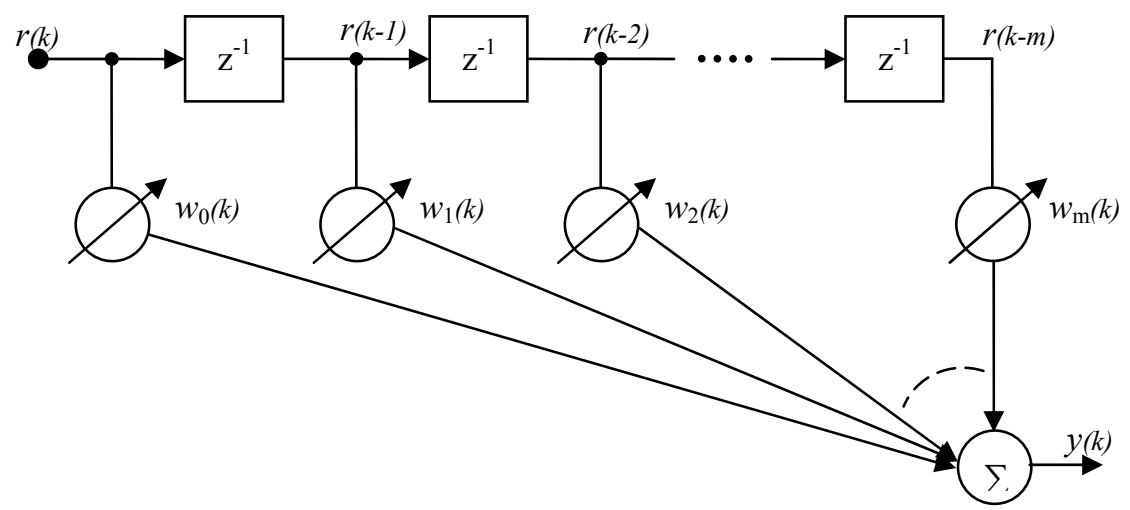

Figure 3-3: Adaptive filter mechanism [49]

The function of the ATF is processing the reference signal $r(k)$ to produce an output $y(k)=\hat{z}(k)$ that is an approximation of $z(k)$. The state space model of the ATF is given by $[50]$ :

$$
\begin{gathered}
w(k+1)=w(k)+\Delta w(k) \\
d(k)=r(k)^{T} w(k)+v(k) \\
z(k)=r(k)^{T} w(k)+v(k) \\
v(k)=s(k)+v(k)
\end{gathered}
$$

In the equations,

\footnotetext{
$\checkmark w(k)=$ system state vector, is the ATF coefficient vector of size $m \times 1 \quad(m=$ order of ATF);

$\checkmark d(k)=$ measurement sequence, is the observed PD signal;
} 
$\checkmark \quad z(k)=$ sequence to be estimated;

$\checkmark \quad \Delta w(k)=$ process noise vector, represents the time variation of the ATF weights $w(k)$;

$\checkmark \quad v(k)=$ measurement noise vector, includes $s(k)$ (PD- driven by affective changes) and model uncertainties $v(k)$;

$\checkmark \quad r(k)=\left[r_{k}, r_{(k-1)}, \ldots \ldots, r_{(k-m+1)}\right]^{T}$ is the interference vector of size $m \times 1$.

\subsubsection{Adaptive Algorithms}

In the adaptive filtering problem, usually, we assume an FIR model. However, the true system may involve feedback and hence modeling errors of unknown statistical properties may be introduced into the estimation problem. Therefore, it is essential to select an adaptive algorithm that possesses good robustness properties.

In this study, the $\mathrm{H}^{\infty}$ time-varying (HITV) and LMS adaptive algorithms were compared to perform the adaptation of the AIC by modeling the PLR system (Figure 3-4). In the AIC system, if the signal $s(k)$ is eliminated (i.e., no affective contribution to PD changes is considered), the primary input of AIC would merely be the interference component $z(k)$. Then, the output of AIC should converge to zero theoretically and the final weights of the adapted filter would indicate the impulse response of the PLR system mediating between $n(k)$ and $z(k)$. 


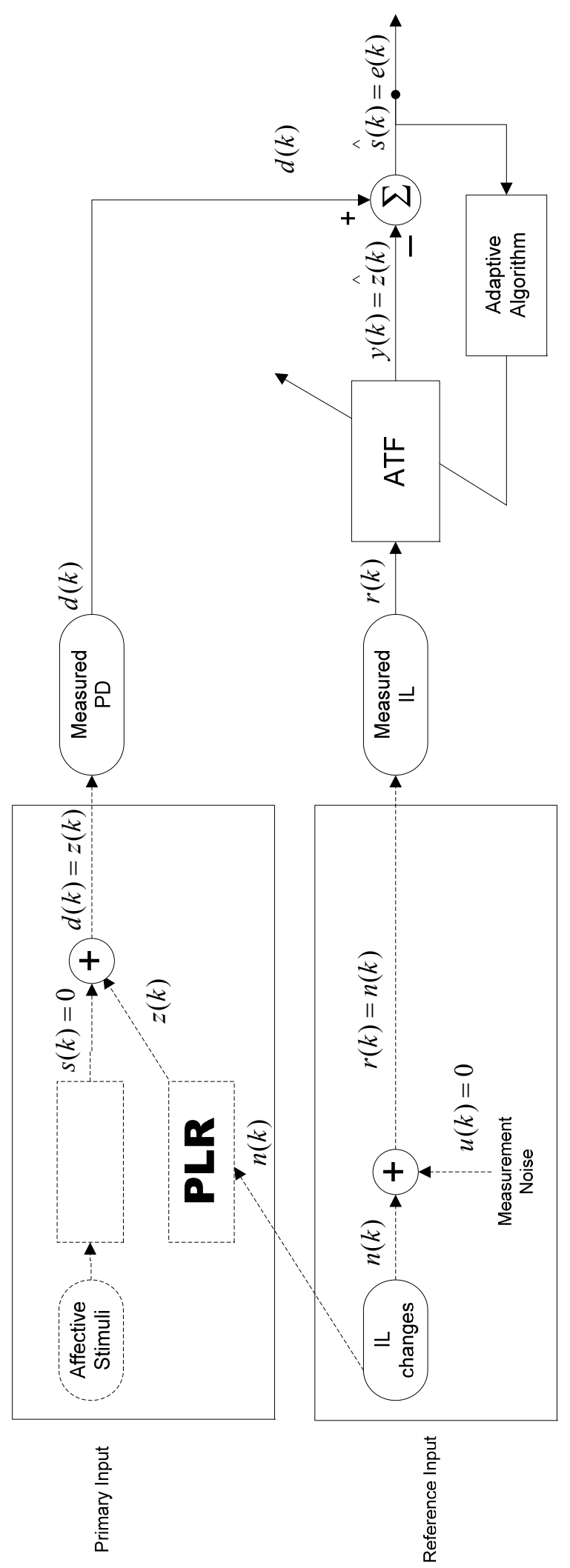

Figure 3-4: Adaptive algorithms compare by PLR system modeling with AIC 


\subsubsection{1 $H^{\infty}$ Time-Varying Algorithm}

The $\mathrm{H}^{\infty}$ approach introduced in robust control theory is based on the hypothesis

that the resulting minimax estimation technique would be less sensitive to model uncertainties and parameter variations than conventional techniques [50]. This method safeguards against the worst-case disturbances and therefore makes no assumption on the (statistical) nature of the signals. In addition, the robustness of the HITV algorithm is encouraged by minimizing the maximum energy gain from the disturbances to the estimation errors with the following solutions [51]:

$$
\begin{gathered}
\tilde{P}^{-1}(k)=\mathrm{P}^{-1}(k)-\varepsilon_{g}^{-2} \mathrm{r}(k) \mathrm{r}^{T}(k) \\
g a(k)=\frac{\tilde{\mathrm{P}}(k) \mathrm{r}(k)}{1+\mathrm{r}^{T}(k) \tilde{\mathrm{P}}(k) \mathrm{r}(\mathrm{k})} \\
\hat{w}(k+1)=\hat{w}(k)+g a(k)\left(y(k)-\mathrm{r}^{T}(k) \hat{w}(k)\right) \\
P(k+1)=\left[P^{-1}(k)+\left(1-\varepsilon_{g}^{-2}\right) r(k) r^{T}(k)\right]^{-1}+\gamma_{0} \\
P(0)=\eta I=\Pi_{0} \quad \Upsilon_{0}=\rho I
\end{gathered}
$$

Here, $g a(k)$ is the gain factor; $\varepsilon_{g}, \eta$ and $\rho$ are positive constants. Note that $\rho$ reflects a priori knowledge of how rapidly the state vector $w(k)$ varies with time, and $\eta$ reflects the a priori knowledge of how reliable the initial estimate available for the state vector $w(0)$ is. 


\subsubsection{LMS Algorithm}

The LMS Algorithm, the most popular adaptive algorithm, has been widely used in adaptive signal processing because of its simplicity and ease of computation. It adjusts its coefficients to minimize the mean-square error between its output and that of an unknown system, which is given by

$$
\hat{w}(k)=\hat{w}(k-1)+\beta r(k)\left(y(k)-r^{T}(k) \hat{w}(k-1)\right)
$$

where $\beta$ is the convergence rate parameter.

\subsubsection{Comparison of HITV and LMS Algorithms}

Based on the state-space model of the ATF in the AIC, the HITV and LMS adaptive algorithm formulations were implemented in MATLAB, for the purpose of minimizing the estimation error with the appropriate settings of filter order and parameters. The result of HITV adaptive algorithm showed better performance in the modeling of the negative feedback PLR system, which encouraged its utilization in this dissertation for PLR interference removal and PAR isolation.

Prior to its application to the AIC, the measured PD signal was first analyzed by an algorithm that detected the interruptions due to eye blinks (identified by a non-zero value in the validity code provided by the Tobii system) and compensated them by linear interpolation of the lost values. Figure 3-5 shows the results of the blink-removal process (bottom pane), on a raw PD signal (top pane), using the combined validity codes from both eyes (middle pane). 

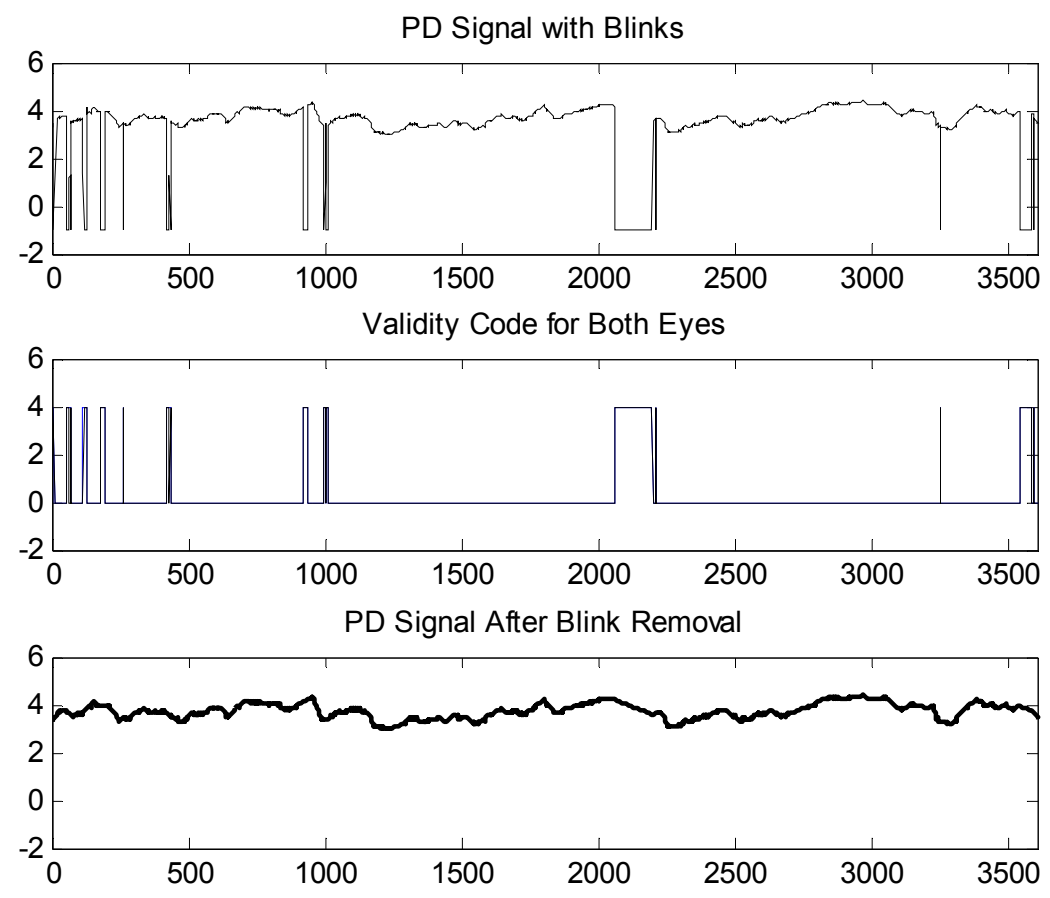

Figure 3-5: Adaptive algorithm comparison - blink removal

The PD signal obtained after blink-removal was processed by the AIC system as the primary input signal $d(k)$ and the reference input signal $r(k)$ was the measured illumination intensity signal, as shown in Figure 3-6. Then, the prospective output of this adaptive filter system is an error signal that is expected to converge to zero and the weights of the adapted filter are expected to indicate the impulse response of the system model.

The AIC outputs resulting from the HITV and the LMS algorithm adaptations are shown in the bottom pane of Figure 3-6. The adaptation parameters used were $\eta=0.002$, $\rho=0.0001, \varepsilon_{g}=2.0$ for HITV and $\beta=0.002$ for LMS. 

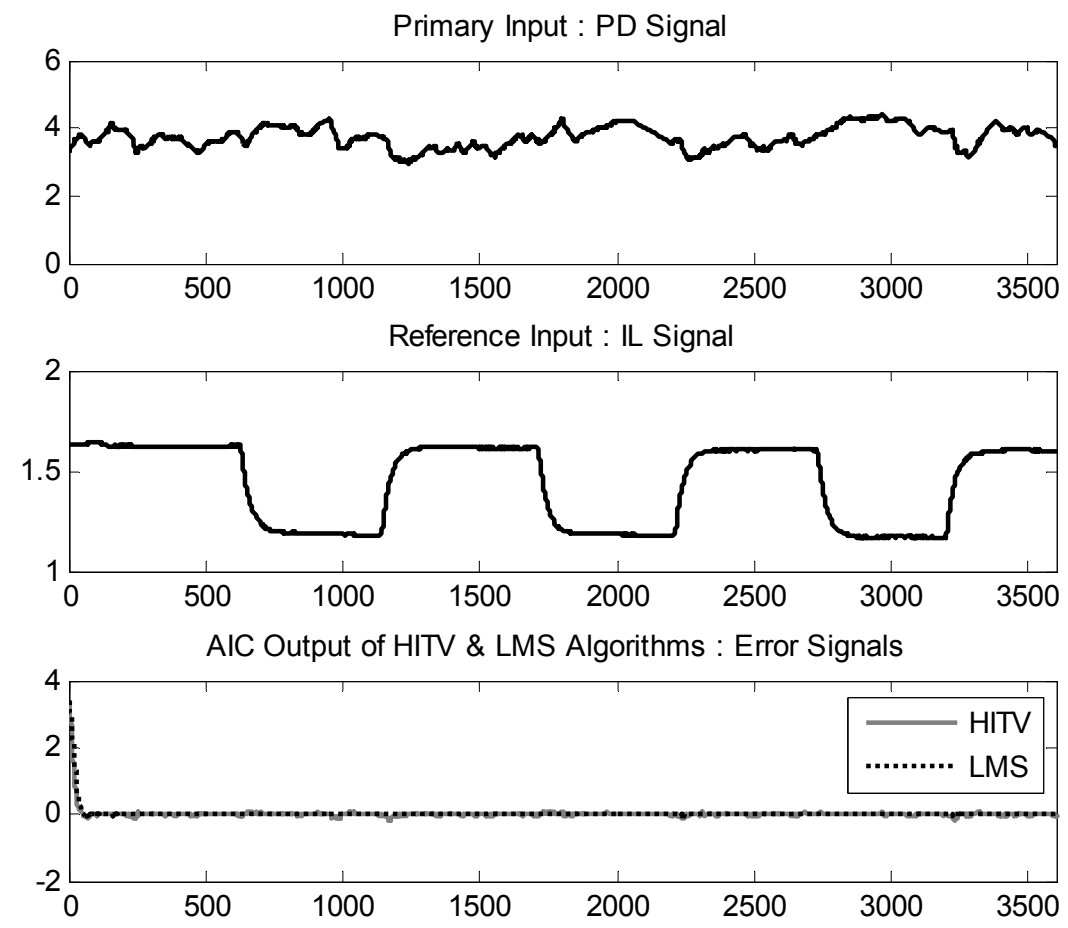

Figure 3-6: Adaptive algorithm comparison - AIC implementation with HITV \& LMS algorithms

The time required for convergence to an error level of less than 0.05 and the mean square error (MSE) of both algorithms are compared in Table 3-1.

Table 3-1: Compare of HITV \& LMS adaptive algorithms - AIC results

\begin{tabular}{|c|c|c|}
\hline & HITV & LMS \\
\hline \multirow{2}{*}{ Time for convergence to 0.05} & 47 samples & 50 samples \\
\cline { 2 - 3 } & 0.783 second & 0.833 second \\
\hline MSE & 7.84 e-4 & 1.73 e-5 \\
\hline
\end{tabular}

The value of the MSE was calculated by the equation below ( $L$ is the length of the input signal).

$$
M S E=\frac{\sum_{k=1}^{L}(y(k)-d(k))^{2}}{L}=\frac{\sum_{k=1}^{L} e(k)^{2}}{L}
$$


The adapted weights after the implementation of HITV and LMS adaptive algorithms are compared in Figure 3-7.

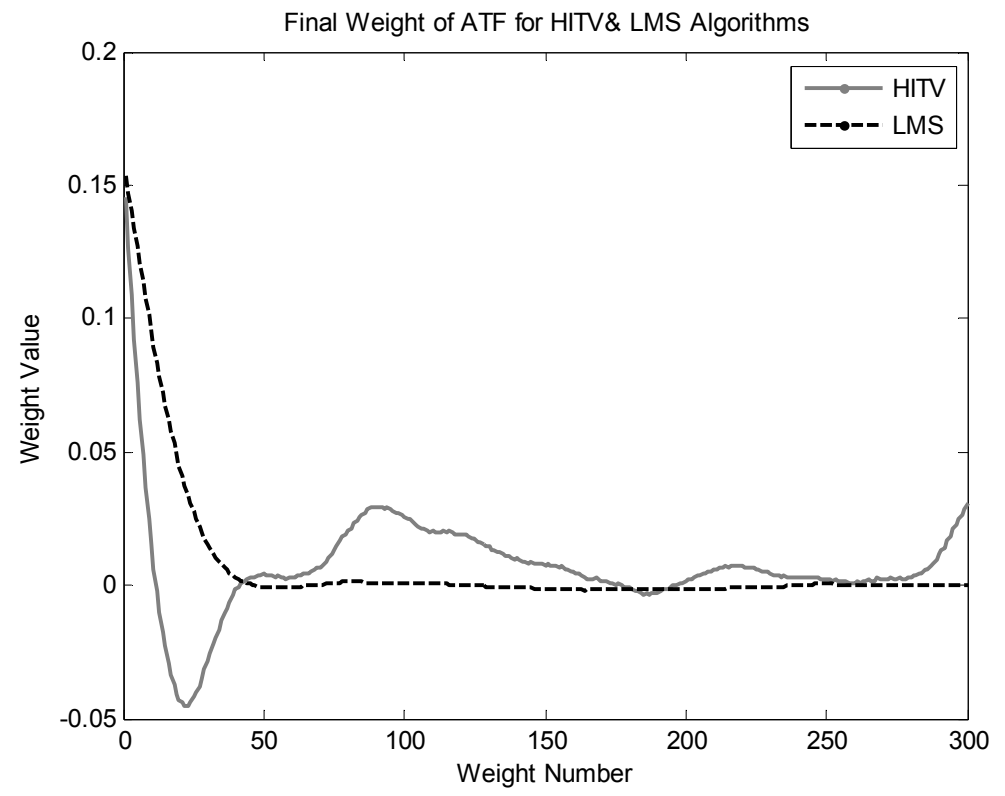

Figure 3-7: Adaptive algorithm comparison - final weights after HITV and LMS adaptation

The results in Table 3-1 and Figure 3-7 show that, although, the LMS algorithm yields a smaller mean square error, the HITV adaptive filter converges slightly faster and results in a small MSE of $7.84 \mathrm{e}-4$, which is also very close to zero .

In separate research efforts, the PLR system has been studied previously by some other researchers. For example, using control system analysis, Lawrence Stark [52] proposed a linearized model (shown in Figure 3-8) with a third-order transfer function for the PLR, applicable to relatively small changes of illumination. 


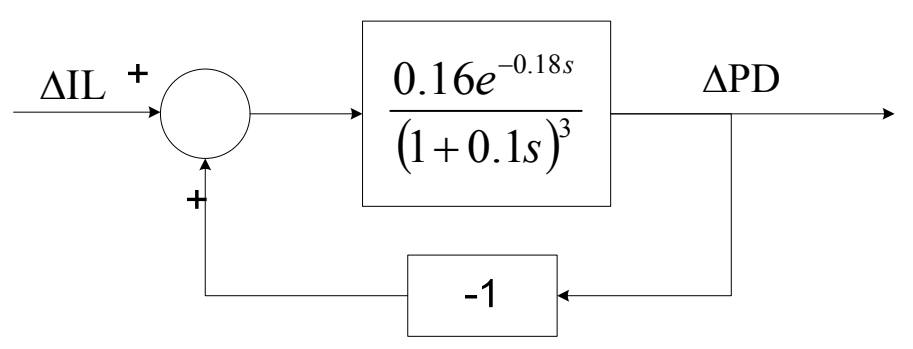

Figure 3-8: PLR system model by Stark

In a separate research effort, Clarke, Zhang and Gamlin [53] investigated the static and dynamic characteristics of the PLR system in an alert rhesus monkey (shown in Figure 3-9). With the illumination stimuli (bottom trace), the pupil response (middle trace) can be consider as the step response of the PLR system, so that the velocity (top trace) represents the impulse response of the PLR system.

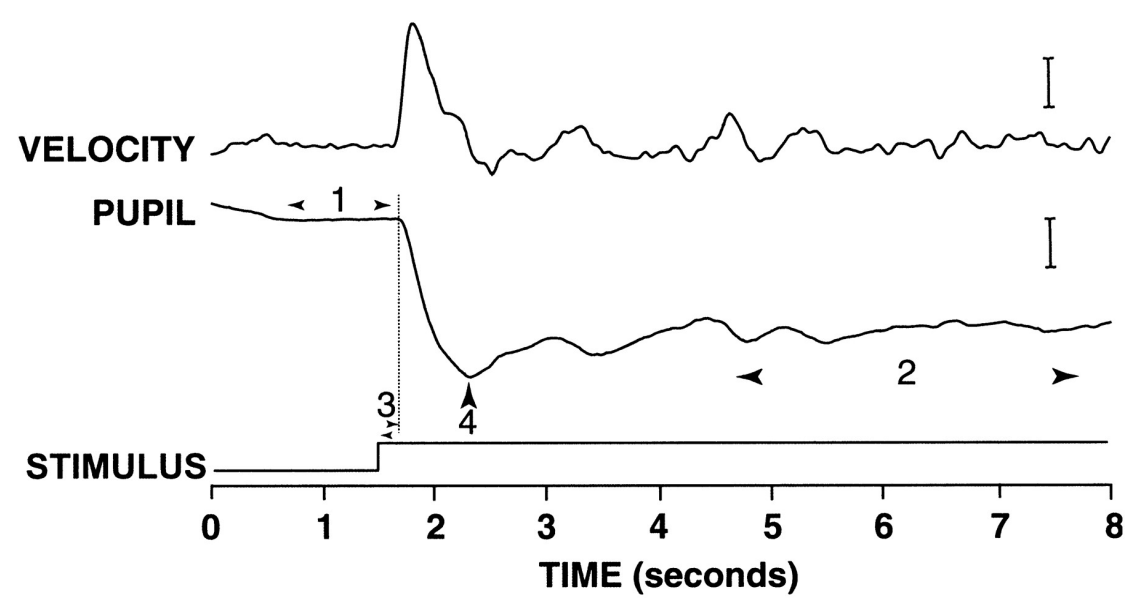

Figure 3-9: PLR system model by Clarke, Zhang and Gamlin [53]

Comparing Stark and Clarke's approach with the system modeling attempt in this study, the HITV method seems to be able to capture more of the complex dynamics of the PLR system, while the LMS approximation converges to a simpler representation of the system, which is, perhaps, not fully modeling the dynamics of the PLR phenomenon. The result of the adaptive algorithm comparison suggested an advantage in using the 
HITV adaptive algorithm, which encourages the use of this algorithm for PD processing to separate the PAR from PLR for the affective sensing.

\subsection{PD Signal Processing and Feature Extraction Method}

As shown in Figure 3-10, the PD signal is mainly processed by an AIC system

adapted by the HITV adaptive algorithm. Additionally, initial pre-processing stages were applied to the raw PD signal to remove the blink and artifact interference. The last step, post-processing of MPD, was developed to achieve better indication of the PAR.

\subsubsection{AIC Application with HITV for PD}

In this study, we define the primary input of the Adaptive Interference Canceller as the "Measured PD" signal, which is composed by the signal of interest $s(k)$ (PDdriven by PAR) and the interference $z(k)$ (PD- driven by PLR). In general, the reference input may contain the actual source of the inference $n(k)$ (illumination changes) and measurement noise $u(k)$. In this model, with the assumption that the measurement noise is negligible, the independent measurement of illumination in the neighborhood of the eye of the subject ("Measured IL") is used as the reference input. The core component of the AIC system, i.e., the ATF, emulates the transformation of the illumination to pupil diameter changes, which would convert the noise $n(k)$ into a close-enough replication of the PLR-driven components of PD (the output $y(k))$. Therefore, the system output, $e(k)$ (MPD), would be the estimation of the desired signal $s(k)$, representing exclusively the affective responses of the computer user. 


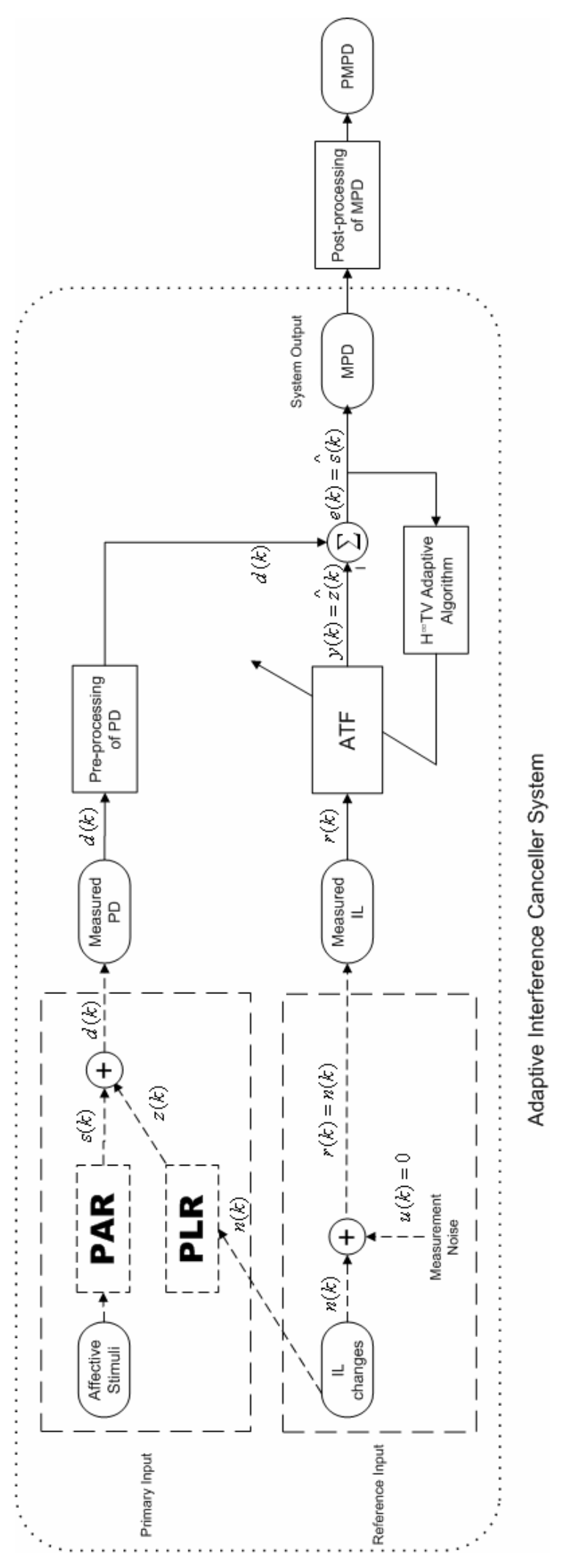

Figure 3-10: PD processing diagram 
The "Measured IL" signal was first processed by a median filter with length of 50 . Then, max-min normalization was applied through equation (3.13) to constrain the illumination signal to a range of $[0,1]$. When the normalized illumination value surpassed a threshold value 0.6, it was considered a "high IL" value and, accordingly, the $\rho$ parameter of the HITV algorithm was set to a higher value.

$$
I L_{\text {norm }}=\frac{I L-I L_{\text {median }}}{I L_{\text {max }}-I L_{\text {median }}}
$$

An example of this time varying relationship is shown in the Figure 3-11, below.
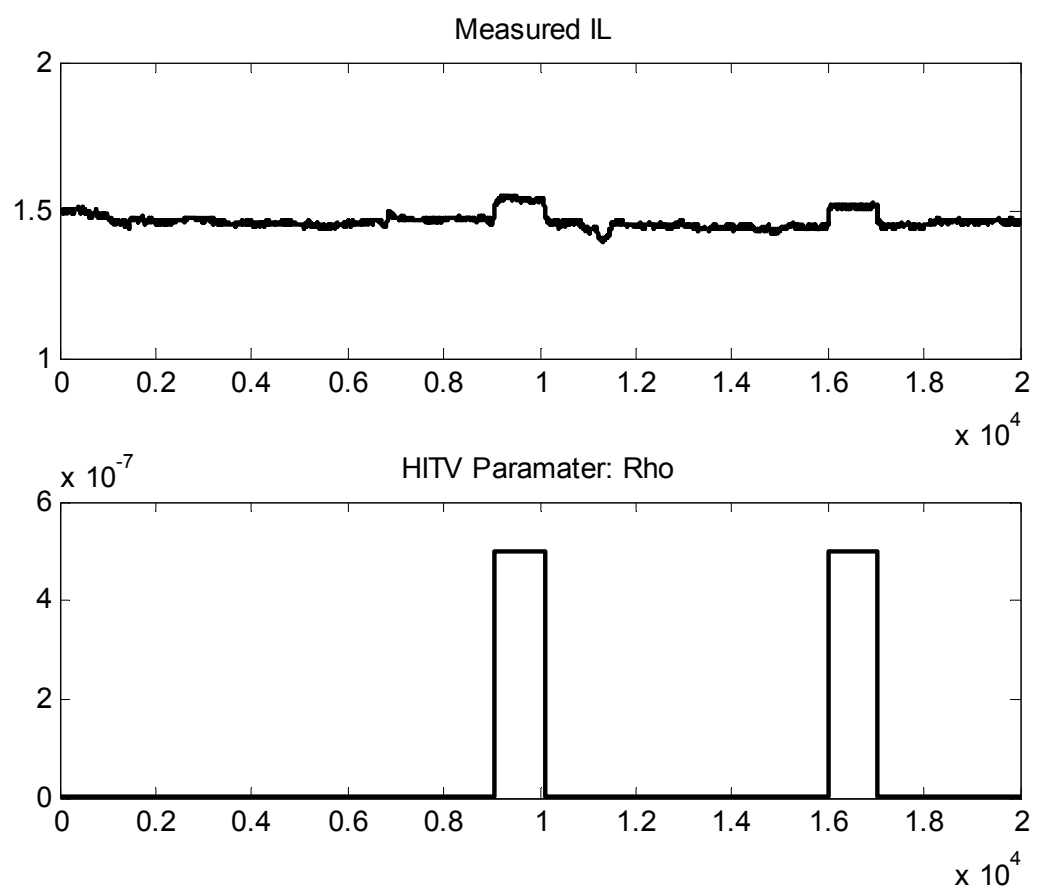

Figure 3-11: HITV parameter RHO changed along with "Measured IL"

\subsubsection{Pre-processing of PD}

Prior to its adaptive interference canceller application, the "Measured PD" is transformed by a pre-processing algorithm. 
The first step is detecting the interruptions due to eye blinks and artifacts, which were identified by values between " 1 " and "4" (not " 0 ") in the validity code provided by the pupil measurement system (TOBII T60 Eye Tracker), and then compensating them by linear interpolation of the lost values.

After that, a low-pass finite impulse response (FIR) digital filter was designed to filter out the blink responses, i.e. the oscillations in pupil diameter observed after every blink of the subject due to the PLR response caused by the temporary decrease in the light intensity on the retina that occurs during the blink. This filter is designed with 512 delay units and a cutoff frequency of $0.13 \mathrm{~Hz}$ which is meant to suppress the pupil diameter blinking response waveforms, while preserving the PD level shifts associated with affective changes in the subject. The effect of this filter is relevant only for the sporadic time intervals that immediately follow every blink (a typical blink rate for humans is reported as 17 blinks per minute [54]). The reason to choose the FIR filter instead of an IIR (infinite impulse response filter) is that the phase response of the FIR filter is linear within the passband of the filter, so that the waveform of the filtered signal will not be distorted.

The MATLAB command for the design of this filter is

$$
\mathrm{B}=\operatorname{fir} 1\left(\mathrm{~N}, \mathrm{~W}_{\mathrm{n}}\right)
$$

where $N$ is the filter length and $W_{n}$ is the normalized cutoff frequency with 1.0 corresponding to half the sample rate. In this study, $\mathrm{N}=512$ and $W_{n}=\frac{0.13}{60 / 2}=0.0043$ with the sampling frequency of 60 samples/second. The frequency response of this filter 
is shown in Figure 3-12. It is obviously that the phase response in the passband of this filter is linear so that the signals in this band will keep their original shape without any phase distortion. Since the length of this FIR filter was 512 (i.e., there are 512 delay units inside this filter), the filtered signal was also shifted forward by 256 sampling intervals to maintain the correct time alignment with the rest of the signals in the AIC system.
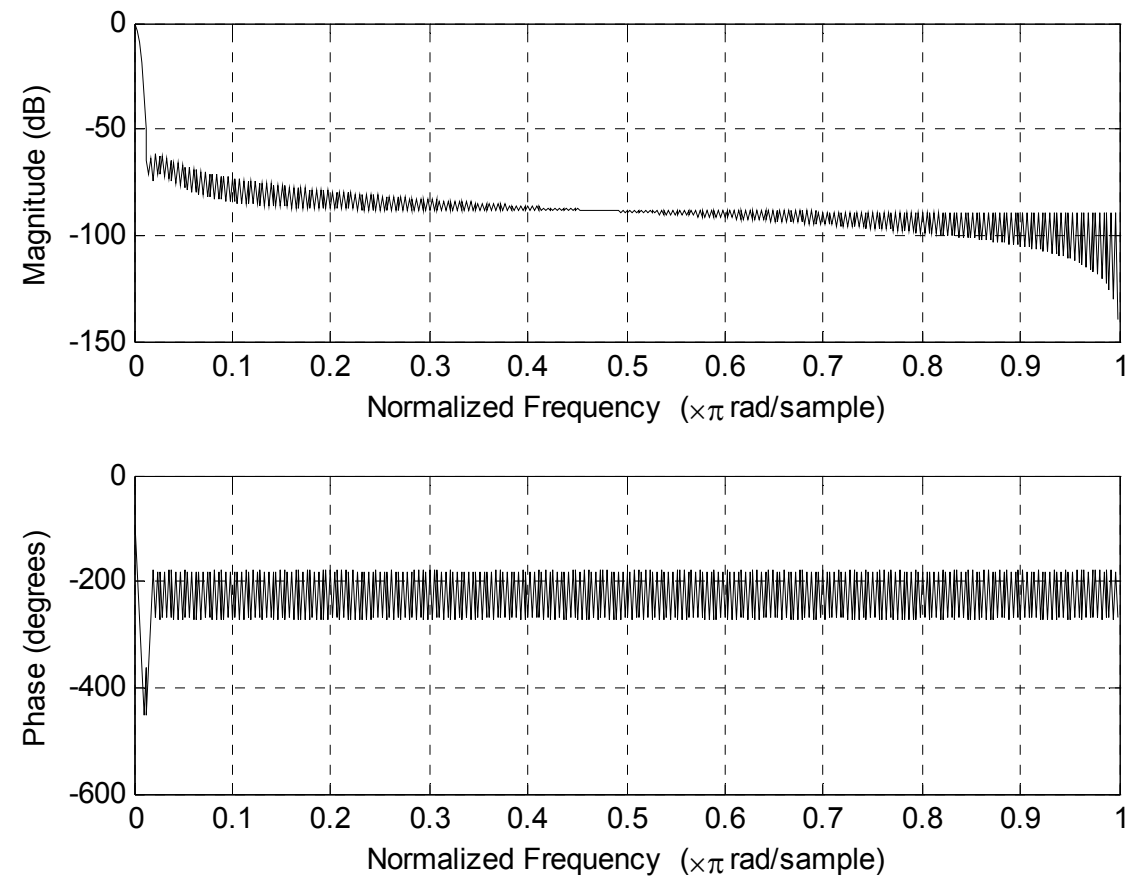

Figure 3-12: Frequency response of the FIR filter used for PD pre-processing

\subsubsection{Post-processing of MPD}

As reported by Beatty and Lucero-Wagoner [24] and others, the increase in sympathetic activation associated with the affective state of "stress" is expected to cause a dilation of the pupil .Therefore, the negative portions of the MPD signal, at the output of AIC, which represent temporary decreases in pupil diameter, were zeroed-out to isolate significant MPD increases, which indicate the emergence of stress in the subject. Then, a sliding window with a width of 900 samples is further applied throughout the 
non-negative MPD signal to calculate the median value within each window. The resulting signal is termed the processed MPD, or "PMPD" signal.

\subsubsection{Features Extraction from the PMPD Signal}

In general, there are three measures that are typically extracted from pupil diameter measurements: mean pupil dilation, peak dilation, and latency to peak [55]:

$\checkmark$ Mean pupil dilation - average of the relevant number of data points in the measurement interval;

$\checkmark$ Peak dilation - the maximal dilation obtained in the measurement interval of interest;

$\checkmark$ Latency to peak - the amount of time elapsed between start of the measurement interval and emergence of the peak dilation.

For this research, the stimuli are not considered as discrete stimuli, and, therefore, the peak of pupil dilation and the latency to peak were not considered to be applicable features to describe the changes of pupil size dilation from one affective state to the other. In consequence, only the mean value of the PMPD was calculated for the affective sensing purpose.

\subsection{GSR Signal Processing and Feature Extraction Method}

The first step to process the raw GSR signal is to find the GSR phasic responses (discrete responses that appear as transient increases in the GSR signal). A typical example of a measured GSR response is shown in Figure 2-6. A low-pass FIR filter with 64 delay units and a cutoff frequency of $2 \mathrm{~Hz}$ was designed to filter out the recording 
noise while retaining the GSR response waveforms, whose bandwidth is approximately 1 Hz. Since the length of the FIR filter was 64 , the filtered signal was shifted forward by 32 sampling intervals to maintain the time information. After filtering, the mean value of this signal was obtained as one of the GSR features.

Then, the signal was normalized using $\mathrm{z}$-score normalization to make the signal have zero-mean and unit variance with equation (3.14).

$$
z n=\frac{i-\mu}{\sigma}
$$

where $i$ is the signal, $\mu$ is the mean of the signal and $\sigma$ is the standard deviation of the signal.

To isolate the phasic responses from the background, Ktonas' 7-point Lagrangian interpolation algorithm [56] was applied immediately after. For the explanation of this algorithm, let $g[n]$ represent the discrete time signal obtained by sampling the GSR signal.

(i) The first derivative is computed using the 3-point Lagrangian interpolation approach:

$$
g^{\prime}[n]=\frac{g[n+1]-g[n-1]}{2 h}
$$

where $h$ is the sampling interval.

(ii) The second derivative is calculated with the 5-point second-order fit differentiation:

$$
g^{\prime \prime}[n]=\frac{2 g^{\prime}[n+2]+g^{\prime}[n+1]-g^{\prime}[n-1]-2 g^{\prime}[n-2]}{10 h}
$$


(iii) Substituting equation (3.15) into equation (3.16), yields 7-point operator:

$$
g^{\prime \prime}[n]=\frac{2 g[n+3]+g[n+2]-2 g[n+1]-2 g[n]-2 g[n-1]+g[n-2]+2 g[n-3]}{20 h^{2}}
$$

Then, $g "[n]$ was multiplied by a constant value of 200 to re-scale this result. By thresholding the resulting signal, the responses in the GSR signal could be localized and counted. The number of the responses in a measurement segment could was used as the second GSR feature. Additionally, the amplitude and the rising time from each GSR response were also recorded for GSR feature extraction. Furthermore, the total area under the rising time curve was recorded to represent the energy of each GSR response.

\subsection{BVP Signal Processing and Feature Extraction Method}

The original BVP signals were filtered using an FIR filter which had similar configuration as for GSR filtering except that the cutoff frequency is $6 \mathrm{~Hz}$. To keep the same waveform and preserve time alignment, the output of this 64 order low-pass filter was shifted forward by 32 samples as well.

As described in chapter 2, the heart rate variability (HRV) information can be obtained from the high resolution BVP signal recorded. A typical example of a measured BVP signal is shown in Figure 2-7. The HRV studies are based on the inter-beat interval (IBI) data analysis. The filtered BVP signal was differentiated using the forward difference method.

$$
f d(n)=b(n+1)-b(n)
$$

By searching the local maximum and minimum around the segment of highest first derivative, the peak and valley of each BVP beat can be obtained. The heart rate was 
calculated as the inverse of the peak to peak interval and the amplitude was the difference between the peak and the valley. The average amplitude of the BVP beats in the measurement interval under analysis was treated as one of the physiological features extracted from the BVP signal.

The number of samples between two peaks of the signal was calculated and divided by the recording sampling frequency of 360 samples/second, the peak to peak interval can be represented in milliseconds, and the progression of the values calculated in this way defines the inter-beat interval (IBI) sequence. Then, the IBI signal was treated as a time series to perform power spectrum density (PSD) analysis in order to extract the HRV information, which is typically analyzed in three frequency bands: very low frequency (VLF) $(0.00-0.04 \mathrm{~Hz})$, low frequency (LF) $(0.05-0.15 \mathrm{~Hz})$ and high frequency (HF) $(0.16-0.40 \mathrm{~Hz})$. In this study, PSD analysis was performed on the IBI signals from each experimental segment, using the Welch method. The ratio defined by the IBI power in the low frequency range to the IBI power in the high frequency range was recorded as the second BVP feature, for the corresponding segment.

Additionally, the mean value and standard deviation of the IBI sequence within each segment of analysis were also extracted as additional features to represent the cardiac characteristics of each BVP interval.

\subsection{Features from PD, GSR, and BVP}

In this dissertation, the specific aim is to evaluate the affective assessment performance of a single-feature stress detector, derived from PMPD. The efficiency of this single-feature detector is also compared with 9 other single-feature detectors derived 
from measured GSR and BVP signals. These 10 (in total) feature detectors were assessed in terms of the individual discriminating power to differentiate between the "relaxed" affective states and the "stressed" states of the computer user.

Table 3-2 shows the features obtained from each of the signals recorded in the experiments. The processes to obtain the 10 features in this table have been outlined in the previous sections, and are reflected in their names.

Table 3-2: Features extracted from the bio-signals

\begin{tabular}{|c|c|c|}
\hline Biosignal & Features & Definition \\
\hline $\begin{array}{c}\text { PD } \\
\text { (1 feature) }\end{array}$ & PMPDmean & Average value of the non-negative PMPD signal \\
\hline \multirow{5}{*}{$\begin{array}{c}\text { GSR } \\
\text { (5 features) }\end{array}$} & GSRmean. & Average value of the GSR samples \\
\hline & GSRnum & Number of the GSR responses \\
\hline & GSRmeanAmp & Mean value of the amplitude of each GSR response \\
\hline & GSRrisingTime & Rising time of each GSR response \\
\hline & GSReng & Energy of GSR (total under the rising time curve) \\
\hline \multirow{4}{*}{$\begin{array}{c}\text { BVP } \\
\text { (4 features) }\end{array}$} & BVPmeanAmp & Mean value of the amplitude of each BVP beat \\
\hline & BVPl2h & $\begin{array}{l}\text { Low frequency to high frequency rate for Power spectral } \\
\text { density of inter-beat interval(IBI) (the time in } \\
\text { milliseconds between two normal, consecutive peaks in } \\
\text { the BVP) sequence }\end{array}$ \\
\hline & BVPIBImean & Mean value of the IBI \\
\hline & BVPIBIstd & Standard deviation of IBI sequence \\
\hline
\end{tabular}




\subsection{Data Normalization}

Before attempting to use the 10 single-feature detectors, individually, to identify "stressed" and "relaxed" in the experiment, the 9 features extracted from the BVP and GSR signals were normalized according to the following (i), (ii) and (iii) steps. The "PMPDmean" feature was only normalized through steps (ii) and (iii), equations (3.19) \& (3.20), since the illumination levels used during the preliminary introductory period, prior to the actual experiment, was variable and different from the illumination levels used during the actual experiment. As such, this preliminary period cannot be used as a suitable baseline for pupil dilation.

(i) Reference the features to their baseline level;

$$
Y_{s}=\frac{X_{s}}{X_{r}}
$$

Here $X_{s}$ is the raw feature value during "relaxation" and "stress" segments, and $X_{r}$ is the corresponding feature value during the preliminary period, prior to the test part in the experiment. (This was done only for GSR and BVP features)

(ii) Minimize the impact of individual subject responses dividing each feature by the sum of all segment values;

$$
Y_{s}{ }^{\prime}=\frac{Y_{s i}}{\sum_{i=1}^{6} Y_{s i}}
$$

(iii) Normalize all data to the range of $[0,1]$ with max-min normalization.

$$
Y_{n o r m}=\frac{Y_{s}^{\prime}-Y_{s}^{\prime}{ }_{\text {min }}}{Y_{s}^{\prime}{ }_{\text {max }}-Y_{s}^{\prime}{ }_{\text {min }}^{\prime}}
$$




\subsection{Stress Detection}

This study proposes to detect the emergence of "stress" states in a computer user through the feature "PMPDmean" derived from the PD signal using a machine learning algorithm, i.e., a support vector machine (SVM), as a classifier, to differentiate between "stress" and "relaxation" states. Subsequently, multi-feature classifiers, also implemented through support vector machines (SVMs), were used to process the features from the GSR and BVP signals, with and without the PMPD feature. Additionally, the individual "stress"/"relaxation" discriminating potential of each one of these 10 features (1 from PD, 5 from GSR and 4 from BVP) were studied through their receiver operating characteristic (ROC) curves.

\subsubsection{Classification Fundamentals}

A classification model is a mapping of instances into a certain class/group. The classifier result will frequently be a real value (continuous output) in which the classifier boundary between classes must be determined by a threshold value.

This dissertation, addresses a two-class classification problem ("relaxation" and "stress"), in which the outcomes are labeled either as positive class (P) for "stress" or negative $(\mathrm{N})$ class for "relaxation". There are four possible outcomes from the two-class classifier. If the outcome from the classifier is $\mathrm{P}$ and the actual value is also $\mathrm{P}$, then it is called a true positive (TP); however if the actual value is $\mathrm{N}$ then it is said to be a false positive (FP). Conversely, a true negative (TN) appears when both the classification outcome and the actual value are $\mathrm{N}$, and false negative (FN) indicates a classification 
outcome $\mathrm{N}$ while the actual value is $\mathrm{P}$. The four outcomes can be formulated in a $2 \times 2$ contingency table as follows:

Table 3-3: Two-class classification scheme

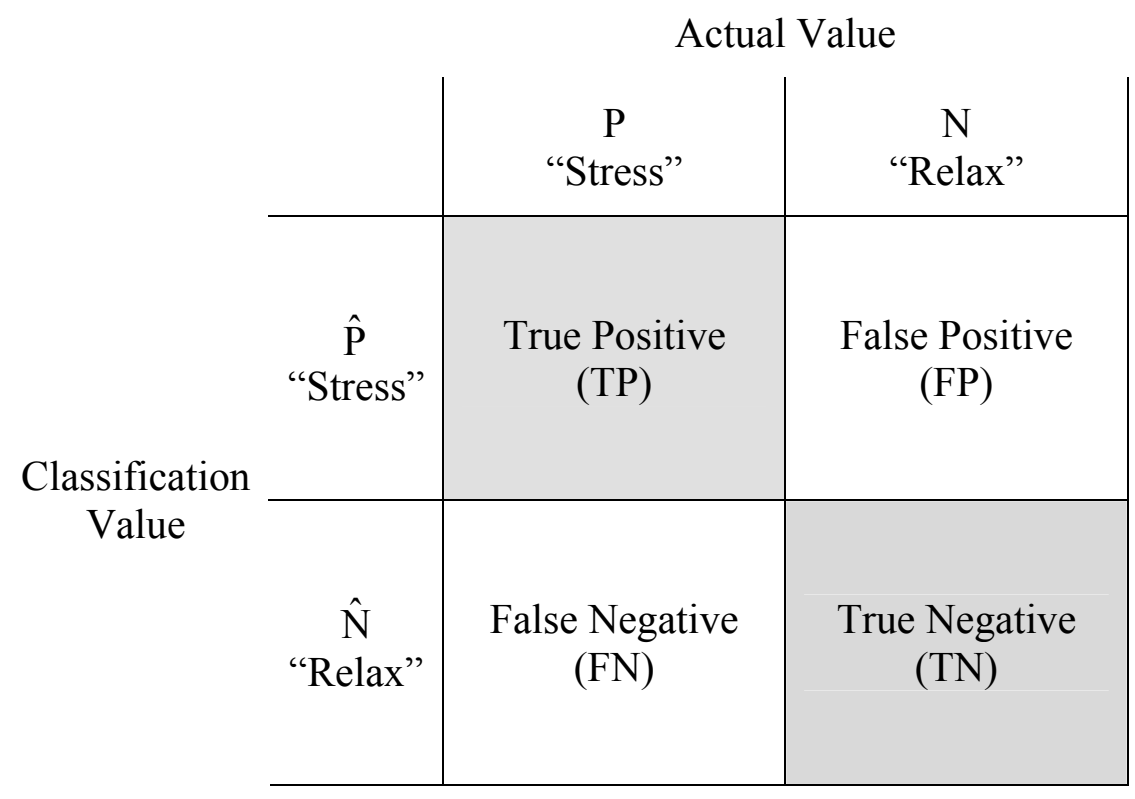

A successful classifier is characterized by generating a high percentage of TP and TN (the shaded part in Table 3-3), but as little FP \& FN as possible.

\subsubsection{Support Vector Machine (SVM)}

Support vector machines (SVMs) are a set of supervised machine learning methods used for classification and regression. Viewing input data as two sets of vectors in an n-dimensional space, an SVM will construct a separating hyperplane in that space, one which optimally separates the data into two categories. The performance of SVMs have been verified in a variety of research areas including pattern recognition [57], text categorization [58], face recognition [59], computer vision [60] and others [61, 62]. 
Classifying data is a common need in machine learning. Suppose some given data points each belong to one of two classes, and the goal is to decide which class a new data point will be in. In the case of SVMs, with a set of given examples, $X=\left\{x_{i}: x_{i} \in R^{n}\right\}$, and the corresponding known labels (targets), $Y=\left\{y_{i}: y_{i} \in\{\right.$ possible types $\left.\}\right\}$, a discriminant function, $f: R^{n} \rightarrow$ \{possible types\}, has to be learned ( $n$ is the dimension of the input vector). At the same time, the number of misclassifications of $f$ on the training set $\{X, Y\}$ is minimized by the learning machine (algorithm) during this training phase. The practical appeal of these methods is in their capacity to define the class of previously unseen samples (test set).

The aim of SVM is to construct a (linear) discriminant function for the data points in feature space in such a way that the feature vectors of the training samples are separated into classes, while simultaneously maximizing the distance of the discriminant function from the nearest training set feature vector. Therefore, the most important part of SVM classification is to find a suitable discriminant function (kernel function) that can not only capture the essential properties of the data distribution, but also prevent the over-fitting problem. Furthermore, non-linear discriminant functions can also be applied in the SVM classifiers by mapping the input vectors into a different feature space using a mapping function $\Phi: x_{i} \rightarrow \Phi\left(x_{i}\right)$, and using the vectors, $\Phi\left(x_{i}\right), x_{i} \in X$, as the feature vectors, $\Phi\left(y_{k}\right), y_{k} \in Y$, as the target vector.

The kernel function used by the SVM algorithm can be written as:

$$
K\left(x_{i}, y_{k}\right)=<\Phi\left(x_{i}\right) . \Phi\left(y_{k}\right)>
$$


The most popular kernels used in SVMs are:

Linear:

$$
K(X, Y)=X \bullet Y
$$

Polynomial:

$$
K(X, Y)=(X \bullet Y+1)^{p}
$$

Radial basis function $(\mathrm{RBF})$ :

$$
K(X, Y)=\exp \left(-\gamma\|X-Y\|^{2}\right), \quad \gamma>0
$$

Gaussian:

$$
K(X, Y)=\exp \left(\frac{-\|X-Y\|^{2}}{2 \gamma^{2}}\right)
$$

Here, $\quad \gamma$ and $p$ are kernel parameters. The "•" used in these equations represents the dot product of two vectors.

To obtain a more realistic assessment of the performance of an SVM, a $k$-fold cross validation [63] method is commonly used, which separates given data sets into two parts: the training sets and the test set, where the label of test sets is considered unknown in the classifier training. Then, the accuracy on these sets can more precisely reflect the performance that can be expected in classifying unknown data. This cross-validation procedure can prevent the over-fitting problem, allows the SVM to train and test on different samples and obviates the need to test on unknown samples whose labels (targets) may be uncertain.

In this dissertation, the stress classification based on the features derived from the PD, GSR and BVP signals during individual experimental segments was studied using SVM classifiers. Although, numerous implementations of SVMs are currently available, including svmTorch [64], SVMLight [65], Gist [66], and LibSVM [67], the "SPIDER" software package for MATLAB was used, which can be freely downloaded from http://www.kyb.mpg.de/bs/people/spider/. The kernel functions chosen for use with the 
SVM classifier were Linear, Polynomial, RBF and Gaussian kernels. According to the cross validation strategy, the original data is first divided into 20 equal subsets. Sequentially, one subset is tested using the classifier trained on the remaining 19 subsets. This process is repeated until every instance has been used exactly once for testing. The overall success rate for a classifier was evaluated as the number of correct classifications divided by the total number of feature sets tested:

$$
\text { AccuracyRate }=\frac{T P+T N}{T P+F P+T N+F N}
$$

\subsubsection{Receiver Operating Characteristic (ROC)}

In signal detection theory, a receiver operating characteristic (ROC) curve, is a graphical representation that illustrates the trade-off made in a classifier between two operating characteristics, true positive rate (TPR) and false positive rate (FPR), as its discrimination threshold is varied. The TPR refers to the fraction of all positive cases correctly classified; correspondingly, the FPR reflects the false alarm level, i.e., the fraction of negative cases incorrectly classified as positive.

In the ROC plot, the origin of the coordinate axes represents the case when the classifier produces no false alarms but detects no positive cases, at the lowest sensitivity level (i.e., setting the threshold at the highest possible value of the detection signal). In contrast, the top right-hand corner represents the situation when the classifier reports all positive cases correctly (true positive rate $=1$ ), but all negative cases incorrectly (because

it generates a false alarm on each negative case, i.e., false positive rate $=1$ ), when the threshold is set at the lowest possible value of the detection signal. 
Generally, the ROC curves "start" at the coordinate origin, $(0,0)$ and "end" at the upper-right corner $(1,1)$. The trajectory between these points followed by a given ROC, and consequently, the "Area under the ROC" (AUROC) are indicators of the discriminating power of the detection signal being thresholded. A classification system based on a highly discriminating detection signal (in this study, a single feature) will have one or several threshold levels that map close to the upper-left corner of the ROC, at ( 0 , 1). This location represents a high sensitivity (large true positive rate) and also a high specificity (low false positive rate). If that is the case, the AUROC will come close to encompassing the full 1.0-by-1.0 square. That is, the AUROC will approach the ideal value of 1 . On the other hand, a ROC curve that remains close to the bottom-right corner implies a lack of discriminating power in the detection signal (feature), and will result in a smaller value of the corresponding AUROC.

The ROC curve for each of the 10 features was developed in this study with the "stress" state set having an ideal classifier output of 1, and the "relaxation" state set having an ideal classifier output of -1 . Each point of the ROC curves is determined by comparing the value of the single detection signal to a specific threshold and determining which portion of the "positive classifier outputs" match the ideal output (1) and which portion of the "negative classifier outputs" are in disagreement to the ideal output (-1). These "portions", expressed as fractional numbers, yield the coordinates of the ROC point (false positive rate, true positive rate) for the threshold value tested. The process was carried out using the ROC MATLAB ${ }^{\circledR}$ scripts provided by Dr. Gavin C. Cawley (University of East Anglia, Norwich, UK) in his web site http://theoval.sys.uea.ac.uk/MATLAB/default.html. These scripts not only sweep the 
complete range of normalized threshold values, [0, 1], and draw the ROC, but additionally estimate a "convex hull" that fits the actual ROC points calculated.

\subsection{Overall Research Method Design}

Figure 3-13 provides an overview of the research methodology employed for this dissertation.

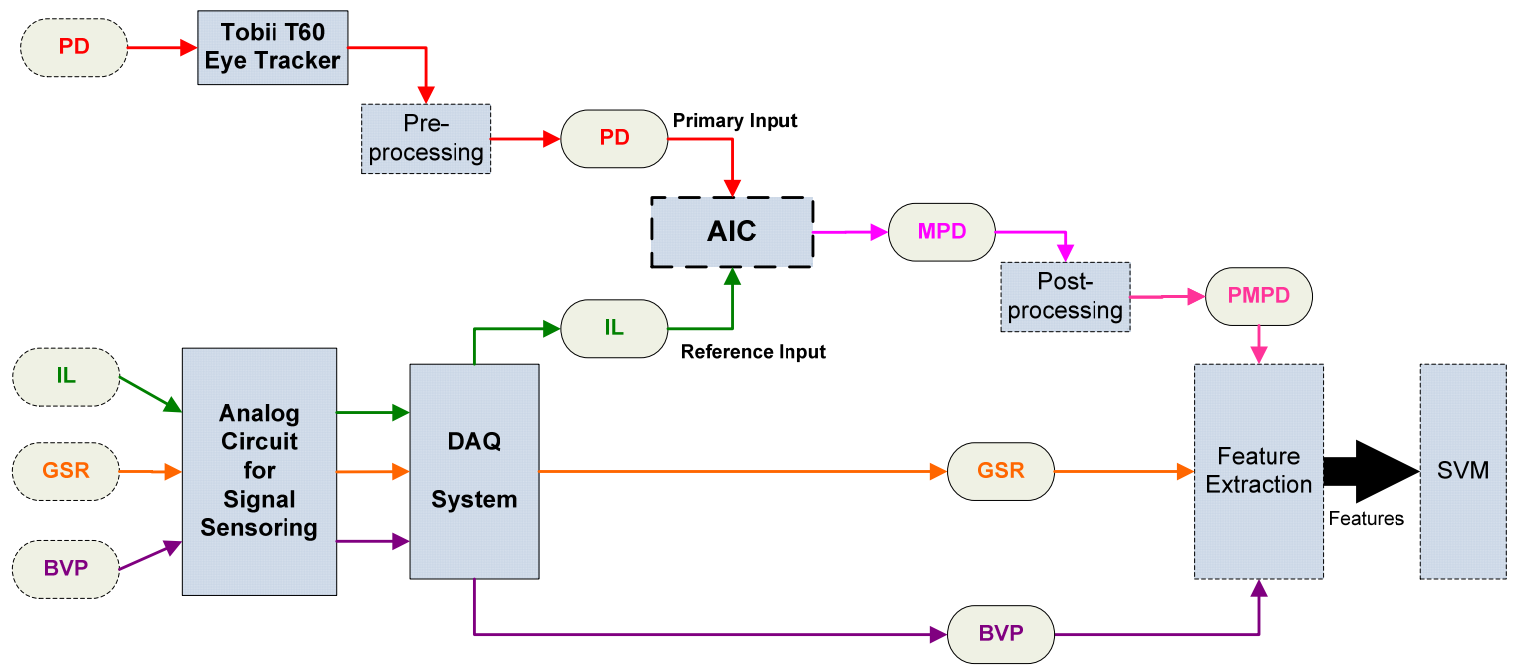

Figure 3-13: Overview of this research methodology

Although the PD and IL signals are recorded separately, after pre-processing, the PD is fed to the AIC together with IL as the primary input and reference input respectively. The output of AIC (MPD) is further processed by a post-processing step to generate the PMPD signal, which is used to extract one feature indicator for the stress classification through SVM. Simultaneously, the GSR and BVP signals are sensed and conditioned with analog circuits and digitized by the DAQ unit. Features derived from these two physiological signals are analyzed by the SVM as well to provide a stress recognition performance comparison. 


\subsection{Summary}

While the concepts of Affective Computing were proposed more than one decade ago, the development of affect sensing systems through pupil diameter monitoring is still an open research challenge. This may be due to the well known PLR, which is the dominant factor for the pupil size variation. This dissertation proposes to use an AIC system with its HITV adaptive algorithm for the PLR interference cancelling, which has been described in detail at the beginning of this chapter. The processing and feature extraction methods applied to the PD, GSR and BVP signals were outlined in the corresponding sections. The later sections of this chapter described the data normalization stages applied to the features derived from the PD, BVP and GSR signals and the machine learning approach (SVM) chosen for the classification of those features. Similarly, the use of ROC curves to assess the discriminating power of each individual feature was proposed. The last section of the chapter presented a figure that summarizes the overall research process used for this study. 


\section{CHAPTER 4 \\ EXPERIMENT DESIGN}

The overall goal of this research is to develop a signal processing and machine learning method that can distinguish an affective state of "stress" from one of "relaxation", in a computer user, by the analysis of simultaneously measured PD and IL signals from the subject. The discrimination power of the feature obtained from the processed pupil diameter signal will be compared with 9 other features derived from GSR and BVP signals. To that end, an experimental setup and a corresponding protocol were defined and implemented for the project to:

(i) Provide an appropriate stimulus, capable of eliciting stress in the subjects participating in the experiment;

(ii) Provide appropriate illumination changes, so that a pupil light reflex will be evoked in the subjects, at known intervals;

(iii) Provide appropriate coordination of all the software components involved in the experimental process;

(iv) Record the PD, IL, GSR and BVP signals with all the necessary time markers.

The complete implementation of the experimental environment together with the coordinated software and hardware components are described in the following sections. 


\subsection{Software Development}

In order to observe the changes of the physiological signals (PD, BVP and GSR) and their correlation to sympathetic activation caused by stress, it was necessary to elicit stress in the experimental subjects during controlled intervals.

In this study, the subjects were instructed automatically by the computer to participant in a Stroop Color-Word Interference Test [68], implemented as a Flash program [69], which was used to elicit mild mental stress in the subjects at known times. The approval of the FIU Internal Review Board (IRB) for experimentation with human subjects according to this protocol was sought and obtained (Appendix A). The efficiency of this stress elicitation method has been previously established by Renaud and Blondin[70]. The implementation of this stimulus program is described in the following section. Before the actual Stroop Test started, each subject was required to watch 30 emotionally-neutral still pictures to set a baseline of relaxation. Each of these pictures was displayed for 15 seconds, which means the preliminary relaxation period is about seven and a half minutes in total.

\subsubsection{Computer-Based Stroop Test}

Since 1935, the Stroop Color-Word Interference Test has been utilized as a psychological or cognitive stressor to induce emotional responses and heightened levels of physiological, (especially autonomic) reactivity[71]. In this study, a programmed computer-based interacting environment [69] was used to let the subjects experience a similar effect. During the test, a word presented to the subject designates a color that may or not match the font of the word. The subjects are instructed to select from the five 
buttons shown on the screen the one that identifies the font color of the word presented. Typical examples of this test interface are shown on the Figure 4-1 and Figure 4-2

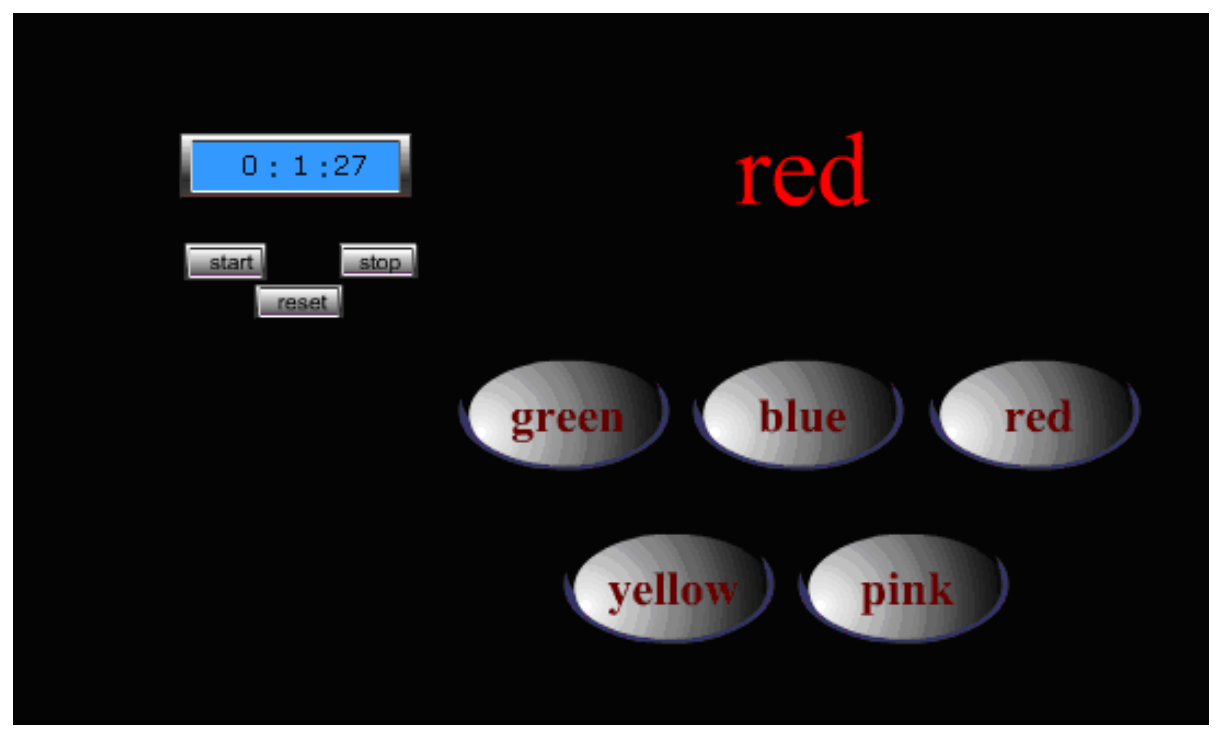

Figure 4-1: Sample Stroop Test interface (Congruent section, the font color of "red" is red)

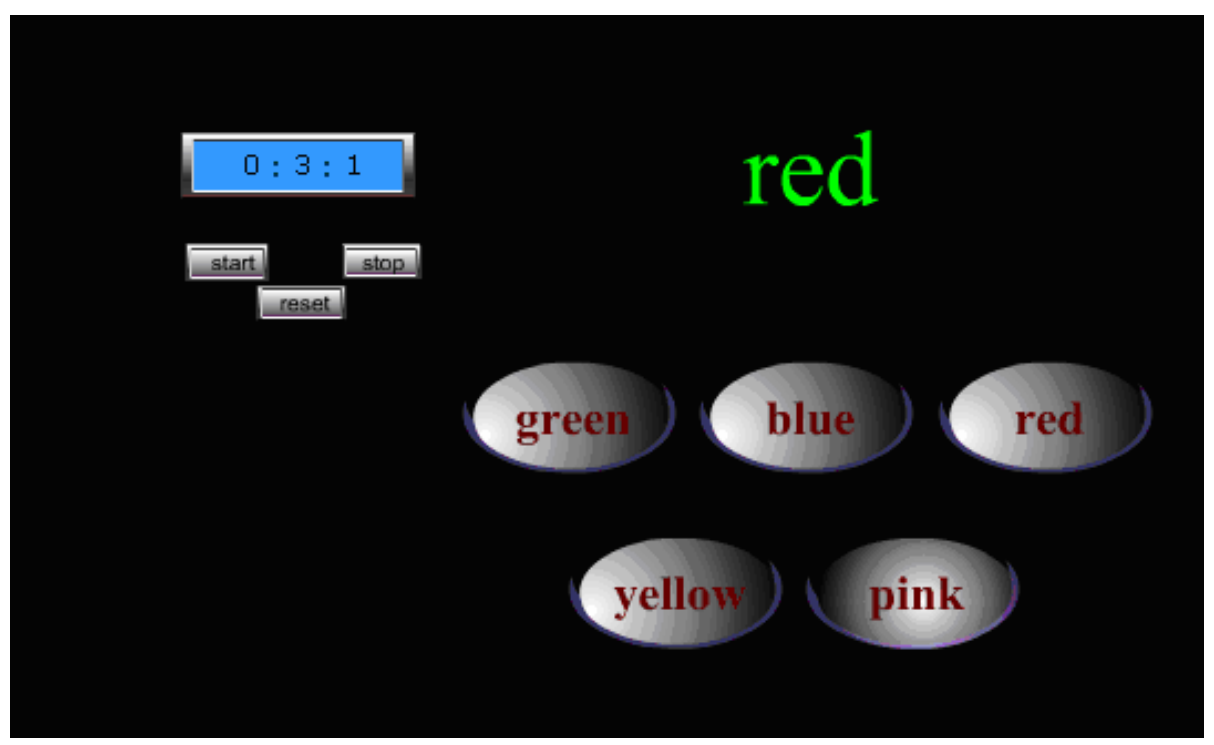

Figure 4-2: Sample Stroop Test interface (Incongruent section, the font color of "red" is green)

Figure 4.1 shows a Congruent Stroop ("C") word presentation, in which the font color and the meaning of the word shown match. In contrast, Figure 4-2 is an example of Incongruent Stroop ("IC") word presentation, where the font color and the meaning of the 
word are different, causing an internal "interference" in the subject's attempt to identify the font color.

To avoid subject's adaptation through repeated clicks, the position of the five answer button was varied for each IC section, which was meant to enhance the stress elicitation (the change in the positions of the buttons can be appreciated in Figure 4-3 \& Figure 4-4 )

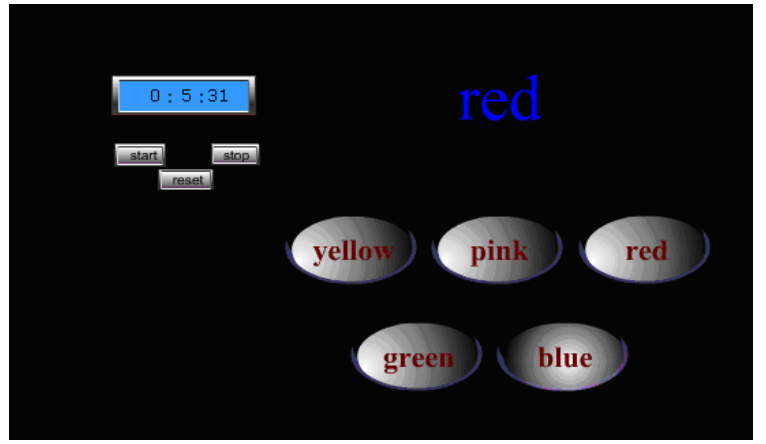

Figure 4-3: Sample Stroop Test interface (IC2 with changed button position)

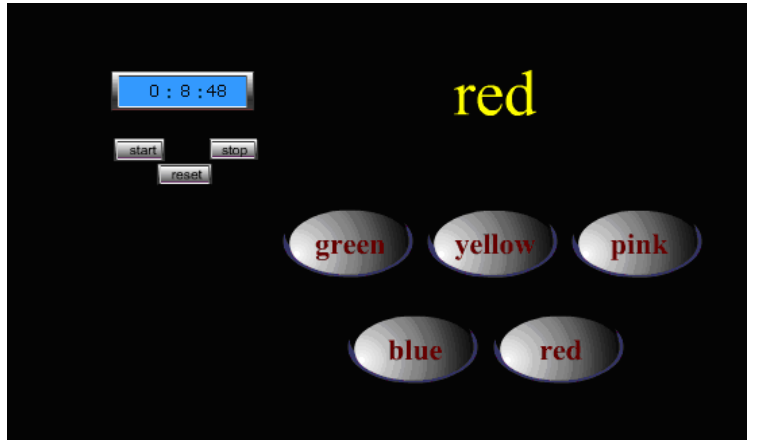

Figure 4-4: Sample Stroop Test interface (IC3 with changed button position)

\subsubsection{Stimuli Schedule in Test}

In this dissertation, the Stroop Color-Word Interference Test was implemented with Macromedia Flash ${ }^{\circledR}$. The complete experiment sequence (Figure 4-5) is composed of three consecutive sections. In each section, there were four segments, including:

$\checkmark$ "IS" - the Introductory Segment to let the subject get used to the task environment, in order to establish an appropriate initial level for his/her psychological state, according to the law of initial values (LIV)[72];

$\checkmark$ "C" - the Congruent segment, comprising 45 Stroop Congruent word presentations (font color matches the meaning of the word), which are not expected to elicit significant stress in the subject; 
$\checkmark$ "IC" - the Incongruent segment, in which the font color and the meaning of the 30 words presented differ, which is expected to induce stress in the subject;

$\checkmark$ "RS" - a Resting Segment to let the subject return to a baseline affective state.

The Incongruent Stroop segments (IC) were expected to elicit mild mental stress in the subject, according to previous research found in the psychophysiological literature [70]. In contrast, the congruent Stroop segments (C) were expected to allow the subject to continue in a "relaxed" state.

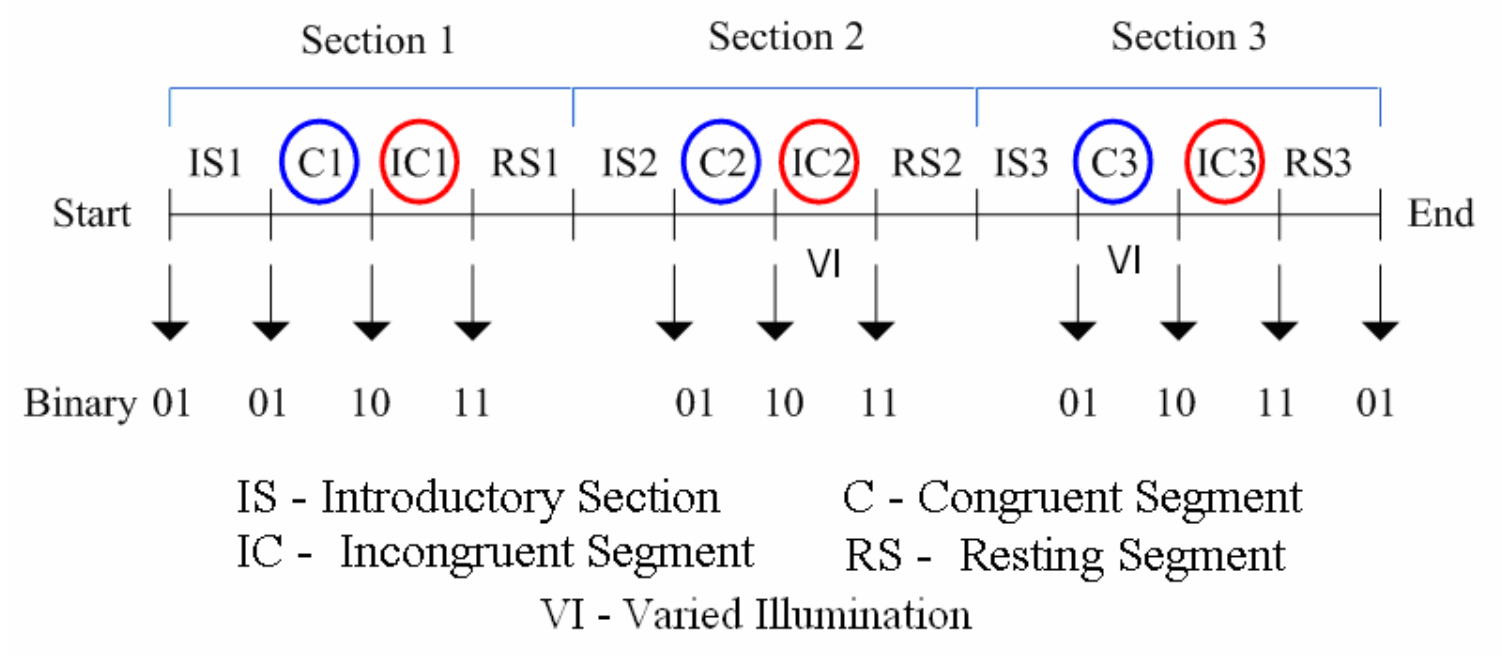

Figure 4-5: Complete experiment sequence

Since the study aimed the analysis of the physiological signals and their changes from a state of "relaxation" to a state of "stress", the boundaries of the C and IC segments need to be marked in the data. For this purpose, bursts of sinusoidal tones were output through the sound system of the computer following a binary encoding $(01,10$ or 11$)$ whenever one of the C, IC or RS (respectively) segments started. These sinusoidal bursts, recorded on two additional channel of the data acquisition system, served as time-stamps to the physiological signals recorded at those critical instants. The binary numbers shown at the bottom of Figure 4-5 encode the patterns of generation of the audio bursts used in 
the system to time-stamp the physiological signals. " 01 " represents a burst in the left channel audio signal, "10" represents a burst in the right channel and "11" represents simultaneous bursts in both channels.

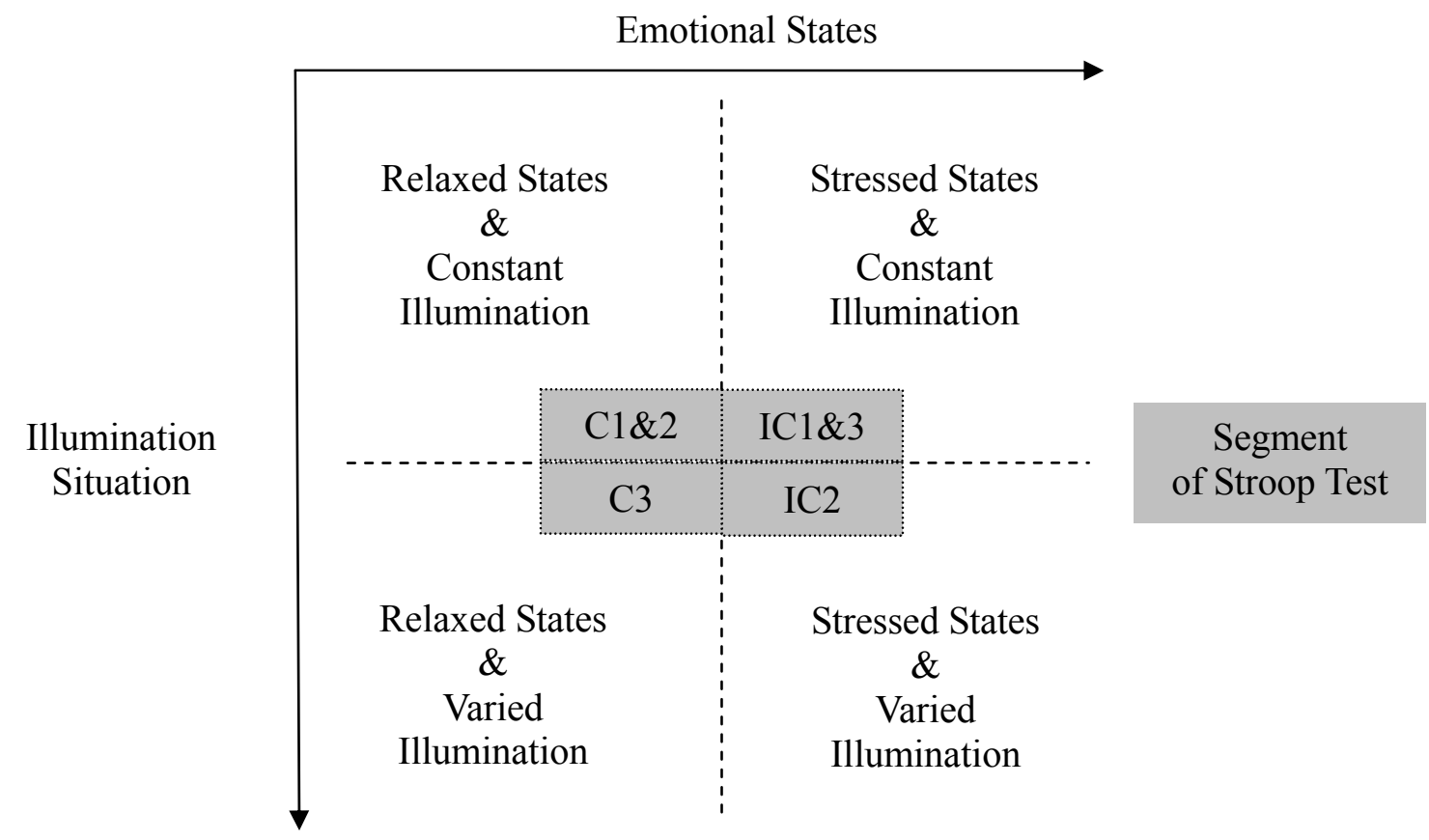

Figure 4-6: Four kinds of experimental situation

A particular aim of this study was to investigate the potential use of the pupil diameter signal for affective sensing, even in presence of changes in the light intensity of the environment surrounding the subject. Therefore, there are 4 kinds of situation in the experiment designed, according to the two states of the affective ("stress" vs. "relaxed") and illumination (constant vs. varied) conditions affecting the subject. These four situations and the experimental segments in which they were implemented are diagrammed in Figure 4-6, above. The luminance intensity remains constant except in the segments IC2 and C3, where the illumination will be increased in a transient fashion. 


\subsection{Hardware Setup Design}

The goal of the systems designed in this research is to provide a continuous digital signal recording of the physiological variables monitored for analysis. In order to record the affective response of the PD,GSR and BVP that take place when stress is elicited in the subject, a hardware system was integrated, in order to:

(i) Record the physiological signals clearly and accurately;

(ii) Record the signals with all the necessary time markers.

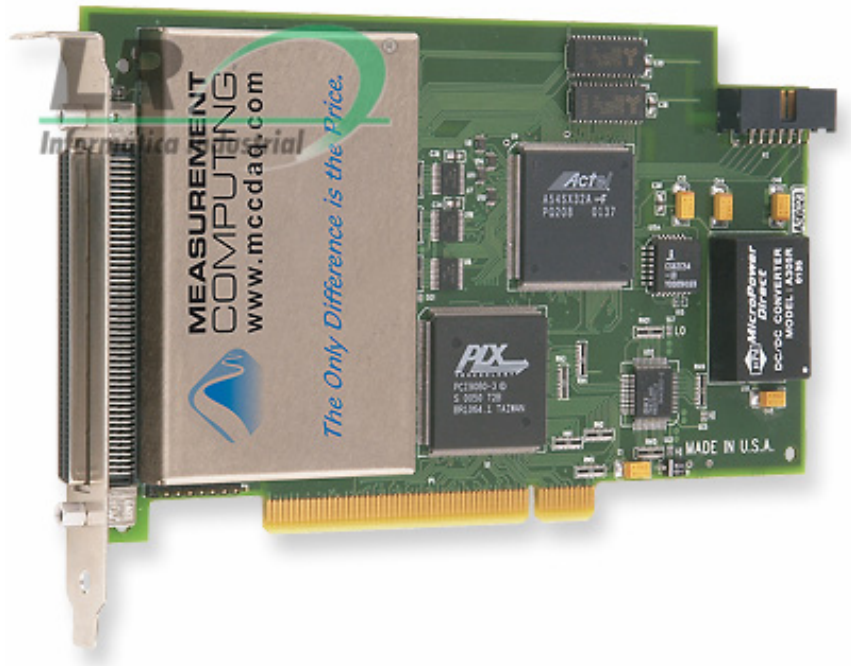

Figure 4-7: DAQ system (PCI-DAS6023 from MCC)

In total, this hardware system is composed of 4 sub-systems, which are "PD Measurement System”, “IL Measurement System”, “GSR Measurement System” and "BVP Measurement System". The GSR, BVP and IL systems provided amplification and conditioning to these signals which were then sampled at the same frequency with a data acquisition (DAQ) System based on the PCI-DAS6023 board from Measurement Computing Co.(MCC). 
All the sensors described in the following paragraphs meet the criteria of providing a continuous recording while also being minimally invasive to the computer user's normal activity. The considerations made in the integration of the required hardware setup are described in the following sections.

\subsubsection{PD Measurement System}

In order to obtain real-time measurements of the diameter of the user's pupils, a desk-mounted eye tracking system (TOBII T60), shown in Figure 4-8, was used to record the pupil diameter signals from both eyes of the subjects, at $60 \mathrm{~Hz}$.

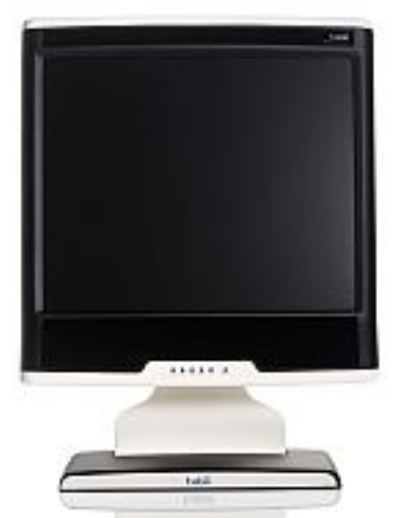

Figure 4-8: PD measurement system device - TOBII T60 eye tracker

A program to control the TOBII system for the continuous acquisition of PD data developed in Microsoft Visual Studio 2005 with the C\# language. This program used the building blocks provided by the TOBII software development kit (SDK). The interface of this program can be shown in the following figure. Generally, there are three steps involved in the process of monitoring the pupil diameter of the computer user with the TOBII system, which are indicted by the circles of the figure. 
(i) Confirm Track Status - to verify that the eye tracker is able to detect both eyes of the subject that and the system is correctly tracking the user's pupils;

(ii) Calibrate -to associate directions of gaze with corresponding points of gaze on the screen. TOBII uses a 9-point calibration procedure, which allows certain level of tolerance for small head movements of the subject;

(iii) Track -start tracking and reporting data, which including the pupil sizes (PD) of both eyes and their validity code.

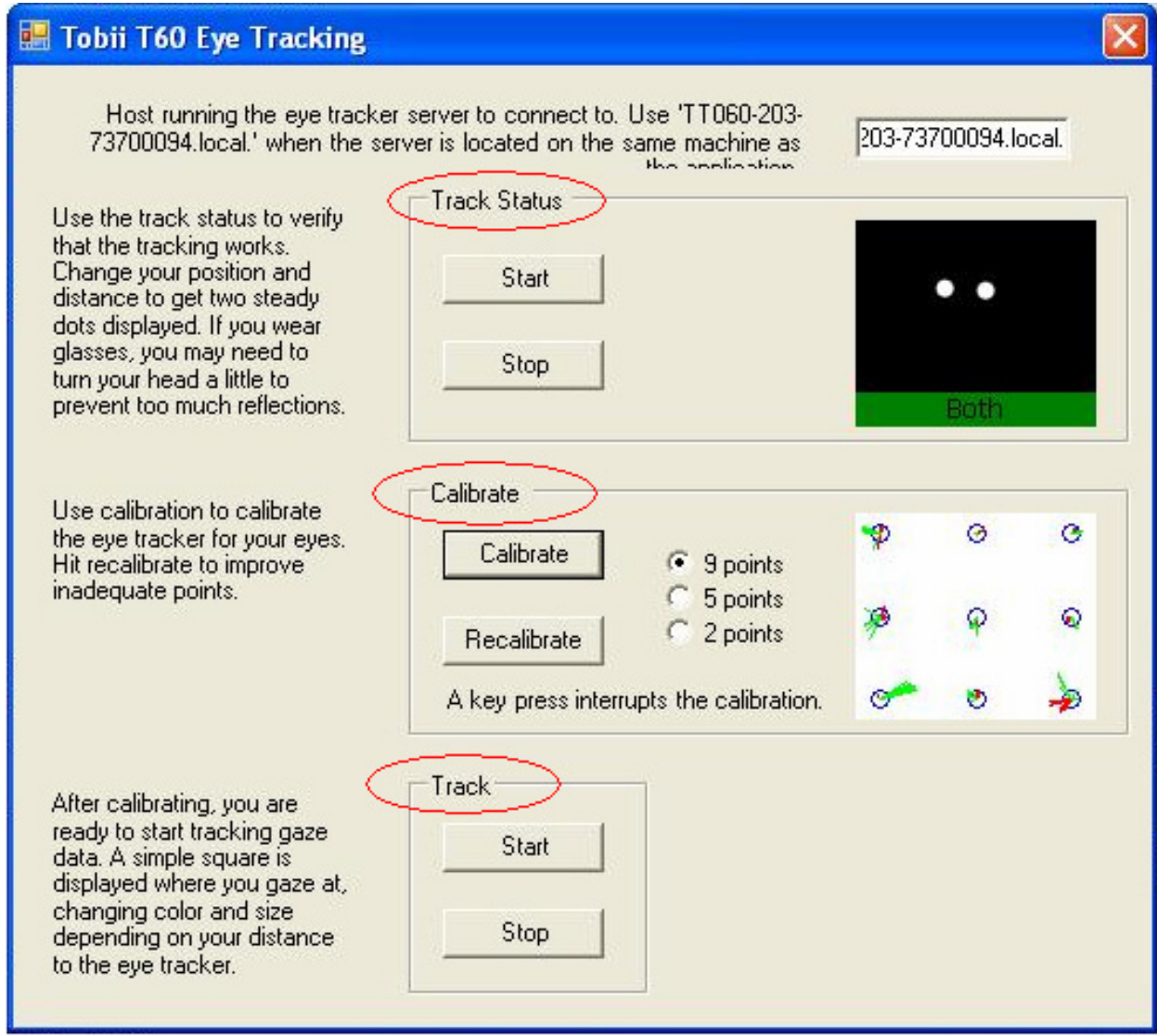

Figure 4-9: TOBII T60 eye tracker interface

Although the PD tracking for both eyes of the subject starts at the beginning of the preliminary relaxation period (displaying emotionally-neutral pictures) the average pupil diameter $((\mathrm{L}+\mathrm{R}) / 2)$ was recorded only during the Stroop Test portion of the 
experiment. This average PD sequence is considered the "Measured PD" signal, which corresponds to the $d(k)$ signal in the adaptive interference canceller (AIC) application diagram (Figure 3-2).

The validity codes reported by the TOBII system for both eyes was also recorded and used to estimate how valid the data are. The validity code takes one of five values for each eye ranging from 0 to 4 , with the interpretations shown in Table 4-1

Table 4-1: Possible values of the validity code from the TOBII system

\begin{tabular}{l|l} 
Code & Description \\
\hline $0-0$ & Data is valid for both eyes. \\
\hline $0-4$ or 4-0 & Data is valid for one eye, and the data for the other is missing. \\
\hline $1-3$ or 3-1 & Data is valid for one eye, and the data for the other is based on estimation. \\
\hline $2-2$ & Data is valid for either one of the eyes, but not sure which one it is. \\
\hline $4-4$ & Data for both eyes are invalid. (Identified as Blinks) \\
\hline
\end{tabular}

As described above, only the recorded PD data with valid code 0-0 will be considered as a successful measurement. The PD samples with a pair of validity code 4 will be recognized as eye blinks, and the other series of combinations will be identified as artifact, which need further processing before its application to the AIC as the primary input.

\subsubsection{IL Measurement System}

To measure the luminance intensity affecting the area around the eyes of the subject, an Illumination (IL) Measurement System (Figure 4-10) is employed. 


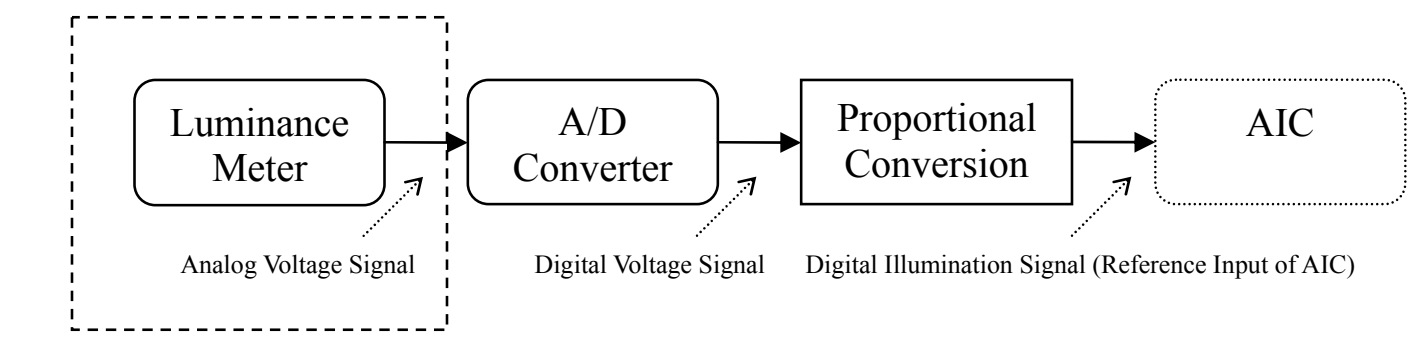

Illumination Measurement System

Figure 4-10: Illumination measurement function

This system is based on the implementation of a luminance meter. A BS500B0F photo-diode (Sharp), the sensor component of the luminance meter circuit (Figure 4-11), was placed on the forehead of the computer user to measure the illumination in the area around his/her eyes.

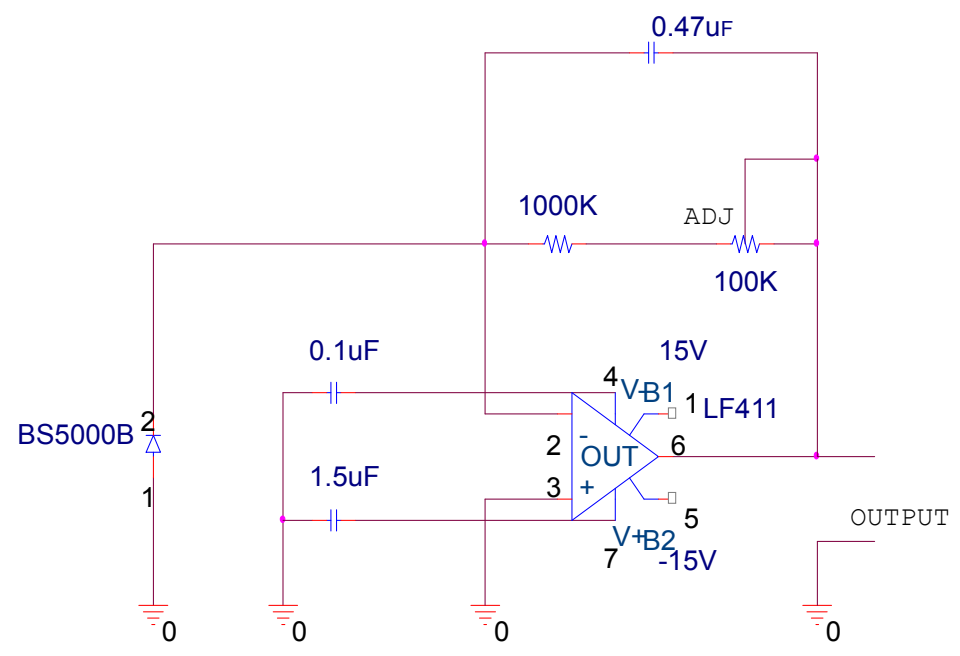

Figure 4-11: Circuit of luminance meter[73]

By using this circuit, changes of luminance can be linearly converted to voltage changes in the analog output signal. The proportional conversion rate is obtained by testing this luminance meter while varying levels of luminance intensity were simultaneously measured with a calibrated meter (Extech Instruments, Mod. 401036). A set of measurement data pairs is listed in Table 4-2 and plotted in Figure 4-12. They 
verify the linear transduction with the conversion factor of about $0.0043 \mathrm{v} / \mathrm{Lux}$ between the two physical quantities.

Table 4-2: Test data of luminance meter

\begin{tabular}{|c|c|}
\hline $\begin{array}{c}\text { Ilumination } \\
\text { ( Lux })\end{array}$ & $\begin{array}{c}\text { Voltage } \\
\text { ( V ) }\end{array}$ \\
\hline 540 & 2.075 \\
\hline 570 & 2.283 \\
\hline 607 & 2.508 \\
\hline 649 & 2.740 \\
\hline 694 & 2.990 \\
\hline 724 & 3.155 \\
\hline 776 & 3.435 \\
\hline 801 & 3.508 \\
\hline 866 & 3.910 \\
\hline
\end{tabular}

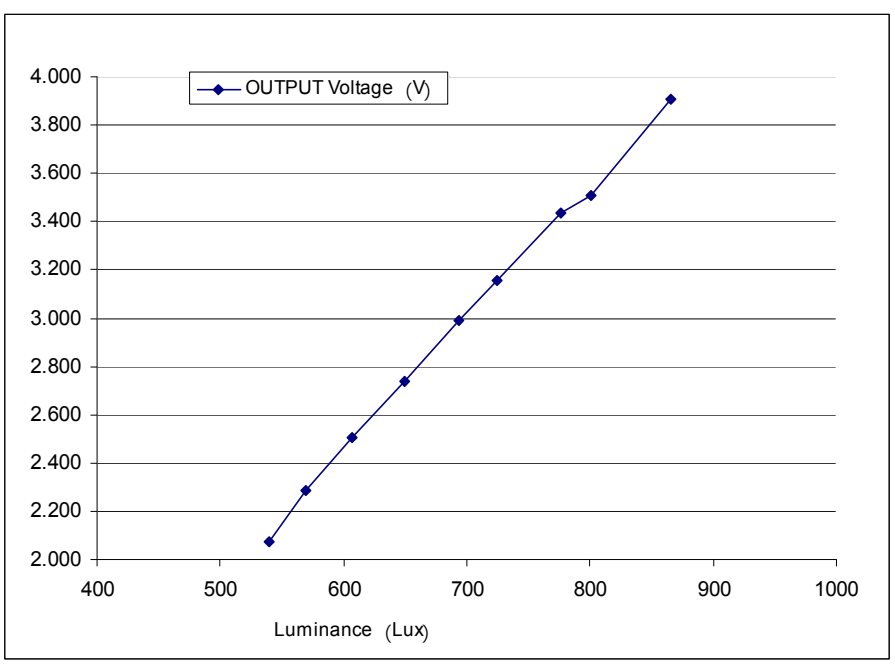

Figure 4-12: Linear relation between luminance and voltage

To obtain the digital signal of illumination in real time, the analog voltage signal recorded by the IL Measurement System was digitized $(360 \mathrm{~Hz})$ by the a DAQ System and proportionally converted to obtain the "Measured IL" signal, $r(k)$, reference input of AIC [49].

\subsubsection{GSR Measurement System}

Generally, there are two methods commonly used for the skin conductivity or galvanic skin response (GSR) measurement. These are is either from the palm of the hand or the sole of the foot. In our study, a GSR2 module (Figure 4-13), by Thought Technology LTD (West Chazy, New York), combined with a "frequency to voltage 
converter" integrated circuit (LM2917N), shown in Figure 4-14, were used to measure the GSR from the hands of subjects.

The molded plastic case of this GSR2 module is ideal to have the hand of the subject rest on it and the two elongated electrodes are large enough to provide appropriate contact with the volar surfaces of the medial and distant phalanges in two of the subject's fingers, as recommended by Dawson, et al [74]. A small current was passed through this pair of electrodes, measuring the conductivity level, which would be converted, to an oscillation frequency and, eventually, to a proportional to voltage.

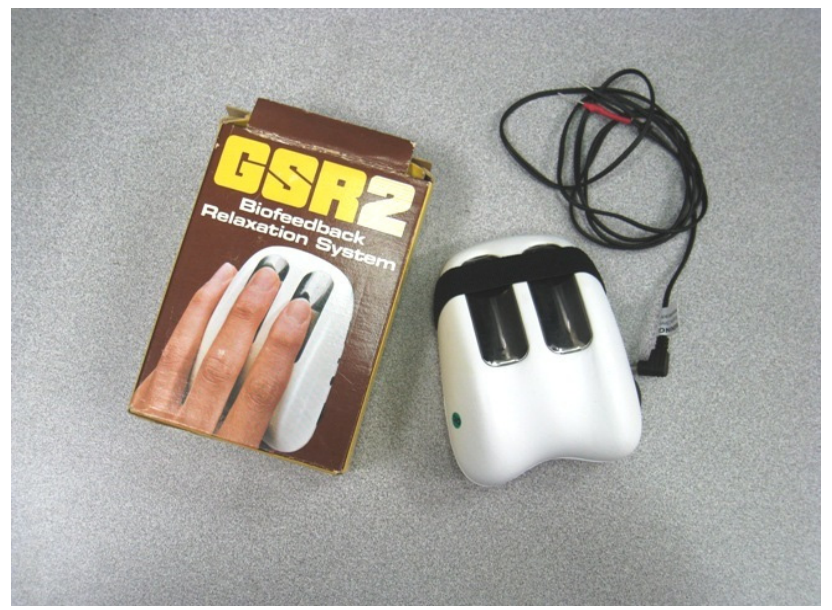

Figure 4-13: GSR2 module for GSR measurement system

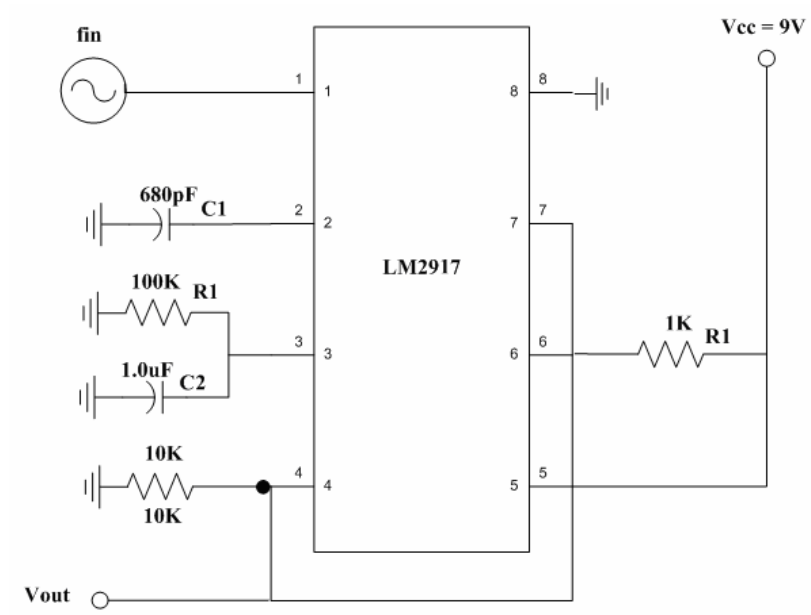

Figure 4-14: Frequency to voltage converter circuit for GSR measurement system 
The output voltage of this frequency-to-voltage converter circuit has a linear relationship with the frequency of the signal at the output of the GSR2 module (Equation 4.1). Vout was then digitized by the DAQ System, at a sampling rate of 360 samples/second.

$$
V_{\text {out }}=V_{c c} \times R_{1} \times C_{1} \times f_{\text {in }}=9 \mathrm{~V} \times 100 \mathrm{~K} \times 680 \mathrm{pF} \times f_{\text {in }}=0.0612 f_{\text {in }}
$$

\subsubsection{BVP Measurement System}

It is known that sympathetic activation causes changes in heart rate, stroke volume and peripheral cardiovascular resistance [75]. All of these responses can be non-invasively sensed by monitoring the amount of blood perfusion in a peripheral region of the body, such as the tip of a finger. In this paper, an infrared finger photoplethysmograph (PPG) (UFI Model 1020), which relies on the light absorption characteristics of blood, was utilized for the measurement of the BVP signal.

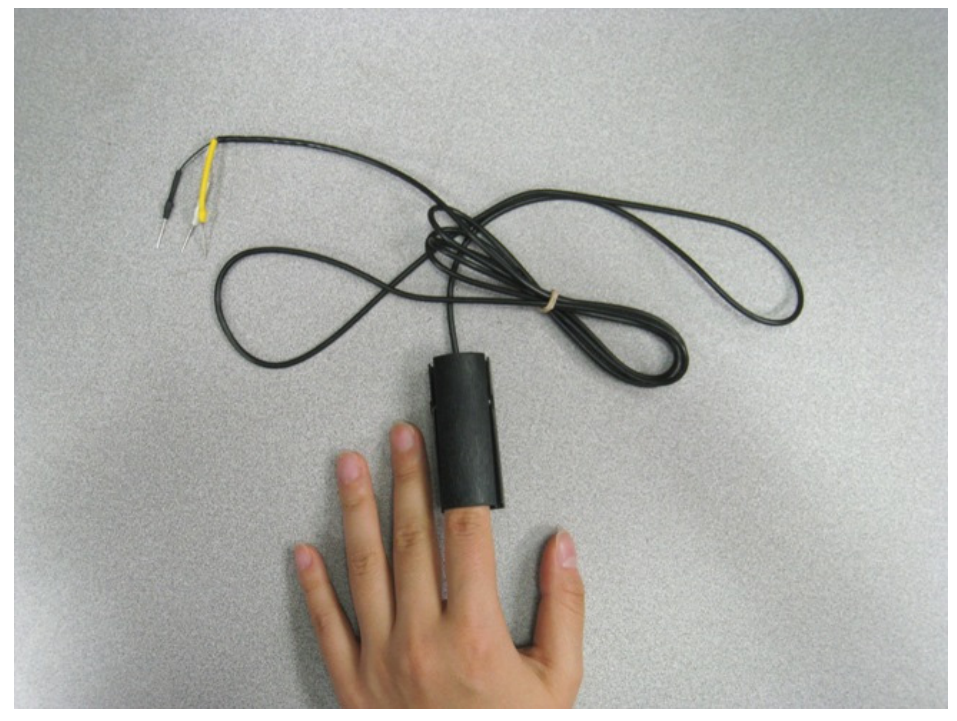

Figure 4-15: BVP sensor -PPG (UFI Model 1020) 
The principle of operation of the PPG is based on the projection of infrared light on the subject's fingertip and the measurement of its reflection. Along its trajectory from transmitter to receiver, the infrared radiation is attenuated, depending on the volume of blood entering the tissue under observation, due to reflection scattering and absorption. Therefore, the measurement of the reflected infrared light will indicate the blood volume variations.

The output of this PPG sensor was amplified about 100 times and passed through a 2nd order low pass filter to minimize the noise from fluorescent lamps that may pollute the signal. The electronic circuit designed for the amplification and filtering of the BVP signal is shown in Figure 4-16. The output voltage from this circuit was digitized by the data acquisition (DAQ) unit (PCI-DAS6023 board from Measurement Computing Co.), using a sampling rate of 360 samples/second.

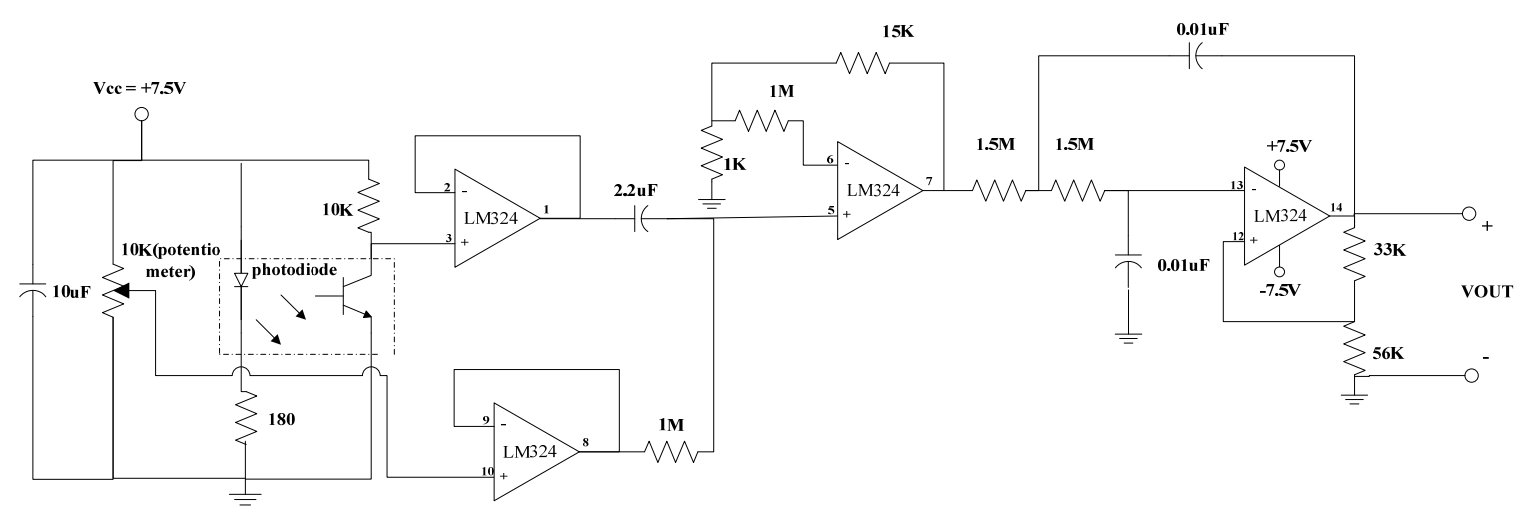

Figure 4-16: The circuit design for the BVP measurement system

\subsubsection{Synchronization of the Recording of All Signals Measured}

In order to compare the identification of variations derived from the monitored pupil diameter (PD) signal with the concurrent changes in two other physiological signals, 
GSR and BVP, as multiple expressions of a single change in affective state, it is necessary to synchronize the independent recordings obtained from the data acquisition (DAQ) System (PCI-DAS6023 board from Measurement Computing Co.), including IL, BVP, GSR and audio markers, and the PD values of both eyes together with their validity code collected from the TOBII T60 eye tracking system. To achieve this required synchronization, the "Start" and "Stop" buttons in the "Track" section of the TOBII control program (circled buttons in Figure 4-17) were programmed to begin and end the recording of the subject's PD values, and simultaneously start and stop the collection of samples of the other signals (GSR, BVP, IL and audio markers) from the DAQ.

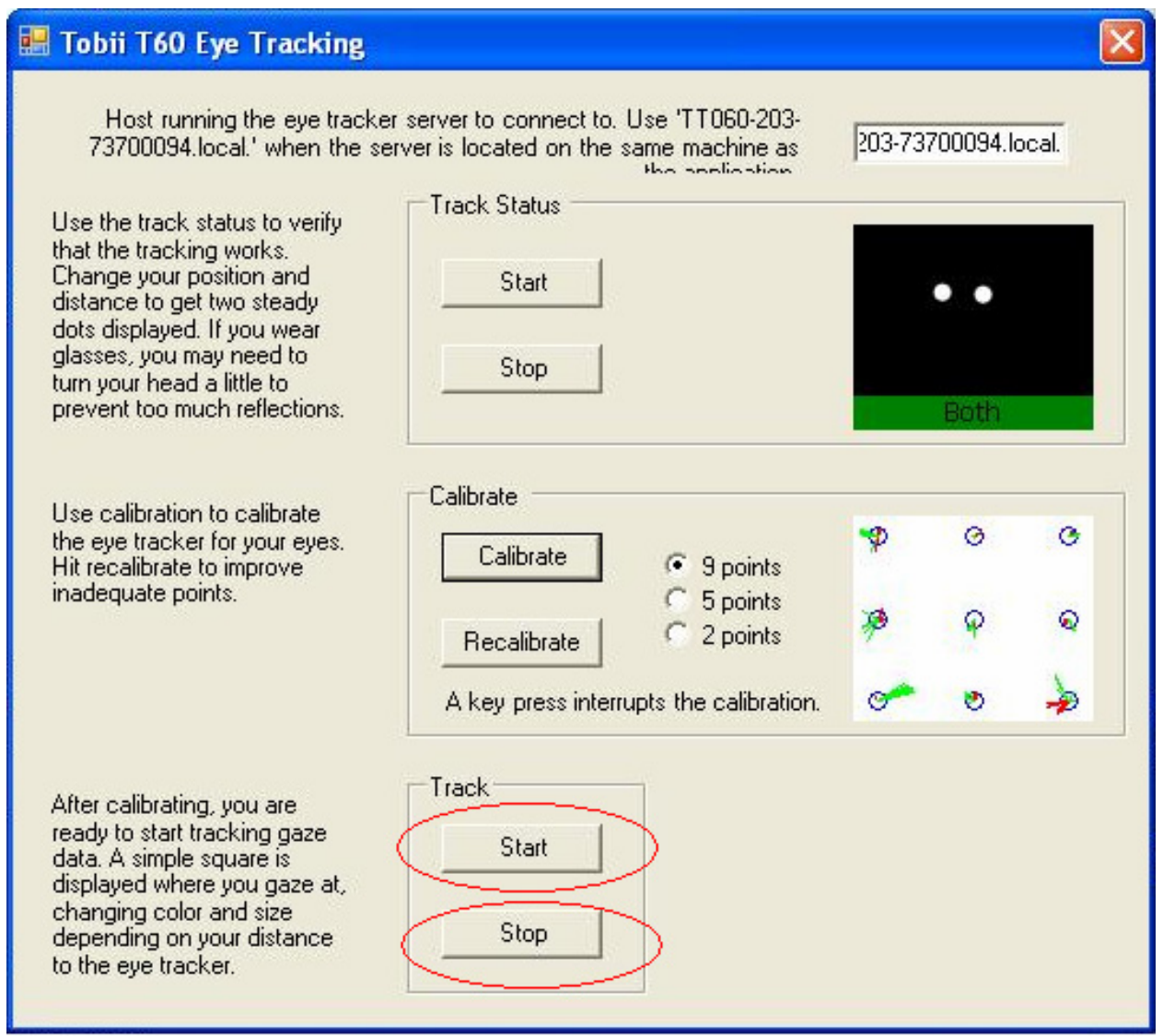

Figure 4-17: "Start" and "Stop" button in the "Track" section of TOBII control program 


\subsubsection{Overall Instrumental Setup}

The complete instrumental setup developed for the research described here is illustrated in Figure 4-18.

This figure shows that the visual stimuli for the subject (Stroop word presentations), described in section 4.1, were displayed on the TOBII T60 eye tracker monitor. The program developed for the eye tracking system allows the extraction of selected variables (in this case the PD of both eyes and their validity code) to a file at a frequency of $60 \mathrm{~Hz}$, which, in turn, can be read into MATLAB.

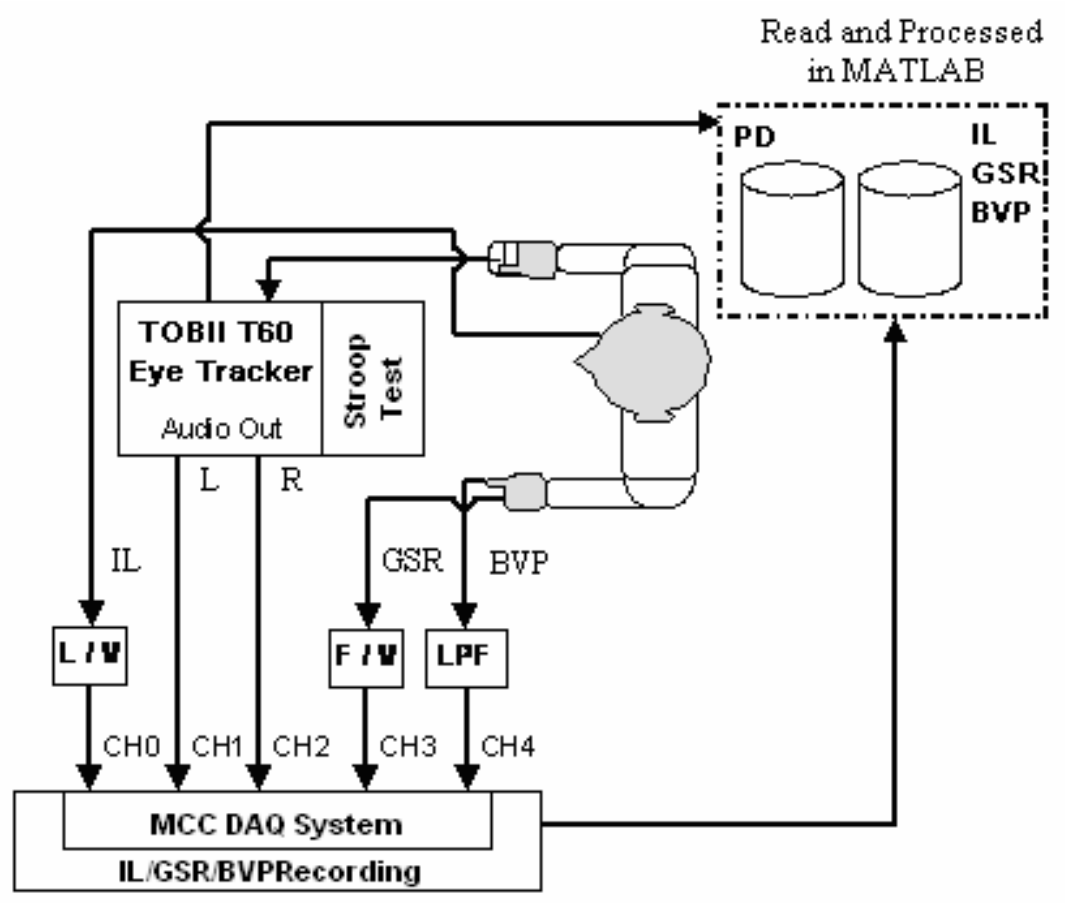

Figure 4-18: Complete instrumental setup

While performing the Stroop Test, the subject has the GSR and BVP sensors attached to his/her left hand and the IL sensor on his/her forehead, above the eyes. All these three signals, together with the left and right audio output (to provide the 
corresponding time stamping in the experiment) are recorded and converted to a MATLAB ${ }^{\circledR}$-readable data file directly at rate 360 samples/second, using a multi-channel MCC DAQ system (PCI-DAS6023 board). Later, these data were down-sampled by six, to share the same sampling rate at 60 samples/second for all measured signals (PD, IL, GSR, BVP and audio output).

The following Figure 4-19 shows a participant with the IL, GSR and BVP sensors attached to him and the TOBII T60 eye tracker set up to monitor his pupil diameter during the experiment.

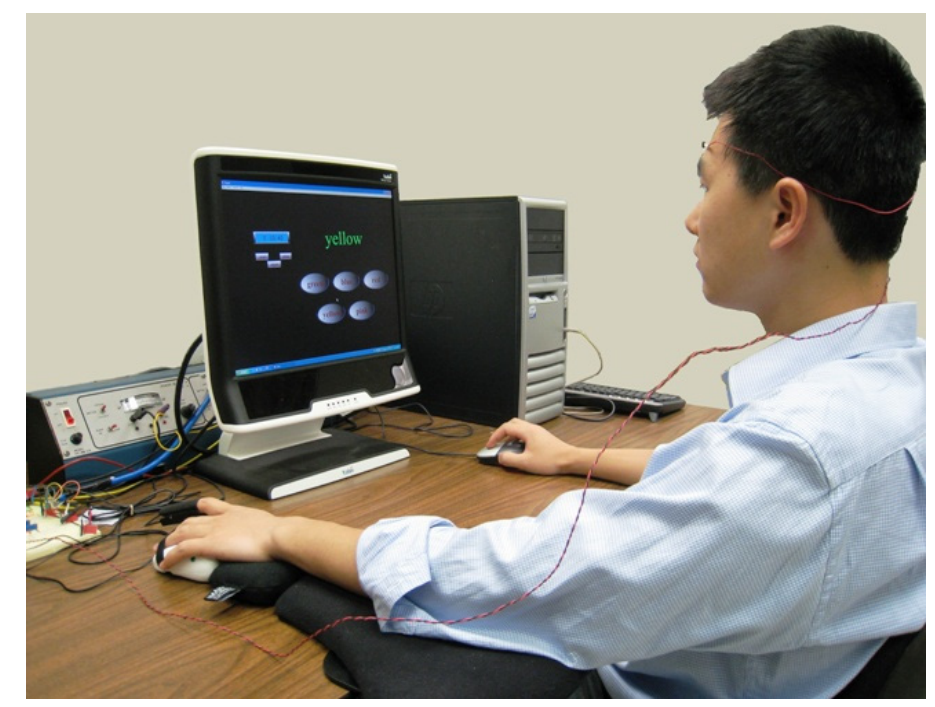

Figure 4-19: An example of participant in the experiment

Figure 4-20 shows an example of the four signals recorded from a subject through the complete experimental session, once all signals have been synchronized (at a sampling rate of 60 samples/second, after down-sampling the GSR, BVP and IL signals). 


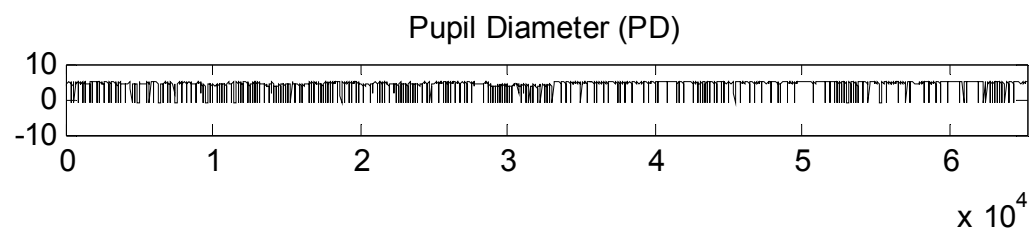

Illumination Intensity Level (IL)

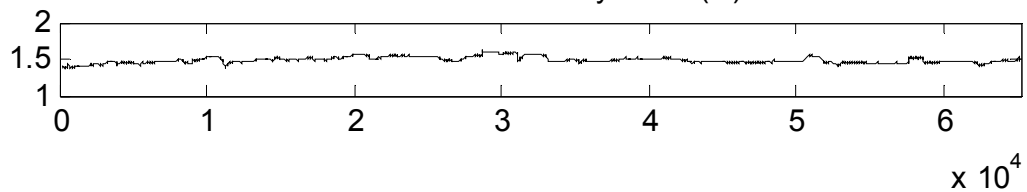

Galvanic Skin Response (GSR)

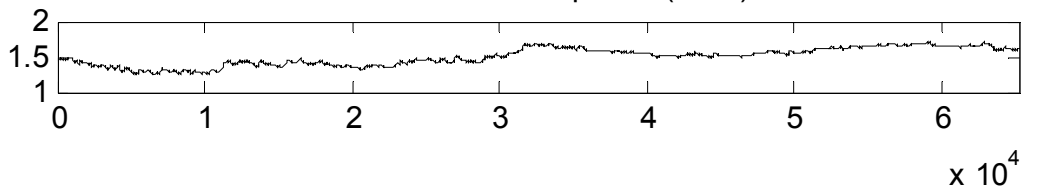

Blood Volume Pulse (BVP)

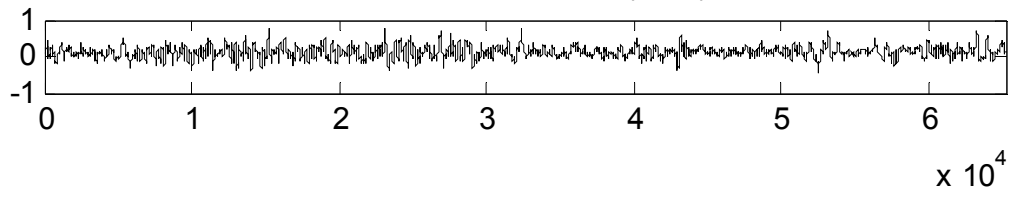

Figure 4-20: PD, IL, GSR and BVP signals in a complete experimental session

\subsection{Experimental Procedure}

In the experiments performed for this study, the participant was asked to remain seated in front of the TOBII screen, interacting with the Stroop Test program for about 30 minutes, while wearing a head band with the photo-diode, the GSR sensor on the middle and ring fingers and the photoplethysmograph on the index finger. During that time, all the normal lights in the room were kept $\mathrm{ON}$, but an additional level of illumination provided by a desk lamp placed above the eye level of the subject was switched ON intermittently, by sliding a dimmer abruptly from its minimum position to its maximum position, and vice versa. This was done to repeatedly introduce intervals of higher illumination in the experiment, which would trigger the pupillary light reflex. 


\subsection{Pool of Experimental Subjects}

Thirty individuals (sixteen female, and fourteen male) volunteered to participate in the experiment. Their age ranged from 24 to 34 years. They were from diverse professional and ethnic backgrounds. Due to the nature of the tasks, the following considerations were made when choosing the participants:

$\checkmark$ Participants had to be fluent in English (to avoid difficulty in understanding the instructions for the computer game);

$\checkmark \quad$ Participants should have good general health (no hearing or sensing problems);

$\checkmark$ Participants should not wear glasses, as this may disrupt the operation of the eye gaze tracker (contact lenses were OK);

$\checkmark$ Participants should be familiar with computers and be experienced in the operation of the keyboard and the mouse.

All of the participants were right-handed. Participants were solicited through phone and emails around the Florida International University area.

\subsection{Summary}

This chapter has described the integrated software and hardware development of a custom instrumental setup capable of providing reliable stimulation to elicit stress in the experiment, record the PD and IL signals for analysis, and monitor other two physiological signals (GSR and BVP), which are used to provide comparison of the stress recognition performance. The experimental procedure and pool of subjects were also described. 


\section{CHAPTER 5}

\section{RESULTS \& DISSCUSSION}

As mentioned in Chapter 3, the PD, GSR and BVP signals collected during each Congruent (C) Stroop segment and each Incongruent (IC) Stroop segment are processed with their own signal processing algorithms to obtain a set of representative features, which could be analyzed by a SVM classification system to recognize the presence or absence of stress in the segment.

The results of each processing and feature extraction step are illustrated in this chapter. In addition, the outcome of the SVM training based on the features of these three physiological signals is described to indicate the correlation between a set of raw data and the internal stress state in each game section. The advantage of affective sensing based on the PD signal, in contrast with the use of other physiological signals (GSR \& BVP) is discussed by the comparison of the corresponding SVM classification accuracy rates. Further details of the affective sensing performance through the PD feature are investigated by an additional SVM study. Finally, the individual detection power of each of the 10 features derived from the signals ( 1 from PD, 5 from GSR and 4 from BVP) is explored through its Receiver Operating Characteristic (ROC) curve.

\subsection{Signal Processing Results for the PD Signal}

Generally, the processing procedure for the PD signal is composed of three steps. They are: 
(i) Pre-processing of the "Measured PD" signal to remove the eye blinks and artifact influence on the recorded signal;

(ii) Process PD and IL signals using AIC with HITV adaptive algorithm to remove the interference of PLR and output an MPD signal, which is an estimation of the PAR;

(iii) Post-processing of the MPD signal to generate the PMPD signal, in which the pupil diameter increases due to sympathetic activation during the IC segments in the experiment are particularly emphasized.

The processing results of each step are illustrated in the following sections. Subsequently, the capability for identification of "stress" segments from the PMPD and PD signal is discussed as well.

\subsubsection{Pre-processing of PD}

In this research, the PD signal is monitored by a TOBII T60 eye tracker, which is used for recording the pupil diameter and the validity codes for both eyes throughout the experiment, at the frequency of $60 \mathrm{~Hz}$ (each of the 4 signals).

An example from one of the 30 subjects who participated in the study (subject 13) is shown in Figure 5-1, below. 


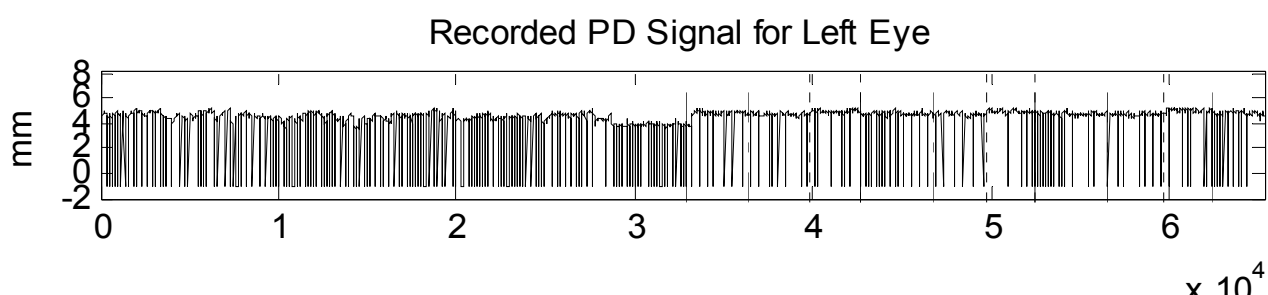

Recorded Validity Code for Left Eye
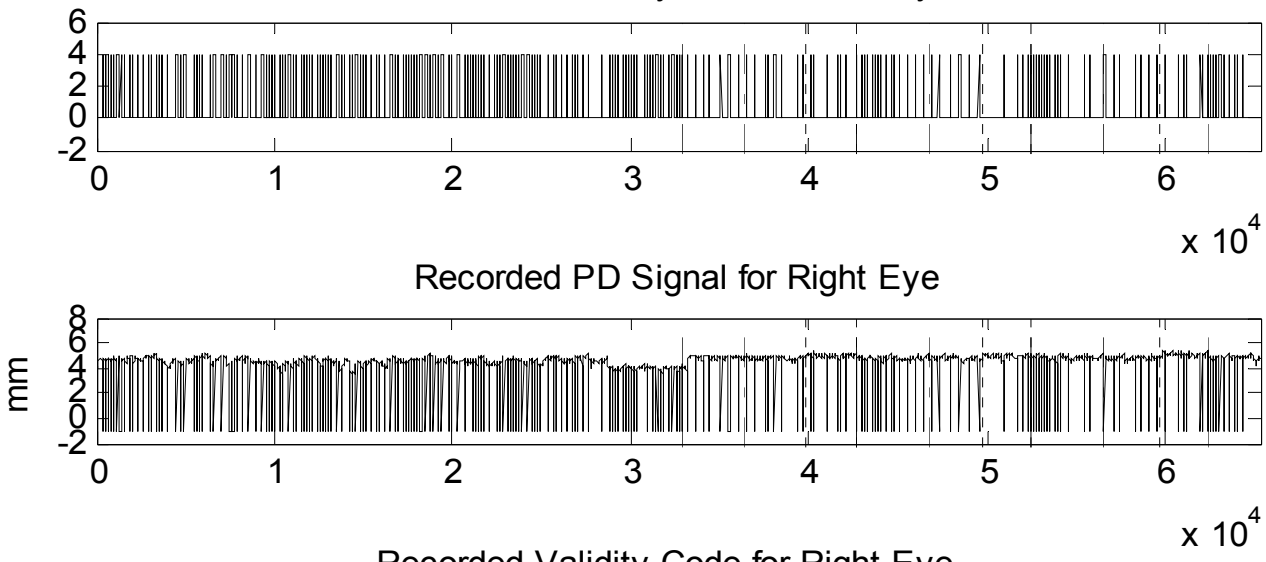

Recorded Validity Code for Right Eye

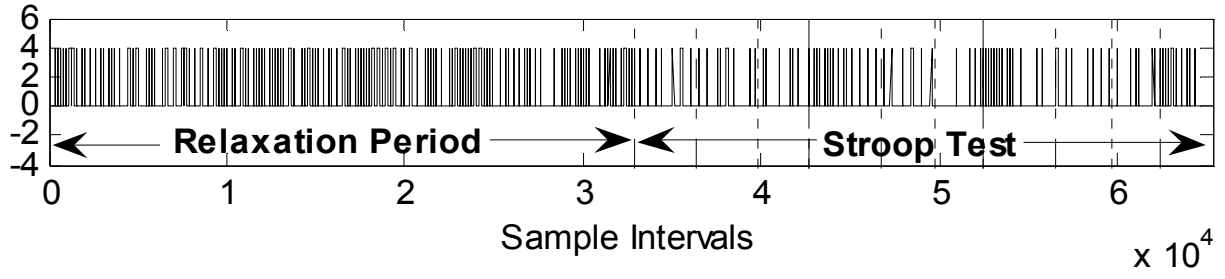

Figure 5-1: PD signals and validity codes of both eyes recorded by the TOBII system

Although the PD signals and corresponding validity codes of both eyes were recorded throughout the experiment, only those parts measured during the Stroop segments (C and IC) are of interest. Therefore, all these signals were separated in a shorter record, which encompassed from 1800 samples (30 seconds) before the beginning of the first Stroop segment (C1) to the end of the last Stroop segment (IC3), according to the recorded audio time markers. The average of the selected portion from PD signals of both eyes was calculated as the "Measured PD" signal in Figure 3-11. Before its application to the AIC, this signal is pre-processed by a blink \& artifact-removal algorithm implemented in MATLAB, which is able to: 
(i) Detect the interruptions due to eye blinks and artifact (identified as a pair of validity codes with at least one value lager than “ 0 ”);

(ii) Compensate the missing data by linear interpolation;

(iii) Filter out the blink responses through a low pass, 512th order FIR filter designed for a cutoff frequency $0.13 \mathrm{~Hz}$;

(iv) Shift forward the FIR output by 256 sampling intervals to maintain the time alignment of the resulting pre-processed signal.
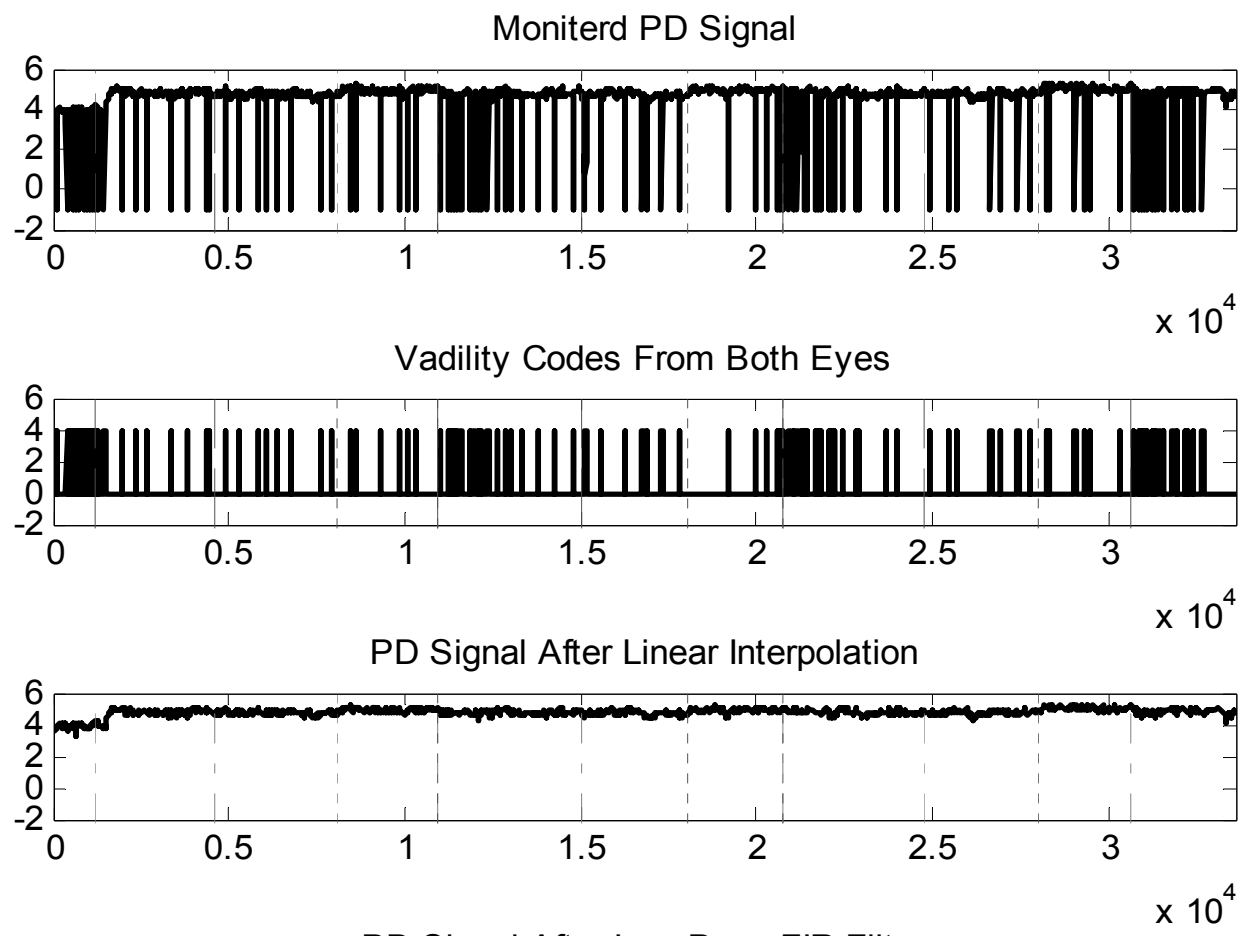

PD Signal After Low Pass FIR Filter

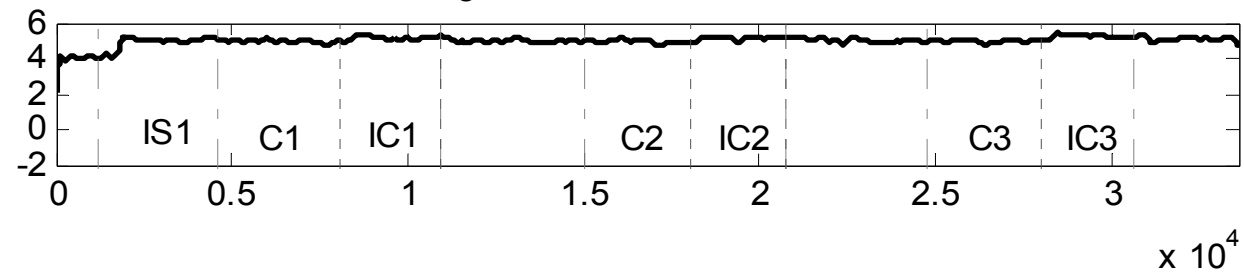

Figure 5-2: PD signal before ("Measured PD") \& after blink-removal

Figure 5-2 shows the left shifted (256 samples) FIR results of the blink \& artifact-removal process (bottom pane), on a "Measured PD" signal (top pane), after 
linear interpolation of the lost values (third pane) based on the validity code from both eyes.

\subsubsection{AIC Implementation using the HITV Algorithm}

The second step of the PD signal processing is implemented by an AIC system programmed in MATLAB, for the purpose of removing the effect of the PLR on the pupil diameter, as recorded. This AIC is set up as follows:

$\checkmark$ Primary input $d(k)$ : the PD signal obtained after blink-removal;

$\checkmark$ Reference input $r(k)$ : the simultaneously measured IL signal, which is down-sampled from $360 \mathrm{~Hz}$ to $60 \mathrm{~Hz}$ to share the same sampling rate with the PD signal;

$\checkmark$ ATF setting: with 120 weights and adapted by the HITV algorithm;

$\checkmark$ HITV algorithm setting: with the parameters set as $\eta=0.001, \varepsilon_{g}=2.0$ and a time-varying parameter $\rho$, changed according to the IL value to enable the AIC system to have a quicker response when there is a sudden increase in IL.

The output of the AIC, the MPD signal, is shown in the bottom plot of Figure 5-3. This signal is further processed as described below to become a useful indicator of pupil response to the subject's affective variation. 


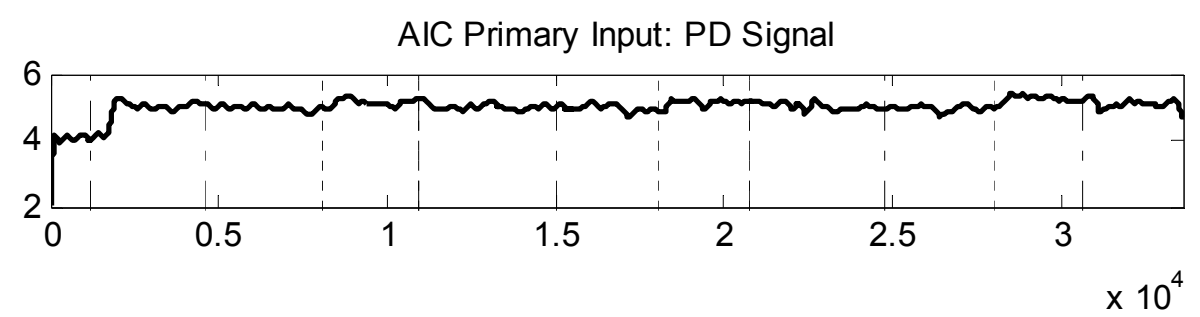

AIC Reference Input: IL Signal
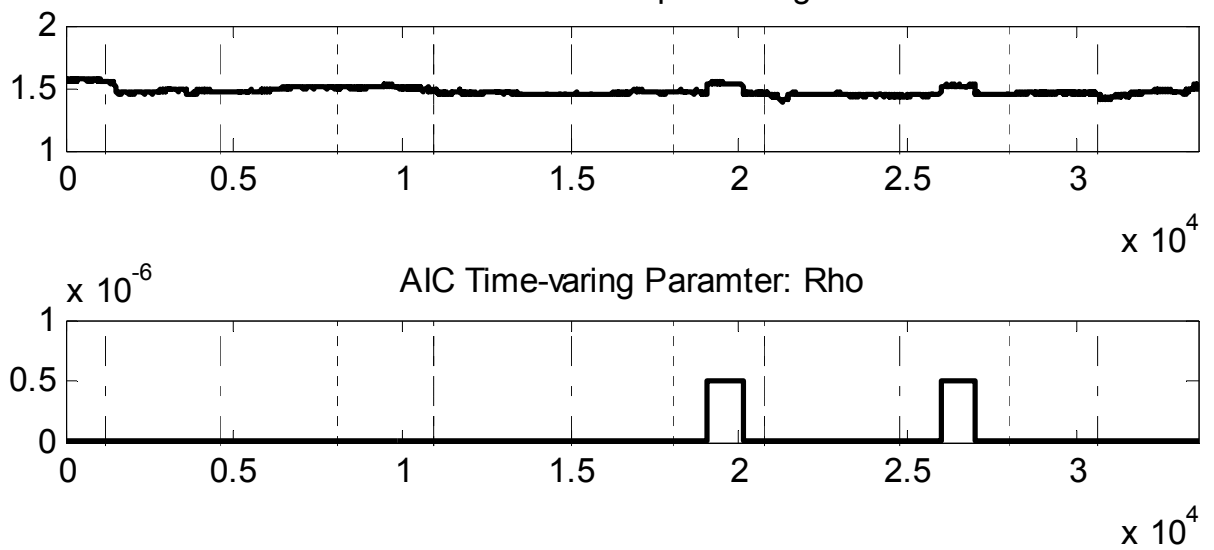

AIC Oouput: MPD Signal

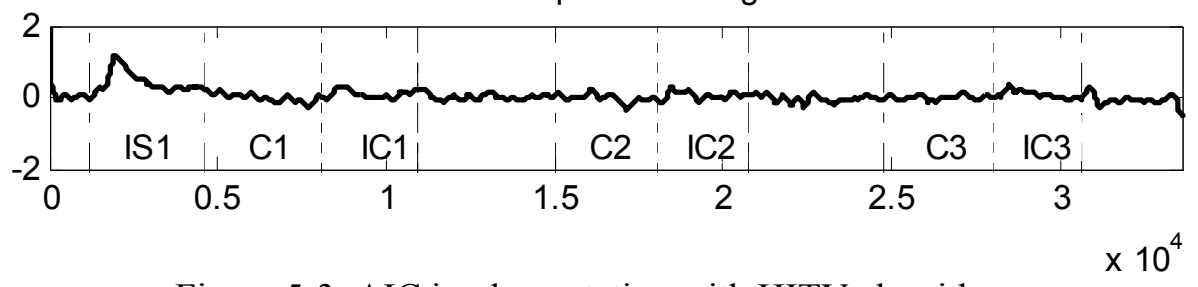

Figure 5-3: AIC implementation with HITV algorithm

\subsubsection{Post-processing of PMPD}

As the affective state of "Stress" is expected to cause a dilation of the pupil [24], the AIC output, i.e., the MPD signal, is further processed by a post-processing MATLAB function to isolate significant increases, which indicate the emergence of stress in the subject. This process is programmed in two steps:

(i) Zero out the negative portions of the MPD signal;

(ii) Apply a sliding window with a width of 900 samples throughout the non-negative MPD signal to calculate the median value within each window. 
The result of this procedure can be seen in Figure 5-4, where the middle panel shows the non-negative MPD signal after step (i) and the bottom panel is the outcome of this processing, termed the Processed MPD, or "PMPD" signal.

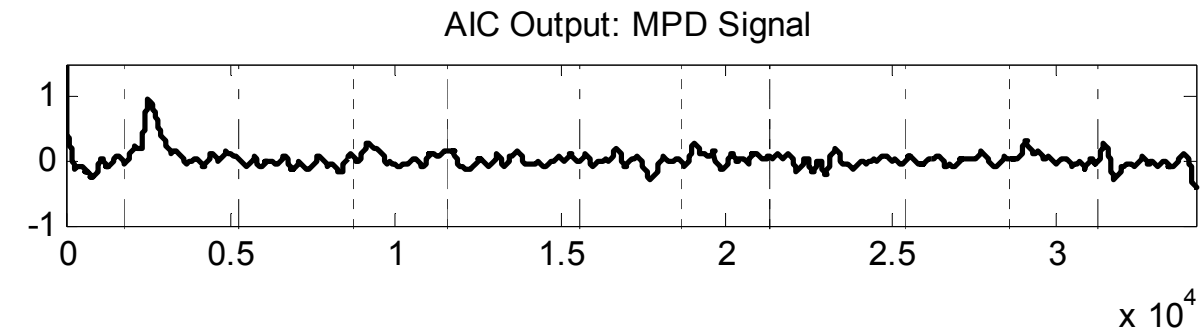

Non Negtive MPD Signal

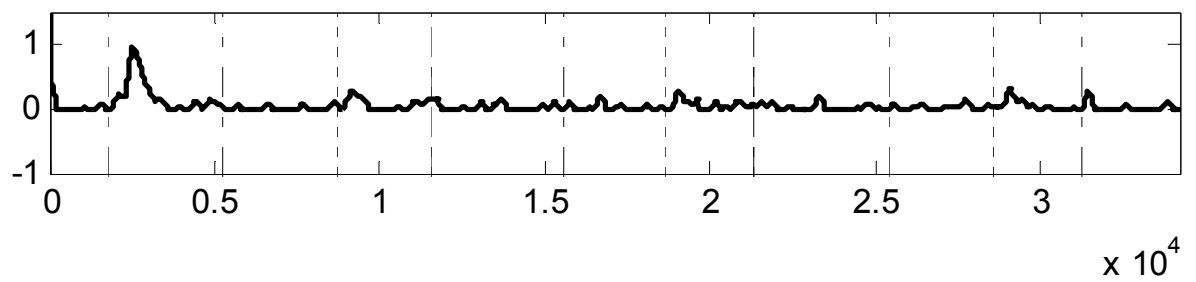

Sliding Window Mean Analysis of MPD

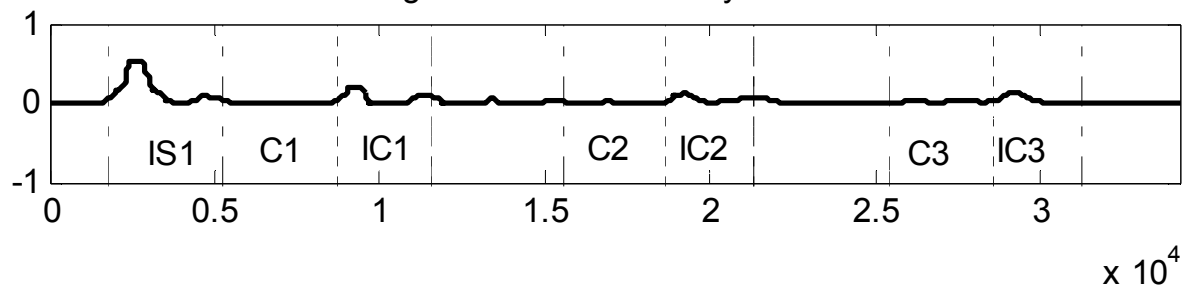

Figure 5-4: Post-processing of MPD signal

After this post-processing of the MPD signal, the mean value of the PMPD signal in each $\mathrm{C}$ and IC segments was obtained as an important PD feature for affective recognition. For example, the values of this "PMPDmean" feature recorded in the six Stroop segments for Subject 13 can be seen in Table 5-1

Table 5-1: PD feature from example (Subject 13)

\begin{tabular}{|c|c|c|c|c|c|c|}
\hline Segment & C1 & IC1 & C2 & IC2 & C3 & IC3 \\
\hline $\begin{array}{c}\text { PD Feature } \\
\text { ("PMPDmean") }\end{array}$ & 0.0034 & 0.0658 & 0.0048 & 0.0511 & 0.0168 & 0.0423 \\
\hline
\end{tabular}




\subsubsection{Discussion of PMPD \& PD Signals}
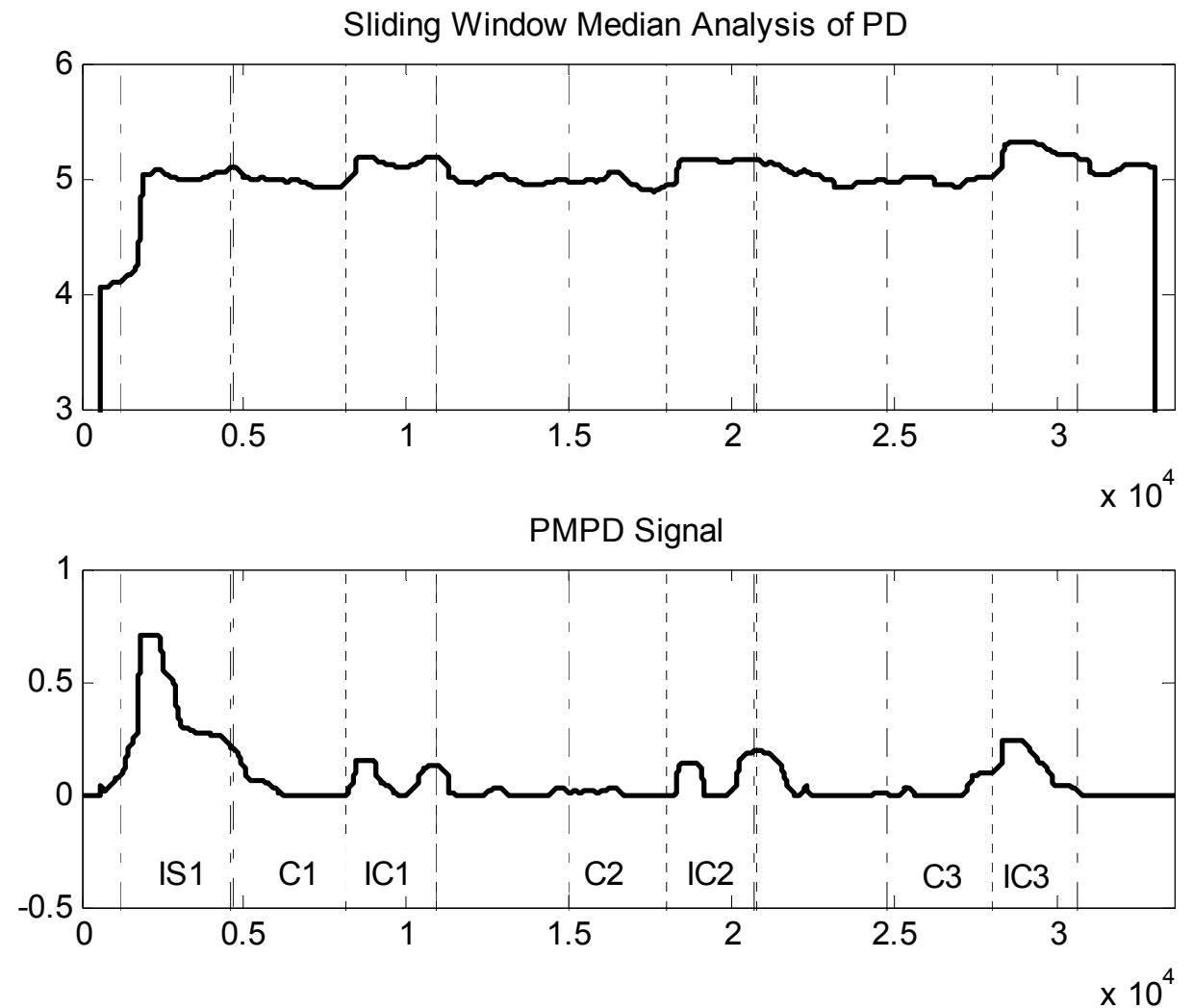

Figure 5-5: Comparison of PMPD with sliding window median analysis on PD

Figure 5-5 compares the result of applying the sliding median window to the PD signal directly (i.e., without processing it through the AIC) with the PMPD signal. From the plot of the PMPD signal (bottom pane of Figure 5-5), it is clear that the significant increases that have been isolated in the processed MPD signal correlate closely with the occurrence of IC segments, regardless of the presence of higher illumination passages during segments IC2 or $\mathrm{C} 3$.

The effect of this isolation is compared with the same post-processing operation on the PD signal, which has not been processed by AIC. Figure 5-5 shows that although the processed PD signal does respond to the "Stress" in the IC segments, it is not able to set apart the IC segments as clearly as the PMPD signal. 


\subsection{Signal Processing Results for the GSR Signal}

The recorded GSR signal was first down sampled from $360 \mathrm{~Hz}$ to $60 \mathrm{~Hz}$, and the portion of the recordings acquired during the performance of the Stroop Test was extracted for further processing.

To remove the recording noise, a 64 order low-pass FIR filter with cutoff frequency $2 \mathrm{~Hz}$ was applied to the GSR signal first. The filtered signal was shifted forward by 32 sampling intervals to maintain the time synchronization of the filtered signal. Figure 5-6 displays the comparison of the GSR signal before and after the filter, which shows the evident improvement in the signal quality. (The example here is the GSR signal in C2 from subject 13).
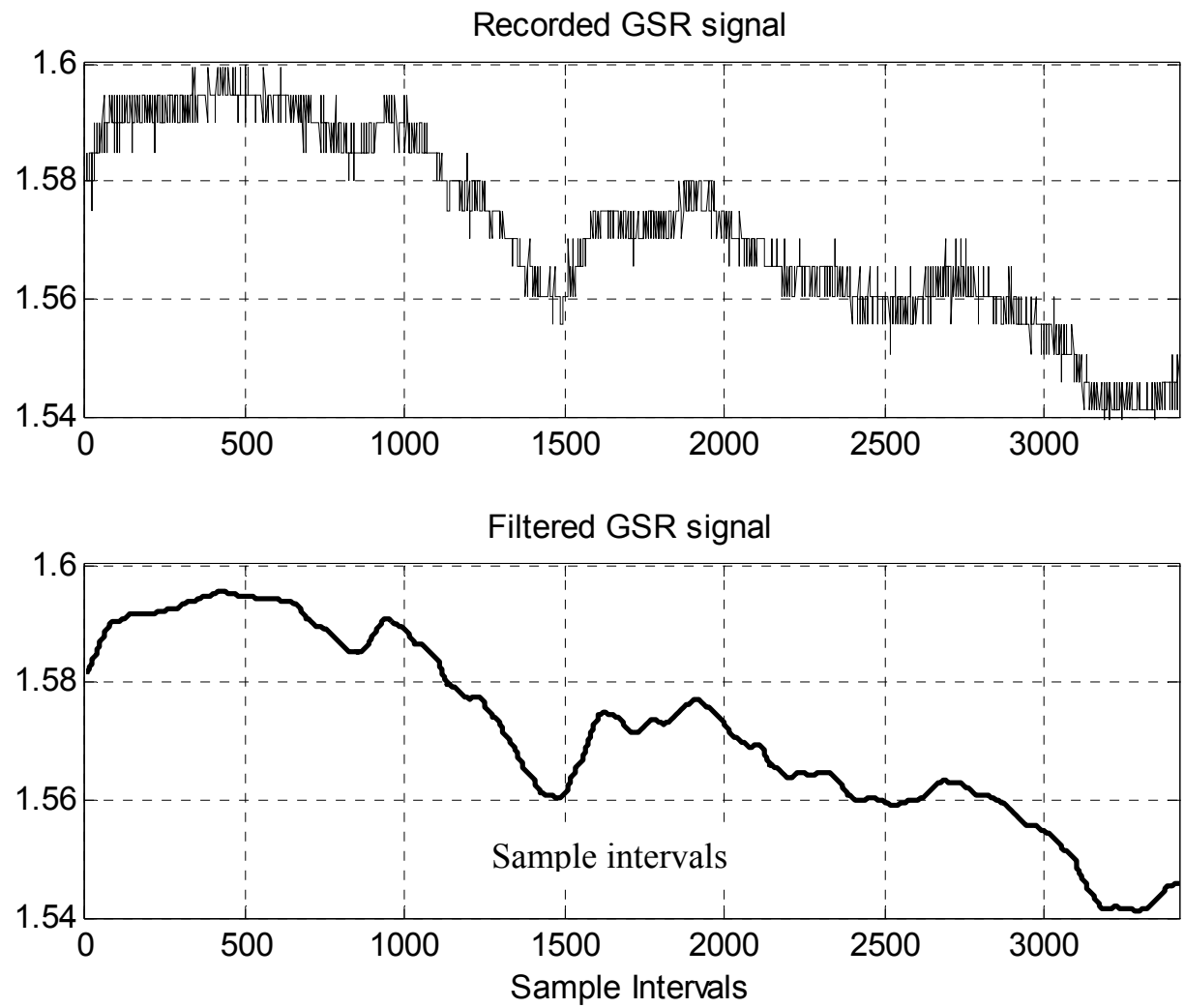

Figure 5-6: Comparison of GSR signal before and after the low pass FIR filter 
Here, the mean value of GSR in each C and IC segment was obtained as one of the important GSR features (In this example, GSRmean_C2_Subject13=1.5719).

Then, the signal was normalized using z-score normalization to achieve a signal with zero-mean and unit variance. The normalized example of GSR signal is shown in the top plot of Figure 5-7. Visually, four phasic responses (A, B, C, D) can be recognized from this GSR signal. These responses can be clearly distinguished from the background by using Ktonas' 7-point Lagrangian interpolation algorithm [54] to take the second time derivative of the signal. The bottom plot of Figure 5-7 shows the output of such Lagrangian interpolation approach with the sampling interval $h=100$, when it is applied to the segment shown in Figure 5-6.

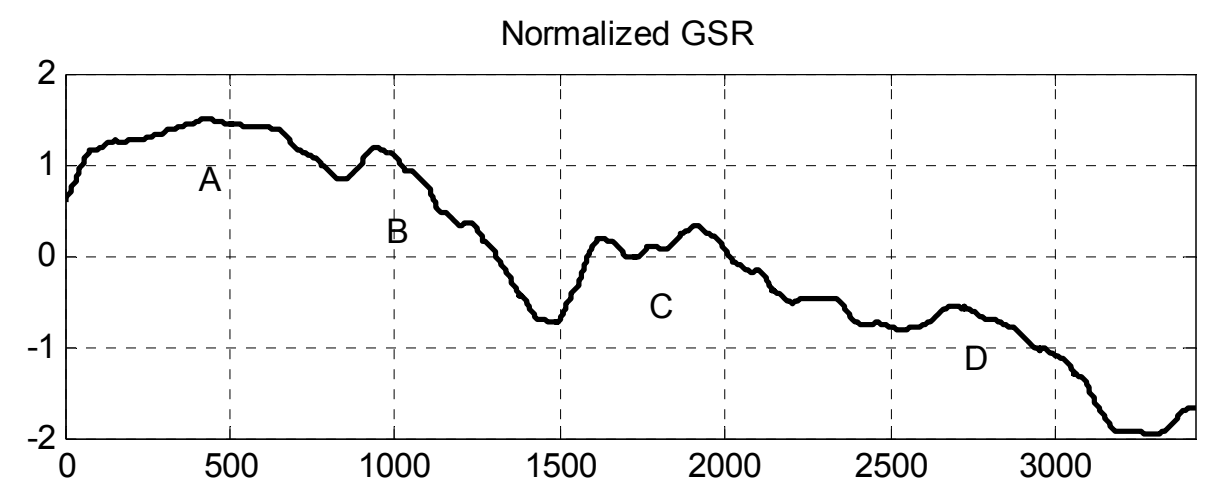

7-point Langrange Interpolation

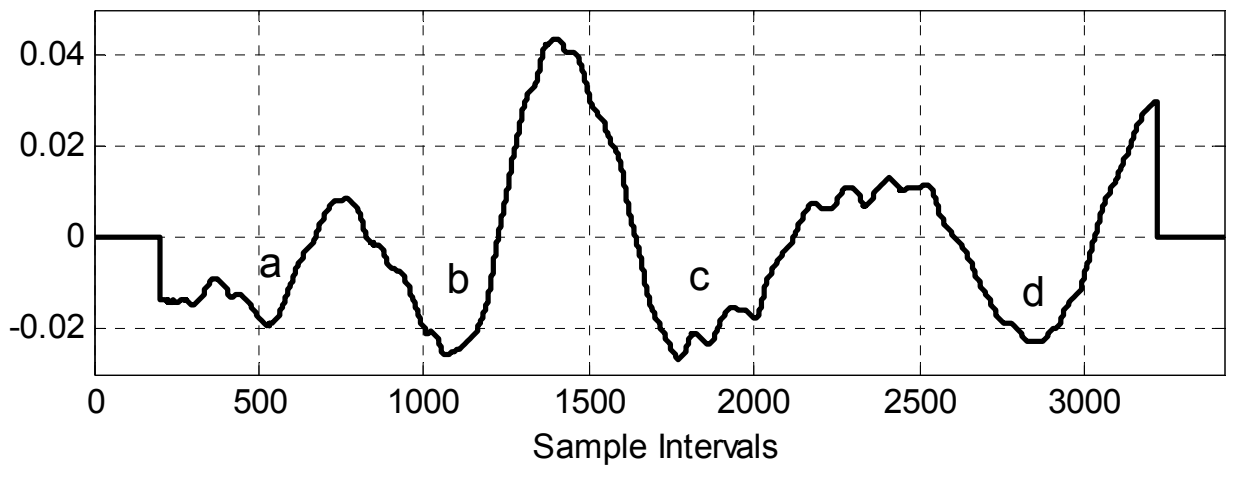

Figure 5-7: Z-score normalized GSR \& 7-point interpolator application 
In this second derivative sequence, the temporary increases due to GSR responses (such as $\mathrm{A}, \mathrm{B}, \mathrm{C}$ and $\mathrm{D}$, in the top panel of Figure 5-7) were represented by negative lobes, (such as a, b c and d, in the bottom panel of Figure 5-7), respectively. These lobes can be detected by thresholding (e.g., for this case, $t h=0.01$ ) and their time indices of occurrence can be referred back to the GSR signal (Figure 5-6) to isolate suspected GSR responses. False response indications from the second derivative sequence (e.g., "a" in Figure 5-7) were discounted when the amplitude was less than 0.05 volts in the original GSR signal. Therefore, in the case of this GSR example, only 3 responses can be acknowledged, which are "B", "C" and "D".

According to the previous description (Section 3.5 and 3.7), four other GSR features can be further derived from these isolated GSR response. In this example, the calculated value of all five features in the second Congruent segment (C2) of GSR signal from Subject 13 is summarized in the table below.

Table 5-2: Five GSR features from example (Subject 13 Segment C2)

\begin{tabular}{|c|c|c|c|c|}
\hline GSRmean. & GSRnum & GSRmeanAmp & GSRrisingTime & GSReng \\
\hline 1.5719 & 3 & 0.1316 & 2.4222 & 0.0164 \\
\hline
\end{tabular}

\subsection{Signal Processing Results from the BVP Signal}

The same as for PD, IL and GSR, the BVP signal of concern was edited to start 1800 samples ( 30 seconds) before the beginning of the Stroop Test itself, after it had been down sampled from $360 \mathrm{~Hz}$ to $60 \mathrm{~Hz}$. 
Then, a 64 order low pass FIR filter was applied to remove the recording noise, which is similar to the one used for the GSR signal, but with cutoff frequency of $6 \mathrm{~Hz}$. Figure 5-8 shows an example of the segment signal chosen from the $\mathrm{C} 2$ segment of the BVP recorded from subject 13. To keep the same waveform and preserve its time alignment, the filtered BVP signal was shifted forward 32 samples, which is plotted in Figure 5-8 as well. It should be noted that individual cardiac cycles are represented by the small cyclic variations of these signals.
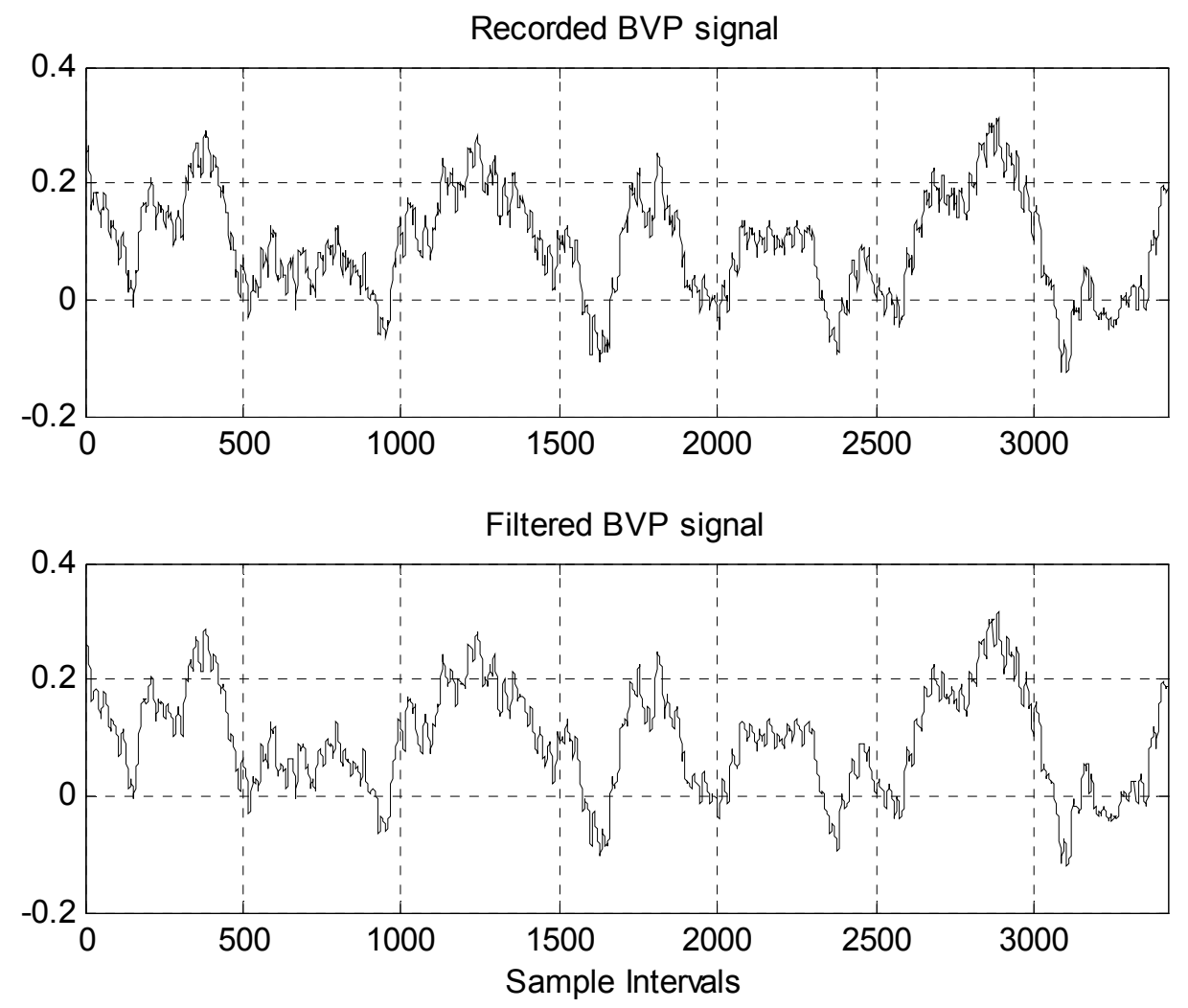

Figure 5-8: Comparison of BVP signal before and after the low pass FIR filter

It has been mentioned in Chapter 2 that the HRV information can be obtained from the high resolution BVP signal recorded, based on the inter-beat interval (IBI) data analysis. 
Figure 5-9 shows the first derivative of the example BVP signal. By thresholding ( 0.5 for this example), the accelerating phase of each BVP beat can be localized. The peak and valley of each BVP beat can be obtained by searching the local maximum and minimum in the BVP signal around the segment of highest first derivative. Each peak-to-peak interval was considered as the duration of one BVP beat, which was divided by the frequency $60 \mathrm{~Hz}$ and multiplied by 1000 to show the IBI in millisecond. The amplitude of each BVP beat was calculated as the difference between the peak and the valley. The average amplitude of all BVP beats in the segment under analysis was treated as the first physiological feature extracted from the BVP signal in each segment, since its decrease may indicate that the subject was undergoing mental stress.

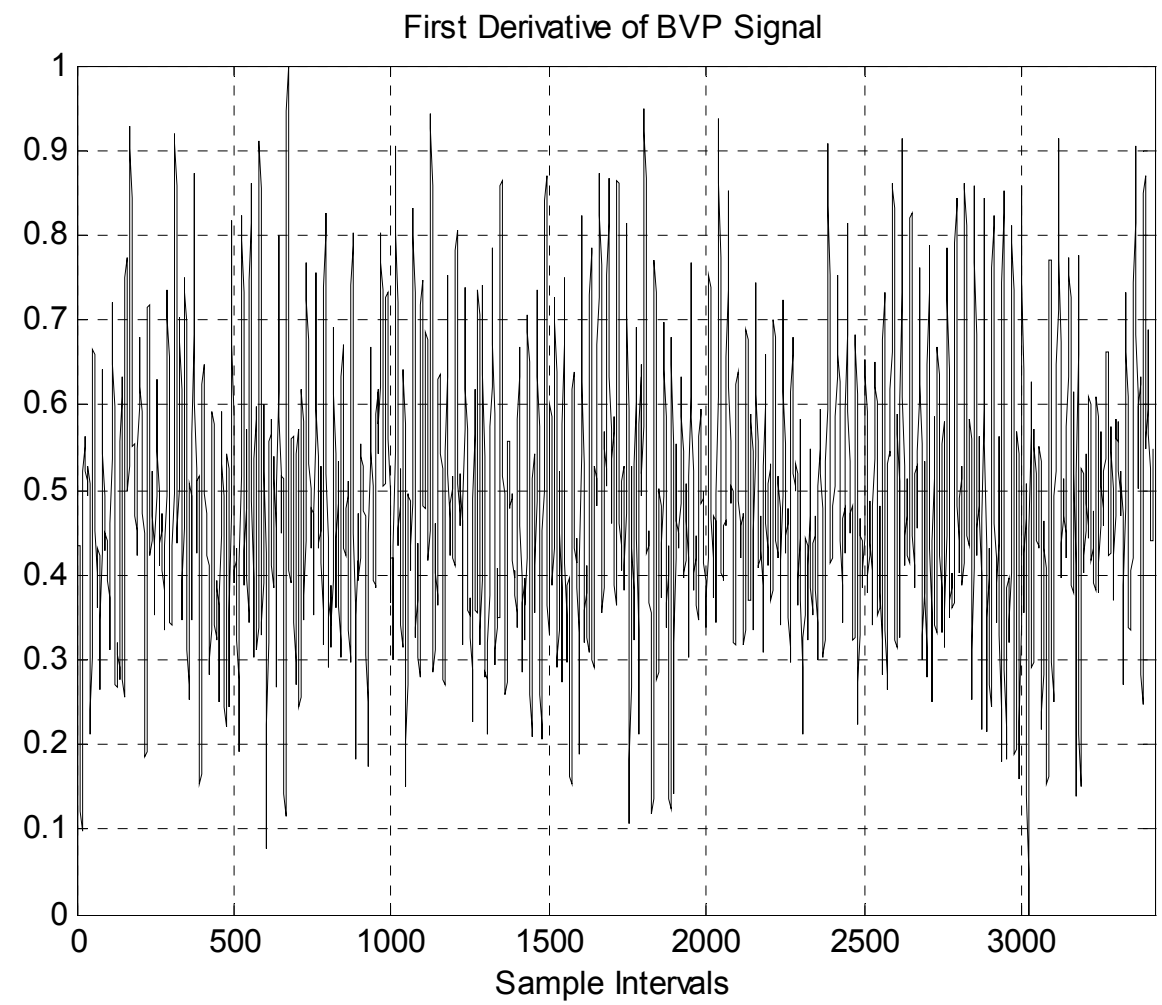

Figure 5-9: First derivative of BVP signal 
For the example BVP segment shown in Figures 5-8 and 5-9, the number of samples between two consecutive peaks of the signal was stemmed in the top panel of Figure 5-10 and the heart beat duration in milliseconds, or the inter-beat interval (IBI) sequence was plotted in the bottom. The mean value and standard deviation of this IBI sequence were extracted as the second and third features to represent the BVP characteristics of each segment.
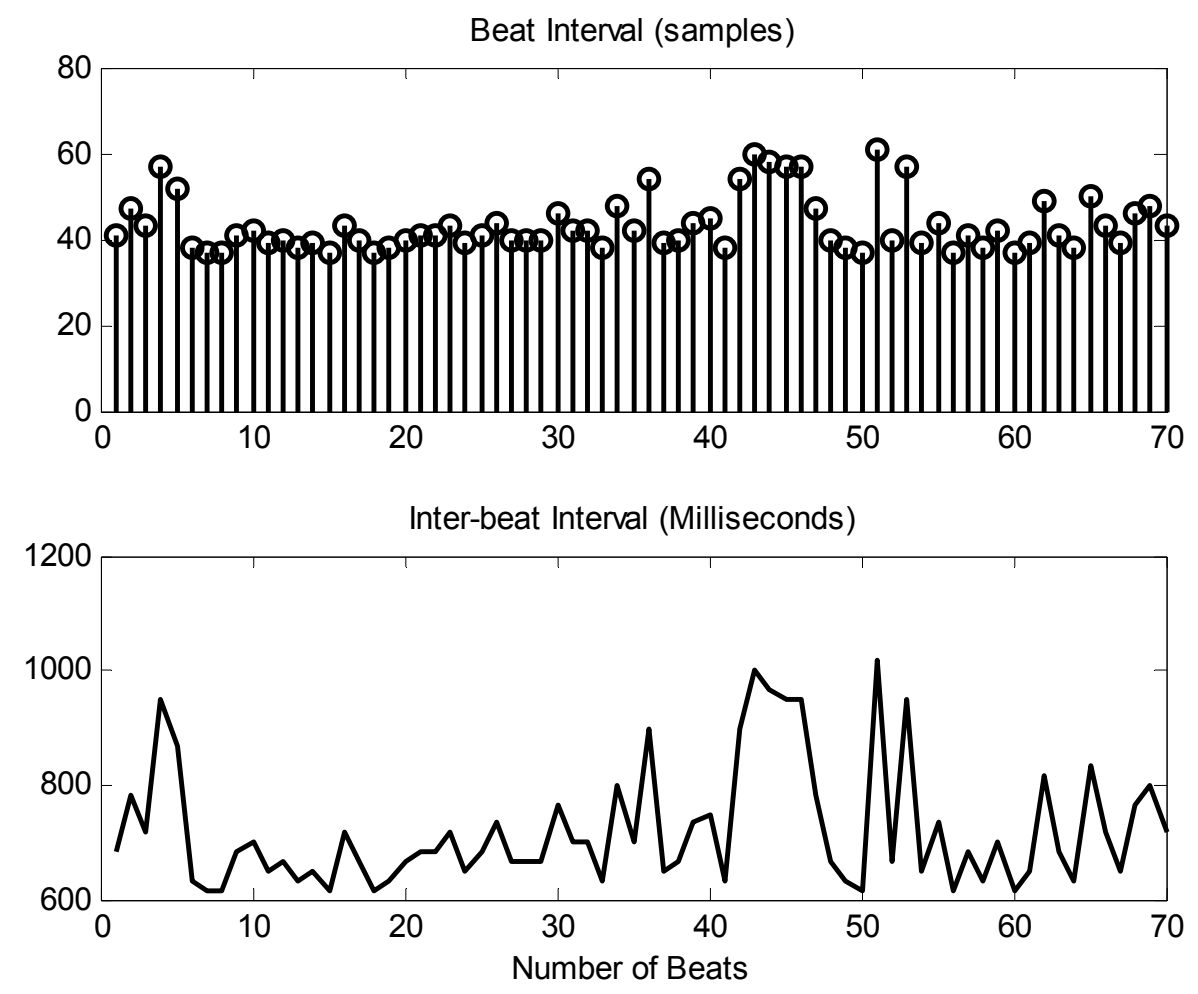

Figure 5-10: Heart beat intervals in samples and milliseconds

To obtain the last BVP feature, the IBI signal was also processed by power spectrum analysis to extract frequency domain information. As described in Section 3.6, the accumulation of the power spectrum values within each spectral region (VLF, LF and HF) was calculated. The feature LF/HF ratio was expected display a significant increase in the stress (IC) segments. In case of this BVP example, the four features in the second 
Congruent segment (C2) of the Stroop Test from Subject 13 are summarized in the following table.

Table 5-3: Four BVP features from example (Subject 13 Segment C2)

\begin{tabular}{|c|c|c|c|}
\hline BVPmeanAmp & BVPIBImean & BVPIBIstd & BVPI2h \\
\hline 0.0067 & 0.7221 & 0.1063 & 0.8169 \\
\hline
\end{tabular}

\subsection{Physiological Features Extracted}

After the signal processing of PD signal (together with IL) using the AIC system with the HITV adaptive algorithm, the PMPD signal is obtained. The data collected from Subject 13 is shown in Figure 5-11, where the two top signals (GSR and BVP, which was recorded simultaneously) have been shifted up to make the display clear.

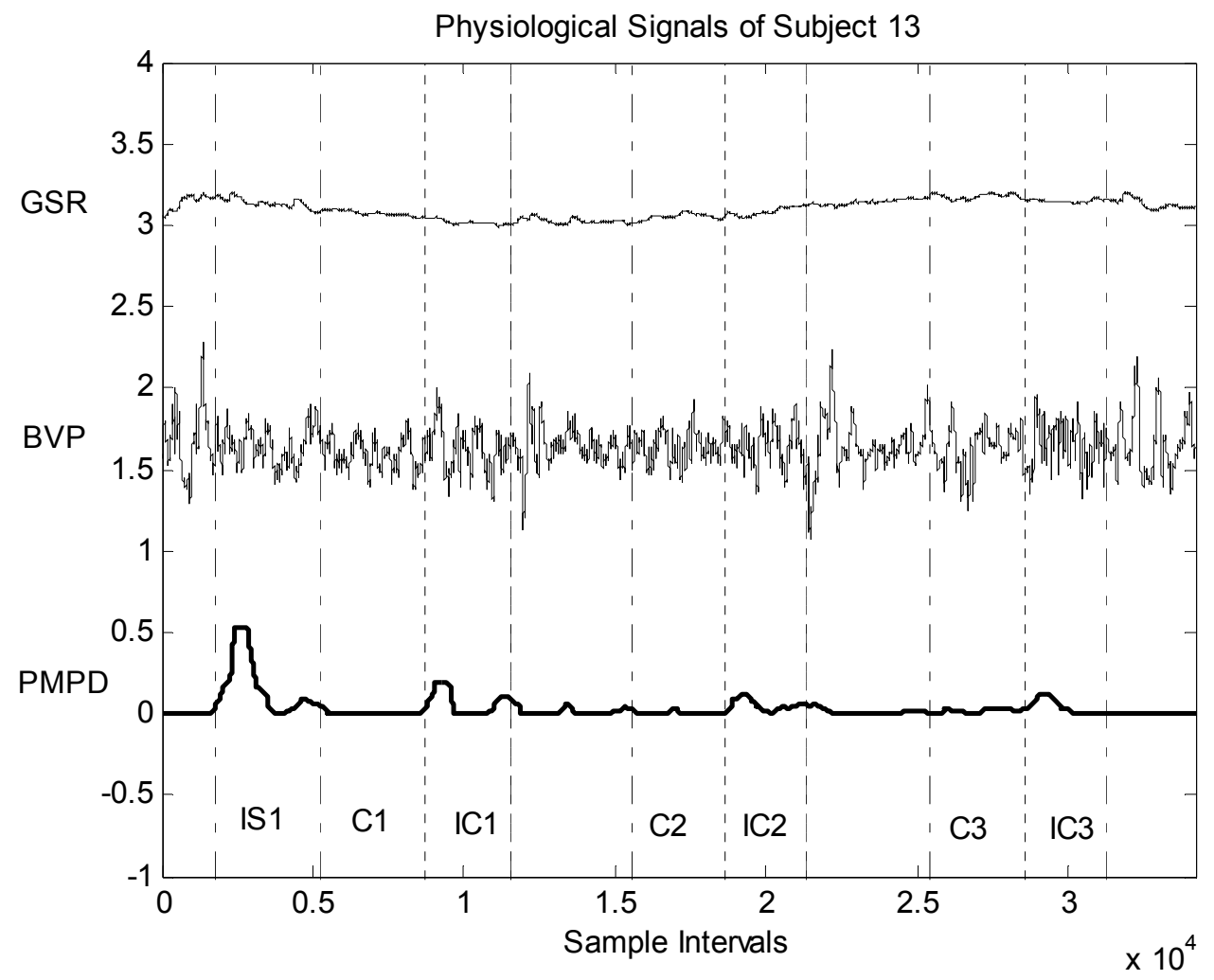

Figure 5-11: Signal processing results for Subject 13. 
From the figure, it is clear that the most significant increases of the bottom trace, after the initial adaptation that precedes segment IS1, occur during the Incongruent segments (IC1, IC2 and IC3). And, compared with other two signals, the PMPD gives the best visually differentiation of IC segments from the whole test, which encourages the using of the PD feature, "PMPDmean" for stress classification.

As an example of the feature values obtained, the 10 features extracted from PMPD, GSR, and BVP throughout the experiment are illustrated in the table below. This table also exemplifies how the "PMPDmean" feature takes on clearly different values in $\mathrm{C}$ segments and in IC segments.

Table 5-4: Values of all 10 features from Subject 13

\begin{tabular}{|c|c|c|c|c|c|c|c|}
\hline \multirow{2}{*}{ Signals } & \multirow{2}{*}{ Features } & \multicolumn{6}{|c|}{ Stroop Test } \\
\hline & & C1 & IC1 & $\mathrm{C} 2$ & IC2 & C3 & IC3 \\
\hline PD & PMPDmean & 0.0034 & 0.0658 & 0.0048 & 0.0511 & 0.0168 & 0.0423 \\
\hline \multirow{5}{*}{ GSR } & GSRmean. & 1.5718 & 1.5182 & 1.5499 & 1.5814 & 1.67461 & 1.6479 \\
\hline & GSRnum & 2 & 3 & 3 & 5 & 4 & 4 \\
\hline & GSRmeanAmp & 0.1736 & 0.2174 & 0.7316 & 0.441 & 0.8085 & 0.8291 \\
\hline & GSRrisingTime & 3.55 & 2.7944 & 4.9833 & 6.0167 & 5.0833 & 4.9875 \\
\hline & GSReng & 0.0163 & 0.1065 & 0.2642 & 0.626 & 1.2732 & 0.2406 \\
\hline \multirow{4}{*}{ BVP } & BVPmeanAmp & 0.0093 & 0.0173 & 0.0175 & 0.0103 & 0.0037 & 0.0287 \\
\hline & BVP12h & 0.4918 & 0.4497 & 0.276 & 0.6419 & 0.5579 & 0.8531 \\
\hline & BVPIBImean & 0.7451 & 0.7787 & 0.8052 & 0.7642 & 1.127 & 0.9659 \\
\hline & BVPIBIstd & 0.1194 & 0.1537 & 0.1726 & 0.1626 & 0.5728 & 0.3807 \\
\hline
\end{tabular}




\subsection{Data Normalization}

Thirty subjects participated in the experimental protocol used for this study, which yielded a total of 6 (segments) $\times 30$ (subjects $)=180$ segment values for each of the 10 features introduced in the previous sections. For each feature, half of these segments correspond to "stressed" states induced by IC Stroop stimulation, and the rest are known to be associated with "relaxed" (C Stroop) intervals.

To minimize the impact of the characteristics of individual responses on the SVM training, these feature data were normalized (according to the process outlined in Section 3.7), before its application to the machine learning classification. As an example of the normalization results, Figure 5-12 illustrates the separation between the values of "PMPDmean" feature for Congruent and Incongruent segments, after the normalization.

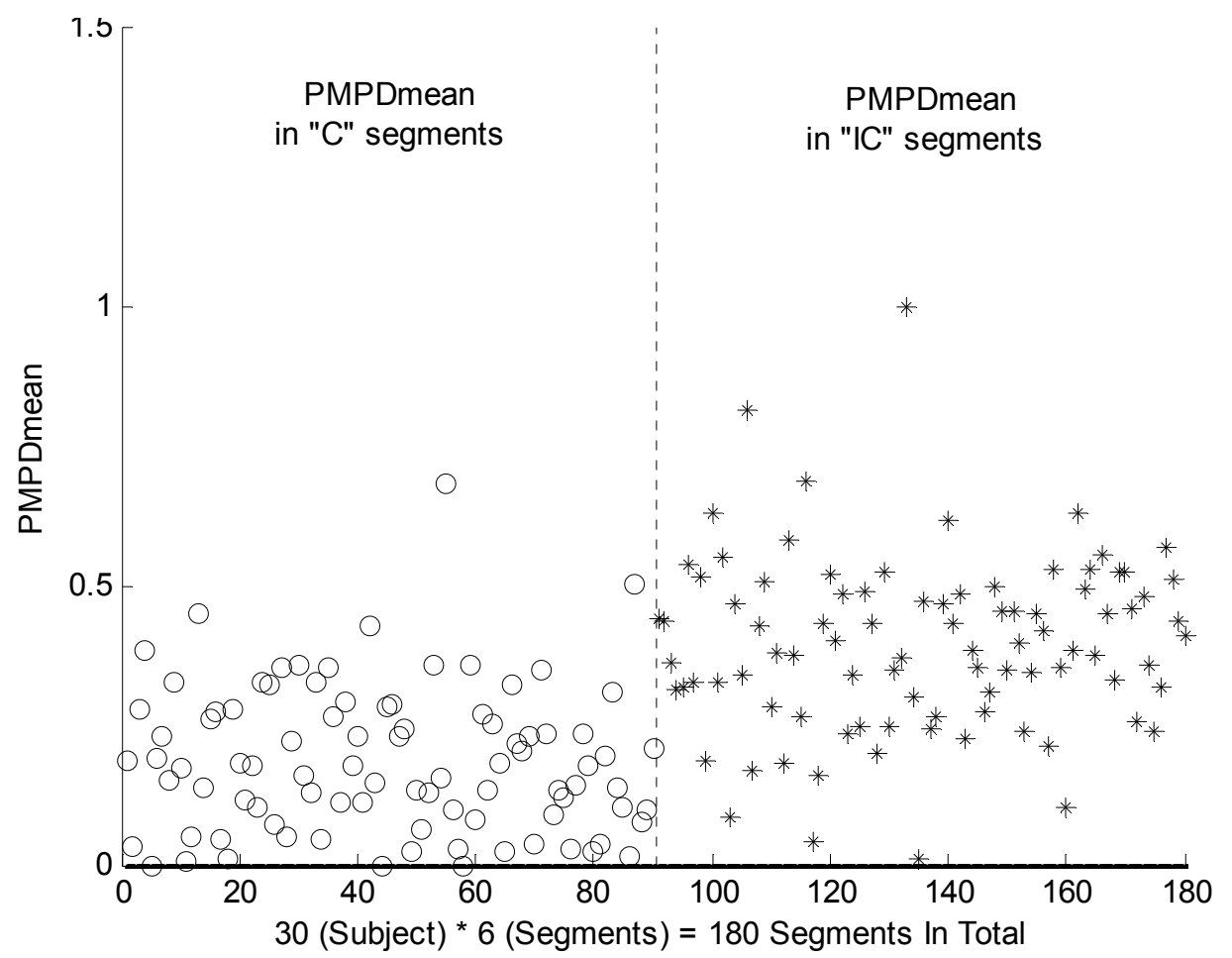

Figure 5-12: The distribution of "PMPDmean" feature values for both types of segments 
In this figure the values of the normalized "PMDPmean" feature for all the 90 Congruent (i.e., "relaxed") segments are plotted first, indicated with "o" markers, followed by the 90 "PMDPmean" values obtained from Incongruent (i.e., "stressed") segments, indicated with " " " markers. This plot confirms that, indeed, the values of the

"PMDPmean" feature corresponding to the two classes under study are clustered around different values.

\section{6 "Stress" Classification with SVM}

After all features are extracted from the physiological signals monitored, the feature sets are normalized and further processed by a pattern classification system, to attempt differentiation of the "stress" state (IC Stroop segments) from the normal state (C Stroop segments) of a person working on a computer task. Given the context of this project, support vector machines were chosen as the machine learning classification systems to use.

Data classification by means of SVM is a two-step process. In the first step, a model is built to describe a predetermined set of data classes based on the training data set. In the second step the model built through the training phase is used for classification. The classification accuracy of the model (or classifier) is estimated by the percentage of test set samples that are correctly classified by the model.

In this study, a particularly interest was placed on determining the stress classification capability achieved by the pupil diameter monitoring and processing, since 
its use for affective assessment had not been fully studied, in contrast with other physiological signals, such as GSR and BVP.

To assess the overall usefulness of the pupil diameter signal, the classification phase of this project was repeated under 3 different conditions:

(i) Only using the feature extracted PD (only 1 feature, "PMPDmean" was used for classification);

(ii) Using all features extracted from the monitored PD, GSR and BVP signals (all 10 features were used for classification);

(iii) Excluding the feature extracted from PD signal; (1 feature "PMPDmean" was excluded, 9 features from GSR and BVP were used for classification).

It should be noted that the prediction performance was evaluated using the 20 -fold cross validation strategy, in each case, to gauge the system classification accuracy in a realistic fashion.

The experimental data from the 180 segments collected from the 30 volunteers were processed through the protocol described, to train and verify the performance of the SVM learning classification systems. The accuracy rates from the experiments performed to evaluate the proposed affect recognition system by SVM using the "SPIDER" software package for MATLAB are shown in Table 5-5.

The performance levels observed for the three phases of classification, measured by their total classification accuracy, will be discussed in the following section. 
Table 5-5: Results of stress classification by support vector machine

\begin{tabular}{|l|c|c|c|c|}
\hline \multirow{2}{*}{\begin{tabular}{c}
\multirow{2}{*}{$\begin{array}{c}\text { Phase of } \\
\text { Classification }\end{array}$} \\
\cline { 2 - 5 }
\end{tabular}} & Linear & Polynomial & RBF & Gaussian \\
\cline { 2 - 5 } & & $d=3.0$ & $\gamma=1.0$ & $\gamma=1.0$ \\
\hline P1: 1 feature from PD & $77.78 \%$ & $77.78 \%$ & $77.78 \%$ & $77.78 \%$ \\
\hline $\begin{array}{l}\text { P2: 10 features from } \\
\text { PD, GSR and BVP }\end{array}$ & $76.11 \%$ & $76.67 \%$ & $73.33 \%$ & $37.78 \%$ \\
\hline $\begin{array}{l}\text { P3: 9 features from } \\
\text { GSR and BVP (PD } \\
\text { Removed) }\end{array}$ & $50.00 \%$ & $54.44 \%$ & $48.89 \%$ & $37.78 \%$ \\
\hline
\end{tabular}

\subsection{Discussion of "Stress" Classification with SVM}

The outcomes of the SVM classification show that, under controlled conditions, the simultaneous monitoring and concurrent processing of PD signal, yields acceptable levels (up to 77.78\%) of differentiation between "non-stressed" and "stressed" user states, as evoked by Congruent and Incongruent Stroop stimulation, respectively. Regardless of which kernel was utilized, the SVM classifier is able to deal with the complex pattern distribution, achieving encouraging results for affective recognition.

The interaction of the information provided by the PD signal with other two physiological signals is also interesting. When the features from GSR and BVP are added to "PMPDmean" into the affective classification process, the observed system accuracy decreases slightly (up to $76.67 \%$ ). This seems to hint that the discrimination performance of the GSR and BVP features is not as good as that of the PMPD feature. 
In the third phase of the stress classification, it is also observed that with the PD signal excluded, the recognition rate dramatically dropped to $54.44 \%$ (for the best classifier), which is significantly lower than the accuracy rate in the previous two phases (both of which include "PMPDmean"). This result seems to indicate that the pupil diameter signal may be one of the most important physiological signals to involve in the development of an automated affective recognition system.

In order to obtain more information about the affective sensing performance of the PD signal, the PD feature ("PMPDmean") was used in additional SVM classification experiment as described in the following section.

\subsection{Further Discussion of Affective Sensing through a PD Feature with SVM}

As mentioned in Chapter 4, the Stroop Test is mainly composed of $3 \mathrm{C}$ and 3 IC segments. Additionally, illumination increases were introduced in two of these 6 segments. Therefore, as indicated in Figure 4-6, there are four kinds of experimental situations throughout the test. Accordingly, the PD feature, "PMPDmean" in these 6 Stroop segments of each subject can be divided into four groups according to the different factors that cause the PD variation (Table 5-6 ).

In this research, there are 30 subjects participated in the experimental protocol, which yielded a total of 30 segment values of the PD feature for G1 and G3. These 30 subjects also generate 60 segment values for G0 and G2. For each group, the SVM with polynominal kernel $(d=2.0)$ was applied to evaluate the affective sensing performance for each case of the PD variation, and the classification results are listed in the Table 5-7. 
Table 5-6: Four groups of PD feature (“PMPDmean”) for each subject

\begin{tabular}{|c|c|c|c|}
\hline \multirow{2}{*}{ Group } & \multicolumn{2}{|c|}{ PD changes caused by } & \multirow{2}{*}{ Segment(s) in Stroop Test } \\
\cline { 2 - 3 } & PAR & PLR & C1 \& C2 \\
\hline G0 & No & No & C3 \\
\hline G1 & No & Yes & IC1 \& IC3 \\
\hline G2 & Yes & No & IC2 \\
\hline G3 & Yes & Yes & \multicolumn{2}{c}{} \\
\hline
\end{tabular}

Table 5-7: SVM results for four groups of the "PMPDmean" feature

\begin{tabular}{|c|c|c|c|c|c|}
\hline \multirow{2}{*}{$\begin{array}{c}\text { Group (number } \\
\text { of values) }\end{array}$} & \multicolumn{3}{|c|}{ Classification results (number of results) } & \multirow{2}{*}{$\begin{array}{c}\text { Accuracy } \\
\text { Rate }\end{array}$} \\
\cline { 2 - 5 } & TN & TP & FN & FP & \\
\hline G0 (60) & 51 & 0 & 0 & 9 & $85 \%$ \\
\hline G1 (30) & 22 & 0 & 0 & 8 & $73.33 \%$ \\
\hline G2 (60) & 0 & 45 & 15 & 0 & $75 \%$ \\
\hline G3 (30) & 0 & 23 & 7 & 0 & $76.67 \%$ \\
\hline
\end{tabular}

The SVM results of G2 and G3 shows that the AIC successfully minimized the influence of the PLR in the measured PD signal. Although the classifier can achieve an accuracy rate of $75 \%$ in G2, where the PD signal variations are caused by the PAR only, it reaches $76.67 \%$ for G3, in which the PLR were purposely triggered in addition to the PAR, by increasing the light intensity above the subject's eyes.

However, there are multiple false negative (FN) outcomes in G2 and G3. It was speculated that maybe the "PMPDmean" feature was not sensitive enough. To explore 
this idea further, the GSR feature, "GSRmean", was added to the "PMPDmean" feature in a new SVM study. The test and training results are shown in Table 5-8.

Table 5-8: SVM results for four groups of the "PMPDmean" and "GSRmean" features

\begin{tabular}{|c|c|c|c|c|c|}
\hline \multirow{2}{*}{$\begin{array}{c}\text { Group (number } \\
\text { of values) }\end{array}$} & \multicolumn{3}{|c|}{ Classification results (number of results) } & Accuracy \\
\cline { 2 - 6 } & TN & TP & FN & FP & \\
\hline G0 (60) & 51 & 0 & 0 & 9 & $85 \%$ \\
\hline G1 (30) & 22 & 0 & 0 & 8 & $73.33 \%$ \\
\hline G2 (60) & 0 & 46 & 14 & 0 & $76.67 \%$ \\
\hline G3 (30) & 0 & 23 & 7 & 0 & $76.67 \%$ \\
\hline
\end{tabular}

The result of this SVM classification shows that the added GSR feature does not seem to improve the affective recognition performance of the PD feature. The accuracy rate of the SVM classifier is close to its performance in Table 5-7. Moreover, the number of False Negative (FN) classification results in G2 \& G3 is close to that in the previous SVM study as well, which hints that the instances of FN results maybe caused by a low response of the subjects, in those cases, to the stimuli in the Stroop Test.

In order to compare single-feature SVM classifier performance based on the PD feature and the GSR feature, the "GSRmean" was used separately in a SVM classification as well. 
Table 5-9: SVM results for four groups of the "GSRmean" feature alone

\begin{tabular}{|c|c|c|c|c|c|}
\hline \multirow{2}{*}{$\begin{array}{c}\text { Group (number } \\
\text { of values) }\end{array}$} & \multicolumn{4}{|c|}{ Classification results (number of results) } & $\begin{array}{c}\text { Accuracy } \\
\text { Rate }\end{array}$ \\
\cline { 2 - 6 } & TN & TP & FN & FP & 40 \\
\hline G0 (60) & 20 & 0 & 0 & $33.33 \%$ \\
\hline G1 (30) & 8 & 0 & 0 & 22 & $26.67 \%$ \\
\hline G2 (60) & 0 & 51 & 9 & 0 & $85 \%$ \\
\hline G3 (30) & 0 & 27 & 3 & 0 & $90 \%$ \\
\hline
\end{tabular}

The SVM results in Table 5-9 show that the SVM classification of the "GSRmean" feature provides high sensitivity. It reaches a high accuracy rate of $85 \%$ in G2 and $90 \%$ in G3, where the PAR is triggered by the IC Stroop segments. However, it is not selective enough, since its classification rate in G0 and G1 is low (33.33\% for G0 and $26.67 \%$ for G1).

To supplement the analysis of the classification performance achieved in the previous SVM studies, the classification potential from each single feature derived from the PD, GSR and BVP signals is investigated separately in Section 5.9 through the ROC analysis (one feature at a time).

\subsection{Discussion of Single Feature Discriminating Capability with ROC}

To assess the comparative discriminating capability of each of the features listed in Table 3-2, their individual ROC curves were plotted and an estimation of the area under each of them (AUROC) was obtained based on the 180 segment values from each normalized feature calculated in this study. For each feature, half of these segments 
correspond to "stressed" states induced by IC Stroop stimulation (ideal classifier output = 1), the other half are known to be associated with "relaxed" (C Stroop) intervals (ideal classifier output $=-1)$.

In the ROC analysis, each point of the curve is determined by comparing the value of the single detection signal to a specific threshold and determining which portion of the "positive classifier outputs" match the ideal output (1) and which portion of the "negative classifier outputs" are in disagreement to the ideal output (-1). These "portions", expressed as fractional numbers, yield the coordinates of the ROC point (false positive rate, true positive rate) for the threshold value tested. The process was carried out using the ROC MATLAB ${ }^{\circledR}$ scripts provided by Dr. Gavin C. Cawley. These scripts not only sweep the complete range of normalized threshold values, $[0,1]$, and draw the ROC, but additionally estimate a "convex hull" that fits the actual ROC points calculated. The convex hull is shown with dashed lines in the resulting plots. Additionally, the AUROC is calculated for each curve.

The resulting AUROC values (Table 5-10) reveal that the "PMPDmean" feature has the highest discriminating power, followed distantly by the "GSRmean" feature. The ROC curves for these 10 features are presented next, in Figure 5-13 to Figure 5-22. 
Table 5-10: AUROC results for all 10 features

\begin{tabular}{|c|c|c|}
\hline Signal & Detection Feature & AUROC \\
\hline PD ( 1 feature) & PMPDmean & 0.8546 \\
\hline \multirow{5}{*}{ GSR (5 features) } & GSRmean. & 0.6756 \\
\hline & GSRnum & 0.4196 \\
\hline & GSRmeanAmp & 0.4285 \\
\hline & GSRrisingTime & 0.4915 \\
\hline & GSReng & 0.4704 \\
\hline \multirow{4}{*}{ BVP (4 features) } & BVPmeanAmp & 0.4599 \\
\hline & BVP12h & 0.5432 \\
\hline & BVPIBImean & 0.3991 \\
\hline & BVPIBIstd & 0.5031 \\
\hline
\end{tabular}

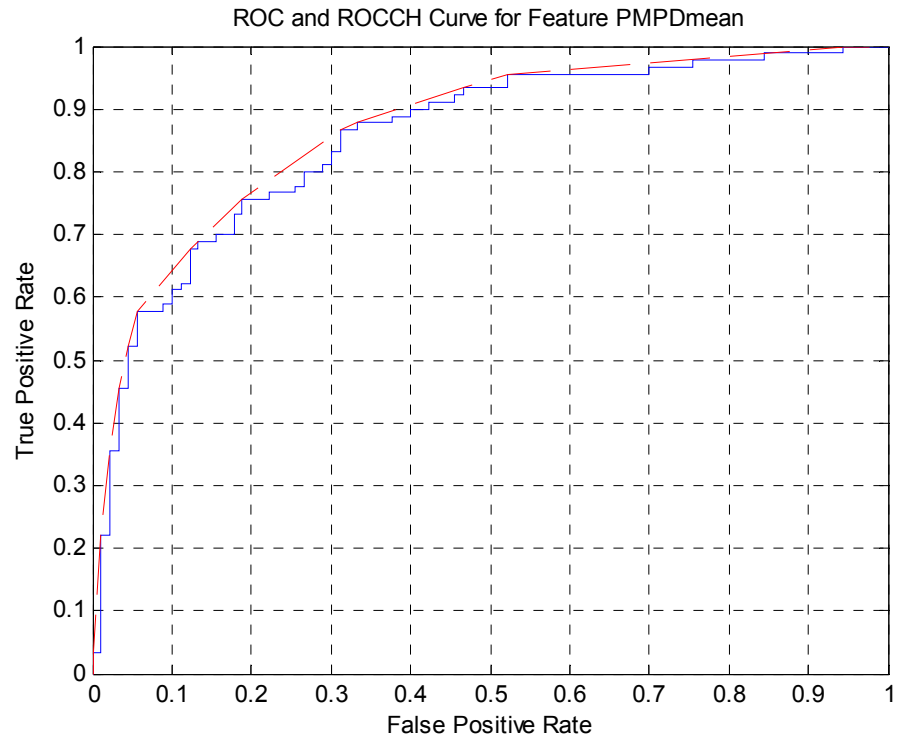

Figure 5-13: "PMPDmean" ROC curve $($ AUROC = 0.8546) 


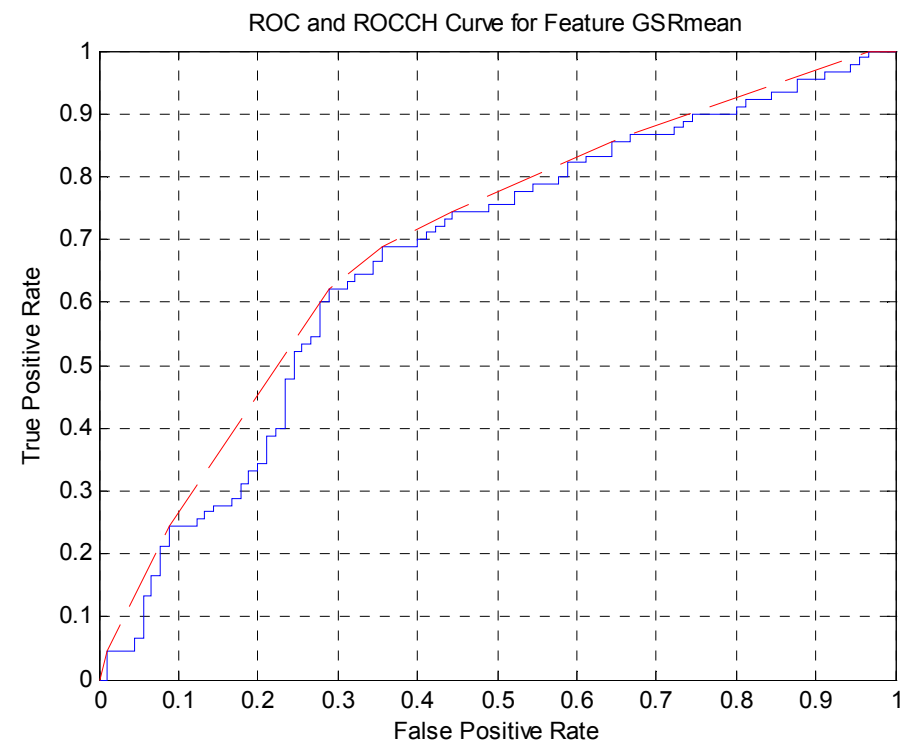

Figure 5-14: “GSRmean” ROC curve $($ AUROC = 0.6756)

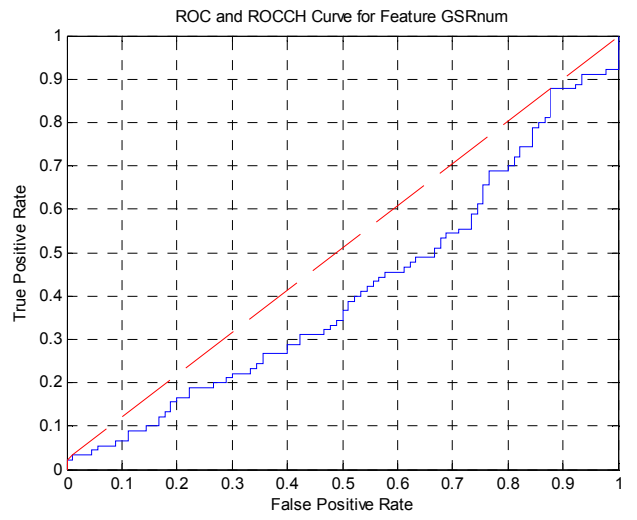

Figure 5-15: "GSRnum" ROC curve $(\mathrm{AUROC}=0.4196)$

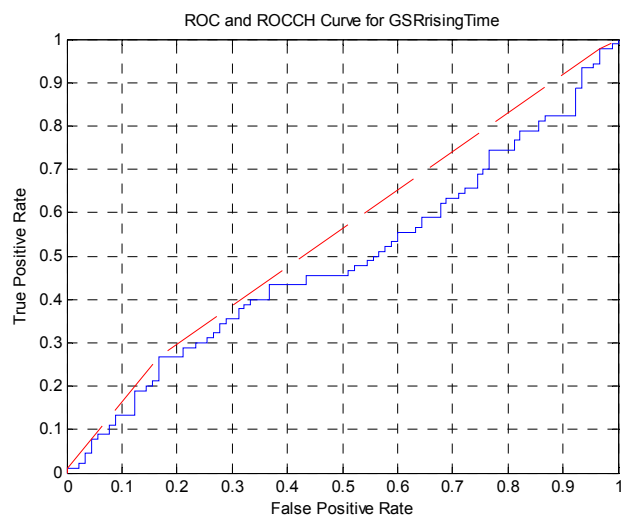

Figure 5-17: "GSRrisingTime" ROC curve $($ AUROC $=0.4915)$

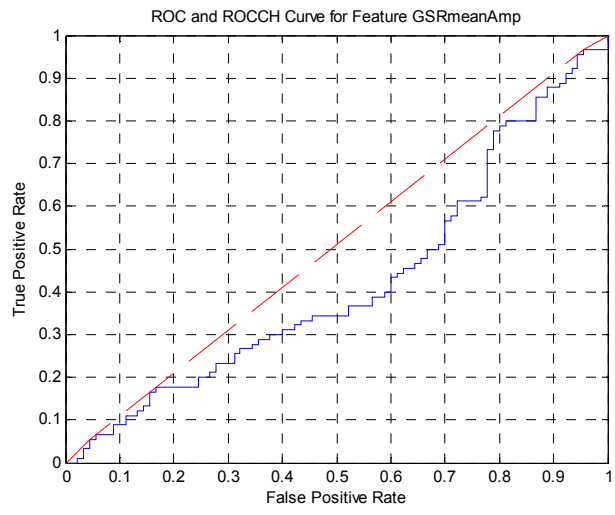

Figure 5-16: "GSRmeanAmp” ROC curve $($ AUROC $=0.4285)$

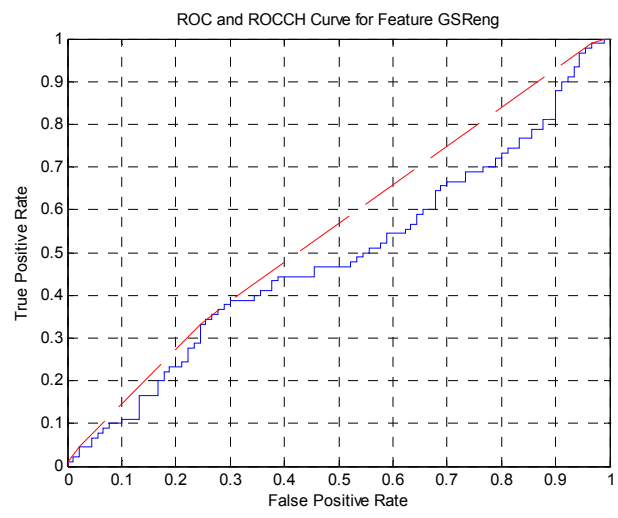

Figure 5-18: "GSReng” ROC curve $(\mathrm{AUROC}=0.4704)$ 


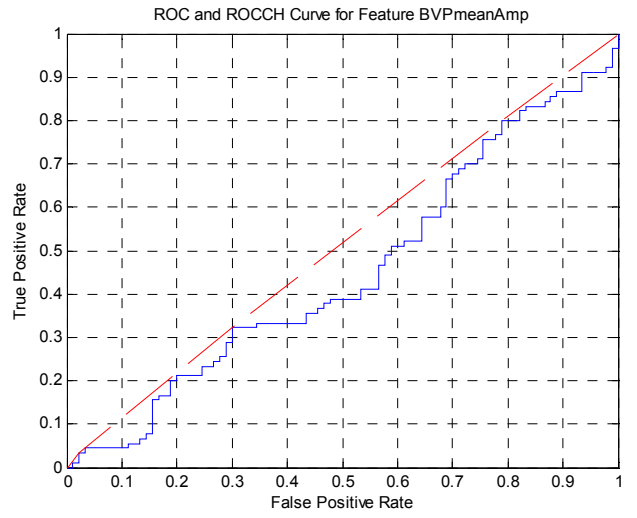

Figure 5-19: "BVPmeanAmp" ROC curve $($ AUROC $=0.4599)$

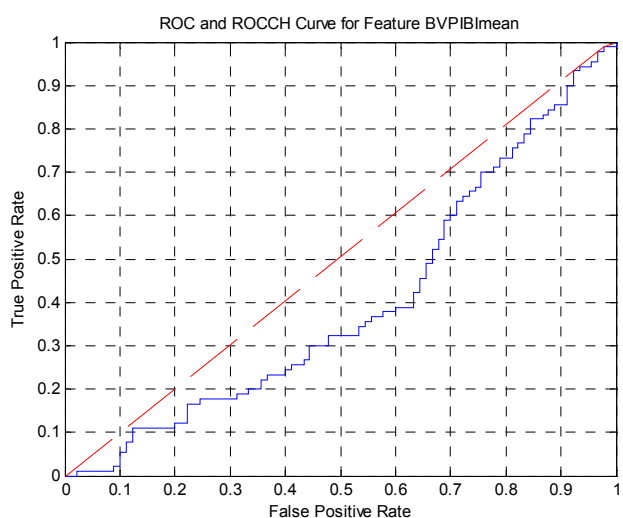

Figure 5-21: "BVPIBImean" ROC curve $(\mathrm{AUROC}=0.3991)$

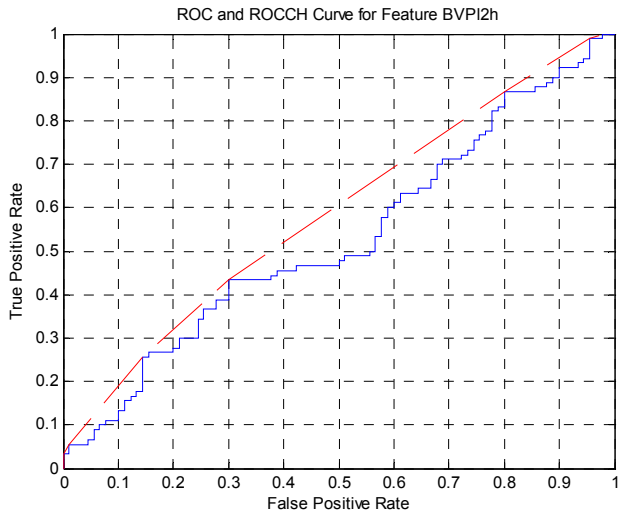

Figure 5-20: "BVP12h" ROC curve $(\mathrm{AUROC}=0.5432)$

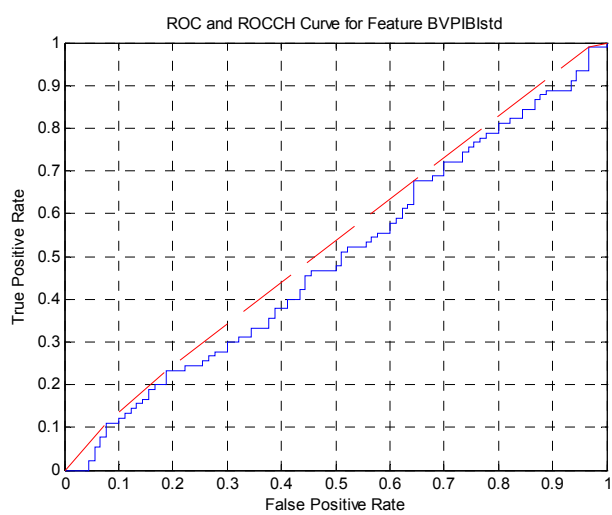

Figure 5-22: "BVPIBIstd" ROC curve $(\mathrm{AUROC}=0.5031)$

From the ROC analysis results for all the 10 features, it is noticeable that the ROC curve for "PMPDmean" shows a convexity, which brings its area closer to 1 (AUROC_PMPDmean $=0.8546)$. In contrast, only some of the ROC curves for the other features show a slight upwards deflection in their trajectories from $(0,0)$ to $(1,1)$. 


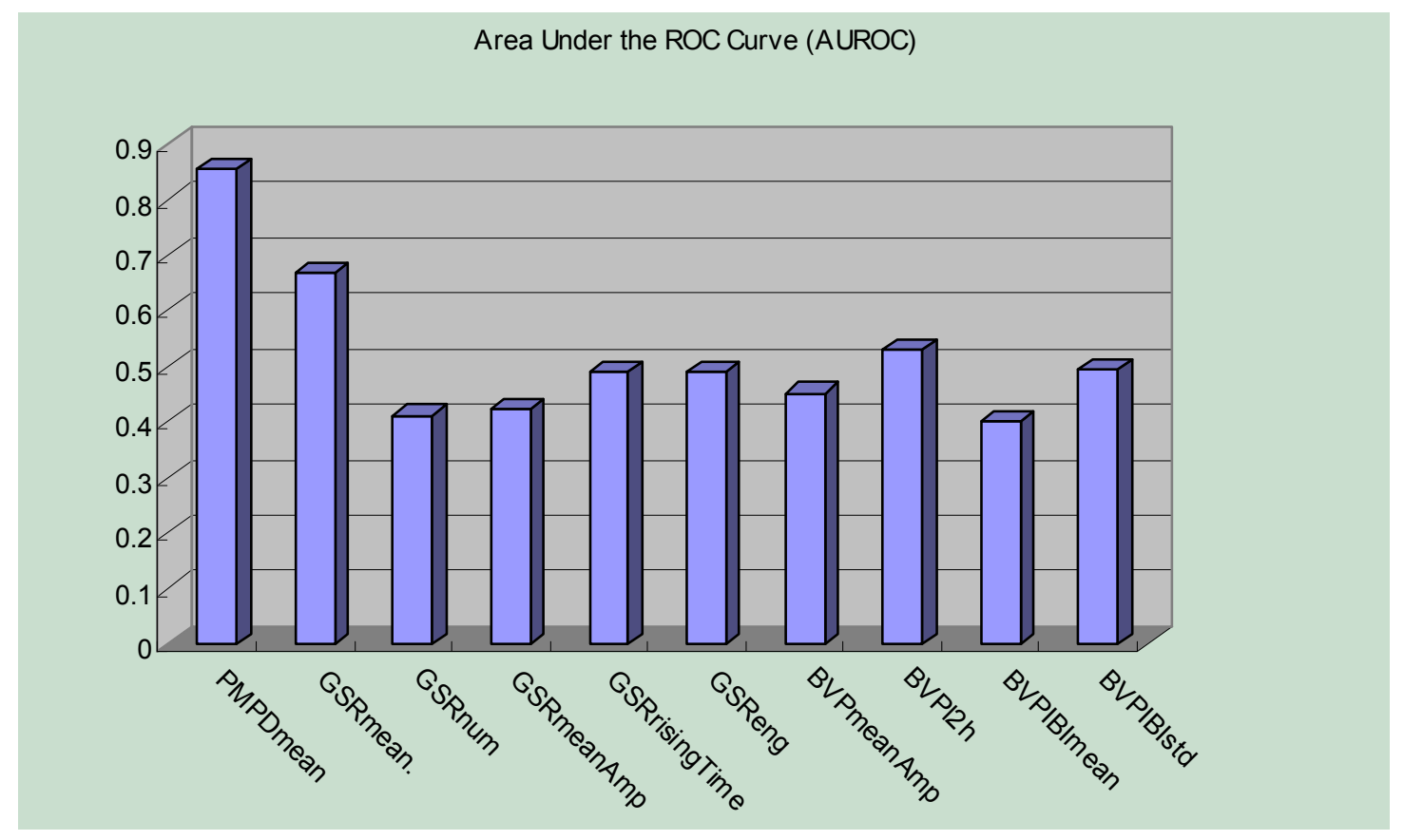

Figure 5-23: Comparison of AUROC values for all 10 features

Figure 5-23 shows a comparison of the area under the ROC curve (AUROC) for the 10 single-feature classifiers. This comparison also emphasizes the higher discriminating power of the "PMPDmean" feature.

\subsection{Summary}

This chapter presented the overall signal processing results obtained from the PD, GSR and BVP signals. Ten features (1 from PD, 5 from GSR, 4 from BVP) were derived and normalized, to be used for stress classification by the SVM machine learning algorithm. Different kernels were used in the implementation of the SVMs used for the affective classification task, and the performance of the classifier with the different kernels, and for the three different evaluation phases ("PMPDmean" only, all 10 features and GSR/BVP features only), were evaluated. The best stress detection accuracy rate is 
achieved by the SVM affective recognition through the PD feature ("PMPDmean"), which was recorded as $77.78 \%$, (the same for all the SVM kernels investigated). The further information of this affective classification is discussed by the followed SVM study.

An important additional stress classification discussion is also provided by the ROC analysis of each single feature. In this form of analysis, also, the highest AUROC value (AUROC $=0.8546$ ) resulted from the ROC curve of "PMPDmean", which strongly suggests that the PD signal should be considered as one of the most powerful physiological factors in future automated affect recognition systems to be developed. This is particularly important because this signal is seldom used in most contemporary affect recognition system prototypes found in the current literature.

An additional important aspect to note in these results is that the encouraging level of affective classification observed from the "PMPDmean" feature was achieved even in spite of the temporary illumination increases introduced during the IC2 and C3 segments of experimentation. This seems to confirm that the AIC system used to process the PD signal may have been effective in minimizing the impact of the PLR effect in these segments. This, in turn, would imply that affective sensing based on the PD signal might be practical even in environments with unconstrained illumination changes. 


\section{CHAPTER 6}

\section{CONCLUSIONS \& FUTURE WORK}

\subsection{Concluding Remarks}

This dissertation has outlined the development of an affective sensing approach to recognize the "stress" states of computer users through the non-invasive monitoring of the pupil diameter (PD) signal. An $\mathrm{H}^{\infty}$ time-varying (HITV) adaptive algorithm has been implemented in an adaptive interference canceller (AIC) to discount the dominant influence of PD variation, the pupillary light reflex (PLR), from a measured PD signal. The output of the AIC, the MPD signal, was post-processed with the application of a non-negative constraint followed by median calculation performed on a sliding window, to generate a PMPD signal, as an indicator of user stress. Specifically, the average value of PMPD in each Stroop segment of the experiment ("PMPDmean" feature) was extracted as an indicator of pupillary affective responses (PAR) due to, for example, subject stress, during the corresponding experimental segment. The approach presented in this dissertation is, therefore, an initial step toward the goal of providing computers with the capability of assessing the affective state of their users, through pupil diameter monitoring, even in the presence of illumination changes.

In the experiment designed for this study, the two affective states to be differentiated were elicited by exposing the subjects to Congruent ("relaxation") and Incongruent ("stress") Stroop color naming trials. Digital signal processing techniques were used to extract a total of 10 features from the 3 monitored physiological signals (PD, GSR and BVP) in each Congruent and Incongruent Stroop segment of the protocol. 
These features were processed by support vector machine (SVM) machine learning classification systems. Using the single feature derived from the processed pupil diameter ("PMPDmean") for the training and testing of the classifier system, an encouraging classification level of $77.78 \%$ was achieved by the SVM. The classification achieved using simultaneously all the 10 features derived from the PD, GSR and BVP signals for the SVM classifier yielded a similar accuracy rate of $76.67 \%$. The last phase of classification evaluation was performed excluding the PD feature from consideration (i.e., using only the 9 features extracted from the GSR and BVP signals). The performance of the classification system trained under this new condition decreased significantly to a $54.44 \%$ accuracy rate. These observations suggest that the $\mathrm{PD}$ is an important physiological signal for affective sensing.

To explore the further stress detection merit of the feature derived from the pupil diameter signal ("PMPDmean"), its single-feature detection power was compared with that of other 9 features extracted from the GSR and BVP signals, simultaneously measured from computer users in this project. The comparison was performed through the receiver operating characteristic (ROC) curves for the individual features. The highest area under the ROC (AUROC) value of 0.8546 was obtained from the analysis of the PD feature, "PMPDmean". This confirms that, indeed, the feature derived from the PD signal has a higher potential for discriminating between the "relaxation" and "stress" subject states than individual features derived from GSR and BVP. All of these results strongly suggest that the PD signal should be considered as one of the most powerful physiological factors to involve in future automated affective recognition systems. Furthermore, this dissertation has proposed and tested a signal processing approach 
targeting the minimization of the impact that environmental lighting variations (within practical application scenarios) would have on this affective sensing modality. Specifically, the proposed AIC system, with the HITV adaptive algorithm, was shown to be effective in minimizing the impact of the pupillary light reflexes triggered by the illumination increases purposefully introduced during the experimental protocol.

With respect to the central questions explored in this dissertation (and posed in Chapter 2), the answers can be provided as:

Q1 Is it feasible to process the pupil diameter signal to minimize the impact of illumination intensity on pupil diameter changes?

Yes. The effect of illumination on measured pupil diameter changes can be modeled and consequently discounted, by an AIC system with the HITV adaptive algorithm, if an independent measurement of the illumination signal is provided.

Q2 Can an Adaptive Interference Canceller (fed with the measured PD and the illumination intensity signal) yield a modified pupil diameter signal that is less impacted by the pupillary light reflex?

Yes. As long as the key conditions for the effective performance of the AIC are met in the situation being addressed, the AIC is able to provide an improved MPD output, under the assumption that the transfer function mediating between illumination changes and corresponding pupil diameter variations can be considered constant within the interval of study.

Q3 Will the PD signal provide a better assessment of the affective status of the subject than other physiological signals, such as GSR and BVP?

Yes. The benefits of the using the PD signal for emotion recognition were confirmed by the encouraging stress detection results through SVM classification of the 
"PMPDmean" feature. The individual feature detector performance assessment by ROC analysis also shows the advantages of affective sensing based on the PD signal, compared with other physiological signals (GSR and BVP).

\subsection{Limitations of Affective Recognition Approaches Based on Physiological}

\section{Signals}

Although affective recognition based on the monitoring of the PD signal circumvents many disadvantages of other modalities, which were introduced in Chapter 2, it still has its own limitations.

An important limitation in affective monitoring through the pupil diameter signal is that, currently, PD signal monitoring is totally reliant on the proper setup and operation of the eye tracker (in this dissertation, the TOBII T60 system). Improper operation of the eye tracker system, even on a transient basis, could impede the affective sensing process. Similarly, most physiological-based affective sensing approaches require that the proper interface be established (and maintained) between the sensors used and the body (or eyes) of the subject. Fulfilling this requirement may limit the comfort level of the subject, particularly if the restrictions will be imposed on him/her for a long time. For example, in

the experiments performed for this dissertation, the subject was required to sit still during the whole experimental period, minimizing movements of his/her head, as well as activity of his/her left hand. It can be hoped that future sensor technology may provide interface means that will be more robust and more natural to the user.

Additionally, it should also be acknowledged that individual temperament and personality affect the expression of emotions. Extroverts tend to be more expressive than 
introverts. Expressive patterns also depend on gender, context, and social and cultural expectation. To reach the goal of a person-independent emotion recognition system for universal usage, robust signal processing and pattern recognition techniques that specifically account for those individual differences may need to be developed.

\subsection{Future Work}

The development of this dissertation research has revealed the success of affective sensing through the non-invasive monitoring of the PD signal. However, the proposed approach can be enhanced by exploring additional research avenues in the future. These directions will be summarized in the following paragraphs.

$\checkmark$ Real-time implementation of the affect sensing system (through PD monitoring);

In this dissertation, the analyses developed are all performed off-line, on previously recorded data. However, an affective computer in real life would be expected to operate in real (or near-real) time, interactively tuning its disposition to its user, on the basis of the assessment of his/her affective state. This desired functionality calls for on-line, real-time implementation of the affective sensing capabilities. The results from this study provide a successful starting point for the development of affect sensing that may, in the future, be implemented for on-line performance.

\section{$\checkmark$ Integration of other affective sensing modalities;}

Besides physiological signals, such as PD, GSR and BVP, there are many other factors that can be considered to reflect the affective states of computer users, such as face expressions, speech activity etc.. This research has only investigated one promising direction to pursue the affective recognition goal (monitoring of pupil diameter signal). 
Some of the other modalities could be integrated together in the future to seek a better emotion recognition performance.

$\checkmark$ Add self-report stress evaluations to the experiments;

In the experiments conducted for this research, the only two affective states evaluated were "relaxation" and "stress". In reality, the degree of stress effectively induced in the subjects during the experiment may not be uniform. The use of a properly formulated self-assessment questionnaire, administered to the subject immediately after the completion of the experimental session could, perhaps, be used to qualify, or even "weight" the stress level that a given Incongruent Stroop segment effectively represented. It might then be possible to incorporate this additional information in the classifier training procedure, to enhance the classification capability ultimately achieved by these systems.

$\checkmark$ Evaluation of the system with larger subject pools.

The system proposed here was developed (i.e., training of the learning classifier) and evaluated with signals recorded from 30 participants. For each subject, six feature vectors, i.e., six classification samples, were extracted. This corresponds to 180 classification samples in total that were used to train and test in the SVM machine learning system. This data sample size should be expanded by collecting data from a larger pool of subjects, so that the resulting learning classifiers may be better able to properly recognize the affective status of subjects who did not participate in their training. 


\section{REFERENCES}

[1] J. Raskin, Humane Interface: New Directions for Designing Interactive Systems. Boston: Addison-Wesley, 2000.

[2] R. W. Picard, Affective Computing: MIT, 1997.

[3] P. Ekman and W. Friesen, Manual for the Facial Action Coding System, Palo Alto: Consulting Psychology Press, 1978.

[4] N. L. Etcoff and J. J. Magee, "Categorical perception of facial expressions," Cognition, vol. 44, pp. 227-240, 1992.

[5] K. A. a. B.-B. N. De Silva P. R., "Towards unsupervised detection of affective body posture nuances," in 1st Int. Conf. Affective Computing and Intelligent Interaction (ACII), Beijing, China, 2005, pp. 32-40.

[6] A. Kapur, V. K. A., N. L. Etcoff, G. Tzanetakis, and P. F. Driessen," Gesture-based affective computing on motion capture data," in 1st Int. Conf. Affective Computing and Intelligent Interaction (ACII'), Beijing, China, 2005, pp. 1-8.

[7] N. Campbell, "Perception of affect in speech - towards an automatic processing of paralinguistic information in spoken conversation," in International Conference on Spoken Language Processing, Jeju, 2004.

[8] F. Dellaert, T. Polzin, and A. Waibel, "Recognizing emotion in speech," in International Conference on Spoken Language Processing Philadelphia, PA, 1996, pp. 1970-1973.

[9] V. A. Petrushin, "Emotion recognition in speech signal: experimental study, development and application," in International Conference on Spoken Language Processing, Beijing, 2000.

[10] R. Brunelli and D. Falavigna, "Person identification using multiple cues," IEEE Trans. On Pattern Analysis and Machine Intelligence, vol. 12, pp. 955-966, 1995.

[11] E. S. Bigun, J. Bigun, B. Duc, and S. Fischer, "Expert conciliation for multimodal person authentication systems using Bayesian statistics," in Proc. International Conference on Audio and Video-Based Biometric Person Authentication (AVBPA), Crans-Montana, Switzerland, 1997, pp. 291-300.

[12] S. Chaffar and C. Frasson, "The emotional conditions of learning," in Proc. Int. FLAIRS Conference, Clearwater, FL, USA, 2005, pp. 201-206.

[13] S. Chaffar and C. Frasson, "Predicting learner's emotional response in intelligent distance learning systems," in Proc. 19th Int. FLAIRS Conference, Melbourne, FL, USA, 2006, pp. 383-388. 
[14] F. Jones and J. Bright, Stress - Myth, Theory and Research: Prentice Hall, 2001.

[15] R. D. Clover, T. Abell, and L. A. Becker, "Family functioning and stress as predictors of influenza B infection," Journal of Family Practice, vol. 28, pp. 535-539, 1989.

[16] N. M. Graham, R. M. Douglas, and P. Ryan, "Stress and acute respiratory infection," American Journal of Epidemiology, vol. 124, pp. 389-401, 1986.

[17] A. Barreto, "Non-intrusive physiological monitoring for affective sensing of computer users," in Human-Computer Interaction New Developments, Kikuo Asai, Ed. Vienna: I-Tech, 2008, pp. $85-100$.

[18] E. Hudlicka, "To feel or not to feel: the role of affect in human-computer interaction," International Journal of Human-Computer Studies, vol. 59, pp. 1-32, 2003.

[19] K. Hugdahl, Psychophysiology: The Mind-Body Perspective: Harvard University Press, 2001.

[20] F. H. Martini, Fundamentals of Anatomy and Physiology, 5 ed., 2000.

[21] J. I. Lacey, "Psychophysiological approaches to the evaluation of psychotherapeutic process and outcome," in Research in Psychotherapy, vol. 1, E. A. Rubinstein and M. B. Padoff, Eds. Washington, DC: American Psychological Association, 1959, pp. 160-208.

[22] J. Cacioppo and L. Tassinary, "Inferring psychological significance from pshysiological signals,," American Psychologist, vol. 45, pp. 16-28, 1990.

[23] J. T. Cacioppo, G. G. Berntson, and B. L. Andersen, "Psychophysiological approaches to the evaluation of psychotherapeutic process and outcome, 1991: contributions from social psychophysiology," Psychological Assessment, vol. 3, pp. 321-336, 1991.

[24] J. Beatty and B. Lucero-Wagoner, "The pupillary system. ," in Handbook of Psychophysiology, 2 ed., Cacioppo, Tassinary, and Berntson, Eds.: Cambridge University Press, 2000, pp. 142-162.

[25] S. R. Steinhauer, G. J. Siegle, R. Condray, and M. Pless, "Sympathetic and parasympathetic innervation of pupillary dilation during sustained processing," International Journal of Psychophysiology vol. 52, pp. 77-86, 2004.

[26] P. C. Bressloff and C. V. Wood, "Spontaneous oscillations in a nonlinear delayed-feedback shunting model of the pupillary light refelex," Physical Review vol. 58, pp. 3597-3605, 1998. 
[27] J. Keener and J. Sneyd, Mathematical Physiology. New York: Springer-Verlag, 2001.

[28] C. Darwin, The Expression of the Emotions in Man and Animals. Chicago: University of Chicago Press, 1965.

[29] E. H. Hess, The Tell-tale Eye: How Your Eyes Reveal Hidden Thoughts and Emotions. New York: Van Nostrand Reinhold, 1975.

[30] W. W. Grings and M. E. Dawson, Emotions and Bodily Responses a Psychophysiological Approach: Academic Press, 1978.

[31] J. G. Kennedy, R. S. Williams, M. C. Dunlap, W. P. Brannan, and J. R. Brannan, "Eye movement indices of mental workload," Acta Psychologica, vol. 75, pp. 75-89, 1990.

[32] T. Partala and V. Surakka, "Pupil size variation as an indication of affective processing," Int. J. of Human-Computer Studies, vol. 59, pp. 185-198, 2003.

[33] R. F. A. Eric Granholm and A. J. Sarkin, "Pupillary responses index cognitive resource limitations," Psychophysiology, vol. 33, pp. 457-461, 1996.

[34] B. W. H. Ludtke, M. Adler, and F. Schaeffel, "Mathematical procedures in data recording and processing of pupillary fatigue waves," Vision Research, vol. 38, pp. 2889-2896, 1998.

[35] C. Darrow, "The galvanic skin reflex (sweating) and blood pressure as preparatory and facilitative functions," Psychological Bulletin, vol. 33, pp. 73-94, 1936.

[36] R. S. Lazarus, J. C. Speisman, and A. M. Mordkoff, "The relationships between autonomic indicators of psychological stress: heart rate and skin conductance," Psychosomatic Medicine, vol. 25, pp. 19-31, 1963.

[37] A. L. Krogstad, M. Elam, T. Karlsson, and B. G. Wallin, "Arteriovenous anastomoses and the thermoregulatory shift between cutaneous vasoconstrictor and vasodilator reflexes," J. Auton. Nerv. Syst., vol. 53, pp. 215-222, 1995.

[38] H. Selye, Selye's Guide to Stress Research: Van Nostrand Reinhold Company, 1980.

[39] N. H. Frijda, "Studies in emotion and social interaction," in The Emotions, chapter Physiology of Emotion Cambridge: Cambridge University Press, 1986, pp. 124-175.

[40] P. J. Lang, "The emotion probe: studies of motivation and attention," American Psychologist, vol. 50, pp. 372-385, 1995. 
[41] R. S. Lazarus and S. Folkman, Stress, Appraisal and Coping. New York: Springer, 1984.

[42] W. B. Cannon, Bodily Changes in Pain, Hunger, Fear and Rage. New York: Appleton, 1929.

[43] J. E. Mcgrath, Social Psychologic Factors in Stress. New York: Holt. Rinehart and Winston, 1970.

[44] T. Cox, Stress Research and Stress Management: Putting Theory to Work. Sffolk, UK 1993.

[45] L. F. Berkman, "The role of social relations in health promotion," Psychosomatic Medicine, vol. 57, pp. 245-254, 1995.

[46] A. Mathews and C. MacLeod, "Discrimination of threat cues without awareness in anxiety states," Journal of Abnormal Psychology, vol. 95, pp. 1-8, 1986.

[47] P. Rani, J. Sims, R. Brackin, and N. Sarkar, "Online stress detection using psychophysiological signal for implicit human-robot cooperation," Robotica, vol. 20, pp. 673-686, 2002.

[48] R. D. Ward and P. H. Marsden, "Affective computing: problems, reactions and intentions," Interacting with Computers, vol. 16, pp. 707--713, 2004.

[49] B. Widrow and S. D. Stearns, Adaptive Signal Processing. Englewood Cliffs, NJ: Prentice-Hall, 1985.

[50] B. Hassibj and T. Kailath, " $\mathrm{H}^{\infty}$ adaptive filtering," in Proc. Int. Conf. Acoustics, Speech, Signal Processing, 1995, pp. 945-952.

[51] S. Puthusserypady, " $\mathrm{H}^{\infty}$ adaptive filters for eye blink artifact minimization from electroencephalogram," IEEE Signal Processing Letters, vol. 12, pp. 816-819, 2005.

[52] L. Stark, "Stability, oscillations, and noise in the human pupil servomechanism," in Proc. I.R.E, 1959, pp. 1925-1939.

[53] R. J. Clarke, H. Zhang, and P. D.R.Gamlin, "Characteristics of the pupillary light reflex in the alert rhesus monkey," Journal of Neurophysiol, vol. 89, pp. 3179-3189, 2003.

[54] A. R. Bentivoglio, S. B. Bressman, E. Cassetta, D. Carretta, P. Tonali, and A. Albanese, "Analysis of blink rate patterns In normal subjects," Movement Disorders, vol. 12, pp. 1028-1034, 1997. 
[55] J. T. Cacioppo, L. G. Tassinary, and G. Berntson, Handbook of Psychophysiology, 2nd ed.: Cambridge University Press, 2000.

[56] P. Y. Ktonas, "Automated spike and sharp wave (SSW) detection," in Methods of Analysis of Brain Electrical and Magnetic Signals, EEG Handbook (revised series), 1987, pp. 211-241.

[57] C. J. C. Burges, "A tutorial on support vector machines for pattern recognition," Data Mining and Knowledge Discovery, vol. 2, pp. 121-167, 1998.

[58] T. Joachims, "Text categorization with support vector machines: learning with many relevant features," in Proc of thel0th European Conference on Machine Learning, Chemnitz, Germany, 1998, pp. 137-142.

[59] E. Osuna, R. Freund, and F. Girosi, "Training support vector machines: an application to face detection," in Proc of the IEEE Conf. Computer Vision and Pattern Recognition, 1997, pp. 130-136.

[60] B. Scholkopf, K. K. Sung, C. J. C. Burges, F. Girosi, P. Niyogi, T. Poggio, and V. Vapnik, "Comparing support vector machines with Gaussian kernels to radial basis function classifiers," IEEE Transactions on Signal Processing, vol. 45, pp. 2758-2765, 1997.

[61] M. Adjouadi and M. Ayala, "Introducing neural studio: an artificial neural networks simulator for educational purposes," Computers in Education Journal, vol. 14, pp. 33-40, 2004.

[62] M. Adjouadi and A. Zong, "Multidimensional pattern recognition and classification of white blood cells using support vector machines," Journal of Particle and Particle Systems Characterization, vol. 22, pp. 107-118, 2005.

[63] B. Efron and R. Tibshirani, An Introduction to the Bootstrap. New York, London: Chapman and Hall, 1993.

[64] R. Collobert and S. Bengio, "Svmtorch: support vector machines for large-scale regression problems," J. Machine Learn, vol. 1, pp. 143-160, 2001.

[65] T. Joachims, Making Large-scale SVM Learning Practical: MIT-Press, 1999.

[66] P. Pavlidis, I. Wapinski, and W. S. Noble, "Support vector machine classification on the web," Bioinformatics, vol. 20, pp. 586-587, 2004.

[67] C.-C. Chang and C.-J. Lin, LIBSVM: a Library for Support Vector Machines, 2001. 
[68] J. R. Stroop, "Studies of interference in serial verbal reactions," Journal of Experimental Psychology, vol. 18, pp. 643-662, 1935.

[69] J. Zhai and A. Barreto, "Significance of pupil diameter measurements for the assessment of affective state in computer users," Biomedical Sciences Instrumentation, vol. 42, pp. 495-500, 2006.

[70] P. Renaud and J. P. Blondin, "The stress of stroop performance: physiological and emotional responses to color-word interference, task pacing, and pacing speed," International Journal of Psychophysiology, vol. 27, pp. 87-97, 1997.

[71] J. R. Stroop, "Interference in serial verbal reactions," Journal of Experimental Psychology, vol. 18, pp. 643-661, 1935.

[72] R. M. Stern, W. J. Ray, and K. S. Quigley, Psychophysiological Recording, 2 ed.: Oxford University Press, 2001.

[73] A. Todoroki and N. Hana, "Luminance change method for cure monitoring of GFRP," Key Engineering Materials, vol. 321-323, pp. 1316-1321, 2006.

[74] M. E. Dawson, A. M. Schell, and D. L. Filion, "The electrodermal system," in Handbook of Psychophysiology, 2 ed., J. T. Cacioppo, T. L.G., and B. G.G., Eds. Cambridge,UK: Cambridge University Press, 2000.

[75] R. W. Picard and J. Klein, "Computers that recognize and respond to user emotion: theoretical and practical implications," Interacting with Computers, vol. 14, pp. 141-169, 2002. 
APPENDICES 


\section{APPENDIX A - IRB FORM}

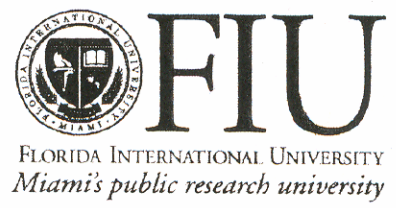

InE aproved

Dare: $10 / 02 / 08$

Ni. $\quad 100807-01$

\section{CONSENT TO PARTICIPATE IN A RESEARCH STUDY}

\section{TITLE: DIGITAL SIGNAL PROCESSING APPROACH FOR AFFECTIVE SENSING \\ THROUGH PUPIL DIAMETER MONITORING}

Thank you for participating in this Affective Computing study! You are being asked to participate in the research project listed above to be conducted at Florida International University with Dr. Armando Barreto as the principal investigator. The co-investigator of this study is Ying Gao and she is a student in the Department of Electrical Engineering, FIU. The purpose of investigation is to design and develop a DSP approach for affective sensing through pupil diameter monitoring. Your participation will require about 40 minutes of your time. We are trying to measure and collect data from real time pupil diameter measurement. The study will include about 30 people.

In this research project, we attempt to detect people's affective states. In order to do this, we need to monitor the pupil diameter changes continuously using the DSP approach we propose to develop. This newly designed approach is built in the form of adaptive noise canceling system, which is based on two sets of input signals. The primary input is the pupil diameter signal recorded by an eye tracking system and the reference input is a luminance intensity signal obtained by an illumination measurement system.

The sensors used in this experiment are safe for the participants. The Galvanic Skin Response sensor where you will place two fingers is a battery-driven device, which will measure the moisture in your skin. A $9 \mathrm{~V}$ battery also drives the photoplethysmograph sensor you will gently clip to one of your fingers, and it emits very low power infrared light to estimate the amount of blood in your finger's capillary bed. A luminance sensor will be placed on your forehead to record the illumination intensity changes of the surroundings. This sensor will not be in electrical

University Park • Miami, Florida 33199 
contact with your skin. A desk-mounted TOBII T60 eye tracking system that will be capturing the image of your eye also uses very low power infrared light to illuminate your eye, so that the location and the size of your pupil can be continuously measured. None of the sensors used in the experiment are invasive, and they will not cause any form of sensation in your fingers.

In the experiment, you will be asked to wear the sensors on and remain seated in front a computer screen, for about 40 minutes. During this time, all the lights in the room will be kept on. However, the illumination level of the experimental surrounding will be changed repeatedly by turning lamps on and off. In this part, a lamp dimmer will be used to introduce illumination , changes. Simultaneously, you will be instructed automatically by the computer to participant in a Stroop Color-Word Interference Test, which is implemented with Macromedia Flash. In this test, you will be shown words that designate color names. Regardless of the meaning of the word shown, you need to click one of the five buttons shown on the screen, to indicate the font color used in the word shown.

If you decide to be a part of the study we will tell you what day and time to come to the Digital Signal Processing Lab, Room EC-3970, in the Engineering Center of Florida International University. It is not foreseen that the experiment may cause you any harm or severe discomfort. However, if you get upset or feel discomfort during the experiment, you may ask to take a break. There is no cost or payment to you as a subject. You will not get any direct benefit from being in the study. However, your help will give us valuable data information in order to verify our system and our efforts for the proposed study. Your participation will be identified by a random code number, not by your name. All of the data collected in the experiment is private and will not be shared with anyone unless required by law. Your data will be compared to the data of the other subjects. We will present the research results as a group. You may ask questions about the study at any time. If you choose not to participate no one will be upset with you. You may also choose to stop your participation before your finish the experiment.

If you would like more information about this research after you are done, you can contact Dr. Armando Barreto, Associate professor in the Departments of Electrical and Computer 
Engineering / Biomedical Engineering at 305-348-3711 or me at 305-348-6072. If you would like to talk with someone about being a subject in this study you may contact Dr. Patricia Price, the Chairperson of the FIU Institutional Review Board at 305-348-2618 or 305-348-2494.

Your signature below indicates that all questions have been answered to your liking. You are aware of your rights and you would like to be in the study.

Signature of Participant

Printed Name

Date

I have explained the research procedure, subject rights and answered questions asked by the participant. I have offered him/her a copy of this informed consent form.

Signature of Witness

Date 


\section{APPENDIX B - MATLAB CODE FOR PD, GSR, BVP SIGNALS}

\section{PROCESSING \& FEATURE EXTRACTION}

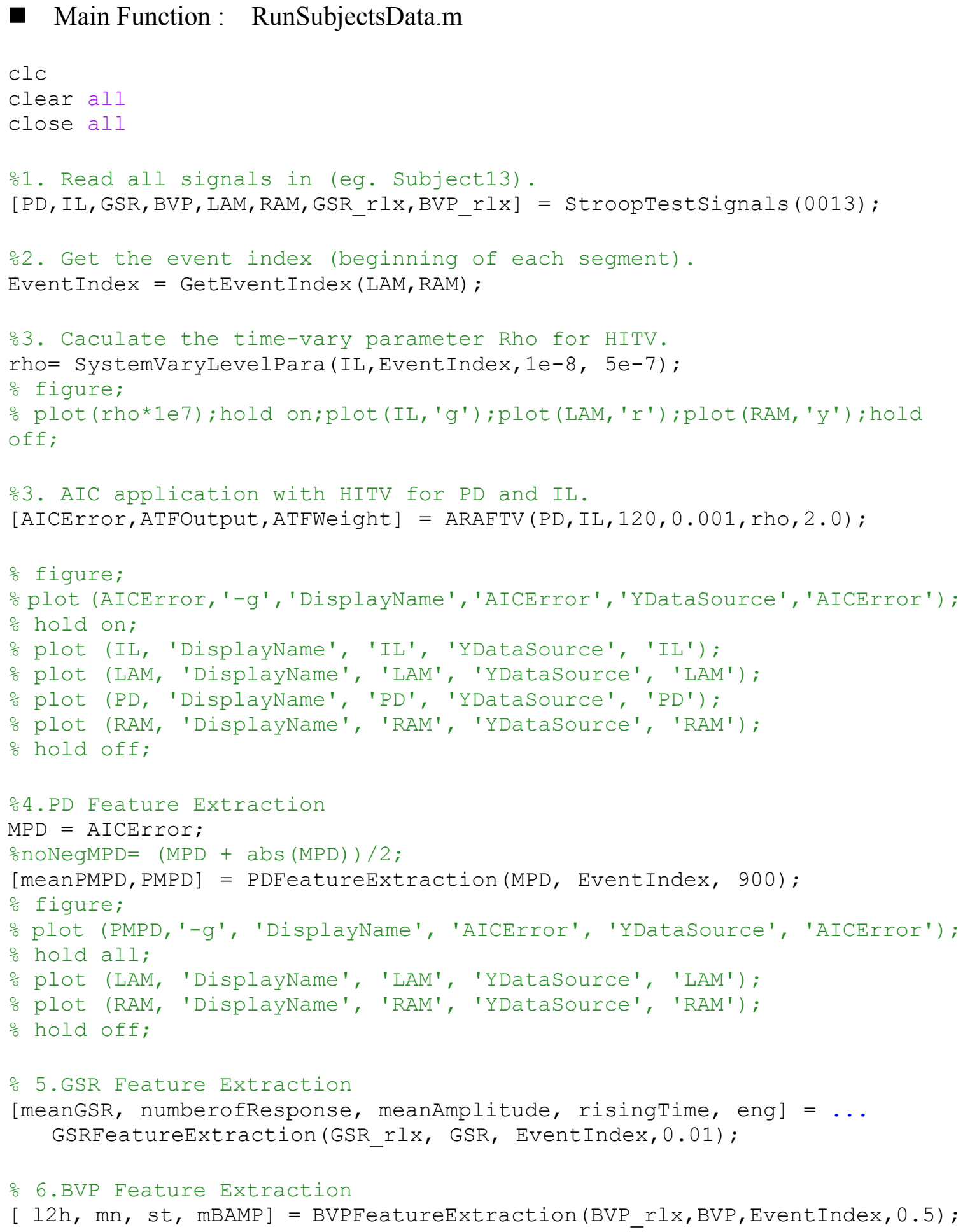




\section{- Function: StroopTestSignal.m}

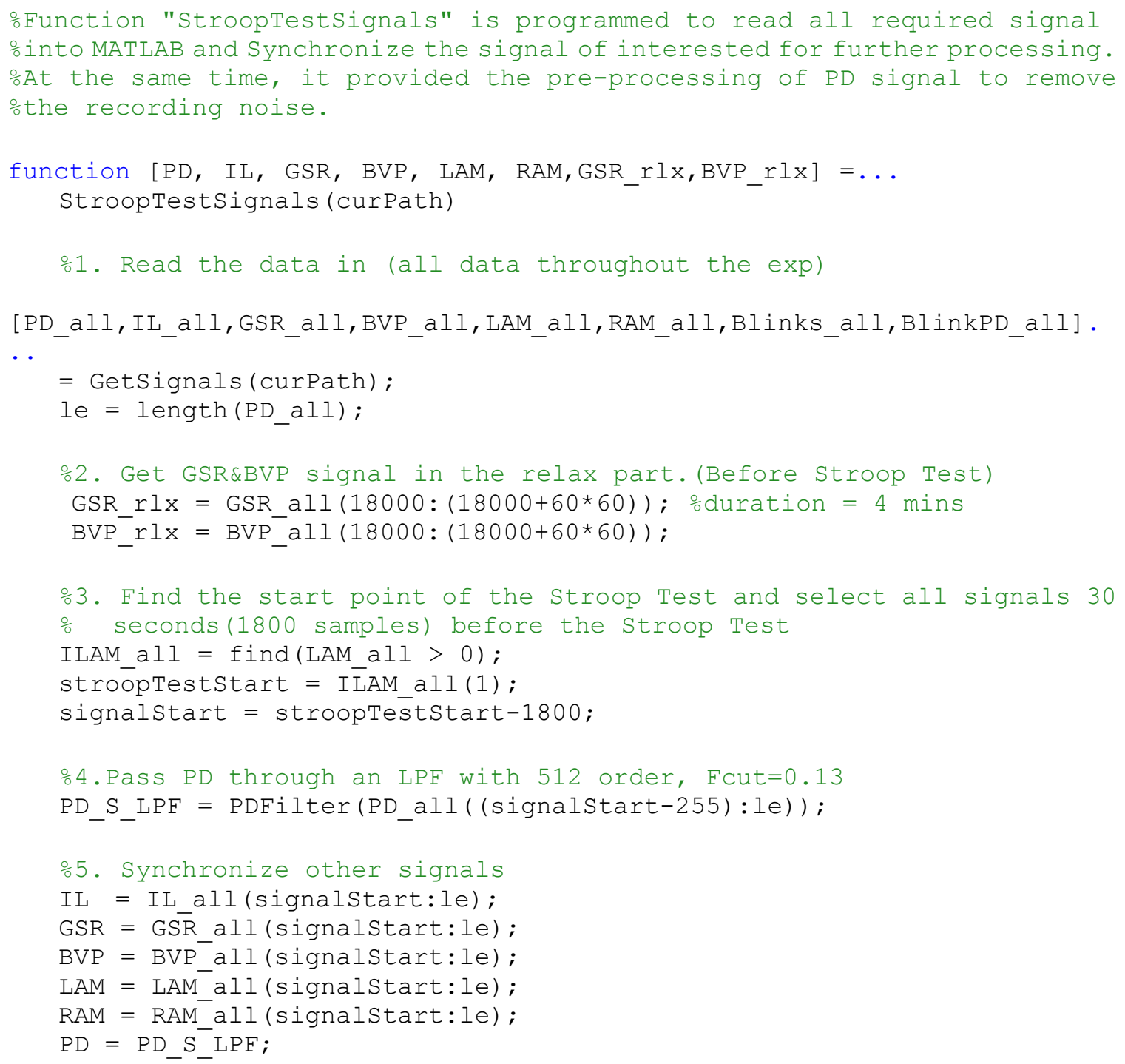

\section{- Function: GetSignals.m}

\%Function "GetSignals" reads the recorded data in Matlab, downsample DAQ oto $60 \mathrm{~Hz}$, and process the blinks in PD.

function [PD, IL, GSR, BVP, LAM, RAM, Blinks,BlinkPD] = GetSignals(curPath)

\%1. Read data from DAQ and downsample them from $360 \mathrm{~Hz}$ to $60 \mathrm{~Hz}$

[IL360 RAu360 LAu360 GSR360 BVP360] =

ReadBinaryFileDaq (curPath) ; $\mathrm{f} s=360 \mathrm{~Hz}$

IL60= downsample $($ IL360,6); 


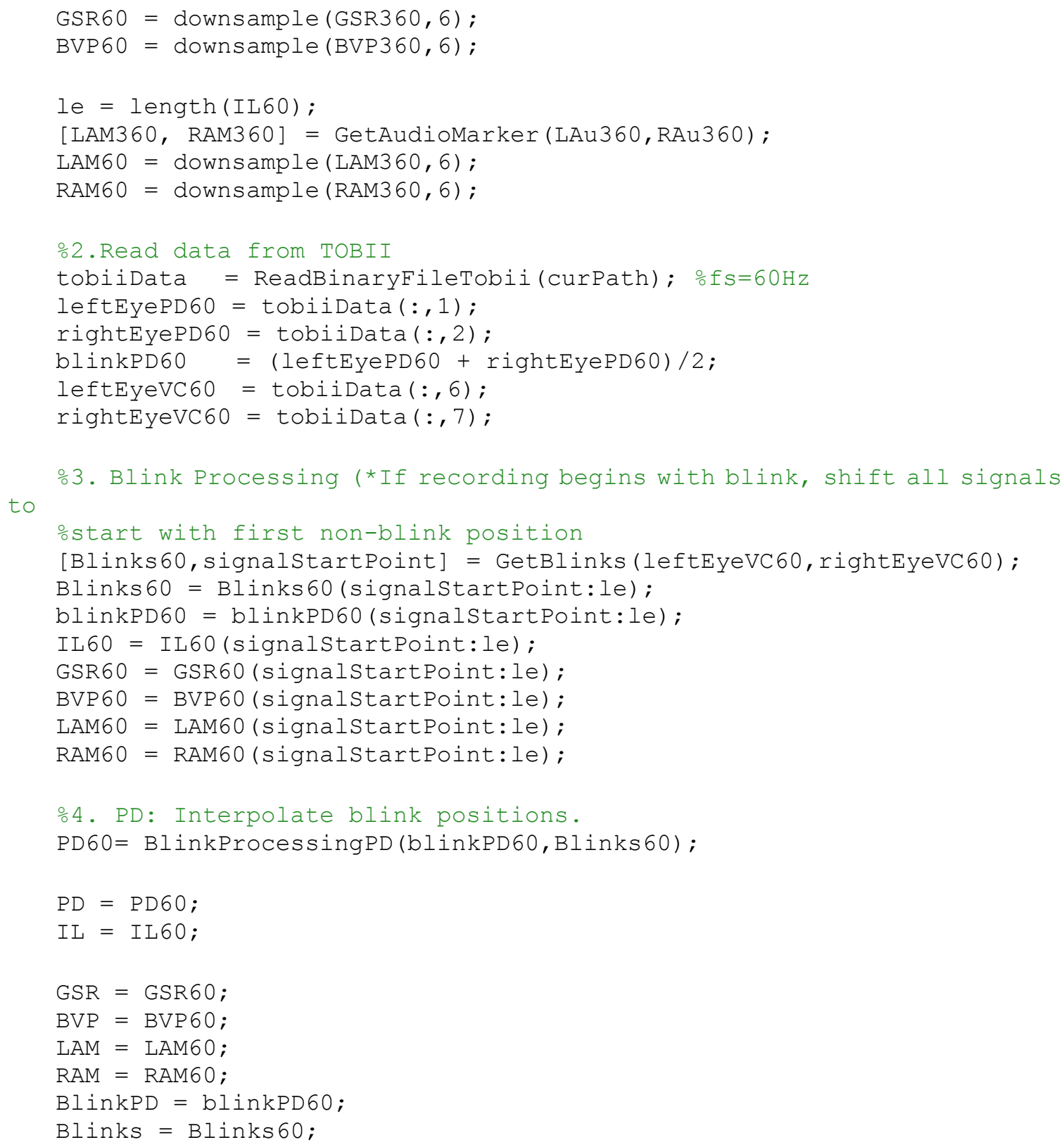

\section{- Function: ReadBinaryFileDaq.m}

oRead DAQ data in to matlab workspace.

function [IL RAu LAu GSR BVP DAQ]= ReadBinaryFileDaq(curPath)

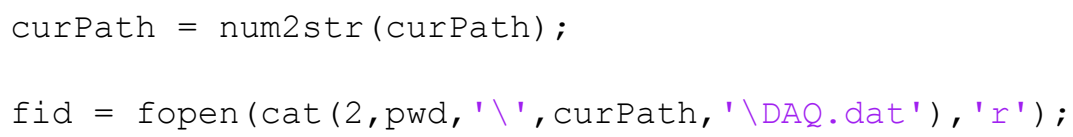




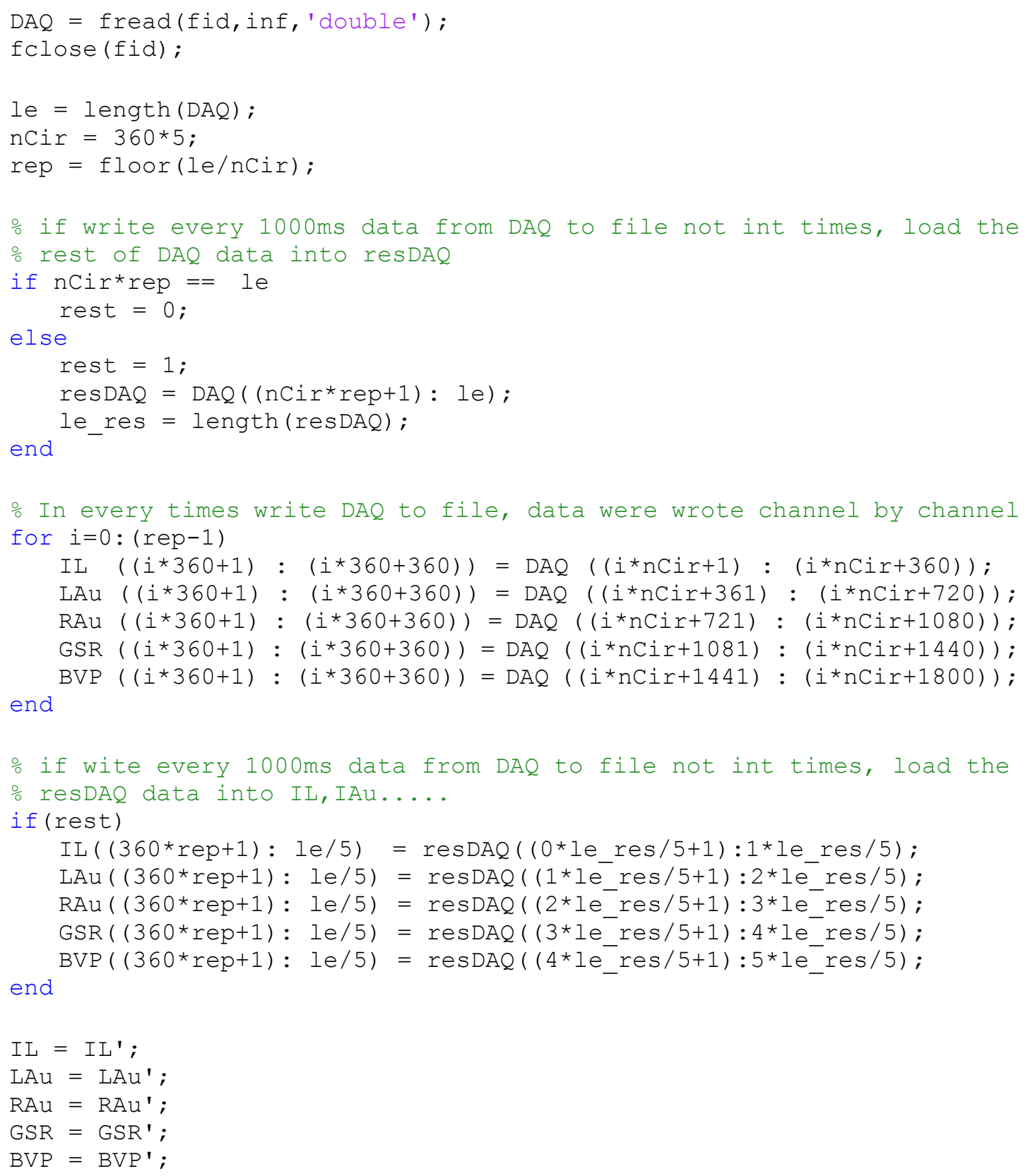

\section{- Function: ReadBinaryFileTobii.m}

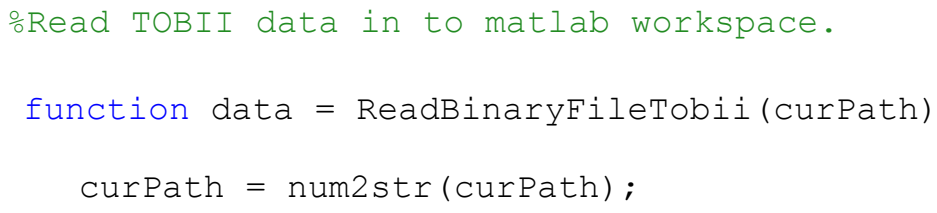




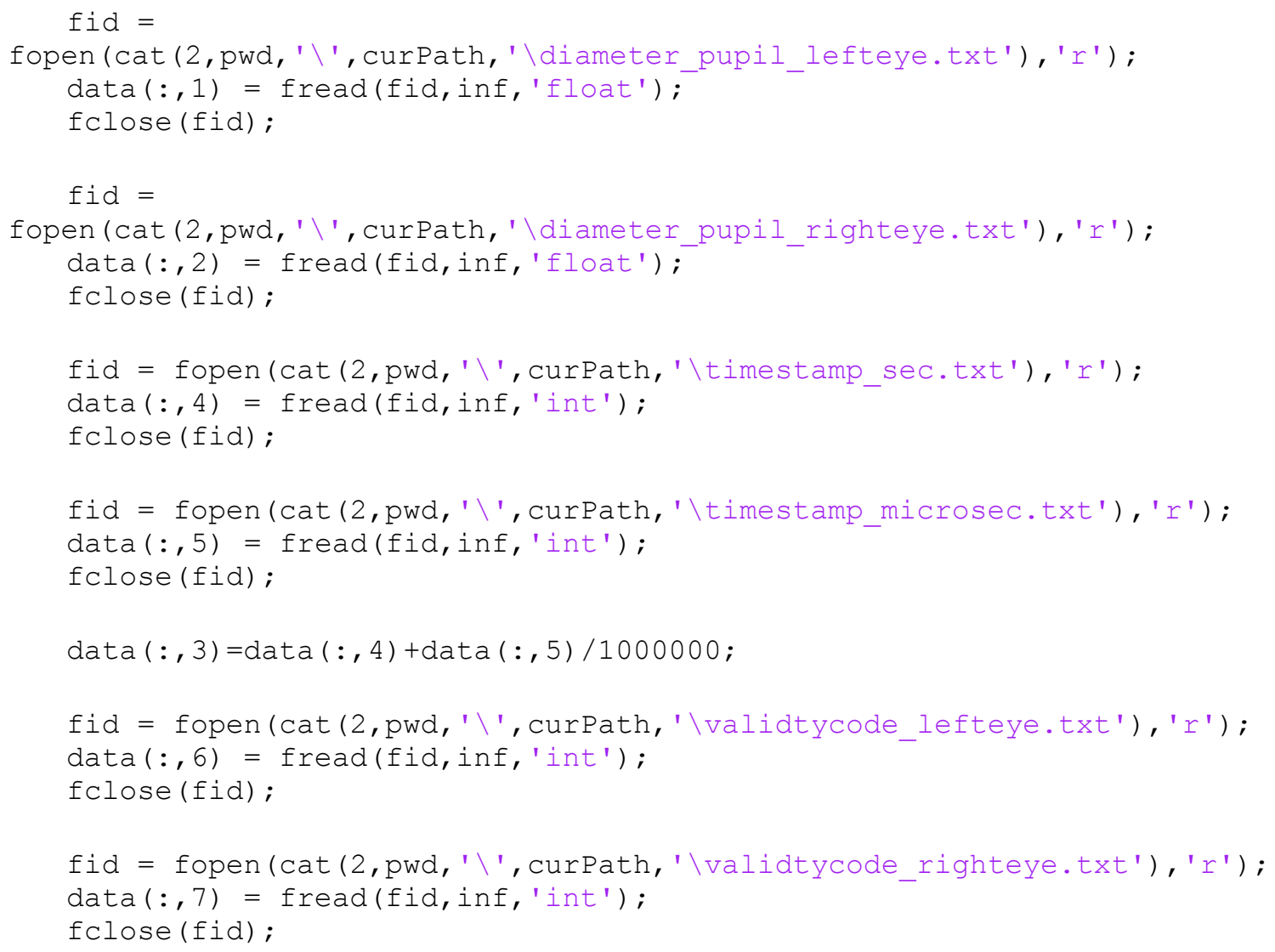

\section{- Function: GetAudioMarker.m}

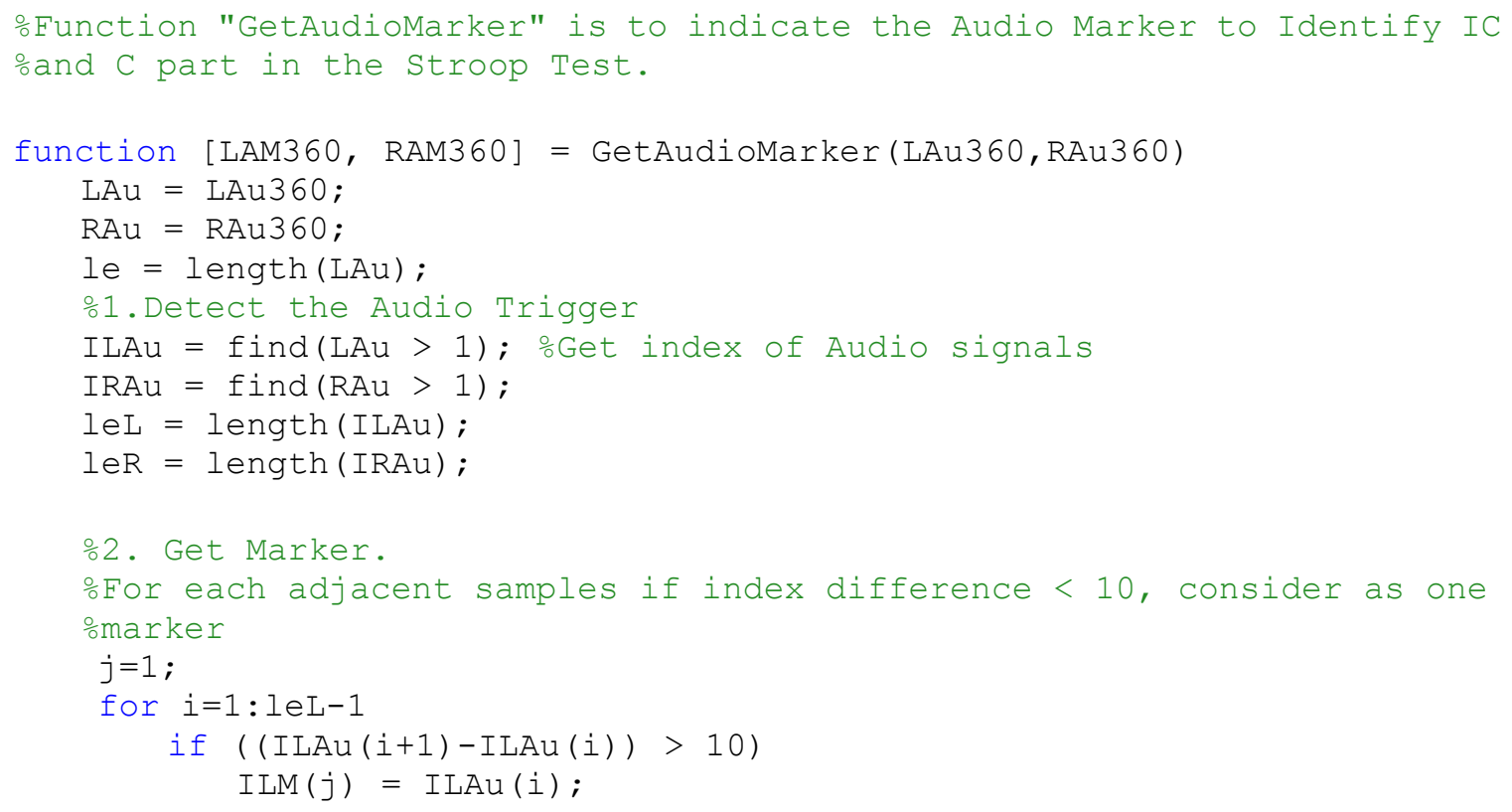




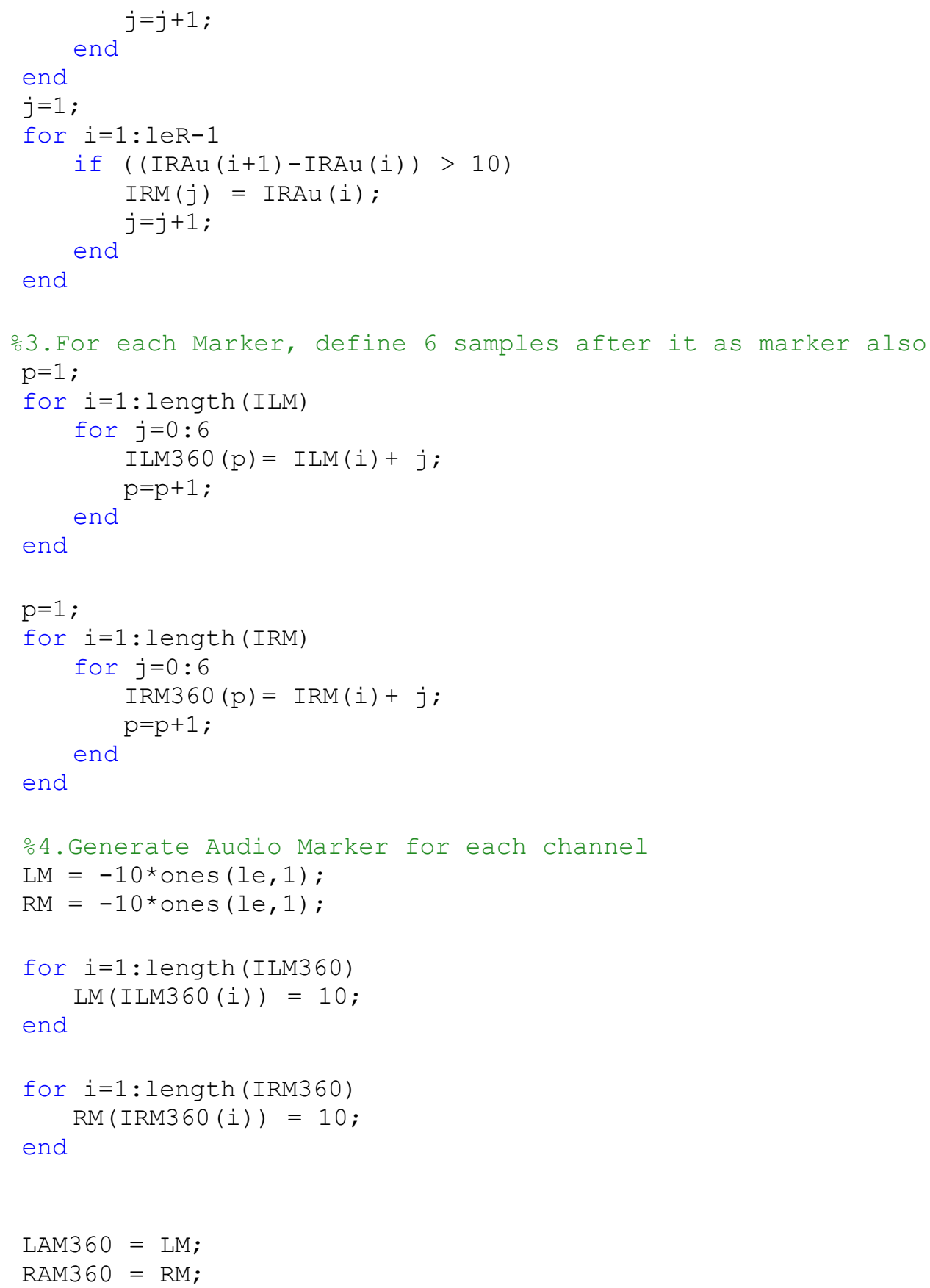

- Function: GetBlinks.m

\%Function "GetBlinks" is programmed to Get the blink positions in the PD osignal and at the same time resize all the other signals

function [Blinks, signalStartPoint] = GetBlinks(leftEyeVC,rightEyeVC) 


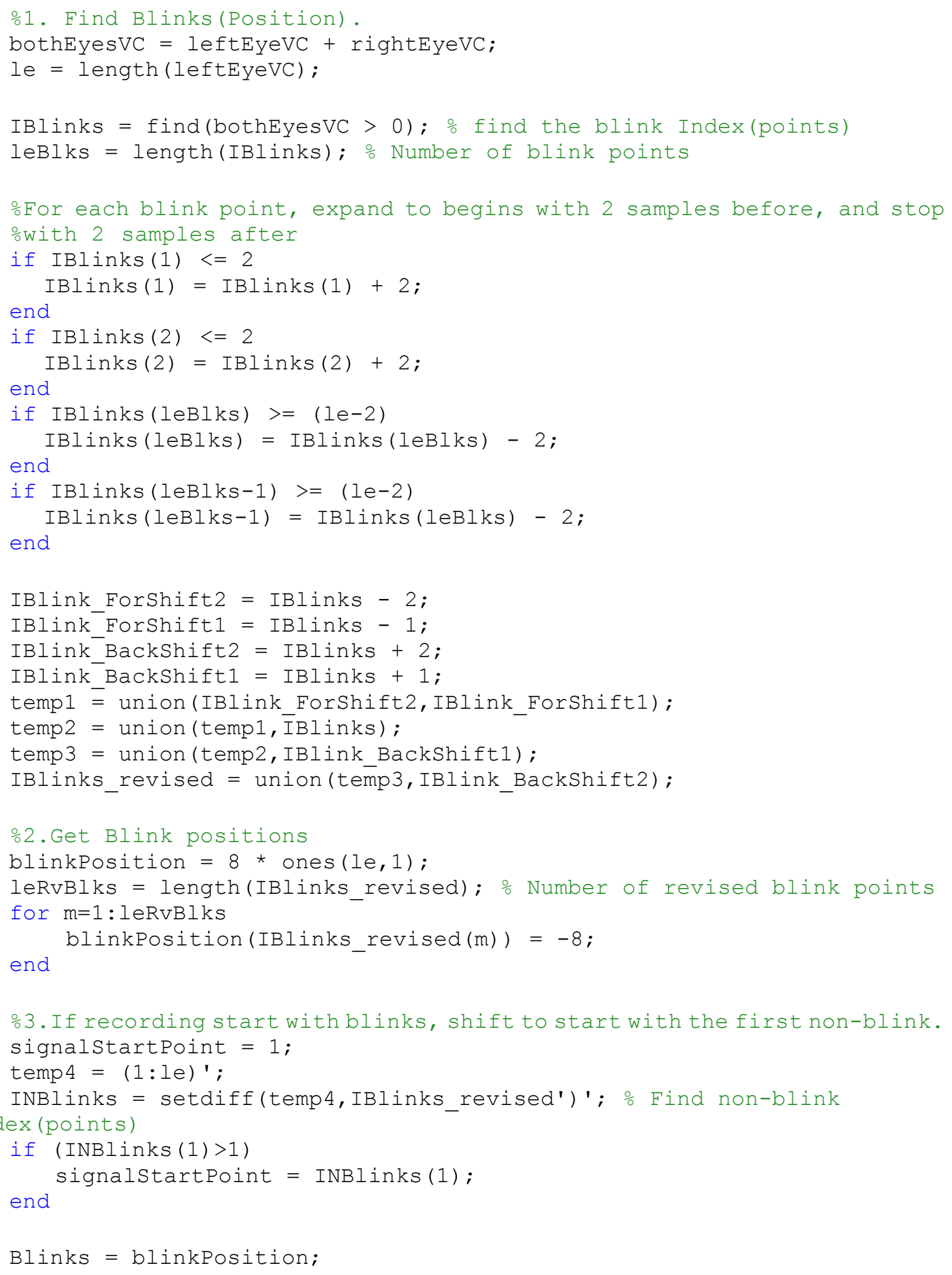




\section{- Function: PDFilter.m}

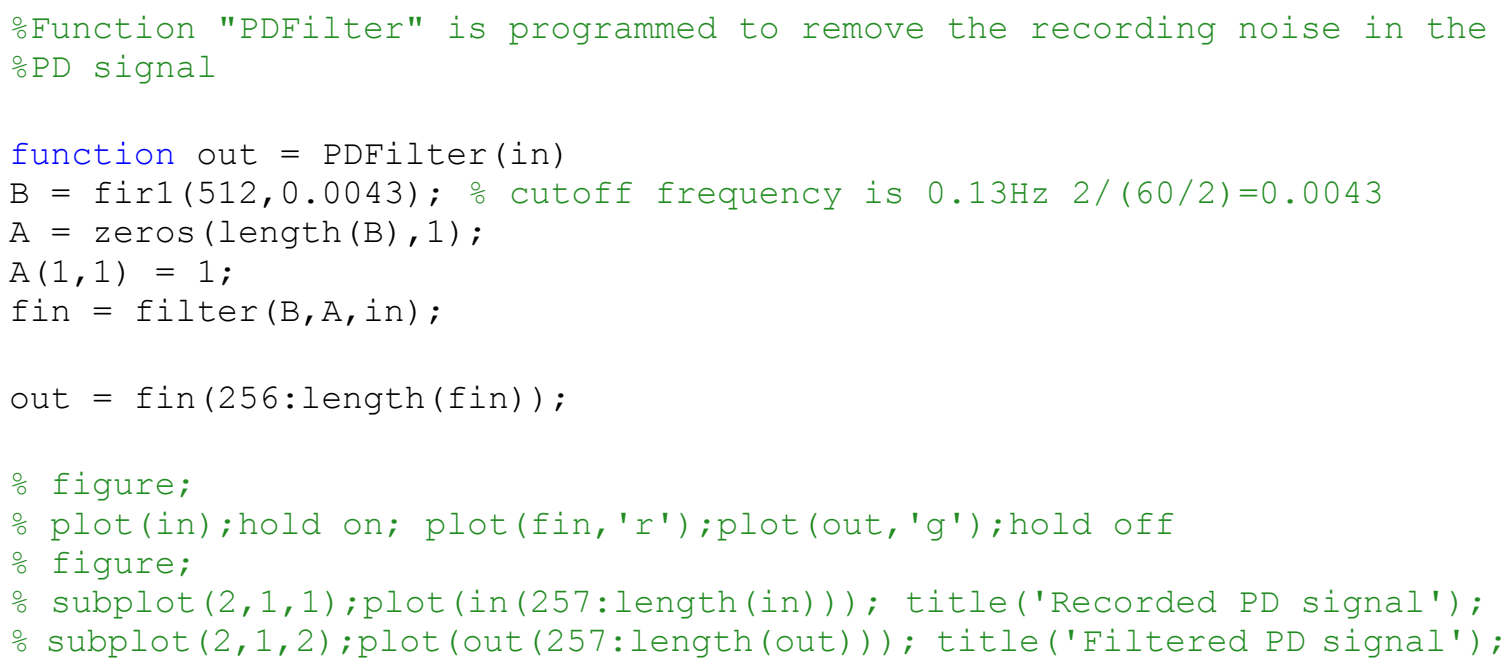

\section{- Function: GetEventIndex.m}

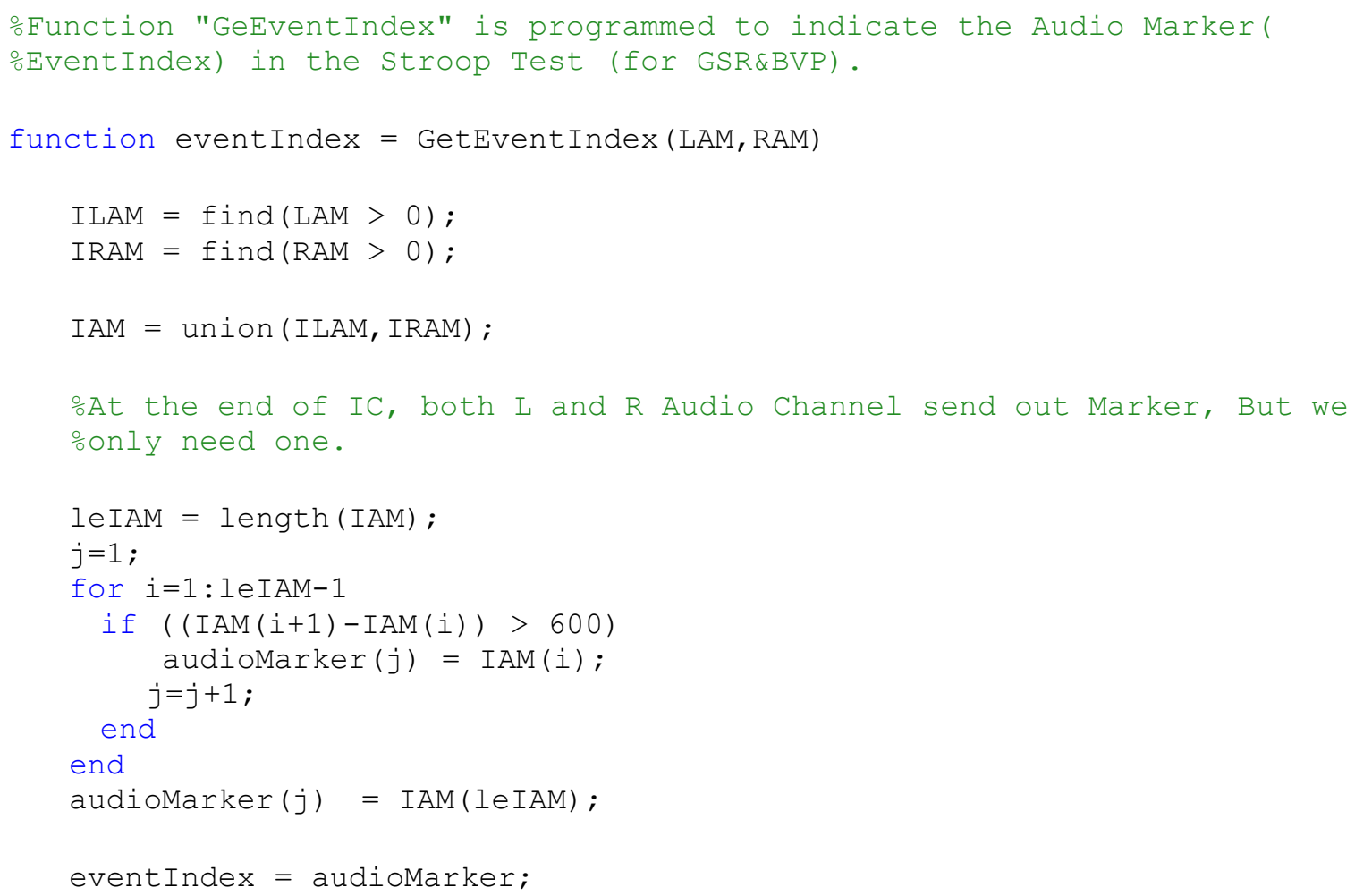




\section{- Function: SystemVaryLevelPara.m}

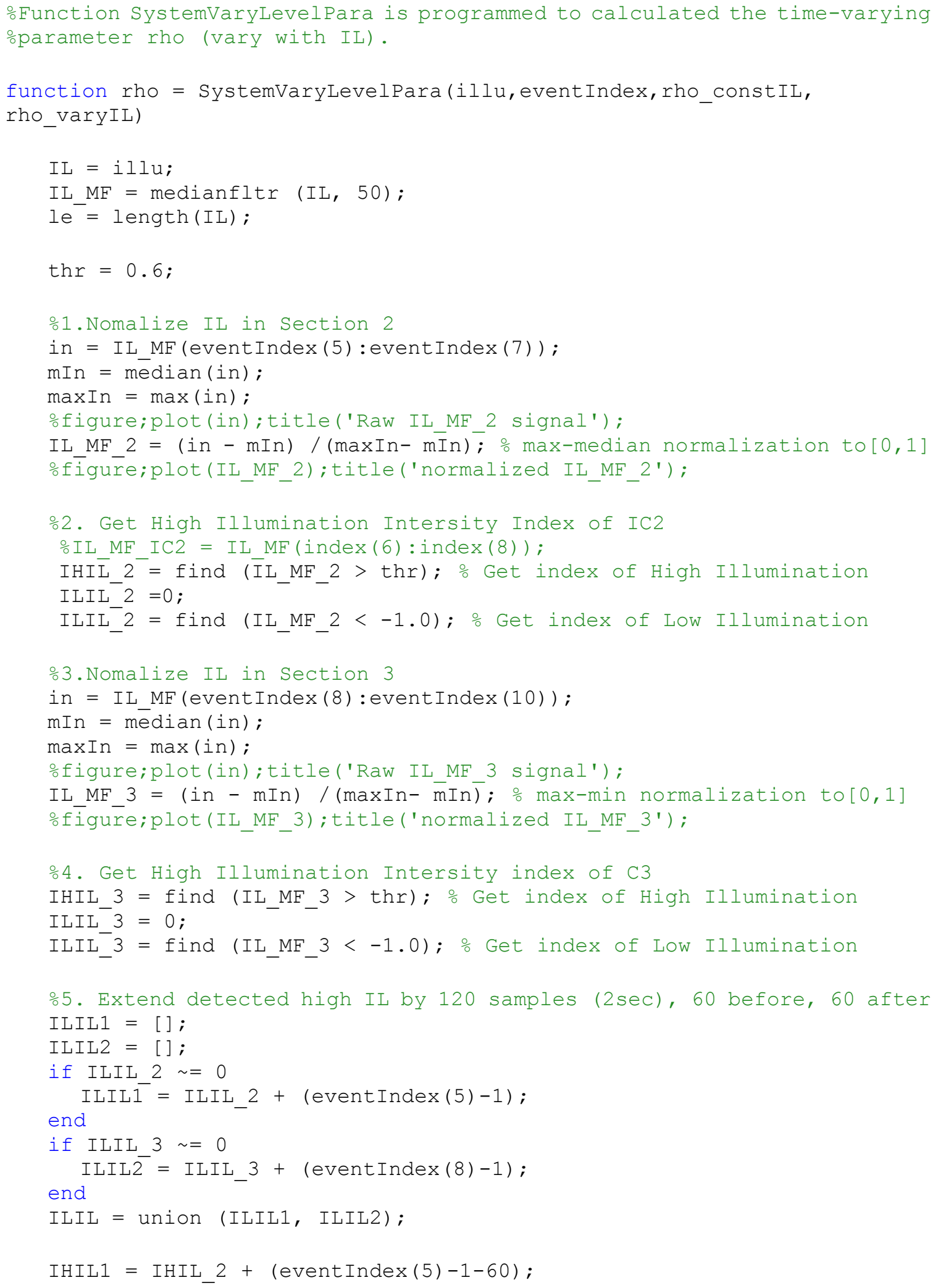




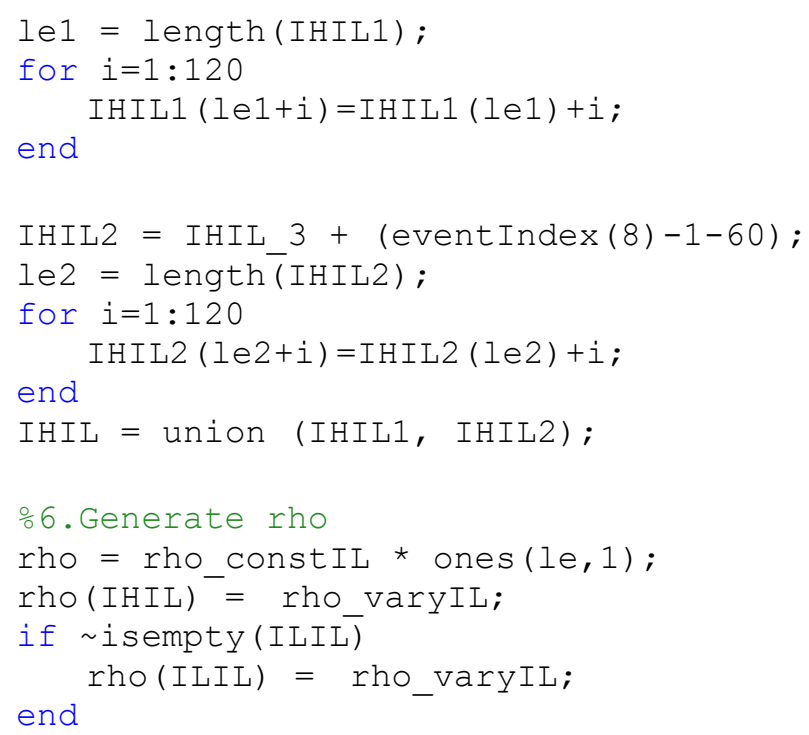

- Function: ARAFTV.m

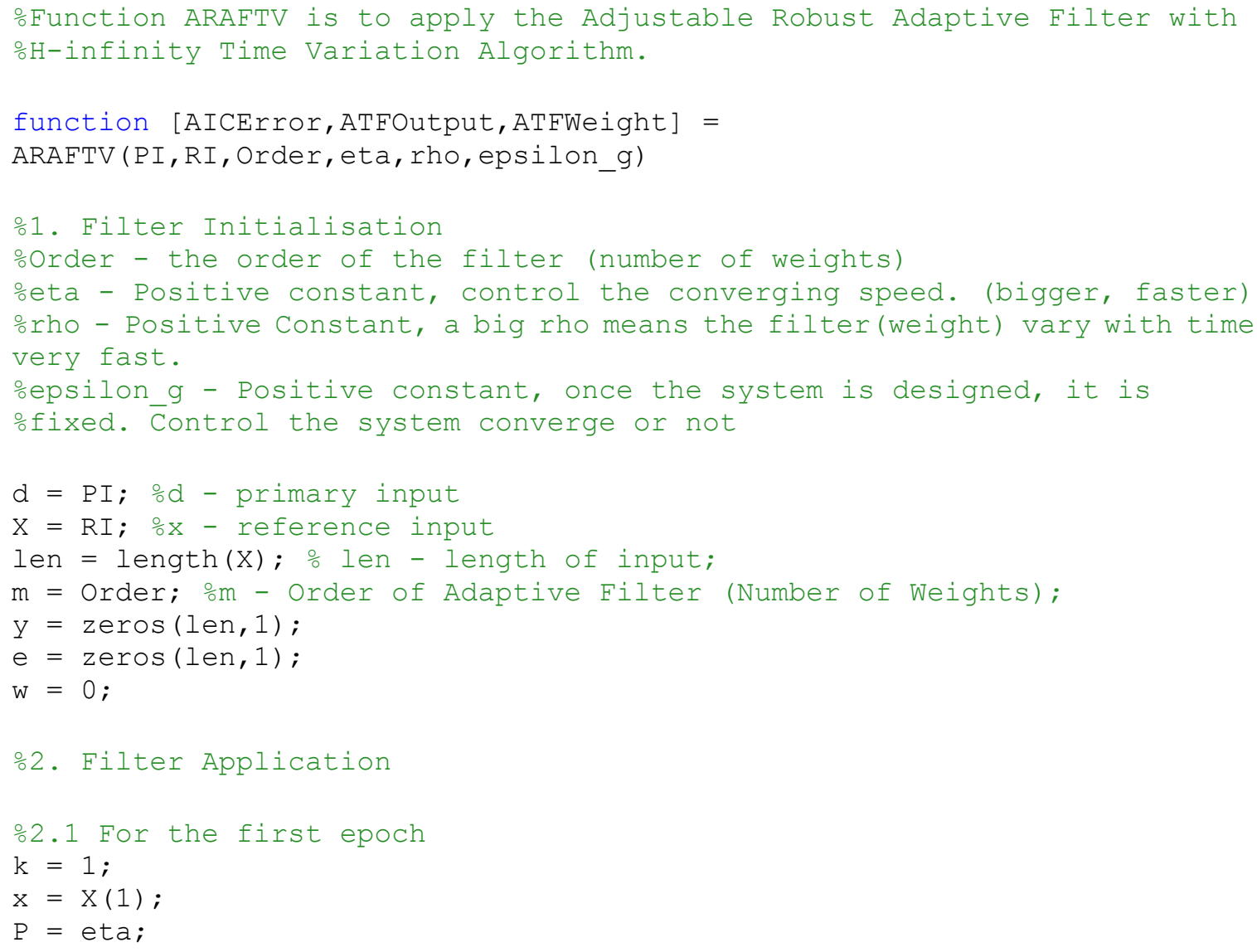




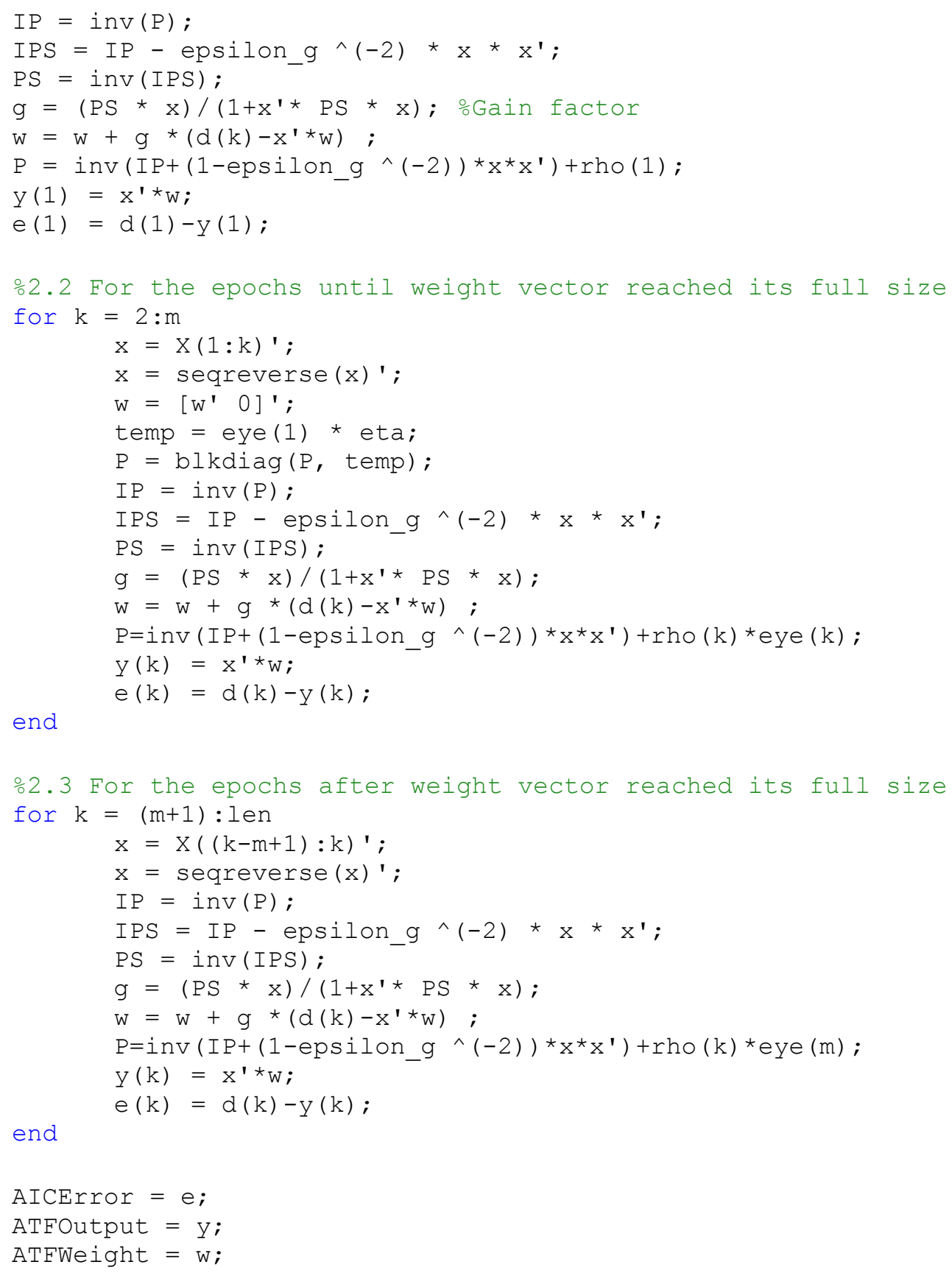

\section{- Function: PDFeatureExtraction.m}

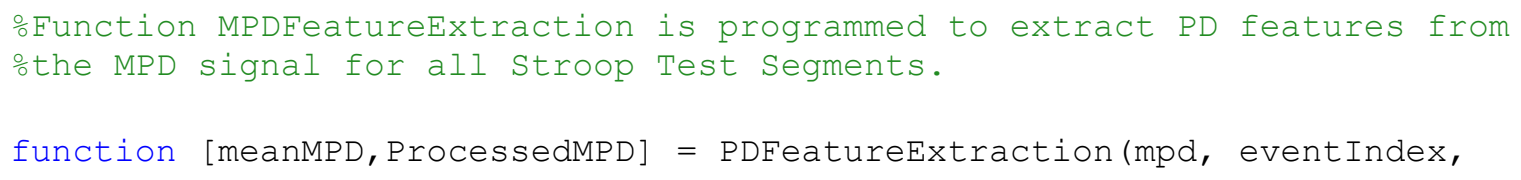




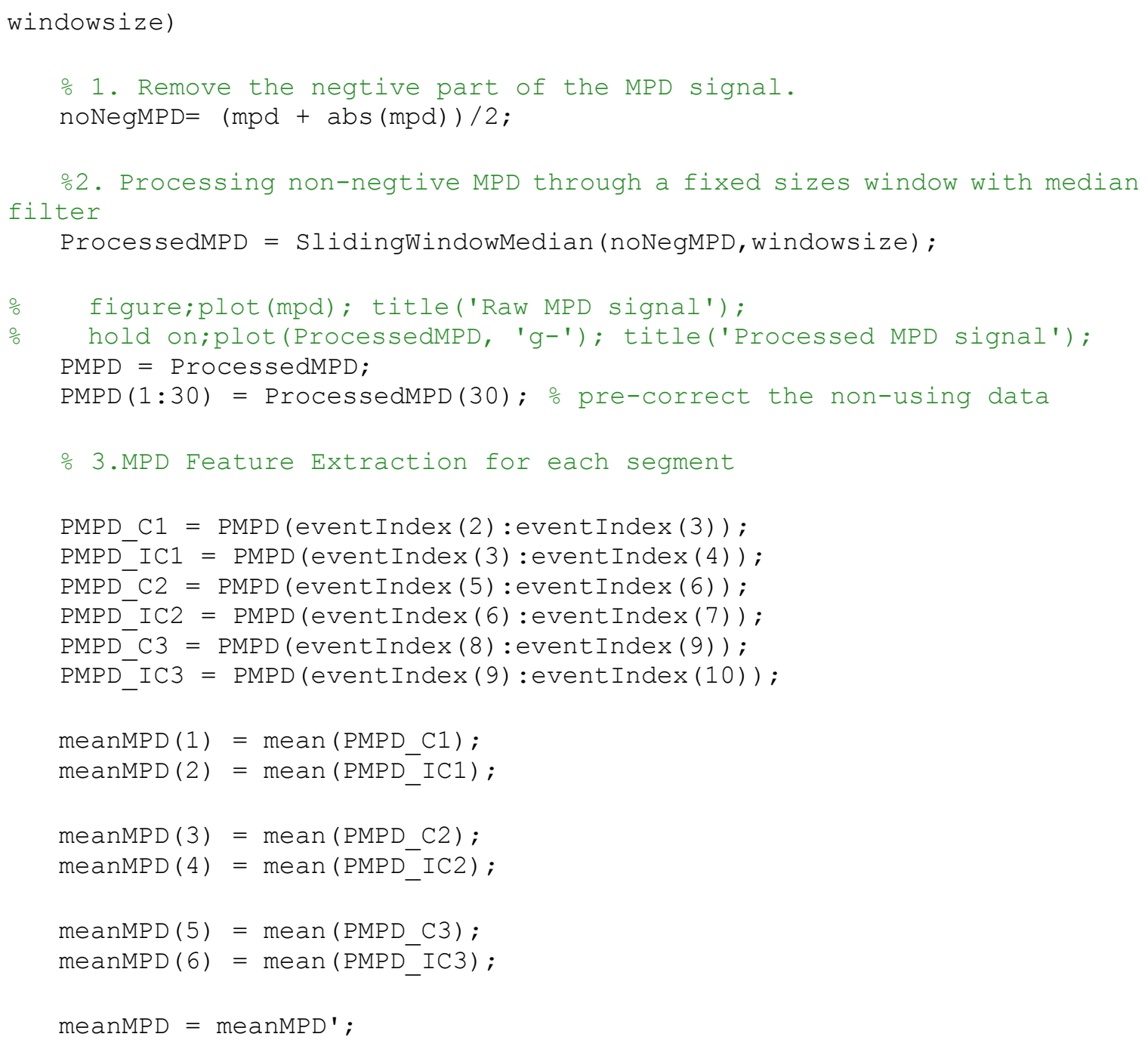

\section{- Function : SlidingWindowMedian.m}

oFunction SlidingWindowMean is used to calculate the input signal's median ovalue with sliding window.

function median_sliding = SlidingWindowMedian(signal,windowSize)

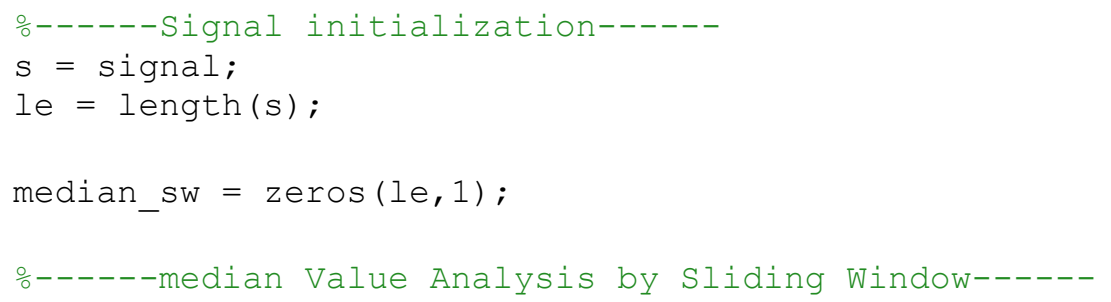




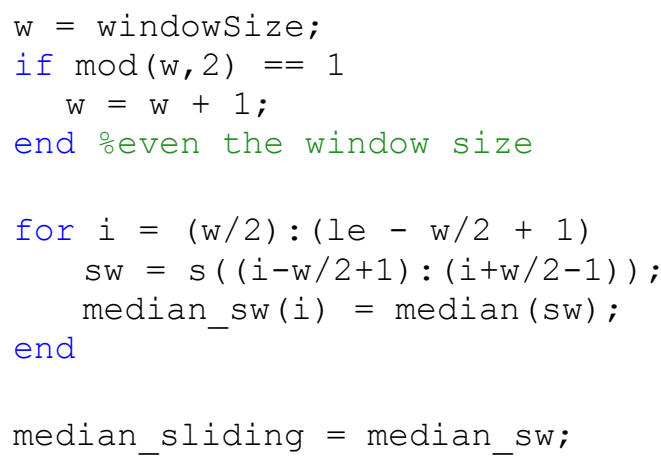

\section{- Function: GSRFeatureExtraction.m}

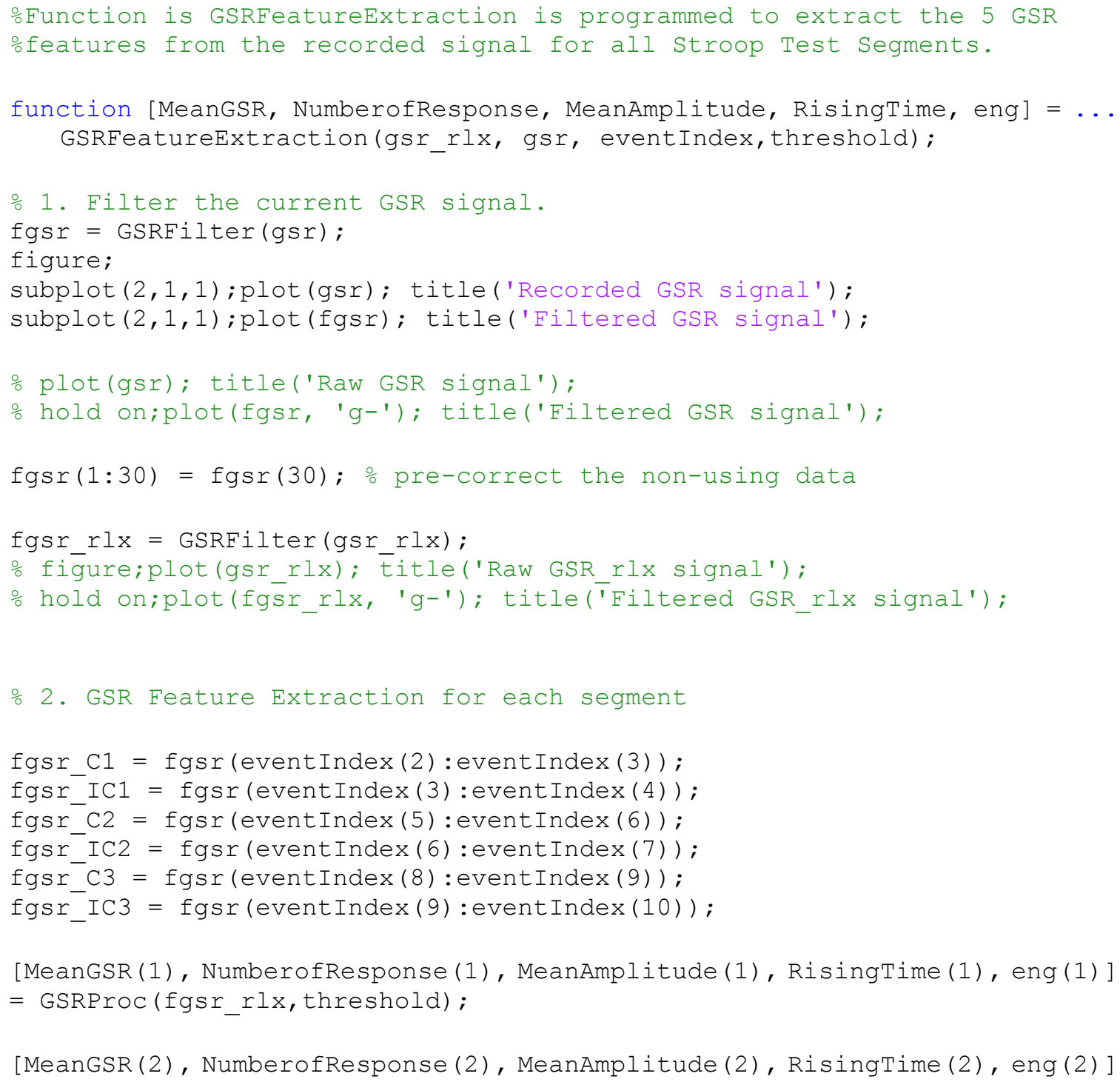




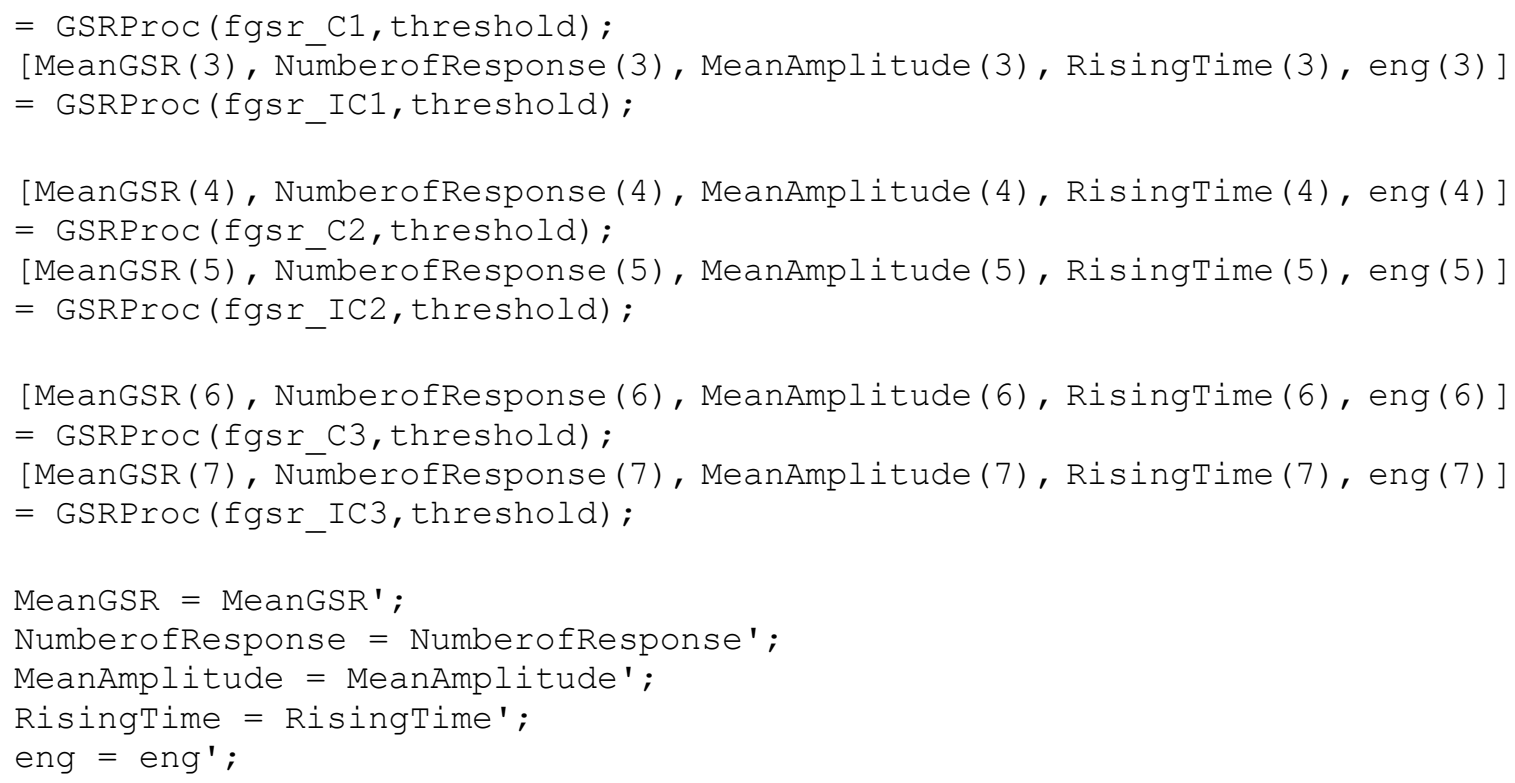

\section{- Function: GSRFilter.m}

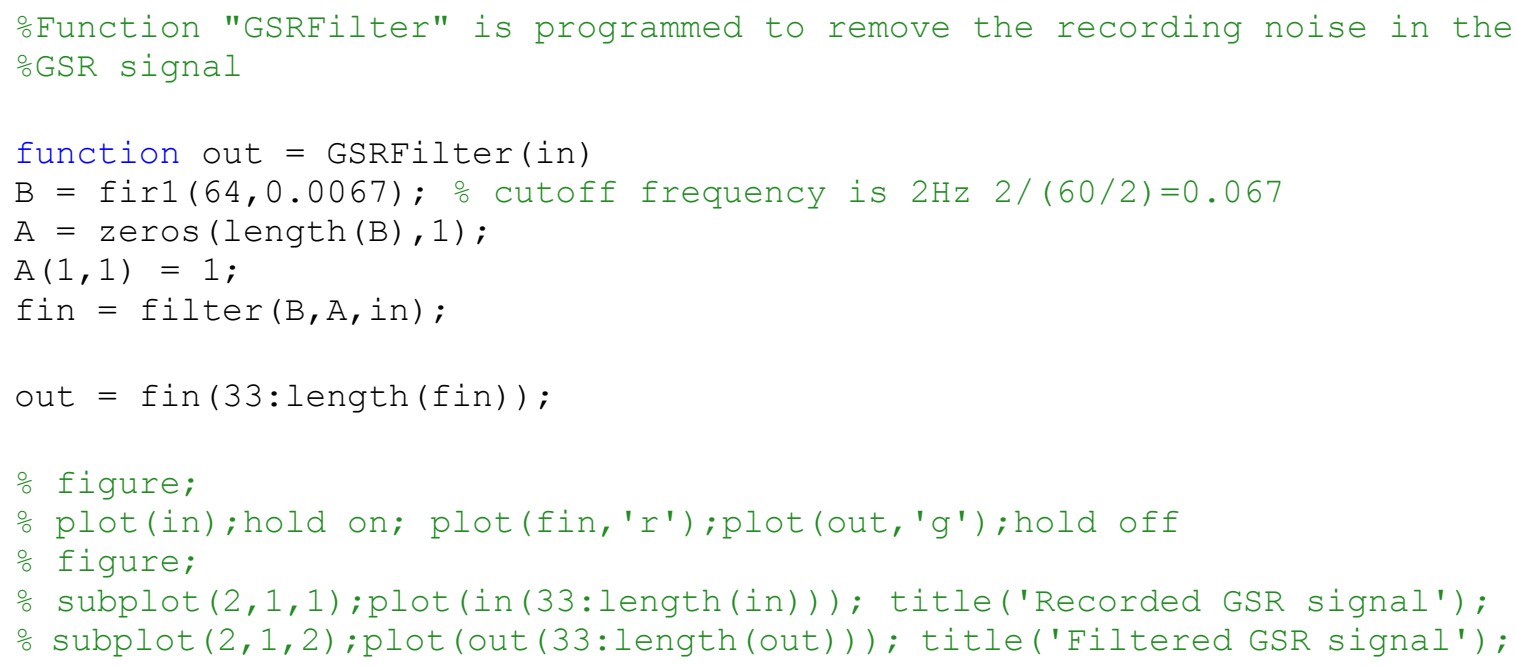

\section{- Function: GSRProc.m}

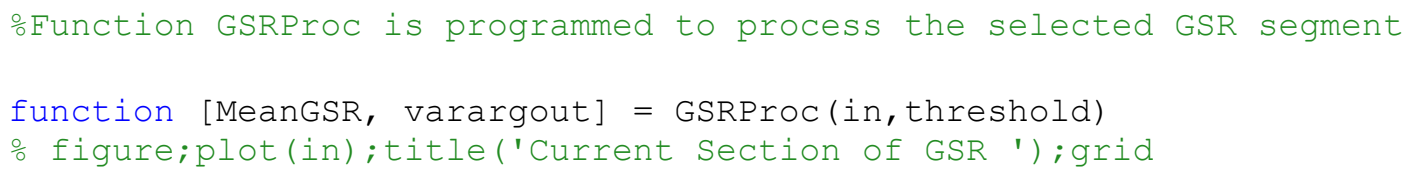




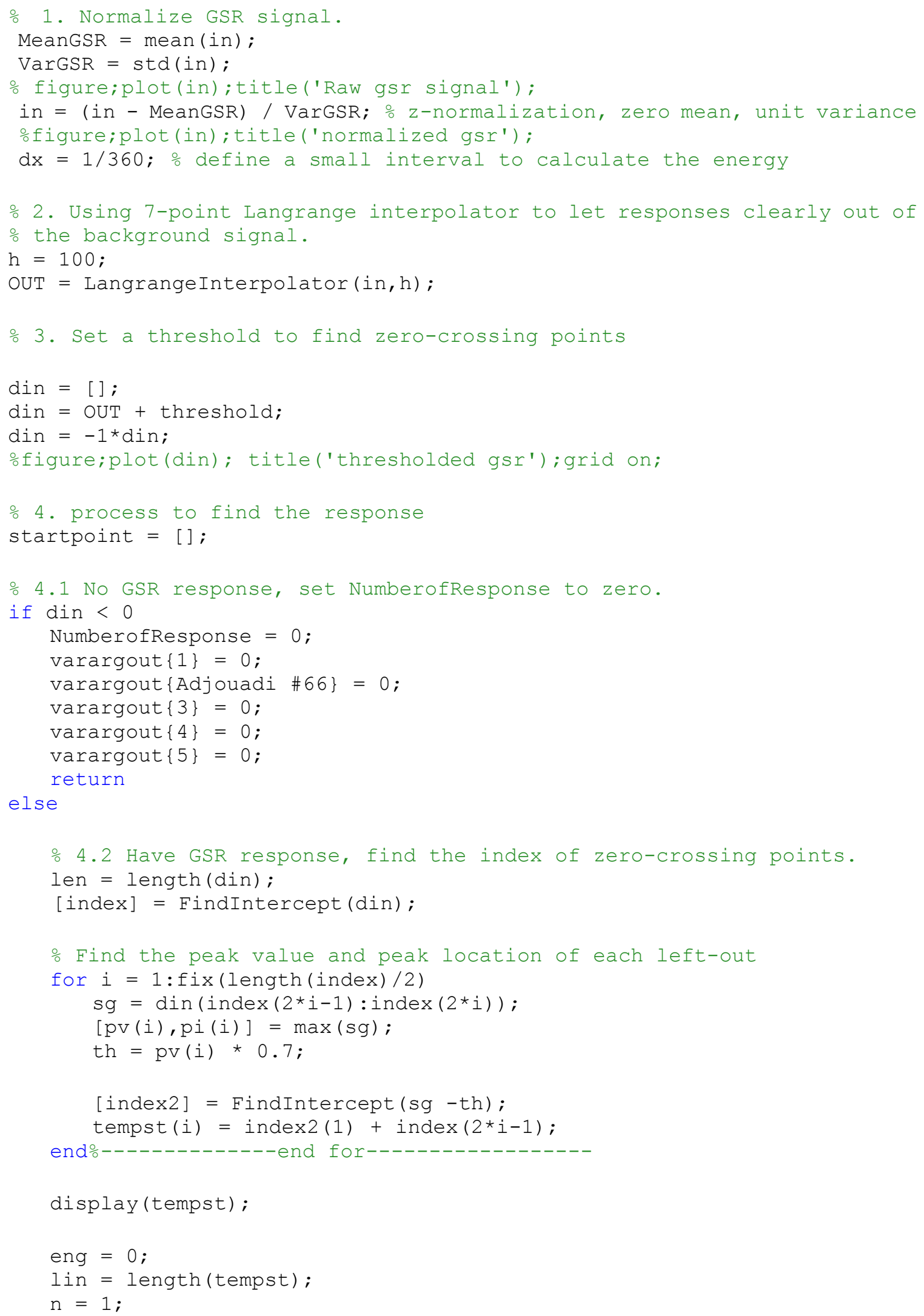




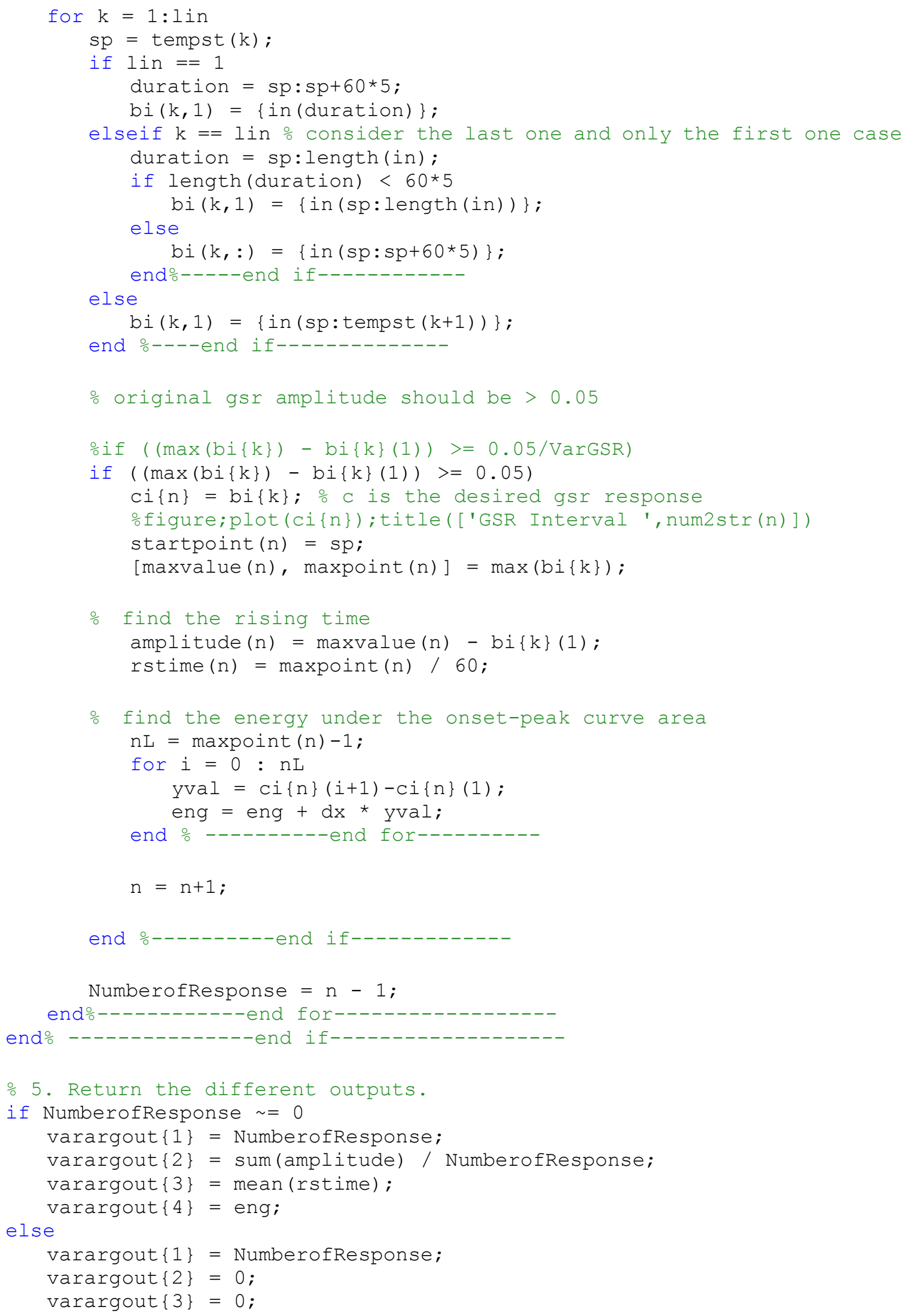


end

varargout $\{4\}=0$;

o display(startpoint);

\section{- Function : LangrangeInterpolator.m}

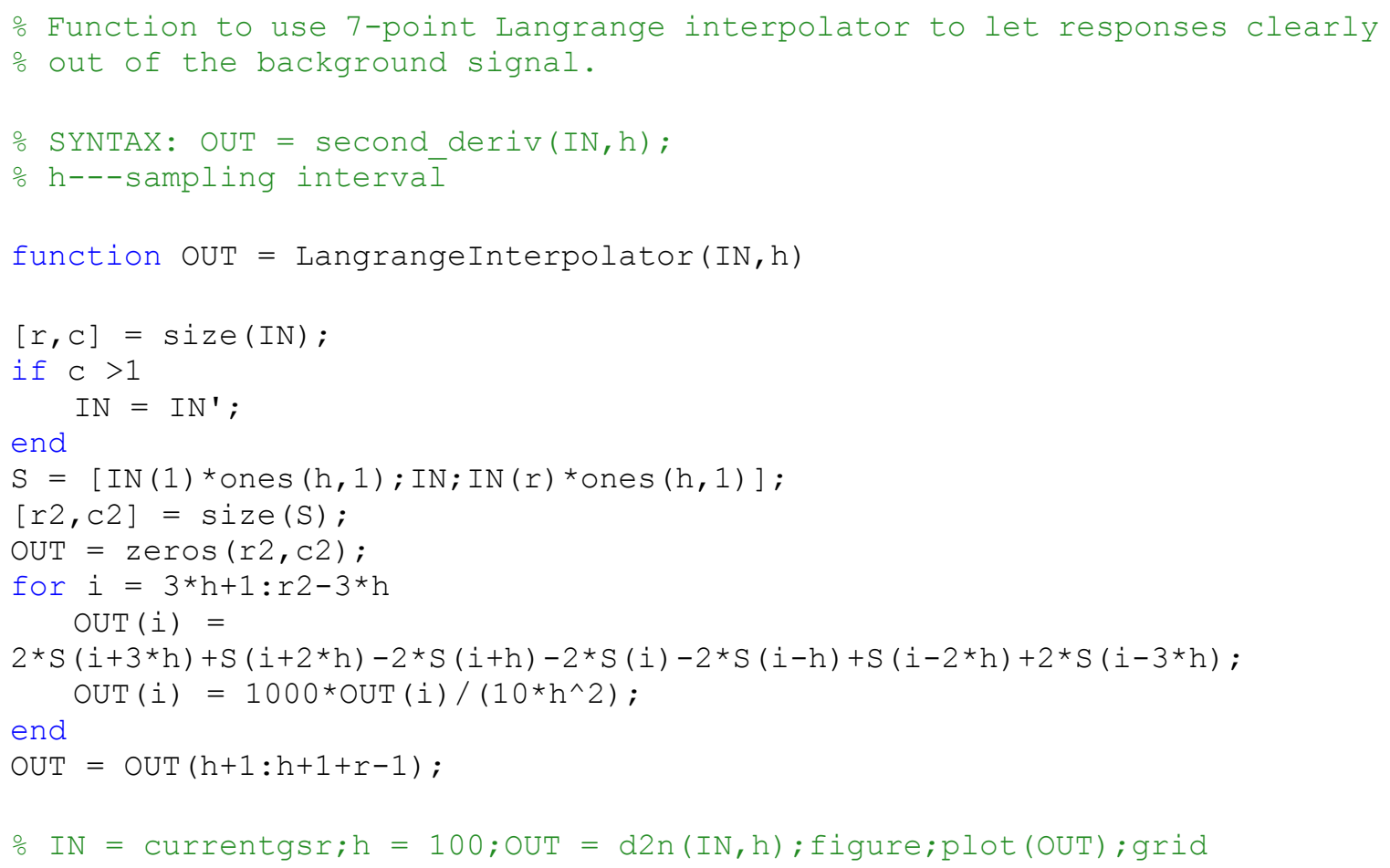

\section{- Function: BVPFeatureExtraction.m}

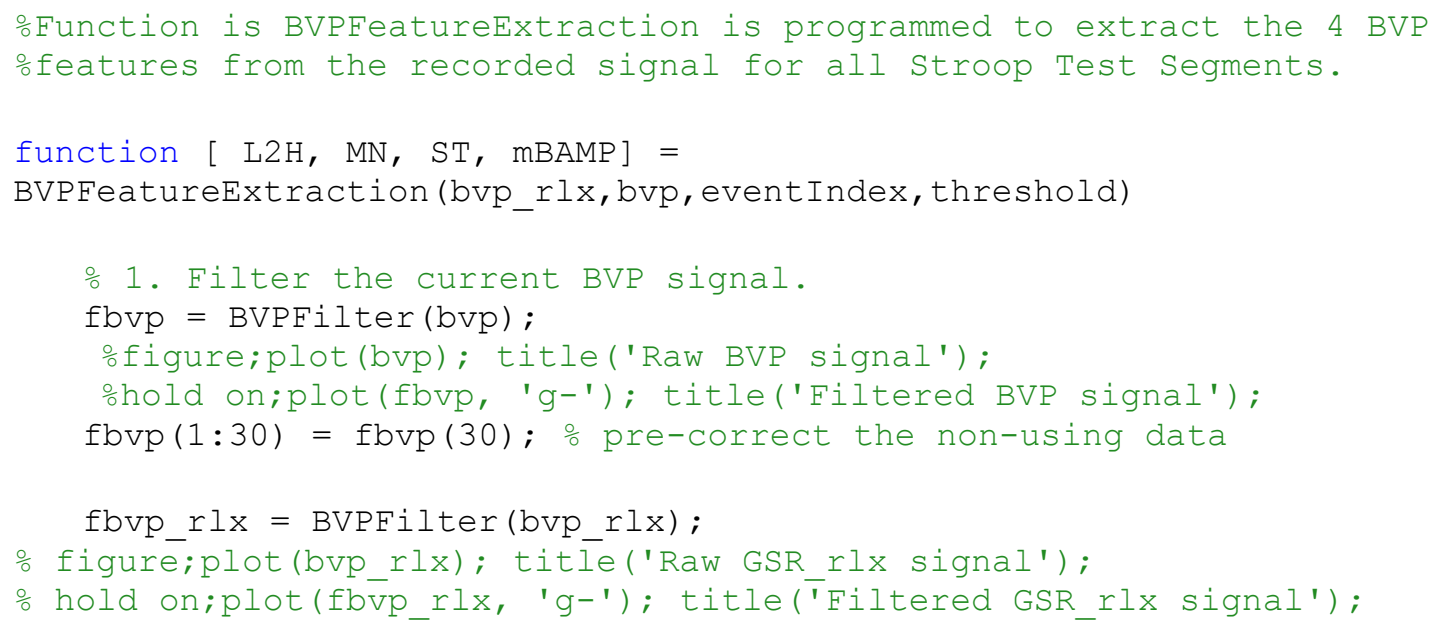




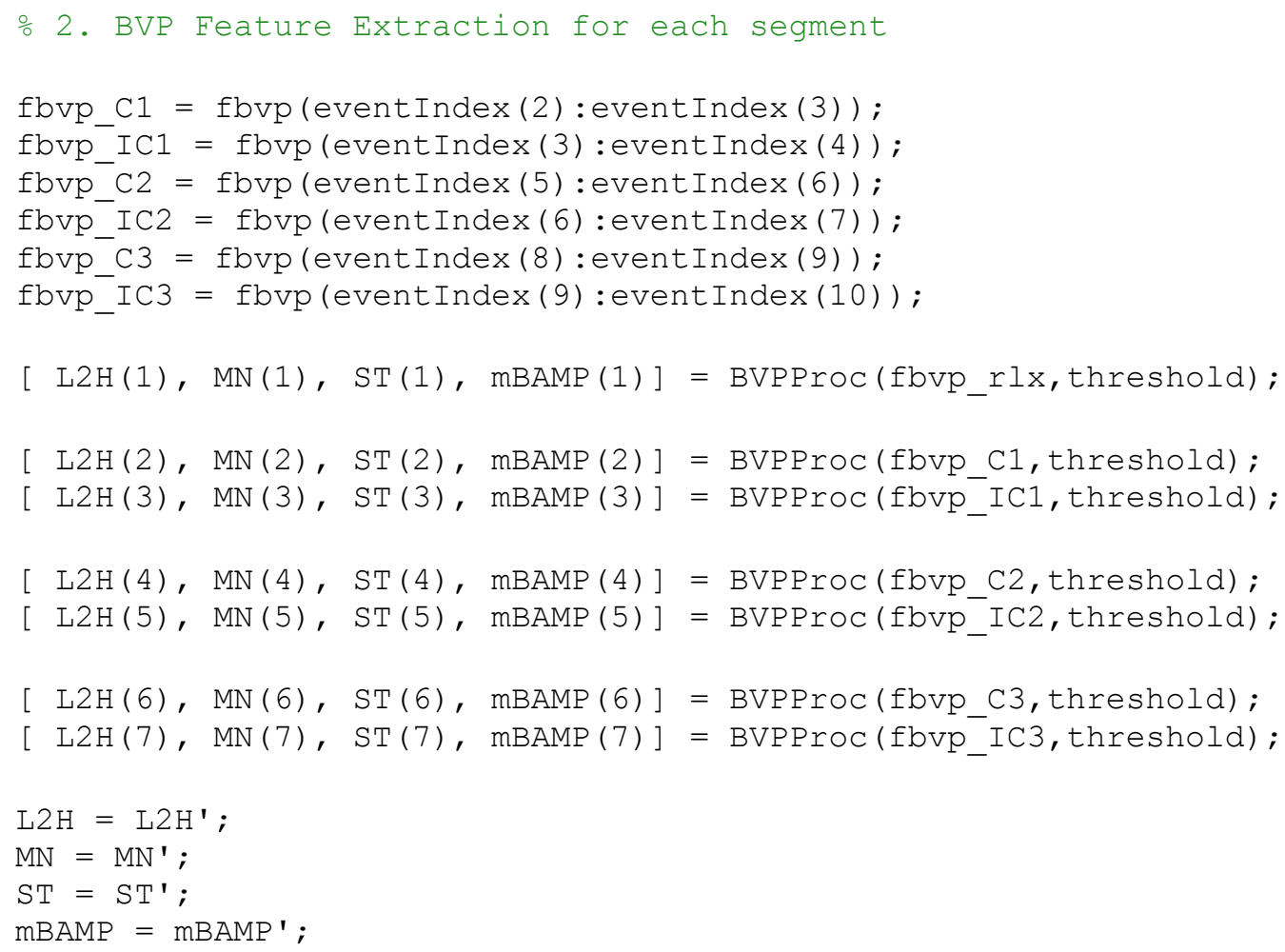

\section{- Function: BVPFilter.m}

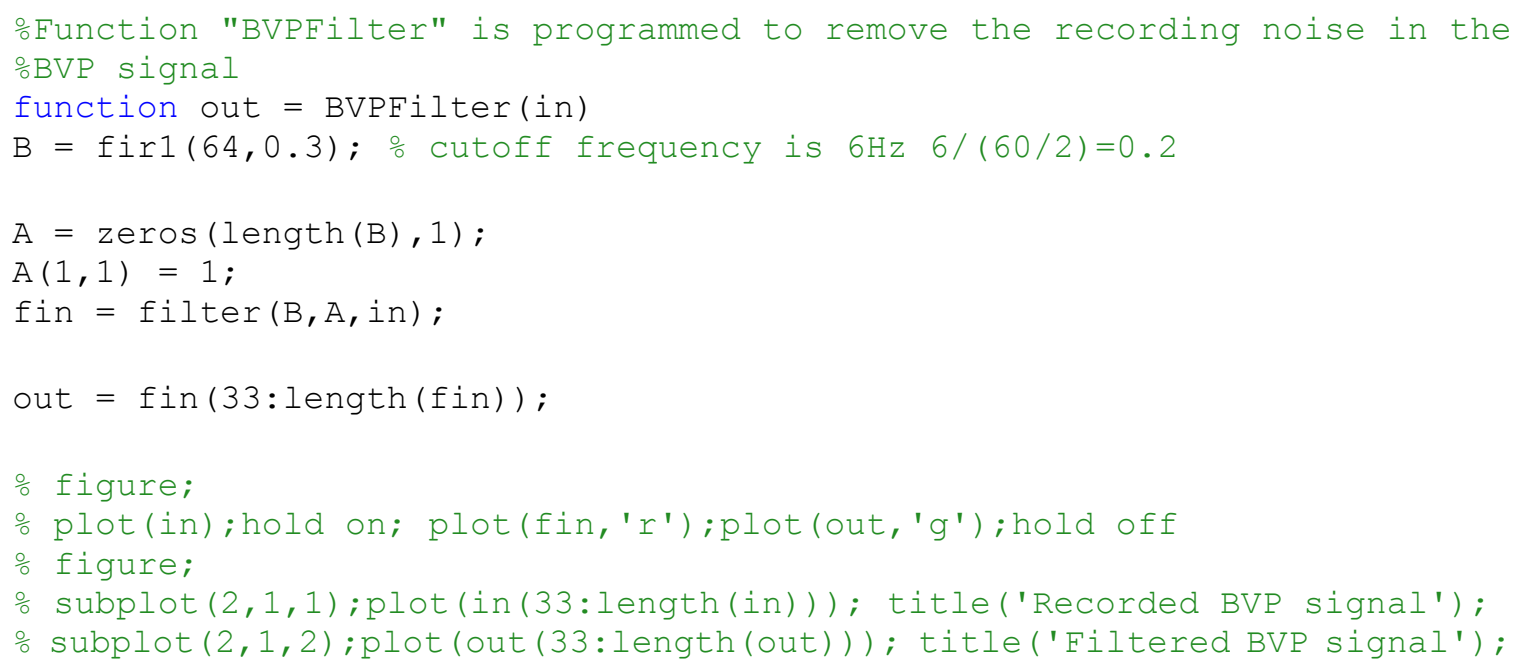




\section{- Function: BVPProc.m}

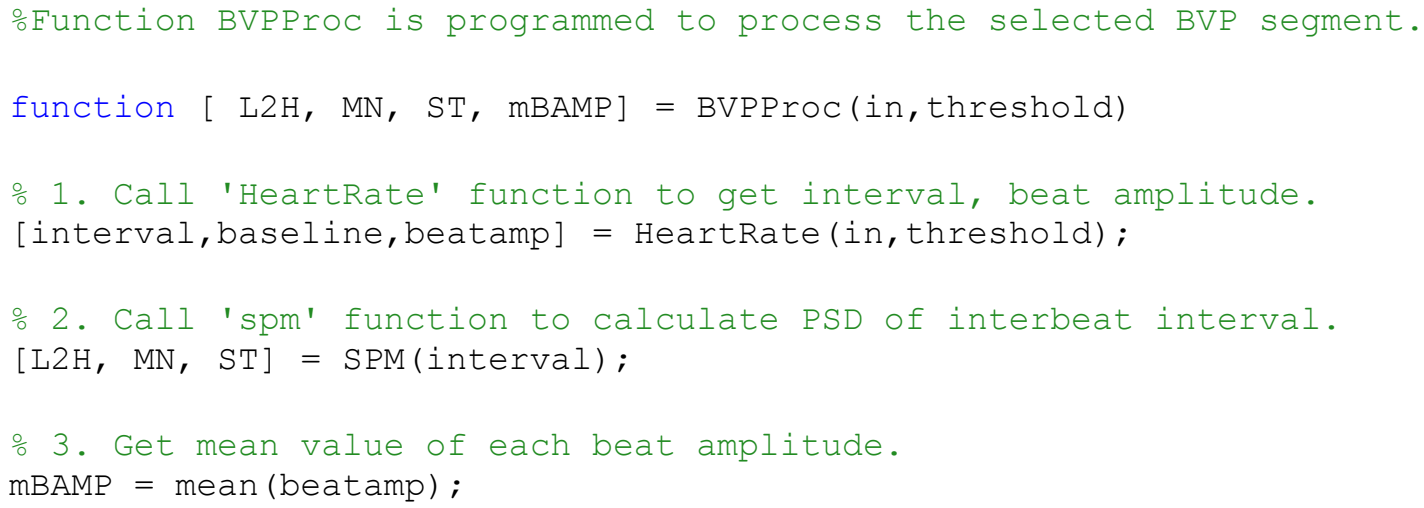

\section{- Function: HeartRate.m}

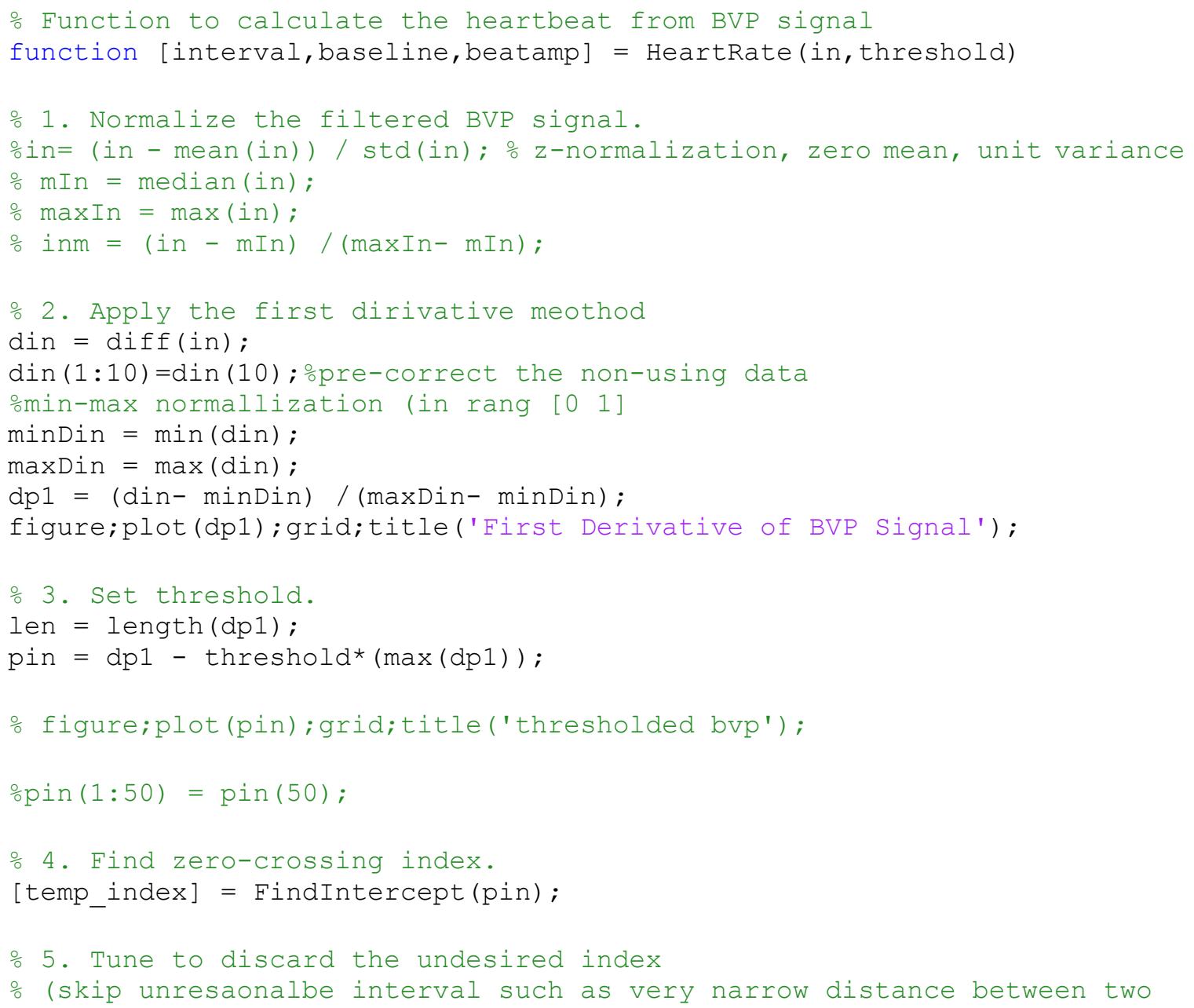




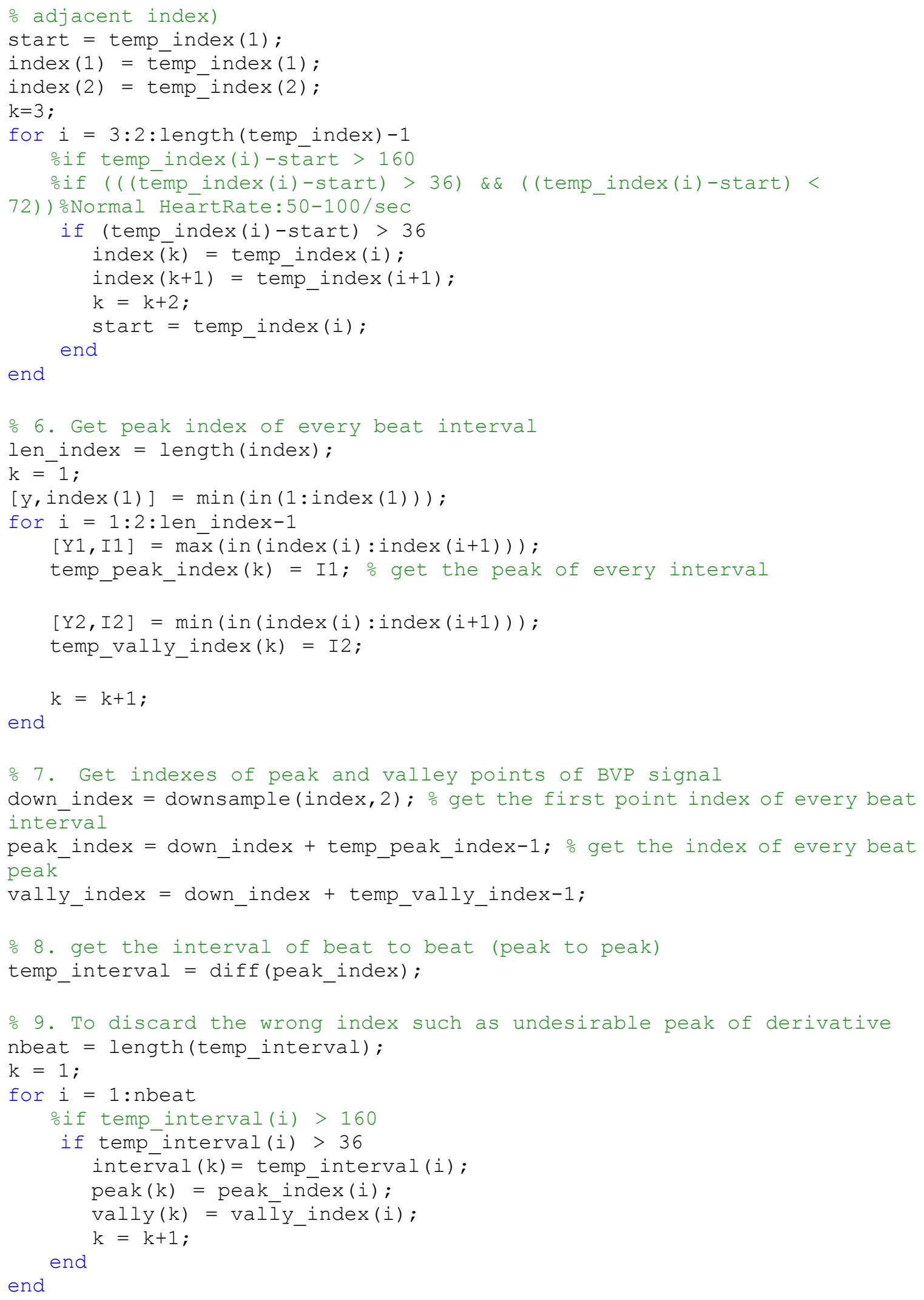




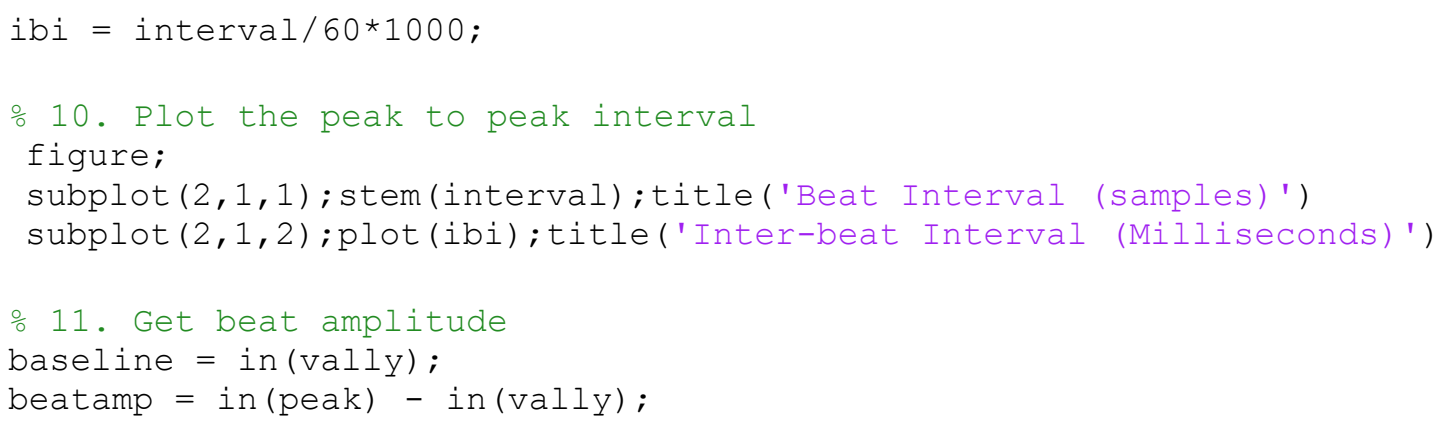

- Function : SPM.m

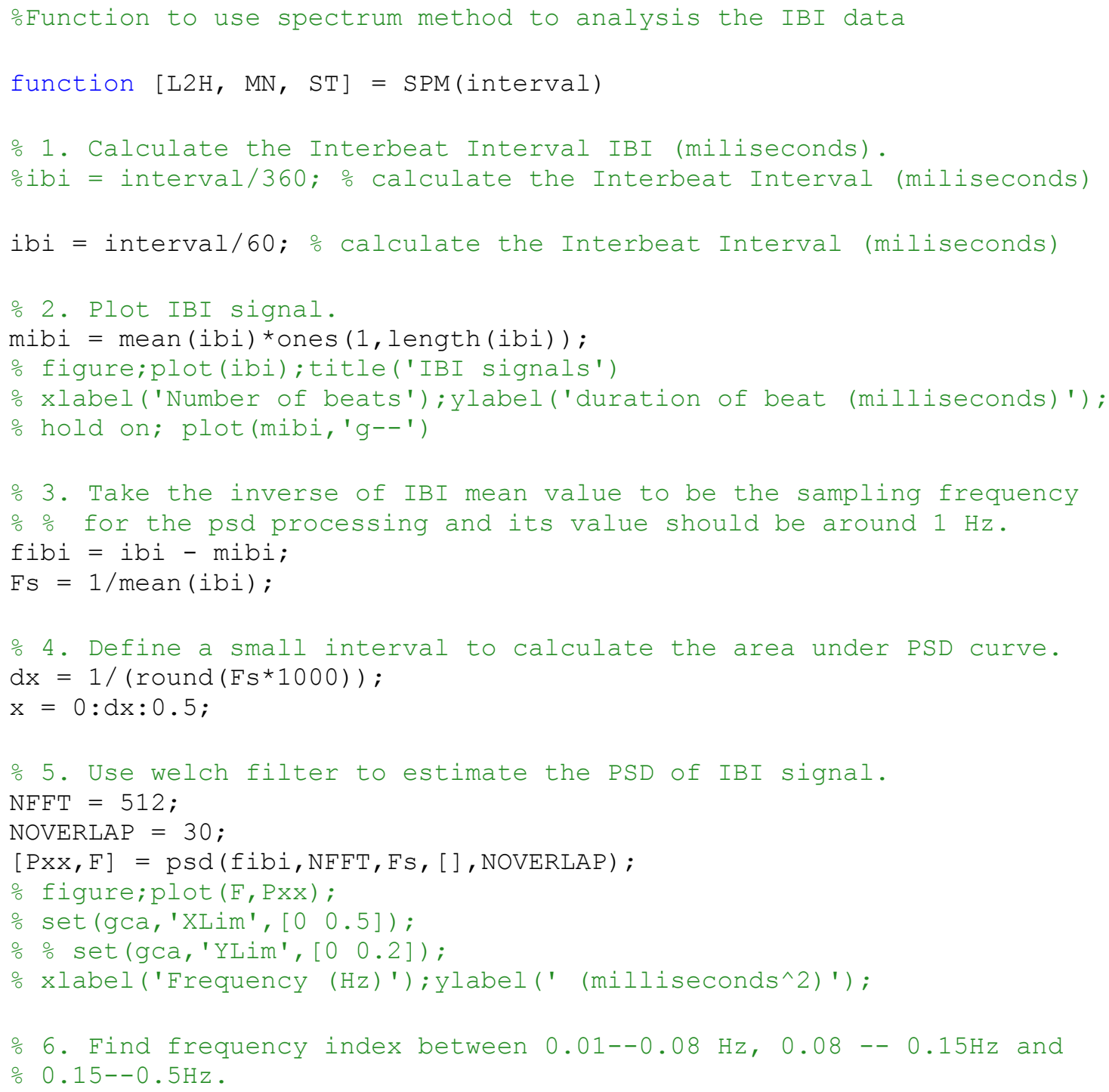




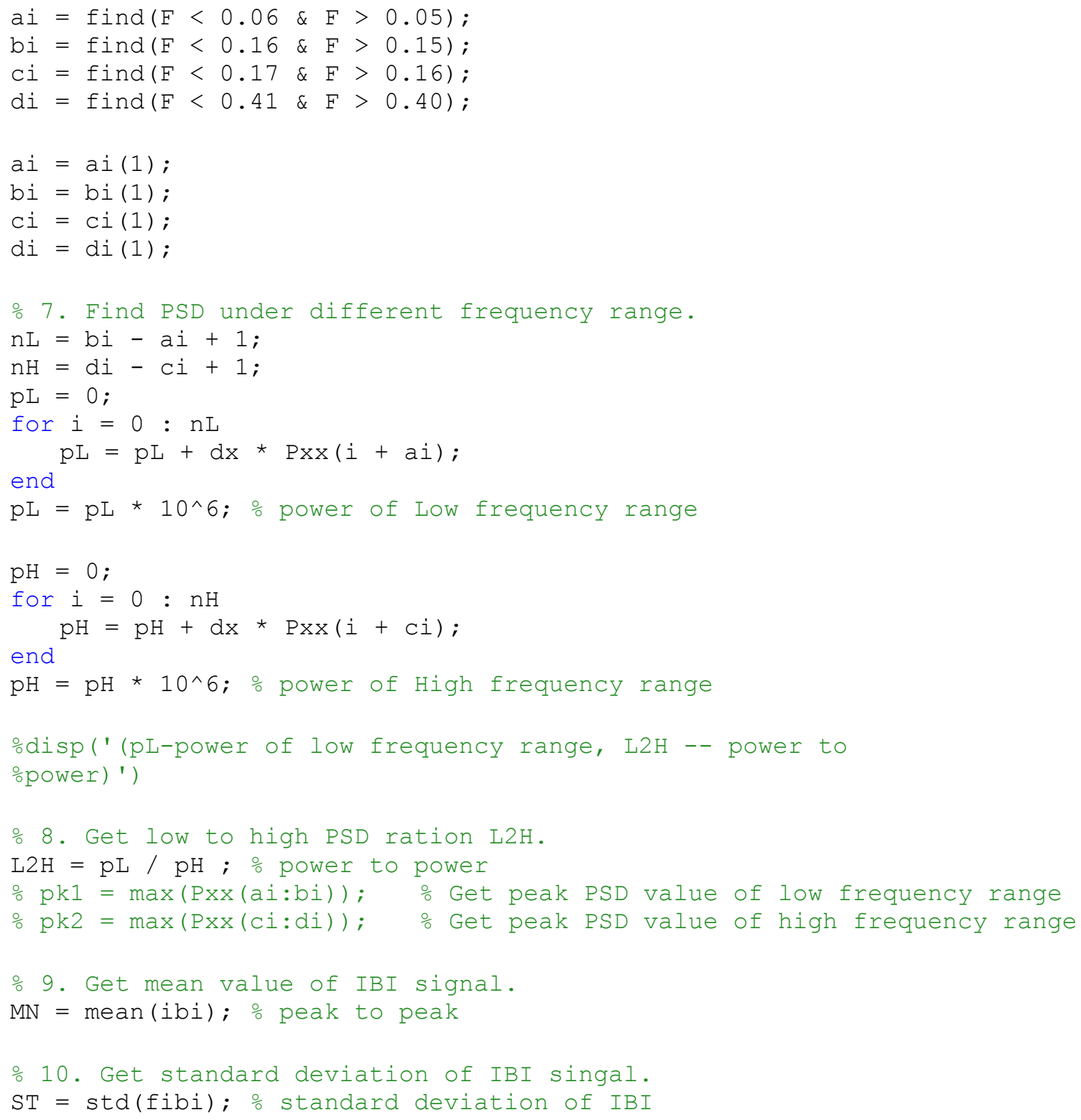


VITA

YING GAO

\section{Education}

Ph.D. Candidate in Electrical Engineering

Florida International University

Dissertation: "A digital Signal Processing Approach for Affective Sensing of a Computer User through Pupil Diameter Monitoring"

Advisor: Armando Barreto

M.S. in Electrical Engineering

Sichuan University

Sep. 2002 - Aug. 2005

Thesis: "Research on a Combination of Time-domain \& Frequency-domain Analysis Method to Power Quality"

Advisor: Honggeng Yang

B.S. in Electrical Engineering

Sichuan University

\section{Honors and Awards}

- 2008-2009

Florida International University Dissertation Year Fellowship Recipient

- 2005-2008

Florida International University Presidential Enhanced Assistantship Recipient

- April 2009

The $2^{\text {nd }}$ place of student poster presentation in Rocky Mountain Bioengineering Symposium.

\section{Publications}

\section{Journals}

[1]. Y. Gao, A. Barreto, and M. Adjouadi, "Detection of Sympathetic Activation through Measurement and Adaptive Processing of the Pupil Diameter for Affective Assessment of Computer Users." American Journal of Biomedical Sciences, vol 1(4), p. 283-294, 2009.

[2]. Y. Gao, A. Barreto, and M. Adjouadi, "Monitoring and Processing of the Pupil Diameter Signal for Affective Assessment of a Computer User." Lecture Notes in Computer Science (LNCS). LNCS 5610, p. 49-58, 2009.

[3]. Y. Gao, A. Barreto, and M. Adjouadi, "Comparative Analysis of Noninvasively Monitored Biosignals for Affective Assessment of a Computer User." International Journal of Medical Implants and Devices, vol. 4, p. 53, 2009. (Abstract)

[4]. Y. Gao, A. Barreto, and M. Adjouadi, "H-infinity Time-varying Adaptive Filtering of Pupil Diameter for Affective Sensing." Biomedical Sciences Instrumentation, vol. 45, pp. 322-327, 2009.

[5]. Y. Gao, A. Barreto, and M. Adjouadi, "Adaptive Interference Cancelling Removal of Pupillary Light Reflex Contributions in Pupil Diameter Variations." International Journal of Medical Implants and Devices, vol. 3, p. 9, 2008. (Abstract) 
[6]. Y. Gao, A. Barreto, K. J. Faller II, and M. Adjouadi, "System Identification for the Contribution of the Light Variations to Pupil Diameter Change." Biomedical Sciences Instrumentation, vol. 44, pp. 389-403, 2008.

[7]. A. Barreto, J. Zhai, N. Rishe, and Y. Gao, "Measurement of Pupil Diameter Variations as a Physiological Indicator of the Affective State in a Computer User," Biomedical Science Instrumentation, vol. 43, pp. 146-151, 2007.

[8]. Y. Gao and H. Yang, "Detection of Power Quality Disturbances Based on Statistical Characteristics of Instantaneous Load," Relay, vol. 32, pp. 11-15, 2004.

\section{Conference Proceedings/Presentation}

[1]. Y. Gao, A. Barreto, and M. Adjouadi, "Comparative Analysis of Noninvasively Monitored Biosignals for Affective Assessment of a Computer User." In The 25th Southern Biomedical Engineering Conference (SBEC '09), International Federation for Medical and Biological Engineering (IFMBE) Proceedings, Miami, Florida USA, 2009, vol 24, pp. 255-260.

*Presented by Ying Gao

[2]. Y. Gao, A. Barreto, and M. Adjouadi, " Comparison of Pupillary Light Reflex System Modeling with $\mathrm{H}^{\infty}$ and LSM Adaptive Algorithms," in Digital Signal Processing Workshop and 5th IEEE Signal Processing Education Workshop, 2009. DSP/SPE 2009. IEEE 13th, Macro Island, Florida USA, 2009, pp. 167-171.

*Presented by Ying Gao

[3]. A. Barreto, Y. Gao, and M. Adjouadi, "Pupil diameter measurements: untapped potential to enhance computer interaction for eye tracker users?" in Proceedings of the 10th international ACM SIGACCESS conference on Computers and accessibility, Halifax, Nova Scotia, Canada, 2008, pp. 269-270.

[4]. Y. Gao, A. Barreto, and M. Adjouadi, "Adaptive Interference Cancelling Removal of Pupillary Light Reflex Contributions in Pupil Diameter Variations," in The 24th Southern Biomedical Engineering Conference (SBEC '08), El Paso, Texas USA, 2008, pp. 51-54.

*Presented by Ying Gao

[5]. Y. Gao, A. Barreto, J. Zhai, and N. Rishe, "Digital Filtering of Pupil Diameter Variations for the Detection of Stress in Computer Users," in The 11th World Multi-Conference on Systemic, Cybernetics and Informatics (WMSCI '07), Orlando, Florida USA, 2007, pp. 30-35.

[6]. Y. Gao and H. Yang, "Locating of Power Quality Disturbances Source with Characteristics of Instantaneous Load," in The 19th International Conference on Electric Power System Automation (EPSA '03), Chengdu, Sichuan, China, 2003, pp. 237-242.

*Presented by Ying Gao

\section{Book Articles}

[1]. Y. Gao, A. Barreto, and M. Adjouadi," Monitoring and Processing of the Pupil Diameter Signal for Affective Assessment of a Computer Use.", has been accepted and will appear in Innovations and Advances in Computer Sciences and Engineering.

[2]. Barreto, J. Zhai, N. Rishe, and Y. Gao, " Significance of Pupil Diameter Measurements for the Assessment of Affective State in Computer Users," in Advances and Innovations in Systems, Computing Sciences and Software Engineering, K. Elleithy, Ed. Netherlands: Springer, 2007, pp. 59-64. 